Universidade de São Paulo

Instituto de Astronomia, Geofísica e Ciências Atmosféricas

Departamento de Astronomia

André Luiz Figueiredo

\title{
O ciclo de vida de discos de decréscimo viscoso em torno de estrelas Be: parâmetros fundamentais de discos da Grande Nuvem de Magalhães
}

\author{
Versão Corrigida. \\ O original encontra-se disponível na Unidade
}

São Paulo 



\title{
O ciclo de vida de discos de decréscimo viscoso em torno de estrelas Be: parâmetros fundamentais de discos da Grande Nuvem de Magalhães
}

\author{
Versão Corrigida. \\ $\mathrm{O}$ original encontra-se disponível na Unidade
}

Dissertação apresentada ao Departamento de Astronomia do Instituto de Astronomia, Geofísica

e Ciências Atmosféricas da Universidade de São Paulo como requisito parcial para a obtenção do título de Mestre em Ciências.

Área de Concentração: Astronomia

Orientador: Prof. Dr. Alex Cavaliéri Carciofi

São Paulo 

ao transcendental; à minha família e amigos 



\section{Agradecimentos}

Gostaria de iniciar os meus agradecimentos para com o grande número de pessoas que tornam possível o funcionamento, e integram também, um ótimo ambiente de trabalho no IAG, especialmente no Departamento de Astronomia. Agradeço à CAPES pelo suporte financeiro e à FAPESP pela infraestrutura proporcionada.

Gostaria de especialmente agradecer ao professor orientador e amigo Alex C. Carciofi e ao Rodrigo G. Vieira por dividirem comigo, além de suas expertises e interesses científicos, momentos de felicidade e sofrimento durante os meus processos de desenvolvimento.

Gostaria de agradecer aos colaboradores J. Bjorkman e Leandro Kerber por dividirem comigo a sua empolgação contagiante pelo trabalho acadêmico.

Muito obrigado Alex e Rímulo pelas revisões e sugestões que me auxiliaram na elaboração desse texto, bem como à Amanda, Geeh e Fernanda pela ajuda para o depósito.

Um agradecimento especial à Igor Soszynski por nos prover os dados que foram essenciais para o desenvolvimento deste trabalho.

Gostaria de agradecer aos professores do Departamento de Astronomia por sempre se dispuseram a conversar com os alunos, especialmente aos que ministraram os cursos que fiz durante o mestrado: Jorge Melendez, Antônio Mário Magalhães (que também é meu relator, e contribuiu muito com seus apontamentos em meus relatórios escritos e apresentados), Marcos Diaz, Vera Jatenco e Jane Hetem (a quem dedico uma gratidão especial, pois através dos seus concelhos optei por me matricular no mestrado). À Professora Arlene Clemesha (FFLCH-USP), que através da sua matéria de cultura árabe, pude ter maior contato com a história da astronomia, fora do eixo ocidental.

Um abraço especial ao nosso grupo de estudos de estrelas Be, os BeACoNs, por inúmeras conversas edificantes e motivadoras: Affonso, Amanda Rubio, Ariane, Arthur Alegre, 
Bruno Mota, Daiane Seriacopi, Daniel Bednarski, Daniel Moser, Fernanda Nogueira, Leandro Rímulo, Lucas Zillner, Marcelo Rubinho, Matheus Genaro, Mohammad Ghoreyshi, Pedro, Rodrigo Vieira, Tajan. Despina, aprendi muito com você, obrigado pelo apoio que você me deu, especialmente ao fim da minha graduação.

Aos colegas do IAG, em especial Andrés, Aura Arévalo, Daniele Aragão, Geeh, Jhon Galarza, Marília Carlos, Johnny, Rafael Campos, Stelinha, Liliane, Natália, Daniele, Julian, Angeles, Fellipy, Renato, Paulo Lago, Lucas Shirbel.

Muitíssimo obrigado ao grupo de terça. As discussões que temos em muito contribuem para que eu me torne uma pessoa melhor.

Gostaria de agradecer ao meu instrutor de Yoga e à Adriana, por me apresentarem a essa prática que me auxilia no equilíbrio e compreensão internos e externos; além de proporcionar um ambiente para discussão de ideias importantes.

Um agradecimento especial aos colegas de USP, por dividirem tantas ideias, vivência e discussões construtivas (e desconstrutivas :P ): André Guedes, Aline \& Bruno, Martha \& Paulo, Stefan, Bruno \& Vivian, Renê, Luiz Henrique e Edson Ponci.

Obrigado ao meus caros amigos por tantos momentos de felicidade e apoio: Yana, Isadora (em especial pelos seus cupcakes motivacionais), Renata, Eder, Priscila \& Lais, Kinga (pela recepção atenciosa em viagem à Polônia), Mauri e Marcia Louzada.

Gostaria de agradecer aos amigos de Lorena, por me tornarem uma pessoa mais completa, motivada e consciente de mim e do ambiente ao meu redor: Renato Amaral \& Marcela Teixeira (em especial), Jean \& Ariadna, Júlio, Ivan, Allan e Paulo.

Por tantos momentos hilários e energizantes, queria agradecer ao pessoal do curso de Libras da AADA, de São José dos Campos, em especial à Silvana, Maria, Amanda, Drika, Danny, Patricia, Rosi e Vitoria.

Acredito que uma das experiências com a qual mais se aprende é poder dividir um projeto comum com outra pessoa. Karina, eu aprendi imensamente com você, no período que ficamos casados, bem como no momento em que decidimos nos separar. Serei eternamente grato por compartilharmos, por anos, a nossa existência.

Gostaria de agradecer a vó Gizelda por tantos momentos de carinho e dificuldades, e pela honra de te acompanhar no seu desencarne; e também ao vô Benedito por todo o seu carinho, amor e despedida especial. Marcos, muito obrigado por me contaminar com sua força, perseverança e entusiasmo pela vida, a convivência com você, por mais de 
uma década e meia, em muito contribuiu para a minha formação. Cássio, muito obrigado por compartilhar a sua visão de mundo, tantos momentos de felicidade e parceria comigo. Através da convivência, aprendi muitíssimo com você! Cida e Osvaldo, muito obrigado por me acolherem como filho. Vocês são pessoas fundamentais na minha vida e me proporcionaram tanto amor, dedicação e atenção, além de inúmeras oportunidades às quais vocês não tiveram acesso.

Gostaria de agradecer imensamente ao pai João e tanto outros amigos espirituais, sem os quais o caminho até aqui não seria possível ou muito mais árduo.

Esta tese/dissertação foi escrita em ATEX com a classe IAGTESE, para teses e dissertações do IAG. 

"Quero viver mais duzentos anos

Quero não ferir meu semelhante

Nem por isso quero me ferir

Vamos precisar de todo mundo

Pra banir do mundo a opressão

Para construir a vida nova Vamos precisar de muito amor A felicidade mora ao lado E quem não é tolo pode ver" Beto Guedes - O sal da terra

"Podemos nos defender de um ataque, mas somos indefesos a um elogio." Sigmund Freud

"A evolução da técnica chegou ao ponto de tornar-nos inermes diante da técnica."

Karl Kraus

"São as nossas escolhas, mais do que as nossas capacidades, que mostram quem realmente somos."

Alvo Dumbledore 



\section{Resumo}

Estrelas Be são estrelas B, na sequência principal, cercadas por um disco circunstelar auto-ejetado cuja evolução é governada pela viscosidade. Essas estrelas são conhecidas por exibirem variabilidade em todos os observáveis e em uma ampla variedade de escalas de tempo, por apresentarem altas taxas de rotação e pulsações não-radiais e por não possuírem campos magnéticos em larga escala. O modelo de decréscimo viscoso (VDD) vem sendo empregado na literatura, de forma satisfatória, para modelar os discos de estrelas individuais e, mais recentemente, amostras de estrelas. Entretanto, ainda é necessário muito trabalho para que os dois parâmetros fundamentais destes discos, a saber, o valor da viscosidade e a taxa de decréscimo viscoso, sejam melhor compreendidos. Curvas de luz de estrelas Be nos oferecem a oportunidade de estudar estes parâmetros, bem como caracterizar a variabilidade destes discos. Utilizamos um procedimento de modelagem (pipeline) que foi recentemente apresentado na literatura, e que foi aplicado para uma amostra de estrelas Be da Pequena Nuvem de Magalhães (Rímulo et al., 2018). A pipeline utiliza curvas de luz sintéticas, que combinam simulações hidrodinâmicas com cálculos de transferência singular para modelar os dados observacionais. Nesta dissertação utilizamos a grade de modelos de Rímulo et al. para estudar uma amostra de curvas de luz da Grande Nuvem de Magalhães observadas pelo levantamento OGLE, cuja janela observacional é de, aproximadamente, 18 anos. Foram analisadas 83 estrelas e 120 eventos de formação e dissipação de disco visando oferecer uma estimativa estatisticamente significativa dos parâmetros estelares (massa, rotação e idade), geométrico (ângulo de inclinação) e do disco (viscosidade, densidade e taxas de perda de massa e momento angular). A taxa de perda de massa e momento angular para os discos mais densos da nossa amostra é da ordem de $10^{-10} M_{\odot}$ ano $^{-1} \mathrm{e} \sim 10^{37} \mathrm{~g} \mathrm{~cm}^{2} \mathrm{~s}^{-1}$. Os valores desses parâmetros estão abaixo do previsto 
por modelos de evolução estelar em alta rotação, resultado semelhante ao estudo anterior feito para a SMC. Em nossa análise, exploramos a possibilidade da viscosidade possuir valores diferentes durante as fases de construção e dissipação do disco. Os valores médios encontrados para a fase de construção $\left(\alpha_{\mathrm{bu}}=0,59_{-0,49}^{+0,58}\right)$ são da ordem de alguns décimos, resultado que é consistente com trabalhos recentes na literatura, além de ser compatível com estimativas desse parâmetros para novas anãs. Entretanto, nossos resultados para a viscosidade média da fase dissipativa é 10 vezes menor $\left(\alpha_{\mathrm{d}}=0,06_{-0,05}^{+0,40}\right)$. Por um lado, há uma semelhança qualitativa entre o estudo para a SMC e nosso resultado, pois em ambos os casos $\alpha_{\mathrm{bu}}>\alpha_{\mathrm{d}}$. Por outro lado, o valor médio de $\alpha_{\mathrm{d}}$ para a LMC é muito menor que para a SMC, um resultado consistente com o fato de termos observado em nossa amostra eventos de dissipação de disco muito longos. Os resultados aqui apresentados devem ser vistos ainda com cautela, pois ainda se fazem necessários o cômputo de uma grade hidrodinâmica apropriada à LMC e uma melhor estimativa do avermelhamento interestelar. Uma vez concluídos, os resultados para a rica amostra aqui levantada contribuirão para o estudo dos processos de perda de massa em estrelas Be e da viscosidade associada a estes discos. 


\section{Abstract}

Classical Be stars are rapidly rotating B stars with emission lines, which originate from a self-ejected ionized gaseous disk whose evolution is governed by viscosity. These stars are known to be fast-rotators and non-radial pulsators, and they show variability in all observables in a wide range of time scales. They do not present evidence of large scale magnetic fields. The viscous decretion disk (VDD) theory has been satisfactory applied to model the disks of individual Be stars and, recently, to samples of stars. However, further work is still necessary to have a better understanding of two fundamental disks' parameters: viscosity and viscous decretion rate. Light curves of Be stars offer an opportunity to study these parameters, and characterize the disks' variability. We use a pipeline, recently introduced in the literature, that was used to study a sample of Be stars of the Small Magellanic Cloud (Rímulo et al., 2018). The pipeline uses synthetic light curves, computed from hydrodynamics and radiative simulations, to model the observational data. In this work we use the grid models of Rímulo et al. (2018) to study a sample of light curves from the Large Magellanic Cloud (LMC) observed by the OGLE survey for nearly 18 years. We analyzed 83 stars and 120 disk formation and dissipation events aiming at offering an statistically significant evaluation of stellar (mass, rotation rate, age), geometric (inclination angle) and disk (density, viscosity, mass and angular loss rate) parameters. The mass and angular loss rate of densest disks of our sample are of the order of $\sim 10^{-10} M_{\odot}$ ano $^{-1}$ and $\sim 10^{37} \mathrm{~g} \mathrm{~cm}^{2} \mathrm{~s}^{-1}$. These values are lower than what is predicted by models of fast-rotating stars, in agreement to what was obtained for the SMC. In our analysis, we allow the viscosity parameter to have different values at build-up and dissipation phases of the disk. The median values found for the viscosity during disk build up $\left(\alpha_{\mathrm{bu}}=0,59_{-0,49}^{+0,58}\right)$ are typically of a few tenths, which is consistent with recent works in the literature, and in agreement 
with estimates from dwarf novae. However, our results for the median value of viscosity during dissipation is 10 times lower $\left(\alpha_{\mathrm{d}}=0,06_{-0,05}^{+0,40}\right)$. While there is a qualitative similarity between our study and the results for the SMC, since in both cases $\alpha_{\mathrm{bu}}>\alpha_{\mathrm{d}}$, the median value of $\alpha_{\mathrm{d}}$ for the LMC is much lower than the SMC value, which is consistent with the fact that our sample contains very long dissipative disk events. The results presented in this work should be seen with caution, since a model grid taylored for the LMC must still be computed, and a better determination of the interstellar reddening is still need. Once concluded, the results of the large sample presented here will contribute to the study of the mass loss processes in Be stars and the associated viscosity in their disks. 


\section{Sumário}

1. Introdução . . . . . . . . . . . . . . . . . . . . . . . . . 25

1.1 Contexto astrofísico . . . . . . . . . . . . . . . . . . . 25

1.2 Estrelas de alta massa f . . . . . . . . . . . . . . . . . . . . . . 28

1.2.1 Estrelas quentes e a rotação estelar . . . . . . . . . . . . . . . . . 29

1.3 Estrelas $\mathrm{Be} \ldots \ldots \ldots \ldots \ldots \ldots \ldots$

1.3.1 Estrelas quentes peculiares . . . . . . . . . . . . . 31

1.3.2 Contexto Histórico . . . . . . . . . . . . . . . . . . . . . . . . . . . 32

1.3.3 Observáveis de estrelas Be . . . . . . . . . . . . . . . . . 35

1.4 Modelo de decréscimo viscoso . . . . . . . . . . . . . . . . . . . . 41

1.5 Relevância astrofísica de estrelas Be . . . . . . . . . . . . . . . . . . 42

1.6 Objetivos e organização do trabalho . . . . . . . . . . . . . . . . . 43

2. Discos dinâmicos de decréscimo viscoso . . . . . . . . . . . . . . . . . . . 45

2.1 Revisão histórica . . . . . . . . . . . . . . . . . . . . 45

2.1.1 O efeito de reservatório de massa . . . . . . . . . . . . . 47

2.1.2 Bumps e Dips . . . . . . . . . . . . . . . . . . . . 49

2.1.3 Modelagem de uma amostra de estrelas Be: uma breve revisão . . . 50

2.2 Principais considerações teóricas . . . . . . . . . . . . . . . 51

2.2.1 Injeção e perda de massa no disco . . . . . . . . . . . . . . . . . 54

2.3 Uma grade de curvas de luz sintéticas . . . . . . . . . . . . . . 56

2.3.1 O código SINGLEBE: simulações dinâmica de discos . . . . . . . . 57

2.3.2 Cálculo do transporte de energia com HDUST . . . . . . . . . . 58

2.4 Modelagem de curvas de luz por inferência Bayesiana . . . . . . . . . . . . 63 
3. Curvas de luz de estrelas Be da LMC . . . . . . . . . . . . . . . . . . . . . . . . 71

3.1 Levantamentos fotométricos . . . . . . . . . . . . . . . . . . . 71

3.2 Levantamentos existentes de curvas de luz de estrelas Be das Nuvens de Magalhães . . . . . . . . . . . . . . . . . . . . . . 72

3.3 Curvas de luz de candidatas a estrelas Be . . . . . . . . . . . . . 74

3.4 Seleção de bumps para a Grande Nuvem de Magalhães . . . . . . . . . . . 77

3.4 Propriedades globais . . . . . . . . . . . . . . . 79

3.4.2 Bumps extremamente longos . . . . . . . . . . . . . . . 82

3.5 Descrição da natureza da amostra de curvas . . . . . . . . . . . . . . . 83

3.5.1 Fenômenos periódicos e quase-periódicos . . . . . . . . . . . . . 84

3.6 Morfologia de curvas de luz . . . . . . . . . . . . . . 86

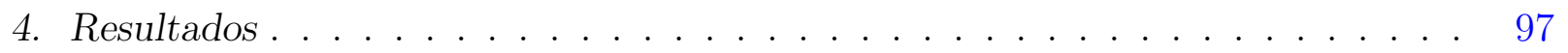

4.1 A influência da rotação nas curvas de luz . . . . . . . . . . . . . . . . . . 97

4.2 Modelagem das estrelas LMC 162.7.108765 e LMC 127.1.38725 . . . . . . . 99

4.3 Resultados para toda amostra de estrela . . . . . . . . . . . . . . 103

4.3.1 Distribuição de Massa . . . . . . . . . . . . . . . . . . . . . . . . . 104

4.3.2 Densidade superficial assintótica . . . . . . . . . . . 105

4.3.3 Duração da fase de construção dos discos . . . . . . . . . . . . . 106

4.3.4 Viscosidade . . . . . . . . . . . . . . . . . 107

4.3.5 Taxas de perda de massa e momento angular . . . . . . . . . . . . . 109

5. Conclusões . . . . . . . . . . . . . . . . . . . . . . . . . . . 121

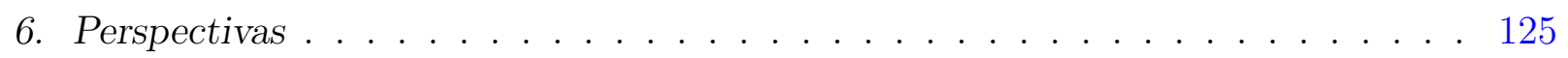

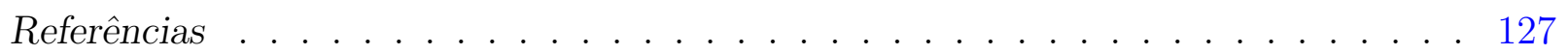




\section{Lista de Figuras}

1.1 Diagrama HR representativo das estrelas da vizinhança solar . . . . . . 26

1.2 Efeito da rotação estelar para estrelas de diferentes massas nos respectivos caminhos evolutivos no diagrama HR . . . . . . . . . . . . . . . . . 30

1.3 Figura esquemática de como o ângulo de inclinação altera os perfis de linhas espectrais . . . . . . . . . . . . . . . . . 34

1.4 Modelagem da SED da estrela Be $\delta$ Scorpii para a fase ativa (com disco) . 36

1.5 Representação esquemática de diferentes regimes ópticos que moldam a emissão do disco . . . . . . . . . . . . . . . . . . 37

1.6 Locais de formação no disco para a emissão do contínuo para várias bandas

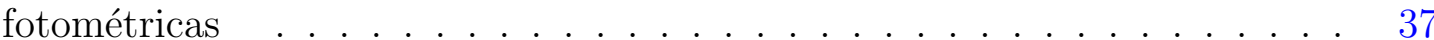

1.7 Variações $V / R$ para diferentes fases do ciclo para as linhas de Balmer su-

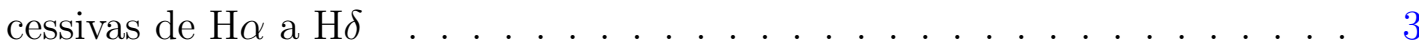

1.8 Figura ilustrativa da formação da polarização divido a um envoltório circunstelar .......................... . . 40

2.1 Modelagens dinâmica de curvas de luz da estrela Be $28 \mathrm{CMa}$. . . . . . . . 46

2.2 Comparação entre dois eventos de formação e dissipação de discos, com durações de 3 e 10 anos . . . . . . . . . . . . . . . . . . 48

2.3 Previsão do efeito da inclinação nas curvas fotométricas de uma estrela Be durante evento de formação e dissipação de disco . . . . . . . . . . . . . . 50

2.4 Dependência das velocidades radial, azimutal e da taxa de perda de momento angular para um modelo de disco isotérmico para diferentes valores de viscosidade . . . . . . . . . . . . . . . . . . . 53

2.5 Média espectral e polarimétrica para a estrela $\zeta$ Tauri . . . . . . . . . . 58 
2.6 Impacto da evolução estelar nos parâmetros da estrela . . . . . . . . . . 59

2.7 Previsão do aumento do tempo de permanência da estrela na Sequência Principal devido ao efeito de rotação . . . . . . . . . . . . . . . . 60

2.8 Resultado da modelagem da curva de luz de uma estrela Be da Pequena Nuvem de Magalhães pela pipeline desenvolvida por Rímulo et al. (2018) 68

3.1 Curvas de luz (banda $V)$ e de cores $(V-R)$ que exemplificam as cinco morfologias identificadas por Keller et al. (2002) para estrelas Be . . . . . 72

3.2 Curvas de luz observadas pelo levantamento OGLE-II para estrelas candidatas a Be para morfologia de tipo $1 \ldots \ldots$. . . . . . . . . . 73

3.3 Idem à Fig. 3.2 para morfologias de tipo 1, 2, 3 e $4 \ldots \ldots$. . . . . . 75

3.4 Diagrama de cor-magnitude de estrelas estrelas selecionadas para este estudo juntamente com as estrelas sintéticas da grade de modelos fotosférica . . . 78

3.5 Histograma para amplitude e tempos de construção, dissipação e duração total para a amostra completa de eventos de disco . . . . . . . . . . . 80

3.6 Tempo de dissipação contra tempo de construção da amostra completa de eventos de disco . . . . . . . . . . . . . . . . . . . . 81

3.7 Curva de luz da estrela LMC 111.6.66352 com o maior número de eventos de disco observado . . . . . . . . . . . . . . . . . . . . . 82

3.8 Idem à Fig. 3.7 para duas curvas de luz que apresentam bumps extremamente longos . . . . . . . . . . . . . . . . . . 83

3.9 Exemplares de características morfológicas da amostra completa de curvas

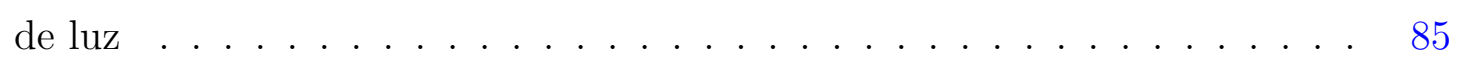

3.10 Periodogramas para estrelas quase periódicas e periódicas . . . . . . . 87

4.1 Impacto da idade e taxa de rotação na amplitude do bump na banda $I$, para estrelas de 7 e $15 M_{\odot} \ldots \ldots \ldots \ldots$. . . . . . . . . . . . . . . . 98

4.2 Idem à Fig. 4.1 para a banda fotométrica $V \ldots \ldots$. . . . . . . . . . . . . 99

4.3 Resultado da modelagem da curva de luz da estrela Be LMC 127.1.38725 101

4.4 Idem à figura 2.8 para a estrela LMC 162.7 .108765 . . . . . . . . . . . . . 102

4.5 Histograma das probabilidades para o parâmetro $M$ e a Função de Massa Inicial (IMF) ponderada pela razão entre estrela Be e B . . . . . . . . . 104 
4.6 Gráfico de caixa para a soma das distribuições posteriores para $\Sigma_{0}$ (acima) e $t_{2}-t_{1}$ (abaixo) para a somatória das distribuições posteriores para os bumps da amostra, divididos em oito intervalos iguais de massa . . . . . . . . 105

4.7 Distribuição dos parâmetros $M$ e $\Sigma_{0}$ da amostra de estrelas Be da Grande Nuvem de Magalhães (LMC), juntamente com os resultados para estrelas da Via Láctea, bem como o valor esperado para um modelo de disco ablativo 106

4.8 Idem à Fig. 4.6 para os parâmetros $\alpha_{\text {bu }}$ (acima) e $\alpha_{\mathrm{d}}$ (abaixo) . . . . . . . 107

4.9 Distribuição das taxas de perda de massa e momento angular para a amostra de estrelas Be da LMC e da Galáxia, em conjunto com estimativas de modelos de evolução estelar para estrelas da LMC e da Pequena Nuvem de

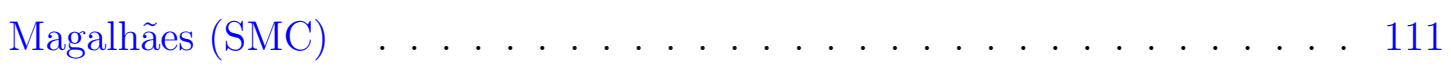





\section{Lista de Tabelas}

1.1 Classificação estelar em tipos espectrais . . . . . . . . . . . . . . . 27

1.2 Classificação estelar por luminosidade . . . . . . . . . . . . . . . . . . . . 28

1.3 Propriedades espectrais selecionadas de estrelas quentes peculiares _ . . . 33

2.1 Parâmetros da grade de modelos de bumps . . . . . . . . . . . . . 61

2.2 Parâmetros estelares dos modelos da Tab. 2.1 . . . . . . . . . . . . . . . . 62

2.3 Parâmetros da grade de modelos fotosféricos . . . . . . . . . . . . . . . . 62

3.1 Número de estrelas que exibem mais de um bump . . . . . . . . . . . . . . 81

3.2 Estrelas Be e os respectivos bumps selecionados para este estudo . . . . . . 89

4.1 Parâmetros para a grade de modelos estacionários . . . . . . . . . . . . 98

4.2 Resultados da pipeline para cada estrela e bump da amostra . . . . . . . . 112 



\section{Lista de Acrônimos}

AGNs Núcleos Ativos de Galáxias.

CQE Central Quasi-Emission.

GRB Surtos de Raios Gama.

IMF Função de Massa Inicial.

$L B V$ Luminosas Azuis variáveis.

LMC Grande Nuvem de Magalhães.

NLTE Equílibrio Termodinâmico Não Local.

SMC Pequena Nuvem de Magalhães.

SPH Smoothed-Particle Hydrodynamics.

UV Ultra Violeta.

VDD Disco de decréscimo viscoso.

WR Wolf-Rayet. 

Capítulo 1

\section{Introdução}

\subsection{Contexto astrofísico}

Estrelas são as principais fontes de informação sobre o universo graças à radiação eletromagnética produzida por esses astros. Contudo, elas não são iguais. Isso fica claro quando olhamos para o céu noturno estrelado e notamos que há estrelas de diferentes brilhos e cores. Essa variedade é resultado da combinação de características intrínsecas desses objetos e de suas distâncias até nós.

A natureza das estrelas começou a ser melhor compreendida com os trabalhos pioneiros e independentes de Ejnar Hertzsprung, em 1911, e por Henry Norris Russell, em 1913. Ao organizarem em um diagrama a luminosidade (ou magnitude absoluta) das estrelas e suas respectivas cores (ou temperaturas superficiais) notaram que os astros não estão distribuídos aleatoriamente. Posteriormente esse diagrama ficou conhecido como diagrama de Hertzsprung-Russell, ou apenas diagrama HR; veja Fig. 1.1. A maioria das estrelas se encontra ao longo de uma faixa estreita diagonal, chamada de "sequência principal" (SP), onde as estrelas mais brilhantes são também as mais azuis. As estrelas que se localizam na SP possuem massas entre 0,08 e 100 massas solares $\left(M_{\odot}\right)$ aproximadamente, e queimam hidrogênio em seus núcleos. A sequência principal corresponde à fase mais longa da existência de uma estrela, o que explica o fato da maioria das estrelas se encontrarem nessa região. Ao fim da queima de todo o hidrogênio contido no núcleo, a estrela deixa a sequência principal para se tornar uma gigante ou uma supergigante, no caso das estrelas mais massivas. Astros com massa próxima à solar evoluem para uma anã branca, ao passo que estrelas de maior massa podem se tornar supernovas, estrelas de nêutrons e buracos negros. 


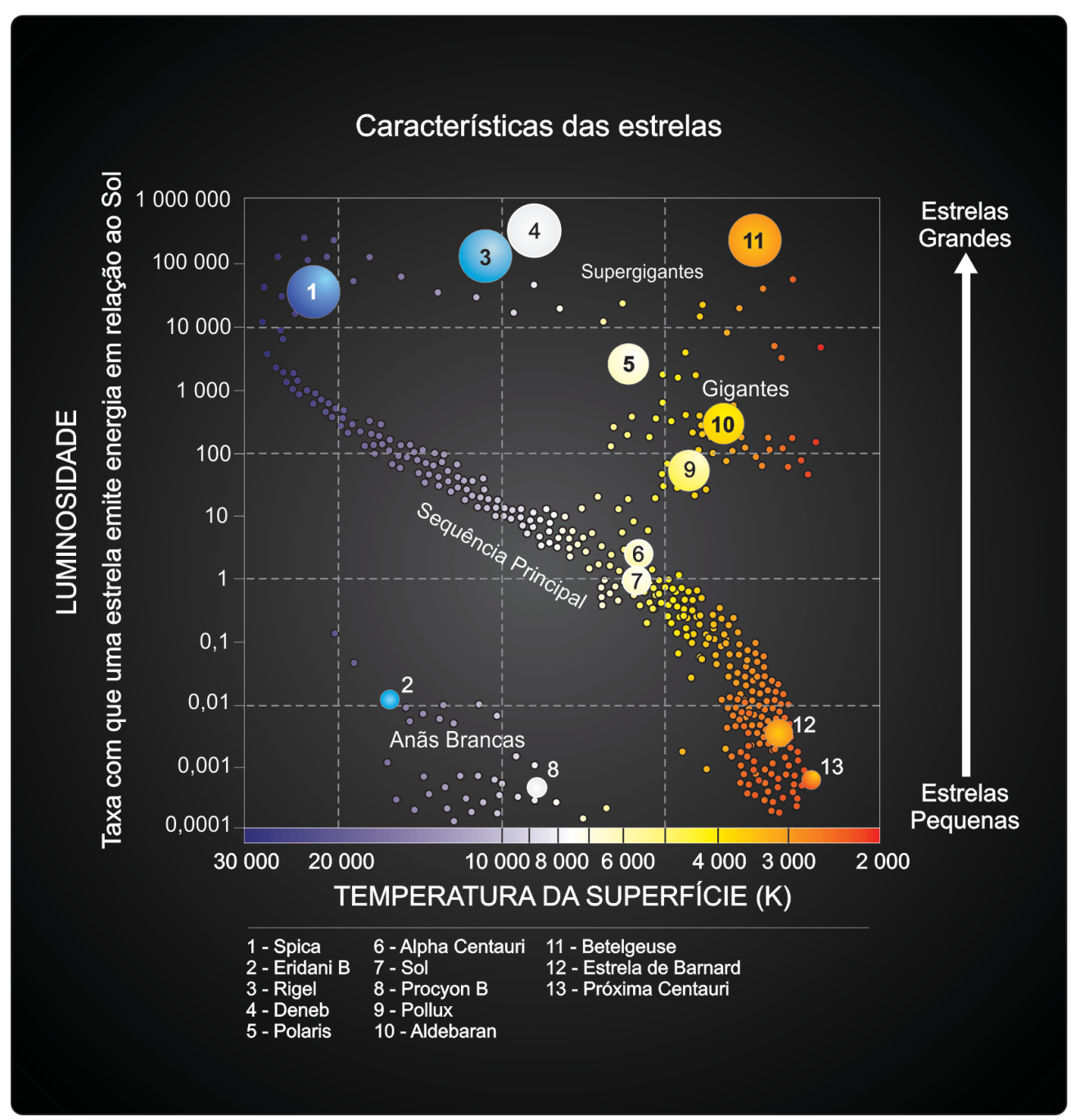

Figura 1.1: Diagrama HR esquemático representativo das estrelas da vizinhança solar: a luminosidade das estrela (eixo vertical) é apresentada juntamente com a sua temperatura superficial (na horizontal). A posição da estrela no diagrama fornece informações sobre sua massa, tamanho e estágio evolutivo. Créditos: Kepler de Oliveira (2013).

Como pode ser visto da figura 1.1, as estrelas da SP possuem uma relação entre luminosidade e temperatura superficial, o que aponta que a massa é um parâmetro fundamental das estrelas da SP, sobrepujando diferenças de rotação, campos magnéticos e composição química.

Além das cores, há outro método, mais complexo e robusto, de se estimar a temperatura superficial estelar: através da identificação de linhas espectrais. A grande vantagem é que elas não são afetadas pelo avermelhamento causado pela poeira interestelar. Simplificadamente, o fluxo estelar proveniente de camadas mais internas ao passar por sua atmosfera tem energia em determinados comprimentos de onda absorvida por moléculas ou átomos, dando origem a linhas escuras ou de absorção. A razão entre as intensidades 
Tabela 1.1 - Classificação estelar em tipos espectrais. Fonte: Friaça et al. (2003).

\begin{tabular}{|c|c|c|c|c|c|}
\hline $\begin{array}{c}\text { Tipo } \\
\text { espectral }\end{array}$ & $\begin{array}{c}T_{\text {efe }} \\
\text { Kelvin }\end{array}$ & $\begin{array}{c}\text { íons ou } \\
\text { moléculas }\end{array}$ & $\begin{array}{l}\text { Índice } \\
B-V\end{array}$ & Cor & $\begin{array}{l}\text { Estrelas } \\
\text { típicas }\end{array}$ \\
\hline $\mathrm{O}$ & $\begin{array}{l}50000 \\
25000\end{array}$ & $\begin{array}{l}\text { NII, HeI, } \\
\text { SiIV }\end{array}$ & $-0,32$ a $-0,30$ & Azul & $\begin{array}{l}\text { Mintaka, } \\
\text { Alnitak }\end{array}$ \\
\hline B & $\begin{array}{l}25000 \\
11000\end{array}$ & $\begin{array}{l}\text { HI, HeI, } \\
\text { SiIII }\end{array}$ & $-0,30 \mathrm{a}-0,01$ & Azulada & $\begin{array}{l}\text { Rigel, } \\
\text { Spica }\end{array}$ \\
\hline A & $\begin{array}{l}11000 \\
7500\end{array}$ & HI, SiII, & $-0,01 \mathrm{a}+0,30$ & Branca & $\begin{array}{l}\text { Sirius, } \\
\text { Vega }\end{array}$ \\
\hline $\mathrm{F}$ & $\begin{array}{l}7500 \\
6000\end{array}$ & $\begin{array}{l}\text { HI, FeII, } \\
\quad \text { CaII }\end{array}$ & $+0,30 \mathrm{a}+0,58$ & $\begin{array}{c}\text { Branco- } \\
\text { Amarelada }\end{array}$ & $\begin{array}{l}\text { Canopus, } \\
\text { Procion }\end{array}$ \\
\hline $\mathrm{G}$ & $\begin{array}{l}6000 \\
5000\end{array}$ & FeII, CaII, & $+0,58 \mathrm{a}+0,81$ & Amarela & $\begin{array}{c}\text { Sol, } \\
\alpha \text { Centauri }\end{array}$ \\
\hline $\mathrm{K}$ & $\begin{array}{l}5000 \\
3500\end{array}$ & $\begin{array}{l}\text { CaI, FeI, } \\
\text { CH }\end{array}$ & $+0,81 \mathrm{a}+1,40$ & Laranja & $\begin{array}{c}\text { Aldebaran, } \\
\text { Arturus }\end{array}$ \\
\hline M & $\begin{array}{l}3500 \\
2700\end{array}$ & FeI, TiO, & $>+1,40$ & Vermelha & $\begin{array}{c}\text { Antares, } \\
\text { Betelgeuse }\end{array}$ \\
\hline
\end{tabular}

de diferentes linhas espectrais indica a temperatura superficial da estrela, uma vez que as populações dos níveis de energia dependem da temperatura.

A classificação por tipos espectrais foi definida em Harvard, EUA, inicialmente por Edward C. Pickering (1846-1919) e sua assistente Williamina P. Fleming (1857-1911) e com posteriores contribuições de Antonia Maury (1866-1952) e Annie Jump Cannon (1863-1941), que propuseram um ordenamento dos espectros que ficou conhecido como classificação de Harvard, sendo que esta última pesquisadora também adicionou subdivisões decimais. Na tabela 1.1 apresentamos os principais tipos espectrais com algumas de suas respectivas características, como linhas e cores fotométricas.

A análise das linhas espectrais pode fornecer grande quantidade de informações. Além da temperatura superficial estelar, ela também pode prover a composição química, movimentos e pressão superficiais. A partir dessa última informação, podemos conhecer a luminosidade estelar intrínseca, mesmo sem conhecer a sua distância até nós. Dessa forma, as classes de luminosidade complementam os tipos espectrais, ambos constituindo uma classificação bidimensional. As principais classes são apresentadas na tabela 1.2. 
Tabela 1.2 - Classes de luminosidade Maciel (1999).

\begin{tabular}{ll}
\hline Classe & Designação \\
\hline Ia-0 & Supergigantes mais luminosas \\
Ia & Supergigantes luminosas \\
Ib & Supergigantes menos luminosas \\
II & Gigantes brilhantes \\
III & Gigantes normais \\
IV & Subgigantes \\
V & Anãs \\
VI & Subanãs \\
VII & Anãs brancas \\
\hline
\end{tabular}

\subsection{Estrelas de alta massa}

A função empírica que descreve a distribuição de massa de uma população estelar é conhecida em astronomia como IMF. A IMF para estrelas mais massivas que o Sol foi quantificada pela primeira vez por Salpeter (Salpeter, 1955). Salpeter identificou que a natureza tem preferência por formar estrelas de baixa massa, decaindo rapidamente para estrelas mais massivas. Definindo $\xi(m) \mathrm{d} m$ como a fração de estrelas com massas entre $m-\mathrm{d} m / 2$ e $m+\mathrm{d} m / 2$, a IMF é parametrizada como uma lei de potência da forma

$$
\xi(m) \mathrm{d} m \propto m^{-\alpha} \mathrm{d} m,
$$

com $\alpha=2,35$. Em estudo mais recente este valor é estimado em $\alpha=2,3$ para estrelas de massa maior que $0,5 M_{\odot}($ Kroupa, 2001).

O termo "estrelas massivas" em geral é atribuído atribuído à estrelas com massas acima de $8 M_{\odot}$, de tipos espectrais $\mathrm{O}$ e $\mathrm{B}$, entretanto o subtipo espectral $\mathrm{B}$ abrange estrelas de massas tão baixas quanto $3 M_{\odot}$ (Cox, 2000). Apesar das estrelas OB comporem aproximadamente 0,1\% das estrelas da vizinhança Solar que estão na SP (Ledrew, 2001), a sua influência no meio interestelar é profunda. Seus ventos introduzem uma grande quantidade de energia cinética e momento em seu ambiente, o que impacta a taxa de formação estelar. Além disso, são as principais contribuidoras da radiação Ultra Violeta (UV) das galáxias, que é basicamente a radiação detectada para as galáxias de maior redshift em observatórios terrestres. Devido à sua rápida evolução na SP, esses astros morrem perto de onde nasceram, e funcionam como traçadores de formação estelar recente. Após a SP, essas estrelas são as principais criadoras de elementos pesados (incluindo os necessários 
à vida). Este material processado é injetado no meio interestelar o que leva à formação de novas estrelas. Além disso, nos estágios finais de sua existência, estrelas massivas dão origem a fenômenos energéticos como supernovas e Surtos de Raios Gama (GRB).

\subsubsection{Estrelas quentes e a rotação estelar}

Estrelas não podem girar acima de sua rotação crítica, que é o limite para que as forças gravitacional e centrífuga se contrabalanceiem na superfície da estrela. A aceleração centrífuga é dada pelo produto $\Omega^{2} R$ onde $\Omega$ é a taxa de rotação angular e $R$ é distância ao eixo de rotação da estrela. Consequentemente, os efeitos da rotação devem ser mais pronunciados no equador estelar.

Notação para a taxa de rotação

Há várias maneiras de se expressar a velocidade de rotação, em termos de velocidades lineares $\left(v_{\text {crit }}\right)$ e angulares $\left(\Omega_{\text {crit }}\right)$. Esses parâmetros são expressos em função de seus valores críticos

$$
v_{\text {crit }}=\sqrt{\frac{2}{3} \frac{G M_{\star}}{R_{\mathrm{p}}}} ; \quad \Omega_{\mathrm{crit}}=\sqrt{\frac{8}{27} \frac{G M_{\star}}{R_{\mathrm{p}}^{3}}},
$$

onde $G$ é a constante da gravidade, $M_{\star}$ é a massa da estrela e $R_{\mathrm{p}}$ é o raio do polo.

O fator $3 / 2$ é originário do achatamento geométrico $\left(R_{\mathrm{eq}}=3 / 2 R_{\mathrm{p}}\right)$ para a aproximação de rotação crítica de um corpo rígido, conhecido como aproximação de Roche. Nesta aproximação, superfícies equipotenciais são descritas, considerando a força centrífuga gerada pela rotação em adição à gravitacional. Rivinius et al. (2013) destaca que a descrição da taxa de rotação em termos dessas quantidades só faz sentido na aproximação de Roche. Os autores sugerem que a rotação seja expressa através da quantidade $W$, definida em termos da velocidade equatorial orbital

$$
\begin{gathered}
W=\frac{v_{\text {rot }}}{v_{\text {orb }}} ; \\
v_{\text {orb }}=\sqrt{\frac{G M_{\star}}{R_{\text {eq }}}} ; \quad \Omega_{\text {crit }}=\sqrt{\frac{G M_{\star}}{R_{\text {eq }}^{3}} .}
\end{gathered}
$$

A vantagem dessa descrição é que ela independe dos detalhes de como a estrela gira (rotação diferencial ou rígida) e $W$ diretamente define a velocidade extra necessária para uma dada estrela colocar material em órbita. 


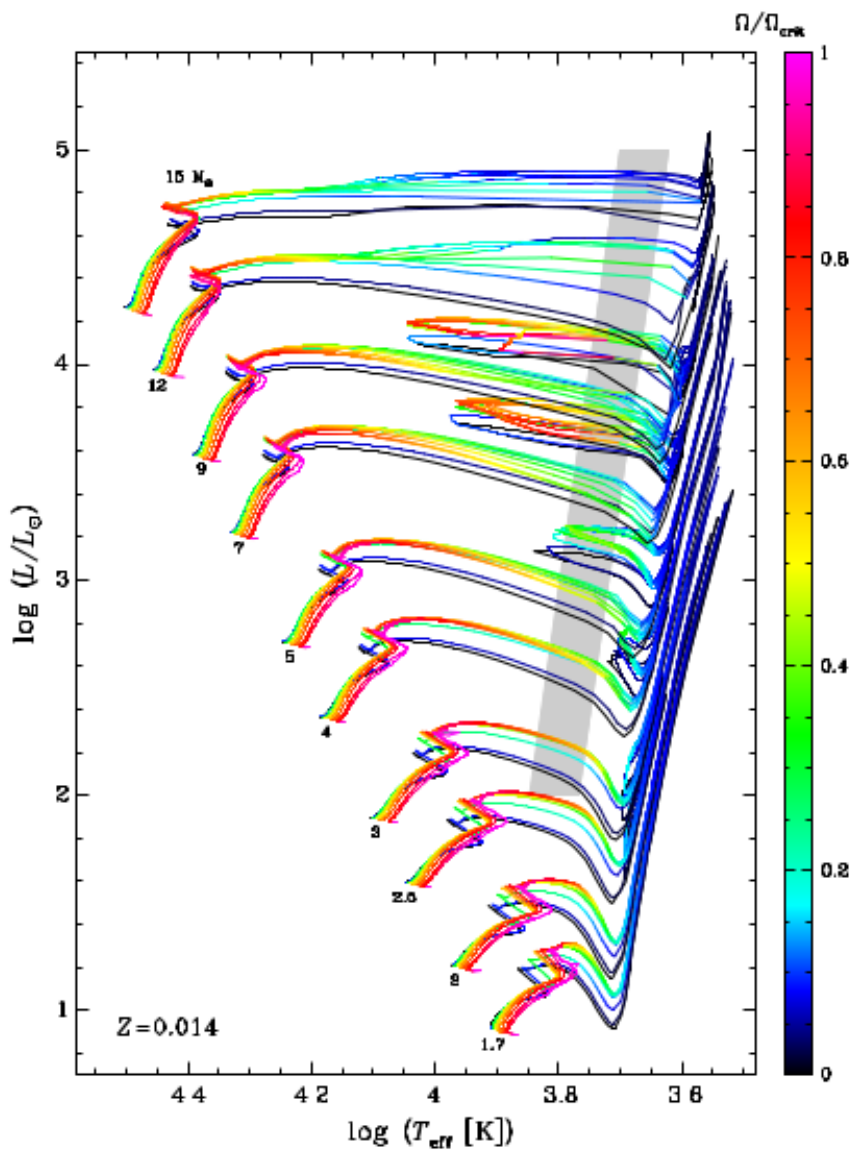

Figura 1.2: Diagrama HR para diferentes razões entre $\Omega / \Omega_{\text {crit }}$ (a escala é apresentada à direita), para estrelas de metalicidade $Z=0,014$. Para cada massa, a ordem das curvas na sequência principal de idade zero (ponto inferior esquerdo das curvas) vão da menor para a maior velocidade de rotação inicial da esquerda para a direita. Fonte: Georgy et al. 2013.

As relações de $W$ com o formalismo de rotatores rígidos usualmente utilizados na literatura são

$$
W=\sqrt{2\left(\frac{R_{\mathrm{eq}}}{R_{\mathrm{p}}}-1\right)},
$$

e,

$$
\frac{\Omega}{\Omega_{\text {crit }}}=\sqrt{\frac{27}{8} \frac{W^{2}}{\left(1+0.5 W^{2}\right)^{3}}} .
$$

A rotação estelar pode alterar o esquema evolutivo de estrelas de alta massa de várias maneiras diferentes. Por exemplo, para uma estrela cujo núcleo possui rotação superior em comparação com os envelopes externos, o equilíbrio é perturbado gerando instabilidades internas, ocasionando o transporte de elementos químicos e de momento angular para camadas mais elevadas (Georgy et al., 2013). Além disso, a rotação afeta a luminosidade e o tempo de vida na sequência principal (veja Fig. 1.2). Outros efeitos importantes originados pela rotação estelar são: 
- Achatamento geométrico: O raio do equador aumenta como consequência da alta rotação estelar. Este efeito foi diretamente detectado por estudos interferométricos das estrelas Altair (Monnier et al., 2007) e Achenar (Domiciano de Souza et al. 2003; Carciofi et al. 2008) entre outras;

- Escurecimento gravitacional ou de von Zeipel (von Zeipel, 1924): este efeito é caracterizado pelo diminuição do fluxo no equador, e aumento nos polos, como resultado da baixa gravidade efetiva. Este efeito já foi observado por interferometria (Domiciano de Souza et al., 2012).

Os efeitos da rotação eram estudados espectroscopicamente através do alargamento de linhas fotosféricas. Mas hoje sabemos que as determinações espectroscópicas são problemáticas. Cranmer (2005) e Townsend et al. (2004), por exemplo, apontam que as determinações das velocidades projetadas, $v$ sen $i$, são subestimadas quando o efeito do escurecimento gravitacional não é considerado, principalmente para valores $v_{\text {eq }} / v_{\text {crit }} \gtrsim 0,8^{1}$. Isto ocorre devido à contribuição da luminosidade proveniente de regiões de menor velocidade (polos quentes) ser mais significativa do que a radiação oriunda de regiões equatoriais, que possuem velocidade mais elevada. Por este motivo as estimativas de $v$ sen $i$ são usualmente consideradas como limites inferiores desses valores.

\subsection{Estrelas Be}

\subsubsection{Estrelas quentes peculiares}

Estudos espectroscópicos mostram que uma fração das estrelas OB apresentam características espectrais peculiares, das quais uma fração considerável exibe emissões em linha e no contínuo provenientes de seus ambientes circunstelares. Dentro desse cenário, um efeito que tem merecido maior atenção nos últimos anos é o papel da binaridade em estrelas peculiares, o que permite a troca de massa e momento angular entre as duas componentes. É importante ressaltar que pelo menos $44 \%$ das estrelas OB se encontram em sistemas binários ou múltiplos (Mason et al. 2009; Sana e Evans 2011) e descobertas recentes indicam que estrelas Be binárias podem ser mais frequentes do que se acreditava (Klement et al., 2017).

\footnotetext{
${ }^{1} v_{\text {eq }}$ é a velocidade equatorial.
} 
A seguir apresentamos um sumário de estrelas quentes peculiares (Rivinius et al., 2013):

- SPB: estrelas de tipo B recentes de pulsação lenta, com períodos de algumas horas;

- $\beta$ Cep: estrelas de tipo B que possuem variabilidade fotométrica de períodos de alguns dias;

- Ae/Be de Herbig: estrelas pré-SP com disco de acreção;

- Supergigantes OB: estrelas pós-SP que inclui subtipos estelares Luminosas Azuis variáveis (LBV), Wolf-Rayet (WR), P cyg e VV Cep;

- Oe: estrelas de tipo com com ventos fortes e que exibem linhas de emissão;

- Ricas em He: estrelas de tipo B recentes com linhas incomumente fortes de He nãoionizado. Frequentemente essas linhas estão associadas a intensos campos magnéticos na fotosfera;

- Bn: estrelas de tipo espectral B que exibem perfis linha alargados mas sem sinais de emissão, habitualmente interpretadas como rotatores rápidos vistos de lado;

- $\mathbf{B}[\mathbf{e}]$ : é um grupo não-homogêneo de estrelas de tipo espectral B onde são identificadas linhas de Balmer e proibidas em emissão (Lamers et al., 1998). Linhas proibidas em emissão não são observadas em estrelas Be;

- Be Clássica: estrelas de tipo B que estão na SP e que exibem ou exibiram em seus espectros linhas de emissão. Uma fração de 30\% a $70 \%$ das estrelas B são Be (Martayan et al., 2007).

Propriedades espectrais que caracterizam as estrelas OB ativas são apresentadas na tabela 1.3.

\subsubsection{Contexto Histórico}

Há aproximadamente 150 anos atrás, em 23 de agosto de 1866, o Padre A. Secchi, diretor do observatório do Collegio Romano, reportou ao editor do "Astronomische Nachrichten" uma observação de $\gamma$ Cas (B0.5 IV), na linha de hidrogênio $H \beta$ (4861 A) que exibia "une particularité curieuse [...] une ligne lumineuse très belle et bien plus brillante que 
Tabela 1.3 - Propriedades espectrais selecionadas de estrelas quentes peculiares. As linhas de emissão são formadas em: (1a) disco de decréscimo equatorial, (1b) disco de acreção, (1c) nuvens em corrotação. Outras propriedades incluem: (2) variações de perfis de linha de baixa ordem, (3) pulsação radial ou de curto período, (4) rotação rápida, (5) campo magnético em larga escala e (6) anormalidades de abundâncias superficiais. É importante ressaltar que as características apresentadas na tabela representam uma propriedade estatisticamente esperada e não devem ser interpretadas como requisitos necessários. Adaptado de Porter e Rivinius (2003), originário de Baade et al. (2003).

\begin{tabular}{lccccccccc}
\hline & \multicolumn{1}{c}{ Propriedade } & \multicolumn{1}{c}{ geral } & \multicolumn{3}{c}{} \\
Grupo estelar & $1 \mathrm{a}$ & $1 \mathrm{~b}$ & $1 \mathrm{c}$ & 2 & 3 & 4 & 5 & 6 \\
\hline Be Clássica & $\sqrt{ }$ & - & - & $\sqrt{ }$ & - & $\sqrt{ }$ & - & - \\
Ae/Be de Herbig & - & $\sqrt{ }$ & - & - & - & - & - & - \\
Rica em He & - & - & $\sqrt{ }$ & - & - & $\sqrt{ }$ & $\sqrt{ }$ & $\sqrt{ }$ \\
SPB & - & - & - & $\sqrt{ }$ & - & - & - & - \\
$\beta$ Cep & - & - & - & - & $\sqrt{ }$ & - & - & - \\
Bn & - & - & - & - & - & $\sqrt{ }$ & - & - \\
Bp e anormal em He & - & - & - & - & - & - & $\sqrt{ }$ & $\sqrt{ }$ \\
\hline
\end{tabular}

tout le reste du spectre". Esta foi posteriormente reconhecida como a primeira observação de uma estrela $\mathrm{Be}^{2}$.

O trabalho de Struve (1931) foi o primeiro a diferenciar estrelas Be das que apresentavam perfis $\mathrm{P}$ Cygni e de estrelas como $\beta$ Lyr (B8), que teve seu espectro associado à binaridade. Seu trabalho foi pioneiro, reconhecendo estrelas Be como sendo objetos em alta rotação, assumindo uma forma lenticular e que ejetam matéria levando à formação de um anel que rotaciona em torno da estrela, originando linhas de emissão, o que levou à unificação entre os conceitos de estrelas shell e Be. Estrelas shell possuem linhas de Balmer em emissão com centro em absorção acentuada, mais do que o esperado de linhas fotosféricas normais. Podem conter emissão de duplo pico em ambos lados do centro da absorção. Segundo a concepção de Struve, estrelas shell são Be vistas de lado, i.e., observadas através do material circunstelar, o que ocasiona linhas de absorção acentuada. A Fig. 1.3 apresenta uma visão esquemática dessa ideia. A interpretação de Struve foi bem aceita, de forma geral, até ser posta em dúvida nas décadas de 70 e 80, quando um número de modelos opostos surgiram.

Estrelas Be não são raras e , à selhança de $\gamma$ Cas, observada por Secchi, algumas

\footnotetext{
${ }^{2}$ Reproduzimos a seguir o comentário de Rivinius et al. (2013) sobre essa descoberta: "In the heyday of nationalism, this was communicated in French language to a German Journal by an Italian astronomer, working at the international organization of the time, the Vatican".
} 


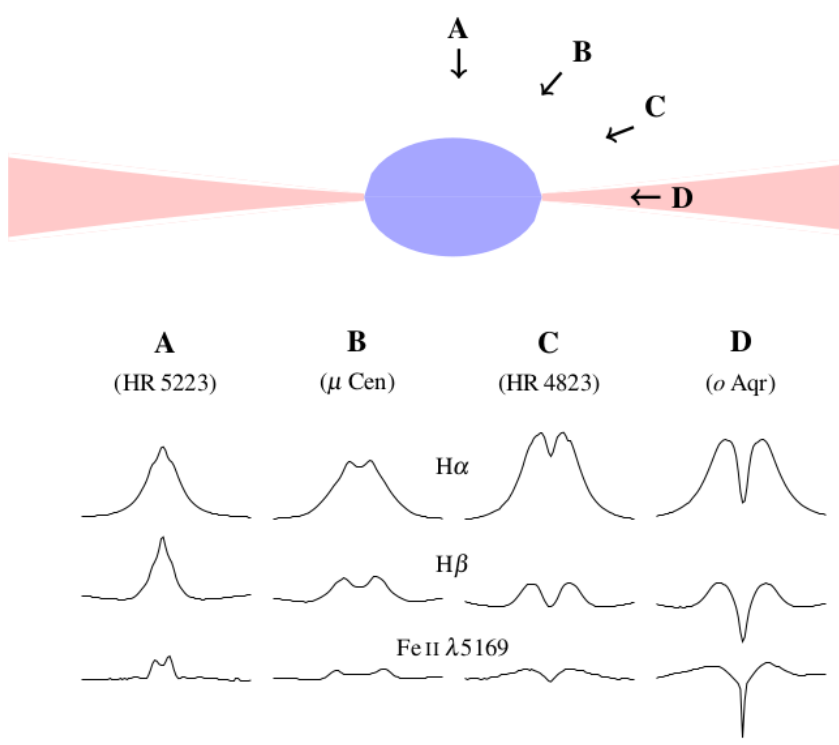

Figura 1.3: Figura esquemática de uma estrela Be em rotação crítica e com disco. Na parte inferior são mostrados como os perfis de linha variam entre uma estrela vista a partir do polo até uma estrela vista de lado, também conhecida como estrelas Be shell.

das estrelas Be estão entre as estrelas mais brilhantes do céu visível, o que as tornam interessantes para astrônomos amadores ${ }^{3}$.

A definição clássica de estrelas Be, ainda bastante utilizada, foi inicialmente sugerida por Jaschek et al. 1981 com uma pequena adaptação ${ }^{4}$, e posteriormente popularizada por Collins (1987) como: "estrelas de tipo B não supergigante cujo espectro possui, ou possuiu em algum momento, uma ou mais linhas de Balmer em emissão". O problema com essa classificação é a sua abrangência, que inclui, por exemplo, estrelas de tipo espectral B cujo material circunstelar possui densidades superiores à $10^{-13} \mathrm{~g} \mathrm{~cm}^{-3}$. Em uma orientação mais moderna, estrelas Be clássicas são estrelas de tipo espectral B em altas rotações, com pulsações não radiais, e acompanhada por um disco gasoso Kepleriano auto-ejetado, cuja evolução é governada pela viscosidade. O tema dos discos e sua física será discutido mais abaixo, no capítulo 2.

$\mathrm{Na}$ vizinhança solar, uma estimativa média das medidas de $W$ para as estrelas Be é de $\bar{W}=0,8$ (Rivinius et al., 2013). A distribuição dos valores medidos é da ordem da incerteza observacional e não possui dependência com temperatura ou gravidade efetiva. $\mathrm{O}$ valor mínimo de $W$ para uma estrela Be se tornar Be é de 0,7 , e como $W$ não depende da

\footnotetext{
${ }^{3}$ Um exemplo interessante é o site http://basebe.obspm.fr/basebe/, que reúne dados de estrelas Be, AeBe de Herbig e supergigantes $\mathrm{B}[\mathrm{e}]$ obtidos por astrônomos amadores e profissionais.

${ }^{4}$ Alterando o termo original "linhas de hidrogênio" para "linhas de Balmer".
} 
$T_{\text {ef }}$, este mínimo não deve depender fortemente da massa (Rivinius et al. 2006; Townsend et al. 2004; Meilland et al. 2012; Frémat et al. 2005).

Uma questão debatida nas últimas três décadas, se refere à variabilidades com períodos entre 0,5 e 3 dias ser causada por pulsações ou rotação (veja Porter e Rivinius 2003). Rivinius et al. (2003) argumenta que, para a maioria das estrelas Be de tipo recente, a variabilidade observada é causada por pulsações não-radiais de baixa ordem. Importantes contribuições foram feitas por missões de satélites fotométricos, o que alavancou avanços recentes na astro-sismologia em geral (Aerts et al., 2010). Walker et al. (2005) foi o primeiro a relatar multi-periodicidade para a estrela Oe $\zeta$ Oph $(\mathrm{O} 9.5 \mathrm{~V})$, utilizando fotometria espacial. O primeiro relato de pulsações de baixa amplitude $(\approx 1 \mathrm{mmag})$ na estrela Be de tipo tardio $\beta$ CMi (B8 Ve) foi feito por Saio et al. (2007).

Muitas das estrelas peculiares definidas na seção 1.3.1 possuem campos magnéticos. O estudo de campos magnéticos em estrelas quentes tem recebido grande atenção recente com levantamentos como o MIMES (Wade et al. 2012, 2016) e BOB (Morel et al., 2015). Curiosamente, estrelas Be não têm nenhuma detecção confiável de campo magnético (as medidas reportadas são todas compatíveis com um valor nulo, dentro de $3 \sigma$ ).

\subsubsection{Observáveis de estrelas Be}

Uma das características observacionais mais marcantes de estrelas Be é a sua variabilidade, em basicamente todos os observáveis e em várias escalas de tempo, oferecendo a possibilidade de entendimento de vários fenômenos astrofísicos diferentes e suas interações. A natureza das variações pode ser periódica, quase periódica ou irregular (Mennickent et al. 1994 e Sterken et al. 1996). As variabilidades de curto período (alguns dias) estão ligadas às pulsações não-radiais (Baade 1998; Baade et al. 2016), enquanto que as de longo período estão vinculadas à presença do disco e à ejeção de massa pela estrela (Haubois et al., 2012).

A distribuição de energia espectral, $\mathrm{SED}^{5}$, de uma estrela Be é a combinação da emissão fotosférica e da absorção e reprocessamento da radiação estelar pelo disco (veja Fig. 1.4). A contribuição de cada componente varia muito conforme o ângulo de visada e o comprimento de onda.

A emissão do disco pode ser melhor entendida em termos de uma pseudofotosfera, que é uma região do disco que é bastante opaca à radiação. Dessa forma, o disco possui dois

\footnotetext{
5 Spectral Energy Distribution.
} 


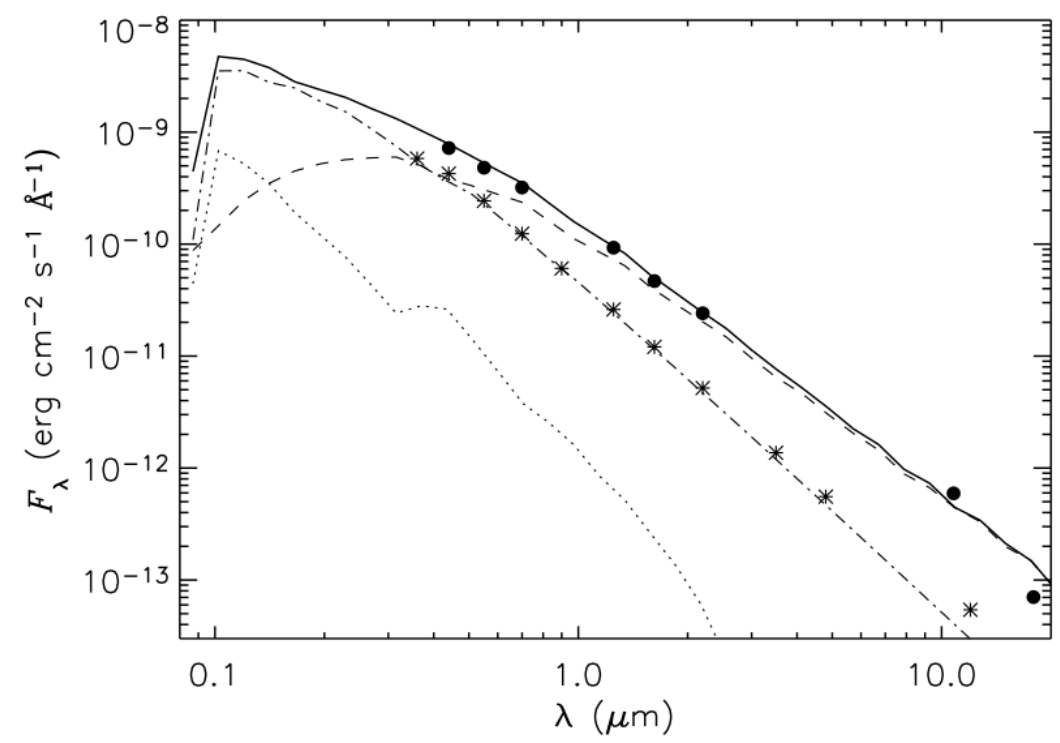

Figura 1.4: Melhor modelo ajustado à fase ativa da estrela Be $\delta$ Scorpii (linha sólida) é mostrado juntamente com a SED observada (círculos cheios). Os dados fotométricos da fase pré-ativa também são mostrados (asteriscos) para comparação. As outras linhas correspondem aos fluxos estelar não processado, espalhado e emitido (respectivamente, linhas pontilhada, tracejada e pontilhada-tracejada). Adaptado de Carciofi et al. (2006).

perfis de brilho superficial: uma região interna opticamente espessa, que se comporta como uma pseudofotosfera cujo raio, $\bar{R}$, depende do comprimento de onda, e uma região externa opticamente fina (veja Fig. 1.5a). O caso onde $\bar{R} \leq R_{\star}$ (onde $R_{\star}$ é o raio da estrela) corresponde a um disco inteiramente opticamente fino na direção da linha de visada (Fig. 1.5b). A situação de $\bar{R} \geq R_{\text {disco }}$ (Fig. 1.5c) tipicamente ocorre quando o disco é muito pequeno (ou seja, truncado por algum processo físico) e/ou em comprimentos de onda muito longos (e.g., na região do submilimétrico ou do rádio).

Outra forma de se observar o efeito da pseudofotosfera é através da Fig. 1.6, que ilustra a região da formação da emissão do disco no contínuo para várias bandas e duas densidades do disco. O que é apresentado é o fluxo integrado normalizado pelo fluxo total como função da distância radial à estrela. Nota-se que diferentes bandas possuem regiões de formação distintas. Por exemplo, $80 \%$ do fluxo na banda $V$ é proveniente de uma região de 1.8 a $2.5 R_{\star}$, dependendo da densidade do disco. Por outro lado, o mesma fração do fluxo em $60 \mu \mathrm{m}$ é originária de uma região bem mais extensa, entre 5 e $15 R_{\star}$.

As características da emissão proveniente de uma estrela Be dependem também da inclinação relativa do disco com referência à linha de visada:

- Estrelas vistas a partir do polo: Como o disco é mais frio que a fotosfera, a 
optically thick

optically thin

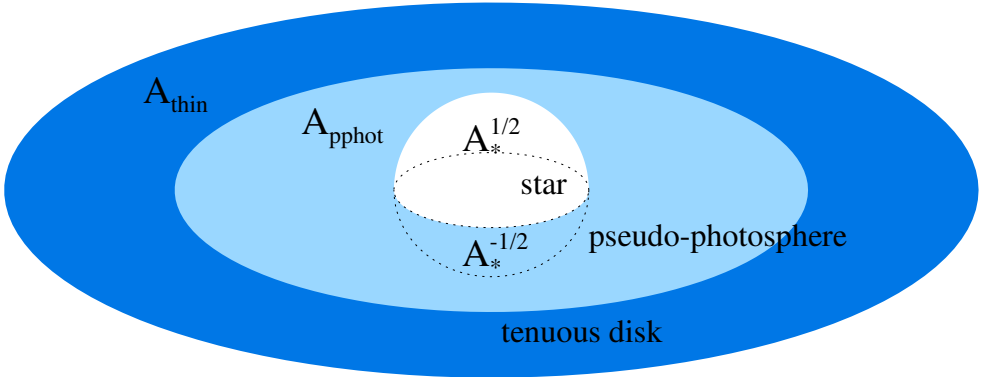

(a) general case

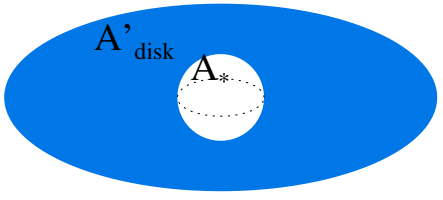

(b) tenuous disk

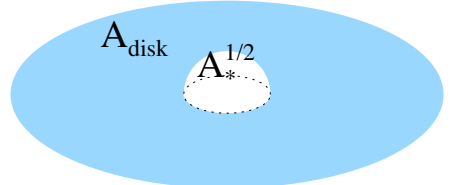

(c) truncated pseudo-photosphere

Figura 1.5: Representação esquemática das componentes do disco, para os três casos possíveis: (a) presença de ambos regimes de emissão, onde $R_{\star}<\bar{R}<R_{\mathrm{d}}$; (b) o caso opticamente fino, onde $\bar{R} \leq R_{\star}$; e (c) pseudofotosfera, onde $\bar{R} \leq R_{\mathrm{d}}$. Figura adaptada de Vieira et al. 2015 .
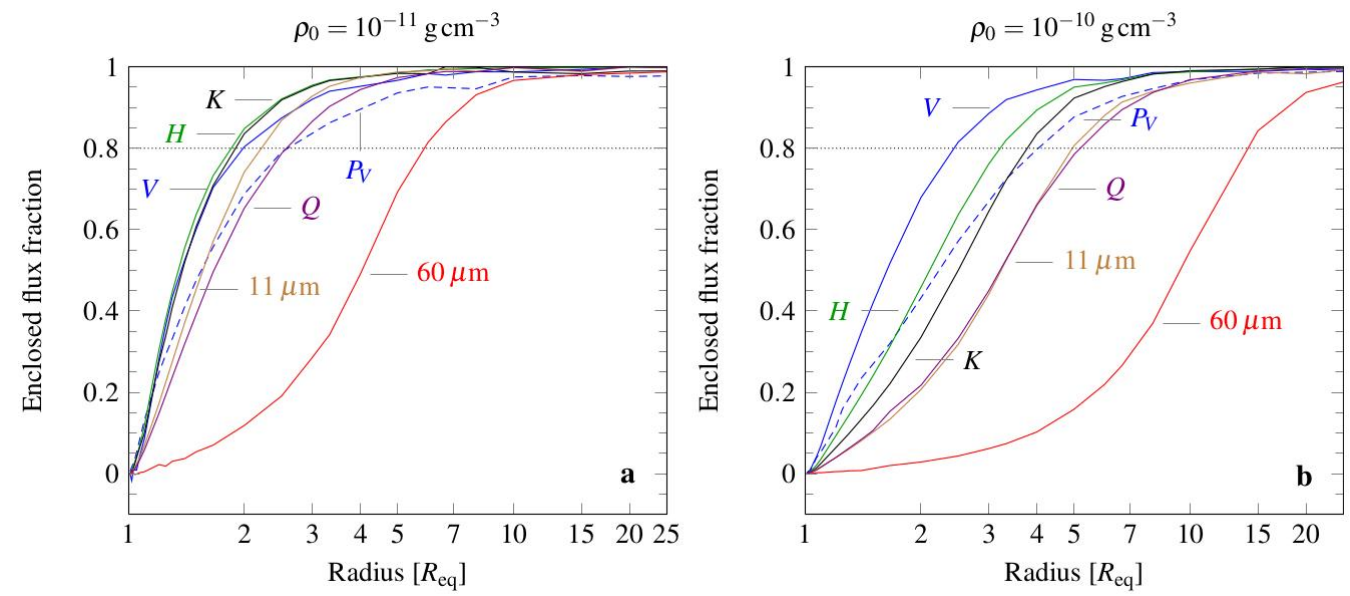

Figura 1.6: Locais de formação da emissão do contínuo para várias bandas fotométricas e para o fluxo polarizado na banda $V, P_{\mathrm{V}}$, expressos na contribuição do disco acumulada como função crescente do raio para duas densidades diferentes. O fluxo estelar foi subtraído para que cada curva comece do zero. A linha pontilhada marca $80 \%$ do fluxo total, o que corresponde ao fluxo integrado dentro da largura a meia altura para um perfil de emissão gaussiano. Os dados foram computados com o HDUST (veja seção 2.3.2 para mais detalhes) para o modelo de referência de Faes et al. (2013), para um disco visto a $i=30^{\circ}$. Crédito: Rivinius et al. (2013). 

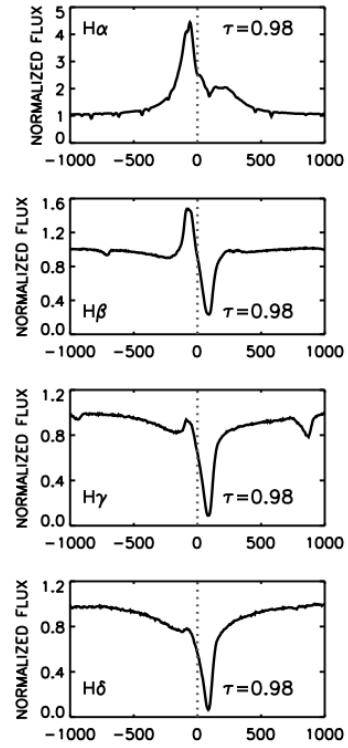
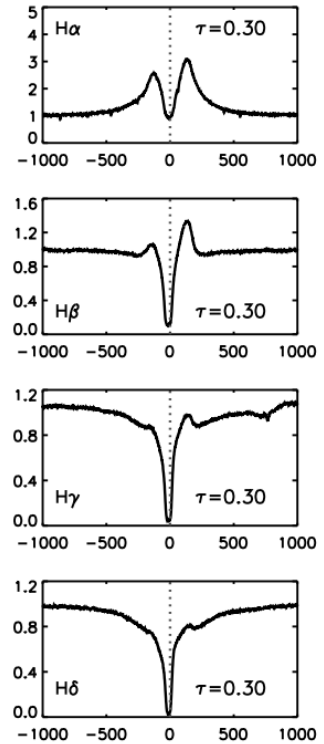
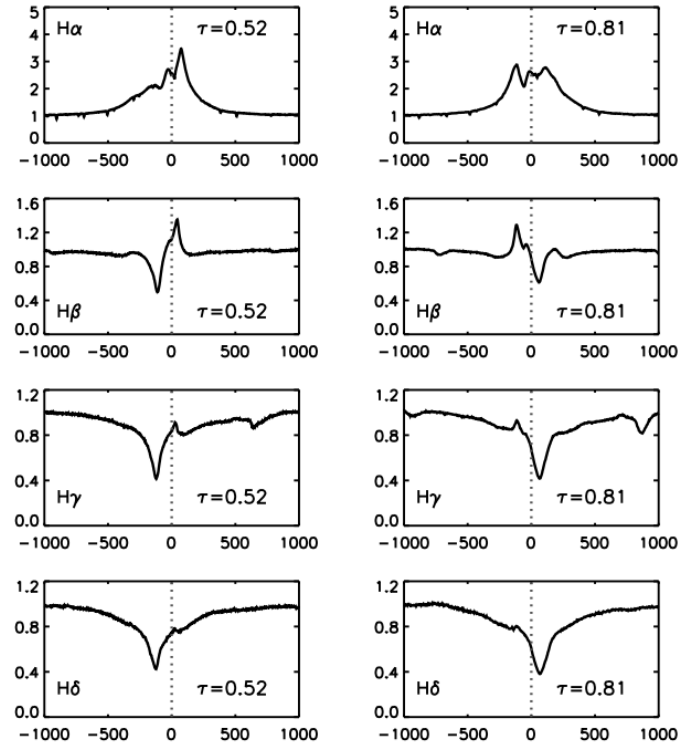

Figura 1.7: Espectro óptico da estrela Be $\zeta$ Tauri observado em diferentes fases do ciclo $V / R$. Da esquerda para a direita: fases $V / R, \tau=0.98,0.30,0.52$ e 0.81 ; de cima para baixo: linhas de Balmer sucessivas de $\mathrm{H} \alpha$ a $\mathrm{H} \delta$. A linha pontilhada marca a velocidade de repouso $\left(v_{\mathrm{rad}}=0 \mathrm{Km} \mathrm{s}^{-1}\right)$. Crédito: Escolano et al. (2015).

SED será uma combinação da emissão estelar com um excesso em comprimentos de onda mais longos oriundo do disco. O peso relativo das componentes dependerá do tamanho da pseudofotosfera e do comprimento de onda: o excesso em geral não ultrapassa meia magnitude na região do visível, chegando a dominar o fluxo total na região do infravermelho próximo e comprimentos de onda maiores (veja Haubois et al. 2012, Sigut e Patel 2013).

- Estrelas vistas de lado: neste caso há uma competição entre a absorção da radiação fotosférica e a emissão pelo disco. Se a pseudofotosfera é pequena, o resultado é uma diminuição no fluxo líquido do sistema, como o observado em estrelas shell. Na situação oposta, a área emissora mais extensa compensa a diminuição no fluxo fotosférico, produzindo um aumento no fluxo resultante.

\section{Espectroscopia}

Uma das consequências do material do disco atuar como uma pseudofotosfera muito maior que a fotosfera estelar é que os espectros de Be apresentam linhas de emissão. Diferentes padrões observados nas linhas em emissão de estrelas Be podem ser explicados por discos e diferentes ângulos de inclinação, como já previsto por Struve (1931) (veja Fig. 


\section{$1.3)$.}

Além da simples constatação da presença ou ausência de disco pela existência de linhas em emissão ou absorção, variações nas propriedades físicas do disco estão associadas à variabilidade nas larguras equivalentes e nos perfis das linhas. A principal linha de diagnóstico é a de $H \alpha(6563 \AA ̊)$, que corresponde à transição eletrônica do átomo de hidrogênio de $n=3$ para $2^{6}$.

Aproximadamente metade das estrelas Be apresentam uma variação de perfil de linha bem mais complexo que os apresentados na Fig. 1.3, exibindo as chamadas variações V/R (Okazaki, 1991). Isto deve-se ao fato de que o disco encontra-se em rotação Kepleriana, de maneira que uma parte da emissão, associada ao material que se afasta de nós, é desviada para o vermelho, enquanto que a outra parte para o azul. Valores diferentes da unidade da razão $V / R$ entre os picos de emissão no azul ( $V$ de violet) e vermelho ( $R$ de red) podem ser causados pela presença de inomogeneidades nos discos. No caso da estrela $\zeta$ Tauri, por exemplo, (Fig. 1.7) essas inomogeneidades são formadas por ondas de densidade no disco que precessionam com período aproximado de 3,9 anos (Carciofi et al., 2009). Outras fontes de inomogeneidade associadas a períodos distintos ou mesmo aperiódicos são ejeções assimétricas de material estelar ou pela presença de uma estrela secundária.

\section{Interferometria e espectro-interferometria}

A interferometria é uma técnica que permitiu, e ainda permite, grandes avanços do conhecimento acerca de vários fenômenos astrofísicos. Dois exemplos importantes, são as recentes observações de formação planetária, utilizando o $\operatorname{ALMA}^{7}$ (e.g., Andrews et al. 2016), e o esforço de observar diretamente o ambiente imediato ao horizonte de eventos de Sagittarius $A^{*}$, o buraco negro no centro da Galáxia, pela iniciativa $\mathrm{EHT}^{8}$ (Fish et al., 2016).

A vantagem dessa técnica é que ela nos permite detectar estruturas com resolução angular de um telescópio cuja abertura é equivalente à separação entre os telescópios (ou radiotelescópios) componentes. A resolução obtida com essa técnica é maior do que qualquer outro instrumento astronômico, capaz de imagear o céu com um nível de detalhe

\footnotetext{
${ }^{6} n$ é o número quântico principal.

7 Atacama Large Millimetre/submillimetre Array.

${ }^{8}$ Event Horizont Telescope.
} 


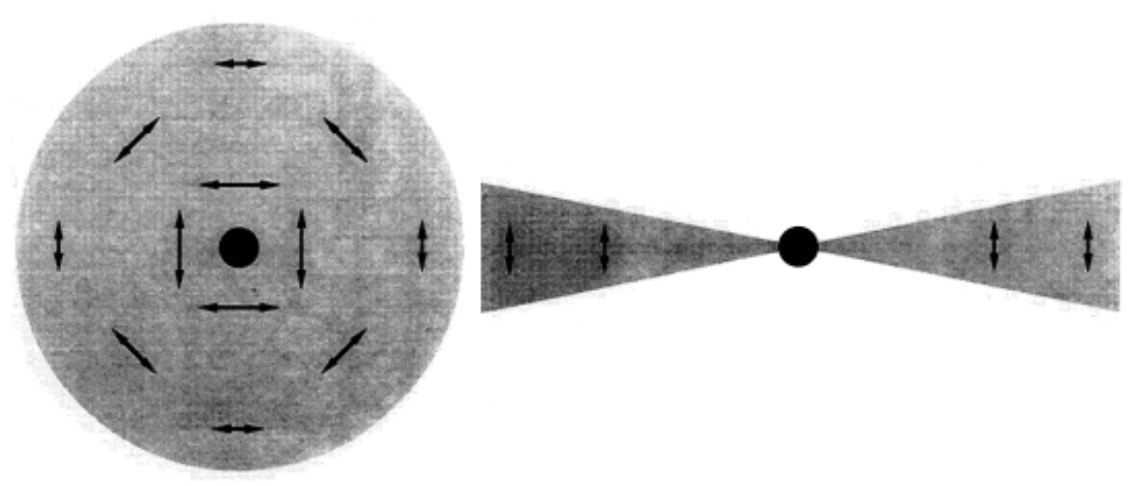

Figura 1.8: Esquerda: Figura esquemática que mostra os vetores de polarização gerados por um envoltório circunstelar esfericamente simétrico. Note que a polarização líquida será nula pois cada vetor se cancelará com outro que está exatamente um quadrante de distância. Direita: O envoltório possui a forma de um disco equatorial. Neste caso, os vetores não se cancelam, e a polarização líquida é perpendicular ao disco (Adaptado de Wood et al. 1996).

medido em microarcsec, para os interferômetros do $\mathrm{ESO}^{9}$ no infravermelho próximo.

Quirrenbach et al. (1997) utilizou interferometria combinada com polarimetria para concluir que a geometria do envoltório circunstelar de Be é melhor explicada por um disco equatorial geometricamente fino. Outro resultado importante é a observação da estrela Achenar em uma fase que não possuía disco, o que permitiu a determinação de seu achatamento equatorial, possibilitando precisar sua rotação em $96 \% \pm 3 \%$ da velocidade crítica (Domiciano de Souza et al. 2003; Domiciano de Souza et al. 2012).

Mais recentemente, pôde-se demonstrar diretamente que a dinâmica de discos de Be é, para todos os efeitos, Kepleriana, com o auxílio da espectro-interferometria e espectroastrometria (Carciofi 2011; Oudmaijer et al. 2011; Štefl et al. 2011; Meilland et al. 2012; Wheelwright et al. 2012). Este é um importante resultado, que estabelece a viscosidade como o mecanismo de condução do outflow, fortalecendo o modelo de decréscimo viscoso (veja a seção 1.4 para maiores detalhes).

\section{Polarimetria e espectropolarimetria}

A polarimetria pode ser aplicada em várias áreas da Astronomia, por exemplo, para o estudo de Núcleos Ativos de Galáxias (AGNs), GRB, supernovas, gás e poeira do meio interestelar, campos magnéticos estelares, discos de debris, material circunstelar, entre outras.

\footnotetext{
${ }^{9}$ European Southern Observatory.
} 
Em estrelas quentes, o mecanismo que gera uma polarização não-nula é o espalhamento Thomson por elétrons livres, que produz uma polarização perpendicular ao plano de espalhamento. Estrelas sem envoltório circunstelar ou com envoltórios esfericamente simétricos não possuem polarização intrínseca, pois como não há um plano de espalhamento preferencial, as polarizações criadas se cancelam (Fig. 1.8, à esquerda). No caso de um envoltório fortemente assimétrico, a polarização gerada pelos espalhamentos ao longo da direção mais alongada não se anula, gerando uma polarização intrínseca líquida (Fig. 1.8, à direita).

Do ponto de vista observacional, a polarimetria pode ser feita em banda larga (e.g., com a aplicação dos filtros UBVRIJHK) quanto por espectropolarimetria. Esta técnica observacional possui uma capacidade de diagnóstico bastante interessante, por exemplo, pode-se obter a direção do disco projetada no céu de uma estrela Be, sem que seja necessário imagear o sistema (ver Bednarski 2016) para uma explicação detalhada de como isso pode ser feito).

No caso específico de estrelas Be, a polarização gerada pela presença do disco pode chegar a 2\% da radiação proveniente da estrela (Haubois et al., 2014). Medidas polarimétricas foram e são muito importantes para o estudo de estrelas Be.

\subsection{Modelo de decréscimo viscoso}

Nas seções anteriores foram apresentadas as técnicas observacionais habitualmente empregadas no estudo de estrelas Be e seus discos. Apesar de existirem indícios espectroscópicos de que os discos de Be rotacionam de forma Kepleriana (por exemplo, os perfis de linhas Central Quasi-Emission (CQE) em estrelas shell; Hanuschik 1995), apenas com trabalhos espectro-interferométricos mais recentes evidências mais precisas e confiáveis foram obtidas (Meilland et al. 2012, Kraus et al. 2012, Wheelwright et al. 2012). Até agora, a única teoria que atende aos vínculos observacionais e é capaz de reproduzir os observáveis satisfatoriamente, é o modelo de decréscimo viscoso, Disco de decréscimo viscoso (VDD), apresentado por Lee et al. (1991) com contribuições posteriores de diversos autores (e.g. Bjorkman 1997; Porter 1999; Okazaki 2001; Bjorkman e Carciofi 2005; Carciofi 2011; Rímulo et al. 2018).

Este modelo é semelhante ao utilizado na modelagem de discos proto-estelares (Pringle, 1981), com a diferença de que estes são discos de acreção em que a matéria difunde-se para 
dentro, enquanto que os de Be são de decréscimo, com a matéria formando um lento outflow gasoso. A sua formulação sempre foi baseada no formalismo de alpha-disk, onde a viscosidade cinemática é parametrizada pela quantidade $\alpha$ (Shakura e Sunyaev, 1973). No VDD, supõe-se um mecanismo que injeta material na base do disco a uma velocidade Kepleriana. Torques viscosos resultantes da rotação Kepleriana diferencial são responsáveis por transportar momento angular da base do disco para fora, o que permite que partículas atinjam órbitas cada vez mais afastadas. Discos de estrelas Be são ambientes altamente ionizados onde efeitos Equílibrio Termodinâmico Não Local (NLTE) são importantes. Radialmente, são controlados pela viscosidade e verticalmente sustentados por pressão, o que confirma que a solução completa da estrutura do disco também é determinada pelo equilíbrio radiativo (e depende da solução da transferência radiativa). O modelo de VDD tem se mostrado consistente com múltiplas modelagens de estrelas individuais (veja Rivinius et al. 2013 para uma revisão).

Apesar do grande sucesso do modelo, há algumas questões em aberto, entre as mais importantes estão: uma descrição apropriada para o mecanismo de viscosidade, e a compreensão do mecanismo de ejeção de massa da estrela para o disco. O entendimento de ambos processos são cruciais para entendermos o variabilidade de disco dessas estrelas, e potencialmente, de discos astrofísicos em geral.

Estudos dinâmicos do modelo VDD são recentes e raros, tendo o grupo de estudos de estrelas Be do IAG oferecido contribuições relevantes nessa área. Dada a importância desse tema para esta dissertação, o assunto será abordado com maior detalhe no Cap. 2.

\subsection{Relevância astrofísica de estrelas Be}

O estudo de estrelas Be busca entender os mecanismos físicos nelas presentes, bem como em seus discos. A compreensão da teoria de discos circunstelares tem uma relevância astrofísica bastante ampla, que vai além do campo de estrelas Be propriamente dito. Discos de acreção são observados em AGNs e nuvens proto-estelares. O processo de acreção também ocorre em sistemas binários quando uma das companheiras preenche o seu lóbulo de Roche e espirala material para a secundária, que pode ser um buraco negro, uma estrela de nêutrons, uma anã-branca ou uma estrela anã. Outro exemplo importante são os discos de estrelas jovens T Tauri e nas estrelas AeBe de Herbig, que estão em fase de acreção. 
Tamanha variedade de sistemas, onde a viscosidade é um parâmetro de importância fundamental, fornece a motivação para investigar a viscosidade e quantificar seu valor. Estrelas Be são laboratórios ideais para esses estudos porque seus discos não possuem poeira e apresentam uma estrutura consideravelmente mais simples do que os exemplos mencionados anteriormente. Além disso, os tamanhos de seus discos são relativamente pequenos (de algumas dezenas de raios estelares), i.e., possuem uma escala de tempo muito menor (semanas até meses) do que, por exemplo, discos protoplanetários e de AGNs. Adicionalmente, devido às suas características específicas, o estudo de estrelas Be proporciona a oportunidade única de explorar e entender muitos ramos importantes da astrofísica, como o processo de perda de massa, evolução de estrelas em alta rotação, astro-sismologia e, o que é mais importante para este trabalho, discos astrofísicos.

\subsection{Objetivos e organização do trabalho}

A dissertação se concentra no estudo das curvas de luz de uma amostra de candidatas a estrelas Be da Grande Nuvem de Magalhães que exibem claros episódios de formação e dissipação de disco, aumentando a amostras de estrela Be estudadas com o VDD dinâmico.

No capítulo 2 uma breve revisão dos estudos sobre o VDD dinâmico é apresentada, seguida da apresentação da metodologia empregada neste trabalho. No capítulo 3 apresentamos a amostra de curvas de luz analisadas, bem como uma análise estatística inicial de propriedades desses dados. Os resultados de uma modelagem inicial são discutidos no capítulo 4. As principais conclusões e perspectivas deste trabalho são traçadas nos capítulos 5 e 6 . 
Capítulo 2

\section{Discos dinâmicos de decréscimo viscoso}

No capítulo anterior fizemos uma contextualização do nosso trabalho e apresentamos resumidamente os principais conceitos envolvidos. Este capítulo é iniciado por um breve resumo histórico da modelagem dinâmica de estrelas Be, na seção 2.1, seguido pela teoria empregada na modelagem, que é introduzida na seção 2.2. A metodologia e os modelos utilizados neste trabalho são apresentados em seguida, na seção 2.3.

\subsection{Revisão histórica}

Estrelas Be são variáveis em várias escalas de tempo em diferentes observáveis. Uma compreensão completa desse comportamento dinâmico não é tarefa simples e requer um entendimento global dos processos físicos em atuação. Estudos dinâmicos do modelo de VDD são recentes e escassos na literatura. Em parte, isso é atribuído aos avanços recentes na teoria de VDD que ocorreram principalmente na última década, e também ao custo computacional elevado de simulações dinâmicas.

Jones et al. (2008) combinou códigos hidrodinâmicos 1-D e de transferência radiativa NLTE para estudar as estruturas de temperatura e densidade de um disco dinâmico e os consequentes perfis de linha em $\mathrm{H} \alpha$. Haubois et al. (2012) utilizando um disco com simetria axial e o código de transferência radiativa NLTE 3-D, HDUST, simularam a evolução temporal fotométrica de diferentes cenários de construção e dissipação de disco nas bandas $V, K$ e em comprimentos de onda de $1 \mathrm{~mm}$. Os autores analisaram a evolução temporal da densidade do disco para cenários dinâmicos diferentes que incluem: i) a construção de disco resultante de uma injeção de massa constante e longa, ii) a dissipação do disco após a perda de massa cessar e iii) cenários onde períodos de perda de massa e quiescência são 


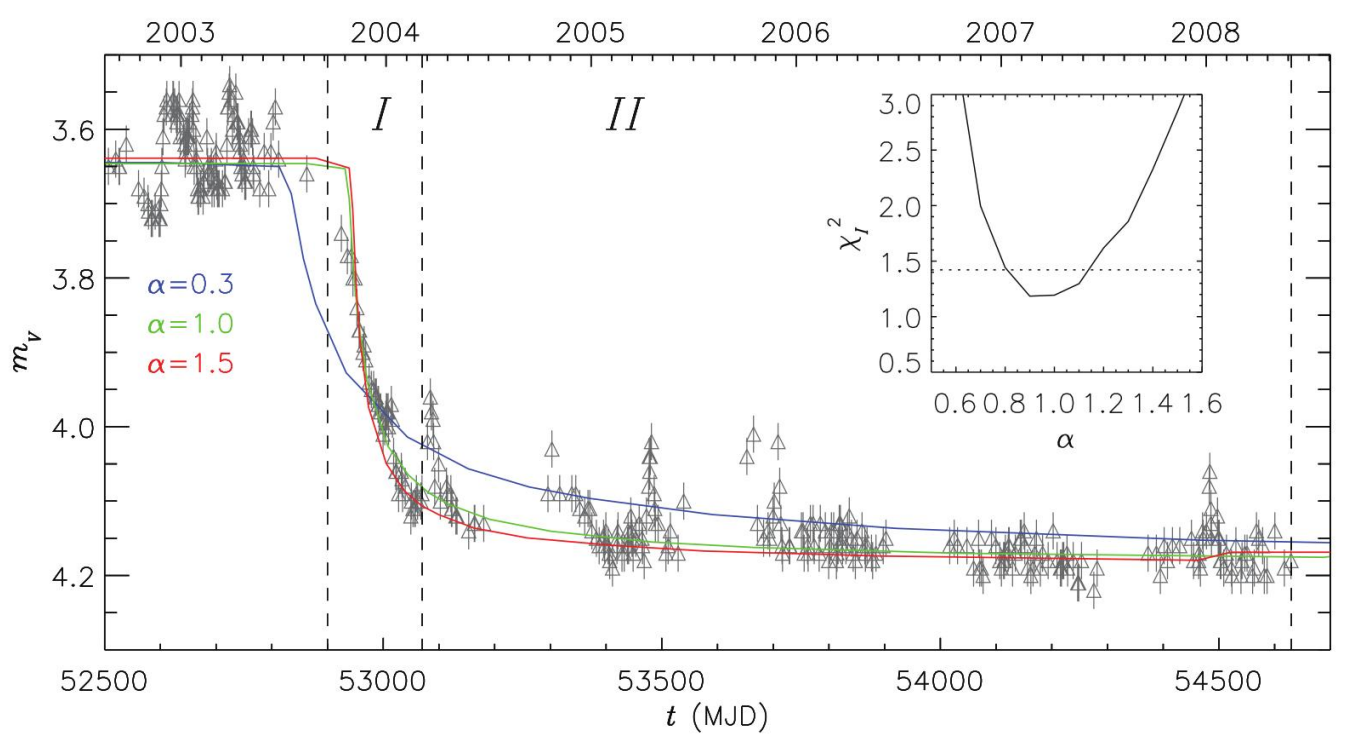

Figura 2.1: Curvas de luz na banda $V$ para a estrela Be 28 CMa. Observações visuais (triângulos cinzas) são mostrados em comparação com a modelagem utilizando diferentes valores de $\alpha$, como indicado na figura. Também é apresentado um gráfico de chi-quadrado reduzido para diferentes valores de viscosidade (a linha horizontal pontilhada indica o nível de confiança de 90\%). Créditos: Carciofi et al. (2012).

intercalados. Um resultado importante de Haubois et al. (2012) é que eles mostraram que muitas curvas de luz de estrelas Be eram qualitativamente muito semelhantes às curvas teóricas, o que sugeriu que ao menos parte da variabilidade fotométrica observada em estrelas Be era devida à formação e dissipação de um disco viscoso.

Carciofi et al. (2012) combinaram cálculos hidrodinâmicos e de transferência radiativa para gerar curvas de luz sintéticas e estimar, pela primeira vez na literatura, o valor de $\alpha$ para o disco de uma estrela Be. A estrela modelada foi $28 \mathrm{CMa}$, conhecida por ser uma Be quase-periódica com eventos de disco com duração de 8 anos, aproximadamente (Ghoreyshi et al., 2018). A estimativa do parâmetro de viscosidade foi feita durante a dissipação do disco que se estendeu de 2003 até 2008, o que permitiu a determinação de $\alpha=1,0 \pm 0,2$ (vide painel superior da Fig. 2.1).

Posteriormente, foi compreendido que para modelar a parte dissipativa de um evento de disco é necessário considerar apropriadamente o histórico prévio de injeção de massa no disco (Rímulo et al., 2018). Essa é uma consequência do que se convencionou chamar "efeito de reservatório de massa", que descrevemos em mais detalhes na seção 2.1.1.

O trabalho de Carciofi et al. (2012) foi um importante passo inicial na compreensão das condições físicas do disco de $28 \mathrm{CMa}$ e se tornou a motivação do principal projeto de doutorado do aluno do IAG M. Ghoreyshi. Em um artigo recentemente publicado, 
Ghoreyshi et al. (2018) mostram um modelo da curva de luz completa de 28 CMa nos últimos 34 anos. Este trabalho traz vários avanços com relação ao estudo inicial de Carciofi et al. (2012), que podem ser sumarizados pelos seguintes pontos:

- Pela primeira vez modelou-se eventos de formação e dissipação de disco, mostrando que o VDD é capaz de explicar bem ambas as fases;

- Verificou-se que $\alpha$ é variável no tempo;

- A inclusão do efeito de reservatório de massa levou a uma revisão do $\alpha$ da dissipação iniciada em 2003 para 0,21 $\pm 0,05$ (ver abaixo).

Recentemente, Rímulo et al. (2018) estimaram os parâmetros estelares e de disco para 54 estrelas Be da Pequena Nuvem de Magalhães, SMC, totalizando 81 eventos de disco. Nesse trabalho, os autores demonstraram ser possível utilizar uma pipeline semiautomatizada para a modelagem desses eventos.

No presente trabalho, a metodologia de ajuste desenvolvida por Rímulo et al. foi aplicada para a modelagem de estrelas candidatas a Be da Grande Nuvem de Magalhães, LMC. Na próxima seção, 2.2, apresentamos os conceitos teóricos básicos sobre o VDD dinâmico que embasam a metodologia utilizada, que é descrita na seção 2.3. O leitor interessado, pode encontrar maiores detalhes, como a dedução de certas expressões, por exemplo, na tese Rímulo (2017) e em Rímulo et al. (2018).

\subsubsection{O efeito de reservatório de massa}

Durante o seu doutorado, Rímulo (2017) descobriu um novo efeito, que foi cunhado como "efeito de reservatório de massa". A seguir explicamos, de forma sucinta, a causa e consequências desse efeito. O leitor interessado em maiores detalhes pode consultar a seção 3.3 de Rímulo 2017, além de Rímulo et al. 2018 e Ghoreyshi et al. (2018).

A densidade superficial de um disco viscoso não decorre unicamente da taxa de injeção de massa em um instante de tempo $t$, mas também depende do histórico de injeção de massa precedente a esse instante $t$. Dessa forma, a modelagem da fase dissipativa não pode ser realizada sem considerar a etapa precedente de construção do disco.

Discos que possuem uma fase construtiva mais longa necessariamente formam um maior reservatório de massa e momento angular em suas regiões mais externas, que estão além 


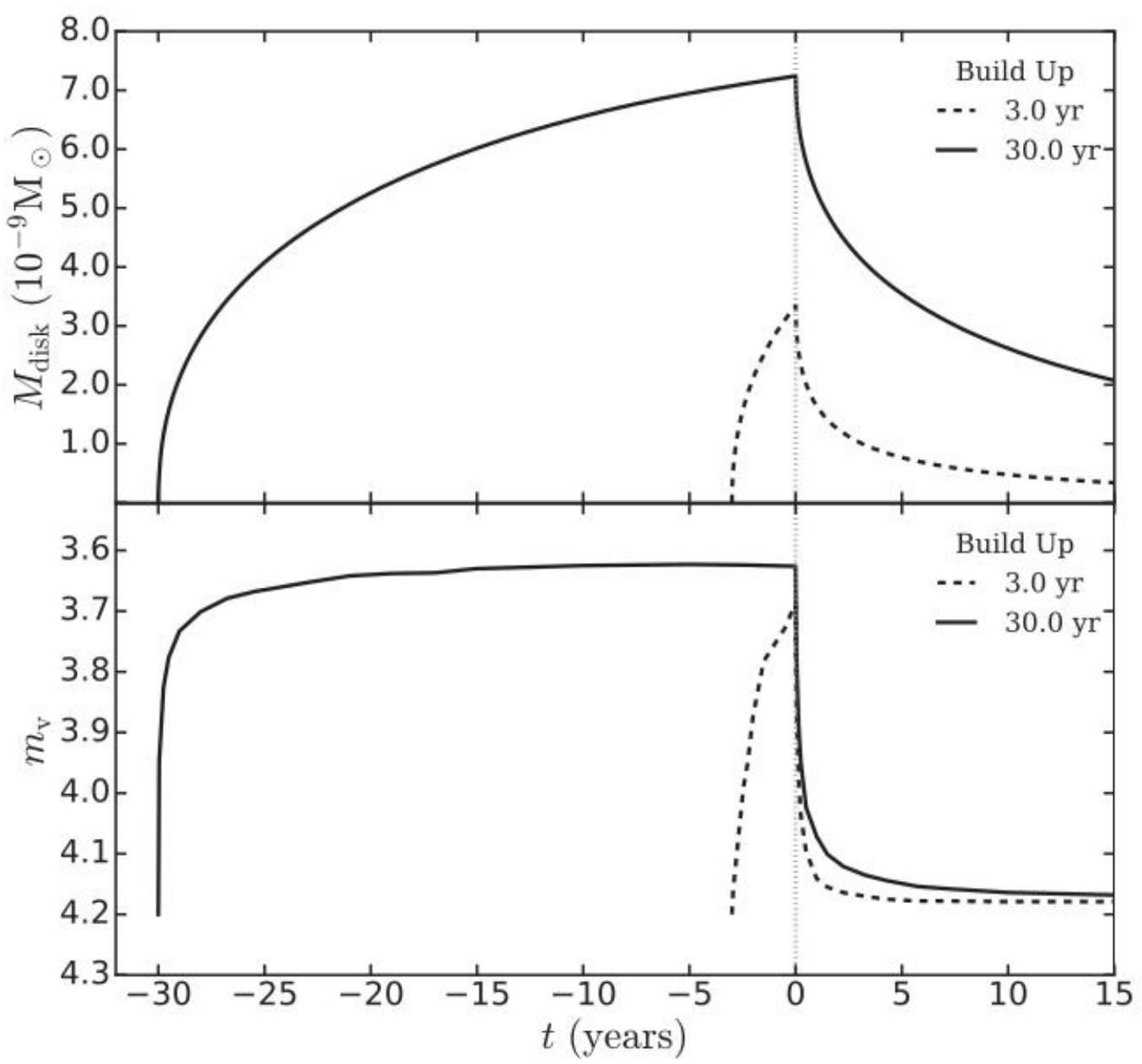

Figura 2.2: Comparação entre dois eventos de formação e dissipação de disco. A linha sólida representa um disco que é alimentado por uma taxa constante por 30 anos. Enquanto o outro modelo (linha pontilhada) tem uma tempo de construção bem mais curto (3 anos). Os autores assumiram $t=0$ para o início da dissipação do disco para ambos modelos. Superior: Massa total do disco em função do tempo. Inferior: Curvas de luz correspondentes. Adaptado de Ghoreyshi et al. (2018).

da faixa de poucos raios estelares onde a fotometria dos observáveis no visível se formam, vide seção 1.3.3. Portanto, o reservatório de massa não é detectável por observações no visível.

Quando a fase dissipativa se inicia (no momento em que a injeção de massa cessa), ocorre a re-acreção de matéria e, se o disco externo é mais massivo em função de um tempo de construção mais prolongado, o disco interno se mantém relativamente denso por maior tempo. Nesse caso, a curva de luz observada possui uma dissipação mais lenta. Da mesma forma, se um disco possui uma fase de construção mais curta, a fase dissipativa é mais rápida pelo fato do reservatório de massa ser menor. Isto é graficamente mostrado na figura 3 de Ghoreyshi et al. (2018), reproduzida na figura 2.2. 
A vantagem de estudar bumps isolados, que se iniciam após uma fase clara de inatividade, é que não há disco presente quando a injeção de massa começa, portanto, não há necessidade de considerar o histórico de perda de massa precedente. A reavaliação da estimativa do valor de $\alpha$ para a fase dissipativa de 28 CMa iniciada em 2003 é um exemplo da importância do efeito de reservatório de massa. Carciofi et al. (2012), modelaram uma fase dissipativa assumindo que uma construção muito longa a antecedeu. Assim, um valor de $\alpha=1,0 \pm 0,2$ foi necessário para reproduzir a dissipação observada. Ghoreyshi et al. (2018) demonstraram que quando a fase de construção do disco é levada em conta de maneira correta, o valor necessário para reproduzir a mesma fase dissipativa é menor, $\alpha=0,21 \pm 0,05$. O leitor interessado pode consultar a seção 5.3 de Ghoreyshi et al. (2018) para mais detalhes.

\subsubsection{Bumps e Dips}

As curvas de luz de estrelas Be apresentam, geralmente, variações em várias escalas de tempo e amplitude. A maioria dessas variações são irregulares, mas, uma fração dessas curvas de luz exibem fenômenos na forma de bumps: um aumento súbito de brilho seguido de um desvanecer frequentemente mais lento. Por vezes, as curvas de luz não apresentam bumps, mas dips: uma súbita diminuição de brilho, seguida de uma recuperação lenta (veja Fig. 2.3).

Os bumps ${ }^{1}$ observados se assemelham às curvas fotométricas sintéticas obtidas por Haubois et al. (2012). Nesse caso específico, os autores estudaram um disco circunstelar que era construído com uma taxa de injeção de massa constante, seguido pela dissipação, quando a injeção de massa era interrompida. Foi demonstrado que os cenários de bumps estão associados à formação e dissipação de disco de estrelas vistas próximas à configuração pole-on (com ângulos de inclinação $i \leq 70^{\circ}$ ), enquanto que os dips estão vinculados a estrelas vistas próxima à configuração edge-on (também conhecidas como estrelas shell, $i \geq 70^{\circ}$ ). O efeito fotométrico da presença do disco é dependente do ângulo de inclinação: no caso edge-on, o disco é visto projetado contra a estrela, tendo o efeito líquido de remover radiação estelar; enquanto que na configuração pole-on o resultado final é um aumento da magnitude, devido ao reprocessamento do fluxo estelar pelo disco (emissão livre-livre e

\footnotetext{
${ }^{1}$ Terminologia: a partir desse ponto, a palavra bumps também será empregada para o coletivo de bump e dip.
} 

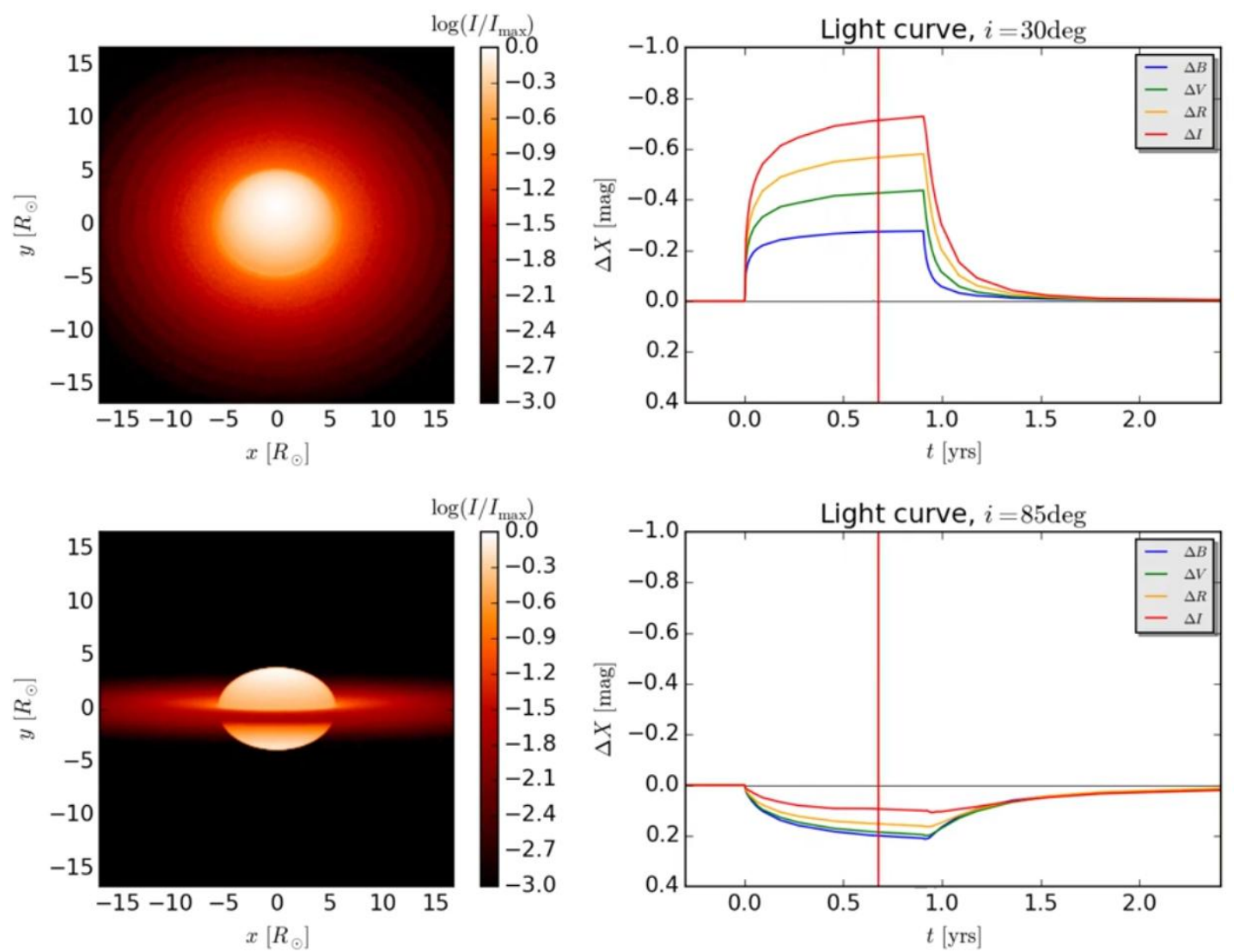

Figura 2.3: Previsão efeito da inclinação nas curvas fotométricas de uma estrela Be, durante evento de formação e dissipação de disco. Na parte superior, apresentamos como a estrela seria vista por um observador cujo ângulo de inclinação é de $i=30^{\circ}$ (à esquerda). Na coluna da direita, as respectivas curvas de luz para as bandas fotométricas $B$ (azul), $V$ (verde), $R$ (amarela) e $I$ (vermelha) são apresentadas. Na parte inferior apresentamos os mesmos resultados para um observador a $i=85^{\circ}$. A linha vertical vermelha, nas duas figuras da coluna direita, representa o tempo (em anos) que se passou desde que a estrela iniciou sua perda de massa. Para esse exemplo, foram utilizadas simulações hidrodinâmicas combinadas com cálculos de transporte radiativo.

livre-ligado). Ambos os casos são ilustrados na Fig. 2.3.

\subsubsection{Modelagem de uma amostra de estrelas Be: uma breve revisão}

Rímulo et al. (2018) modelaram uma amostra de curvas de luz de estrelas da SMC que possuem bumps e dips, obtendo parâmetros estelares e do disco, além de estimarem os valores da taxa de perda de massa e momento angular. A amostra é constituída por 54 estrelas, totalizando 81 eventos de disco. A modelagem foi feita utilizando uma lista de procedimentos (uma pipeline) desenvolvida pelos autores. Uma grade de curvas de luz sintéticas, obtidas com simulações hidrodinâmicas unidas a cálculos de transferência radiativa, foi utilizada para a modelagem desses eventos. A comparação entre as curvas de 
luz sintéticas e observadas foi possível através de expressões analíticas desenvolvidas pelos autores que reproduzem, aproximadamente, o comportamento fotométrico observado para esses eventos.

O trabalho de Rímulo et al. (2018) traz contribuições importantes para os estudos dinâmicos de estrelas Be. Com esse estudo, o número de estrelas cujo valor da viscosidade, $\alpha$, é conhecido foi multiplicado 54 vezes. É a primeira determinação, estatisticamente significativa, de viscosidade para estrelas Be. Os valores obtidos para $\alpha$ são da ordem de alguns décimos, semelhantes aos valores estimados para os discos de novas anãs (King et al. 2007; Kotko e Lasota 2012). Ao se olhar para a amostra como um todo, a viscosidade durante a fase de construção do disco, $\left\langle\alpha_{b u}\right\rangle=0,63^{2}$, é aproximadamente o dobro do que o valor encontrado para a fase dissipativa, $\left\langle\alpha_{d}\right\rangle=0,29$. As taxas de perda de massa e momento angular típicas para esses eventos são da ordem de $\sim 10^{-10} M_{\odot}$ ano $^{-1}$ e $\sim 5 \times$ $10^{36} \mathrm{~g} \mathrm{~cm}^{2} \mathrm{~s}^{-2}$, respectivamente. Mesmo para os discos mais massivos, a perda de momento angular da estrela é menor que os valores previstos pelos melhores modelos de evolução estelar para que as estrelas não atinjam velocidades críticas. Essas estimativas oferecem, pela primeira vez, limitações para o mecanismo de transporte de momento angular interno para estrelas massivas em altas rotações.

As extensões naturais desse estudo abrangem amostras de curvas de luz de estrelas Be da LMC e da Galáxia. Essa dissertação é principalmente constituída pelo estudo das curvas de luz de estrelas candidatas a Be da LMC.

\subsection{Principais considerações teóricas}

Para a elaboração do texto a seguir, utilizamos como principal referência Rímulo (2017). Além da tese de Rímulo, análises mais detalhas e abordagens diferentes para o problema podem ser encontradas em Lee et al. (1991), Okazaki (2001), Bjorkman e Carciofi (2005), Okazaki (2007), Jones et al. (2008), Carciofi e Bjorkman (2008) e Krtička et al. (2011).

As próximas seções objetivam apresentar os principais conceitos e parâmetros envolvidos na modelagem utilizada neste trabalho. As expressões são apresentadas em coordenadas cilíndricas $(R, \phi, z)$.

No contexto dinâmico, a evolução da densidade superficial de um disco de estrela Be

\footnotetext{
${ }^{2} \mathrm{O}$ subscrito up vem do inglês, Build up, que significa construção.
} 
geometricamente fino e com simetria axial é descrito pela equação (Papaloizou e Lin, 1995)

$$
\frac{\partial \Sigma}{\partial t}+\frac{1}{R} \frac{\partial}{\partial R}\left(R \Sigma v_{\mathrm{R}}\right)=S_{\Sigma}
$$

onde a quantidade $v_{\mathrm{R}}$ é a componente radial da velocidade do disco e $\Sigma$ é a sua densidade superficial, que pode ser definida como

$$
\Sigma(R, t)=\int_{-\infty}^{+\infty} \rho(R, z, t) \mathrm{d} z=\int_{-\infty}^{+\infty} \rho(R, 0, t) e^{\left[-\frac{z^{2}}{2 H^{2}}\right]} \mathrm{d} z=\sqrt{2 \pi} H \rho_{0}(R, t),
$$

no qual $\rho$ é a densidade volumétrica e $\rho_{0}$ é a densidade de base no plano médio $(z=0)$ do disco, e a escala de altura, pode ser expressa como

$$
H(R)=R\left(c_{\mathrm{s}} / v_{\phi}\right) \approx R\left(c_{\mathrm{s}} / v_{\mathrm{k}}\right)
$$

sendo que $c_{\mathrm{s}}$ é a velocidade do som dada por

$$
c_{\mathrm{s}}^{2}=\frac{k T_{\text {disco }}}{\mu m_{\mathrm{H}}}
$$

e $v_{\phi}$ é a componente azimutal da velocidade do disco, que foi aproximada para uma rotação Kepleriana, com $v_{\mathrm{K}}=v_{\text {orb }} R^{-1 / 2}$. Nas expressões 2.2 está implicitamente assumido que o disco tem uma temperatura constante (isotérmica). Esta simplificação resulta em um perfil de densidade vertical Gaussiano (Carciofi, 2011).

Estudos posteriores fizeram uma análise mais completa do regime de rotação do disco. Krtička et al. (2011) calculou, a partir das equações de fluído, a componente azimutal do campo de velocidade, e demonstrou que na parte mais interna do disco o campo de velocidade é realmente muito próximo de um campo Kepleriano, como pode ser visto na Fig. 2.4. Essa aproximação para a velocidade é válida até $\sim 100 R_{\star}$. Ou seja, a aproximação Kepleriana não produz efeito relevante observacionalmente (vide que a região de formação da banda fotométrica $V$, por exemplo, ocorre para $R \lesssim 2 R_{\star}$, para o caso mais denso, da Fig. 1.6).

A grandeza $R \Sigma v_{R}$ na Eq. 2.1 é o fluxo de massa no disco por ângulo azimutal e $S_{\Sigma}$ representa as fontes e sorvedouros de massa no disco por unidade de área. Fazendo a hipótese de que o principal mecanismo que controla a dinâmica do disco é a viscosidade, podemos expressar o fluxo de massa no disco como

$$
2 \pi R \Sigma v_{\mathrm{R}}=-4 \pi\left(\frac{R^{3}}{G M}\right)^{1 / 2} \frac{1}{R} \frac{\partial}{\partial R}\left(R^{2} \Sigma \alpha c_{\mathrm{s}}^{2}\right)
$$




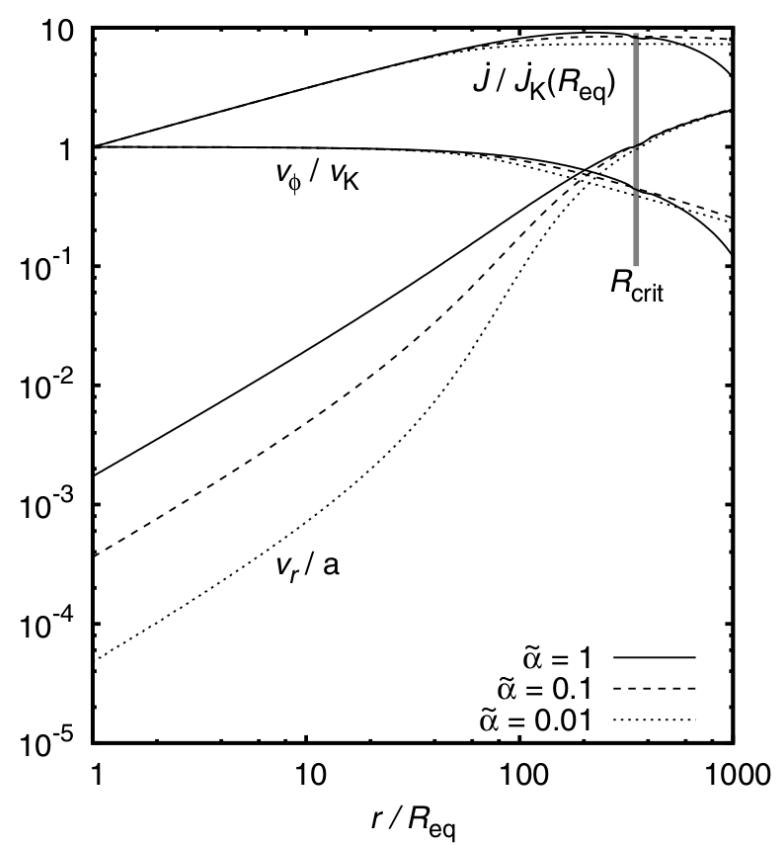

Figura 2.4: Dependência das velocidades radial, azimutal e da taxa de perda de momento angular (em unidades da taxa de perda de momento angular do equador $\dot{J}_{\mathrm{K}}\left(R_{\text {eq }}\right)$. Modelos para um disco isotérmico $\left(T_{0}=\frac{1}{2} T_{\text {ef }}\right)$ para diferentes valores de viscosidade $\tilde{\alpha}$ mostrados na figura. Fonte: Krtička et al. 2011.

sendo que $\alpha$ é o parâmetro de viscosidade. Rímulo et al. (2018) assume que $\alpha$ é constante em $R$, mas permite que esse valor varie com o tempo, como evidências recentes apontam (Ghoreyshi et al., 2018).

Para estimar a temperatura do disco, utilizamos $T_{\text {disco }}=0,6 T_{\text {ef }}$ (Carciofi e Bjorkman, 2006), onde $T_{\text {ef }}$ é a temperatura efetiva estelar.

É possível reescrever a Eq. 2.1, utilizando a expressão 2.5, obtendo

$$
\frac{\partial \Sigma}{\partial t}=\frac{1}{\tau}\left\{\frac{2}{R} \frac{\partial}{\partial R}\left[R^{1 / 2} \frac{\partial}{\partial R}\left(R^{2} \Sigma\right)\right]+\tau S_{\Sigma}\right\}
$$

onde introduzimos o parâmetro de escala de tempo, $\tau(t)$, dado por

$$
\tau(t)=\frac{1}{\alpha(t)}\left(\frac{R_{\mathrm{eq}}^{3}}{G M}\right)^{1 / 2} \frac{v_{\mathrm{orb}}^{2}}{c_{\mathrm{s}}^{2}} .
$$

Como definido na Eq. 2.7, o parâmetro de escala de tempo é proporcional à escala de tempo viscosa no equador estelar, que é dada por $T_{\text {visc }}=3 R v_{\mathrm{k}} / 2 \alpha c_{\mathrm{s}}^{2}$.

A expressão 2.6 descreve quão rapidamente a matéria é difundida através do disco. A vantagem de introduzir $\tau(t)$ é a separação da dependência temporal de $\alpha(t)$ do problema de solucionar a Eq. 2.6. Assim, ao modelar a curva de luz de uma estrela Be podemos estimar o valor de $\tau(t)$ e, desde que saibamos os valores de $R_{\text {eq }}, T_{\text {disco }}$ e $M$ é possível determinar o valor de $\alpha$ (como, por exemplo, Carciofi et al. 2012). 
Para que possamos gerar modelos que não sejam dependentes do tempo físico $t$ e dos demais parâmetros estelares $\left(R_{\mathrm{eq}}, T_{\mathrm{disco}}, M\right)$, introduzimos um parâmetro de tempo adimensional

$$
\mathrm{d} \tilde{\tau}=\frac{\mathrm{d} t}{\tau(t)}
$$

Assim, podemos construir uma grade de soluções para a Eq. 2.6 que é independente de $\alpha(t), R_{\text {eq }}, T_{\text {disco }}$ e $M$.

\subsubsection{Injeção e perda de massa no disco}

O termo $S_{\Sigma}$ na Eq. 2.6 representa a troca de massa entre disco-estrela e disco-meio externo (através do raio externo do disco). Quando a estrela está injetando massa no disco, consideramos que esta é colocada em órbita próximo ao equador estelar a partir de um anel de largura desprezível e raio $R_{\text {inj }}$. Consequentemente, podemos expressar a taxa de injeção de massa por unidade de área como $\dot{M}_{\text {inj }}(t) \delta R / 2 \pi R$, onde $M_{\text {inj }}$ é a taxa de massa injetada. O disco também pode perder massa através dos seus limites interno $\left(R_{\mathrm{inj}}\right)$, caindo de volta na estrela, e externo $\left(R_{\text {ext }}\right)$ quando a matéria abandona o sistema. Dessa forma, a função fonte pode ser expressa como

$$
S_{\Sigma}=\dot{M}_{\text {inj }}(t) \Upsilon\left(R_{\text {inj }}\right)+\dot{M}_{\text {eq }}(t) \Upsilon\left(R_{\text {eq }}\right)+\dot{M}_{\text {ext }}(t) \Upsilon\left(R_{\text {ext }}\right)
$$

onde utilizamos $\Upsilon\left(R^{\prime}\right)=\delta\left(R-R^{\prime}\right) / 2 \pi R$ e $\dot{M}_{\text {eq }}$ é a taxa de massa removida no equador estelar (note que $\dot{M}_{\text {eq }} \leq 0$ ), sendo dada por

$$
\dot{M}_{\mathrm{eq}}(t)=-\left.4 \pi\left(\frac{R_{\mathrm{eq}}}{G M}\right)^{1 / 2} \frac{\partial}{\partial R}\left(\alpha c_{\mathrm{s}}^{2} R^{2} \Sigma\right)\right|_{R_{\mathrm{eq}}^{+}} .
$$

De forma semelhante, $\dot{M}_{\text {ext }}(t)$ é a massa perdida através do raio externo $R_{\text {ext }}$, sendo dada por

$$
\dot{M}_{\text {ext }}(t)=-\left.4 \pi\left(\frac{R_{\mathrm{ext}}}{G M}\right)^{1 / 2} \frac{\partial}{\partial R}\left(\alpha c_{\mathrm{s}}^{2} R^{2} \Sigma\right)\right|_{R_{\mathrm{ext}}^{-}} .
$$

A solução para o estado estacionário de um disco viscoso corresponde ao caso limite de um disco que é alimentado por uma taxa constante por um tempo infinitamente grande. Para obtê-lo, fazemos $\partial \Sigma / \partial t=0$ na Eq. 2.6 (ou 2.1) e assumimos que o parâmetro de viscosidade, $\alpha$, e a taxa de injeção de massa, $M_{\text {inj }}$, não dependem do tempo.

A expressão 2.9 aponta que o disco pode ser dividido em duas partes, uma parte mais interna localizada entre o raio de injeção de massa no disco e a estrela $\left(1 \leq \tilde{R} \leq \tilde{R}_{\text {inj }}\right)$, 
e outra região muito mais extensa que abrange desde o raio de injeção até o raio externo $\left(\tilde{R}_{\text {inj }} \leq \tilde{R} \leq \tilde{R}_{\text {ext }}\right)$, onde utilizamos $\tilde{R}=R / R_{\text {eq }}, \tilde{R}_{\text {inj }}=R_{\text {inj }} / R_{\text {eq }}$ e $\tilde{R}_{\text {ext }}=R_{\text {ext }} / R_{\text {eq }}$.

Impondo $\partial \Sigma / \partial t=0$ à equação 2.1 , o disco estacionário será governado pela solução da equação

$$
\frac{\partial}{\partial R}\left(2 \pi R \Sigma v_{\mathrm{R}}\right)=\dot{M}_{\mathrm{inj}} \delta\left(R-R_{\mathrm{inj}}\right), 1 \leq \tilde{R} \leq \tilde{R}_{\mathrm{ext}},
$$

onde utilizamos a definição de função fonte da Eq. 2.9.

A solução ${ }^{3}$ da Eq. 2.12 é dada por

$$
\Sigma_{\text {est }}(\tilde{R})=\left\{\begin{array}{ll}
\frac{\Sigma_{0}}{\tilde{R}^{2}} \Xi\left(\frac{\tilde{R}^{\frac{1}{2}}-1}{\tilde{R}_{\text {inj }}^{\frac{1}{2}}-1}\right), & 1 \leq \tilde{R}<\tilde{R}_{\mathrm{inj}} \\
\frac{\Sigma_{0}}{\tilde{R}^{2}}\left(\frac{\tilde{R}_{\text {ext }}^{\frac{1}{2}}-\tilde{R}^{\frac{1}{2}}}{\tilde{R}_{\mathrm{ext}}^{\frac{1}{2}}-1}\right), & \tilde{R}_{\mathrm{inj}} \leq \tilde{R}<\tilde{R}_{\mathrm{ext}}
\end{array},\right.
$$

onde a densidade superficial em $\tilde{R}=1$ pode ser escrita como

$$
\Sigma_{0}=\frac{1}{\Xi} \tilde{R}_{\mathrm{inj}}^{2} \Sigma_{\mathrm{inj}}
$$

Seguindo a notação de Rímulo, vamos nos referir a essa quantidade como a densidade superficial assintótica. O valor assintótico só pode ser atingido depois que o disco é alimentado por uma $M_{\text {inj }}$ constante por um tempo infinitamente longo. O número $\Xi$ varia entre 0 e 1, e representa a fração da massa injetada que cai de volta na estrela. Esse valor pode ser expresso como

$$
\Xi=\frac{R_{\mathrm{ext}}^{2}-R_{\mathrm{inj}}^{2}}{R_{\mathrm{ext}}^{2}-R_{\mathrm{eq}}^{2}}
$$

Uma vez que $R_{\text {inj }}$ é bem próximo de $R_{\text {eq }}$ e $R_{\text {ext }}$ é bem maior que este valor, $\Xi$ é um número pouco menor que a unidade para qualquer estrela Be. Como os valores de $\tilde{R}_{\text {inj }}$ e $\tilde{R}_{\text {ext }}$ são

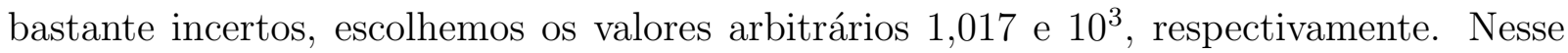
caso, $1-\Xi=2,84 \times 10^{8}$.

É possível relacionar $\Sigma_{0}$ com $\dot{M}_{\text {inj }}$ da seguinte forma

$$
2 \pi R_{\mathrm{eq}} \Sigma_{0}\left(\frac{R_{\mathrm{eq}}}{\tau}\right)=\dot{M}_{\mathrm{inj}}\left(\tilde{R}_{\mathrm{inj}}^{\frac{1}{2}}-1\right) \equiv\left(-\frac{\partial M}{\partial t}\right)_{\mathrm{tip}},
$$

onde denotamos a taxa de perda de massa típica por $(-\partial M / \partial t)_{\text {tip }}$.

Esta equação nos mostra a taxa de decréscimo viscoso típica é muito menor que a taxa de injeção de massa, $M_{\text {inj }}(\tilde{\tau})$. Isso quer dizer que a maioria da massa injetada cai novamente na estrela e apenas uma pequena fração da massa injetada é responsável pelo

\footnotetext{
${ }^{3}$ A derivação detalhada dessa solução pode ser encontrada na seção 3.2 de Rímulo (2017).
} 
crescimento do disco. Este resultado foi obtido pela primeira a partir de simulações $\mathrm{SPH}^{4}$ de discos de Be por Okazaki et al. (2002). Os autores identificaram que apenas algo em torno de $0,1 \%$ do material injetado entra em órbita.

A quantidade de matéria que o disco está perdendo através de $R_{\text {ext }}$ é de $\dot{M}_{\text {inj }}(1-\Xi)$. Uma vez que a massa de um disco estacionário não se altera, $\dot{M}_{\text {inj }}(1-\Xi)$ é a massa perdida pela estrela, que indicaremos por $(-\partial M / \partial t)_{\text {est }}$. Esse valor pode ser relacionado com a taxa de decréscimo típica por

$$
\left(-\frac{\partial M}{\partial t}\right)_{\mathrm{est}}=\frac{\Lambda}{\tilde{R}_{\mathrm{ext}}^{\frac{1}{2}}}\left(-\frac{\partial M}{\partial t}\right)_{\mathrm{tip}},
$$

onde $(-\partial M / \partial t)_{\text {tip }}$ é a taxa de perda de massa típica e $\Lambda=1 /\left(1-\tilde{R}_{\text {ext }}^{-1 / 2}\right)$ é um número próximo de 1 para discos de Be em geral. Em nosso caso específico, $\Lambda-1=3,27 \times 10^{-2}$, aproximadamente da mesma ordem de grandeza que o valor obtido por Okazaki et al. (2002) reportado logo acima.

No caso estacionário o momento angular perdido pelo sistema (e consequentemente pela estrela) é $\left(G M R_{\text {eq }}\right)^{\frac{1}{2}} \dot{M}_{\text {inj }}(1-\Xi)$, sendo dado por

$$
\left(-\frac{\partial J}{\partial t}\right)_{\mathrm{est}}=\Lambda\left(G M R_{\mathrm{eq}}\right)^{\frac{1}{2}}\left(-\frac{\partial M}{\partial t}\right)_{\mathrm{tip}} .
$$

Há uma diferença importante entre as equações das taxas de perda de massa e momento angular. Na Eq. 2.17 vemos que é necessário conhecermos $R_{\text {ext }}$ para estimarmos a taxa de perda de massa. Como dito acima, este parâmetro é difícil de ser obtido para estrelas Be (ver discussão a respeito em Klement et al. 2017). Uma vez que para calcularmos a taxa de momento angular perdido precisamos apenas de $R_{\text {eq }}$, a determinação dessa quantidade é mais confiável que a taxa de perda de massa.

\subsection{Uma grade de curvas de luz sintéticas}

Como discutido na seção 2.2, a vantagem de introduzir o parâmetro $\tilde{\tau}$, é que podemos construir uma grade de modelos que são solução da Eq. 2.1 de forma independe do tempo físico $t$. Pois do contrário, a alternativa seria a modelagem individual de cada evento de disco. Adicionalmente, nossos modelos são independentes dos parâmetros $M, T_{\text {ef }}, R_{\text {eq }}$ e $\alpha(t)$ da estrela em consideração. Esses parâmetros definem o parâmetro de escala de tempo $\tau(t)$ (veja Eq. 2.7), e também acopla o tempo físico $t$ com o parâmetro de tempo $\tilde{\tau}$ (Eq. 2.8).

\footnotetext{
${ }^{4}$ Smoothed-Particle Hydrodynamics.
} 
De forma sucinta, os modelos são compostos por uma estrela inicialmente sem disco que começa a perder massa em $\tilde{\tau}=0$. A perda de massa ocorre até $\tilde{\tau}=\tilde{\tau}_{b u}$, quando o disco começa a dissipar.

Foram escolhidos 11 valores de $\tilde{\tau}_{\text {bu }}$ que estão listados na tabela 2.1. Esses valores correspondem à tempos de construção de, no mínimo 15 dias (d), se considerarmos estrelas Be recentes na SP com $\alpha \lesssim 1$ e que o parâmetro de escala de tempo (Eq. 2.7) é da ordem de $\sim(100-200) / \alpha$ d. Optou-se por não modelar eventos mais curtos que 15 d, que são classificados como flickers por Keller et al. (2002).

Agora, passamos à descrição da grade de modelos empregadas nesse estudo. Ressaltamos que a grade utilizada no presente estudo é a computada para estrelas da SMC utilizada por Rímulo et al. (2018). Uma grade especificamente voltada à estrelas da LMC está atualmente sendo computada em colaboração com L. Rímulo, o que permitirá refinar os resultados deste trabalho futuramente.

\subsubsection{O código SINGLEBE: simulações dinâmica de discos}

A Eq. 2.1 pode ser multiplicada por $R^{2}$ e ser reescrita como

$$
\frac{\partial R^{2} \Sigma}{\partial t}=2 \frac{\partial}{\partial \ln R}\left[\left(\frac{1}{G M R}\right)^{\frac{1}{2}} \frac{\partial}{\partial \ln R}\left(\alpha c_{\mathrm{s}}^{2} R^{2} \Sigma\right)\right]+R^{2} S_{\Sigma}
$$

A expressão 2.19 é uma equação diferencial parcial solucionada pelo código SINGLEBE (Okazaki, 2007). A tarefa do código é simular a evolução temporal de um disco viscoso com simetria axial. A Eq. 2.19 é resolvida utilizando o esquema FTCS ${ }^{5}$.

A grade radial de células utilizada é logarítmica, definida por

$$
\ln \tilde{R}[i]=\ln \tilde{R}_{1}+\left(i-i_{1}\right) \frac{\ln \tilde{R}_{2}-\ln \tilde{R}_{1}}{i_{21}}, \text { para } i=1,2,3, \ldots, N
$$

onde $\tilde{R} \equiv R / R_{\mathrm{eq}}$.

Nessa definição, o usuário escolhe dois raios $\tilde{R}_{1}$ e $\tilde{R}_{2}$ e os seus respectivos índices na grade dados por $i_{1}$ e $i_{1}+i_{21}$, respectivamente. Ou seja, o usuário define $\tilde{R}_{1}=\tilde{R}\left[i_{1}\right]^{6}$ e $\tilde{R}_{2}=\tilde{R}\left[i_{1}+i_{21}\right]$, além da extensão da grade depois de $R_{2}$, através do número $n_{2}$. Dessa forma, o número total de células é de $N=i_{1}+i_{21}+n_{2}$.

Em Ghoreyshi et al. (2018), Rímulo et al. (2018) e nesse trabalho, a grade radial é dada por $\tilde{R}_{1}[1]=1$ e $\tilde{R}_{2}[400]=1000$, definindo o raio de injeção de massa como $\tilde{R}[2]=1,017$.

\footnotetext{
${ }^{5}$ Acrônimo de Forward-Time Central-Space.

${ }^{6}$ Caso $i_{1}>1$, haverá $i_{1}-1$ raios antes de $R_{1}$.
} 

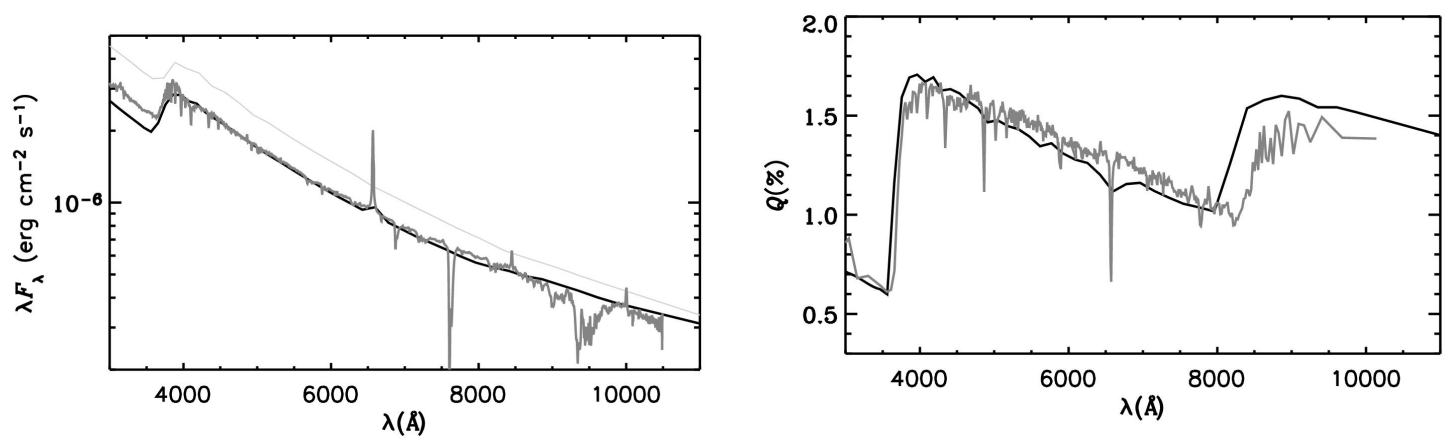

Figura 2.5: Esquerda: Média do espectro emergente no visível para a estrela $\zeta$ Tauri e média da polarização no contínuo, na direita. As médias são calculadas utilizando dados de Wood et al. (1997). Os dados observacionais são mostrados em cinza e em preto estão representados os resultados dos modelos 2D obtidos por Carciofi et al. (2009). Adaptado de Carciofi et al. (2009).

Assim, o limite interno da grade radial está no equador estelar e a injeção de massa no disco ocorre a uma distância um pouco além do equador.

\subsubsection{Cálculo do transporte de energia com HDUST}

A principal das ferramentas desenvolvidas pelo grupo de estudo de estrelas Be do IAGUSP é o código HDUST. O código vem sendo empregado com sucesso na modelagem de várias estrelas Be e B[e] (Carciofi et al. 2006, 2007, 2008, 2009, 2010, 2012; Klement et al. 2015, 2017, entre outros trabalhos).

Sumariamente, a tarefa do código é converter as configurações estruturais dadas pelo usuário em observáveis astrofísicos. Entre outros, o HDUST calcula observáveis como o espectro polarizado, distribuição de energia espectral, perfis de linha, imagens e mapas de emissão (veja Fig. 2.5). O código emprega o método de Monte Carlo para resolver o transporte radiativo da luz polarizada no meio circunstelar. A solução depende das opacidades em cada célula, cujos cálculos dependem das estruturas de excitação, ionização, temperatura e densidade do disco. A priori, nenhuma dessas grandezas é conhecida, e o HDUST adota um método iterativo para um cálculo auto-consistente, embora seja possível manter algumas quantidades fixas (como a densidade de base do disco, perfis de densidade entre outros). Outra possibilidade que o código oferece é a leitura de um perfil de densidade, como o gerado pelo SINGLEBE, seguido pelo cálculo iterativo das quantidades acima mencionadas. 

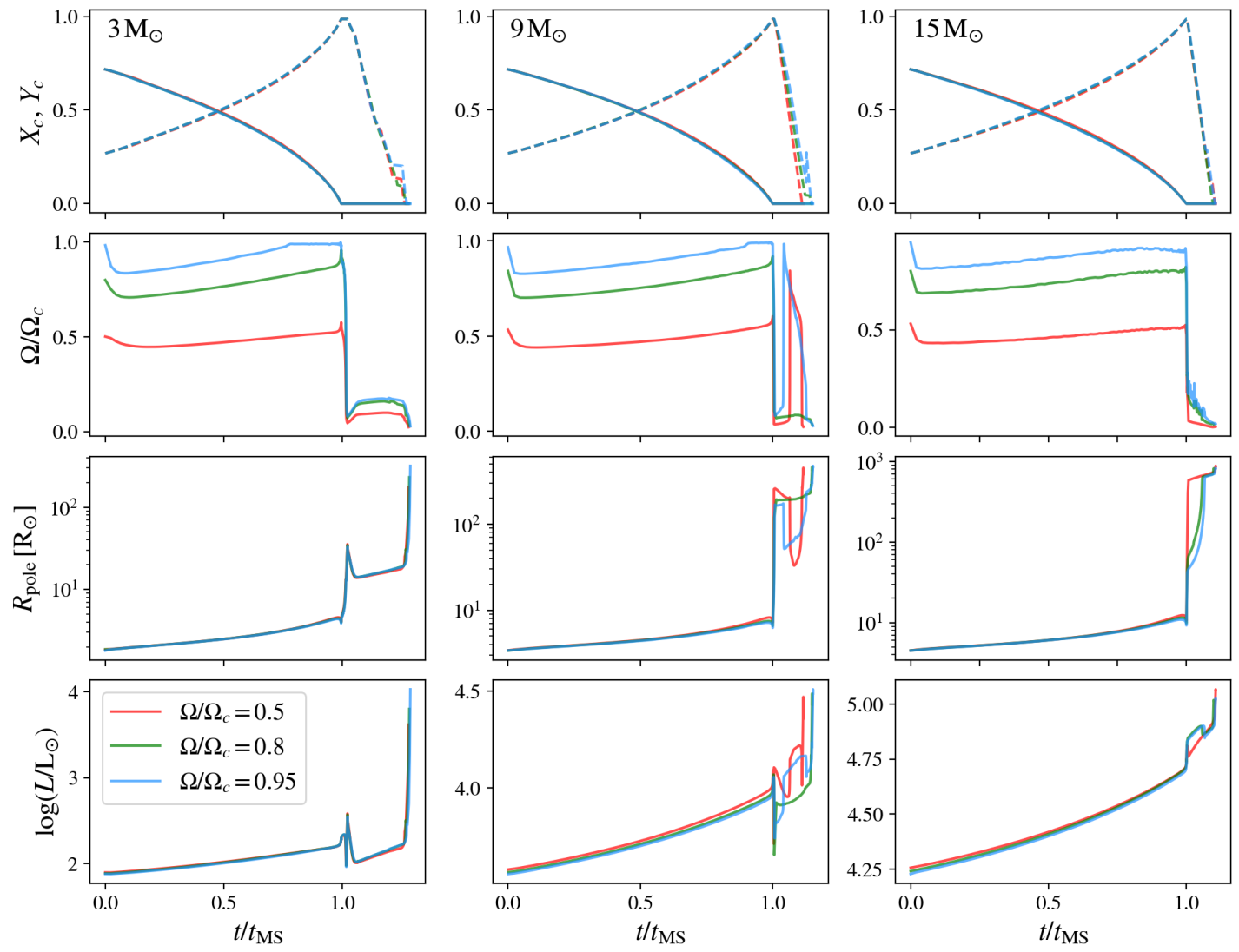

Figura 2.6: Alteração de parâmetros ao longo da evolução para estrelas de 3, 9 e $15 M_{\odot}$, para diferentes taxas de rotação (apresentadas na imagem), fornecidas pelo código de Genebra. A evolução das frações de H e He no núcleo são apresentadas nas imagens da primeira linha. Na linha imediata, vemos a evolução da taxa de rotação estelar, seguido pelos valores de raio do polo e de luminosidade $(L)$. Note que o eixo horizontal das figuras representa a razão entre a idade da estrela e o seu tempo de estadia na sequência principal.

\section{A estrela central}

Os modelos estelares são definidos pelos parâmetros $M, R_{\text {eq }}, T_{\text {ef }}, W$ e $\beta$ (veja Eq. 2.21). Devido à sua alta rotação, as estrelas Be possuem uma forma oblata, além de possuir polos mais quentes que o equador. Podemos expressar a razão entre os raios do equador e polar como $R_{\text {eq }} / R_{\text {polo }}=1+W^{2} / 2$ (veja Eq. 1.5 ), se considerarmos a aproximação de Roche (vide seção 1.3.3).

O código HDUST possui a opção de utilizar uma estrela oblata, cuja temperatura superficial é dependente da latitude, sendo proporcional à gravidade efetiva da seguinte forma

$$
T_{\mathrm{sup}} \propto g_{\mathrm{ef}}^{\beta}
$$




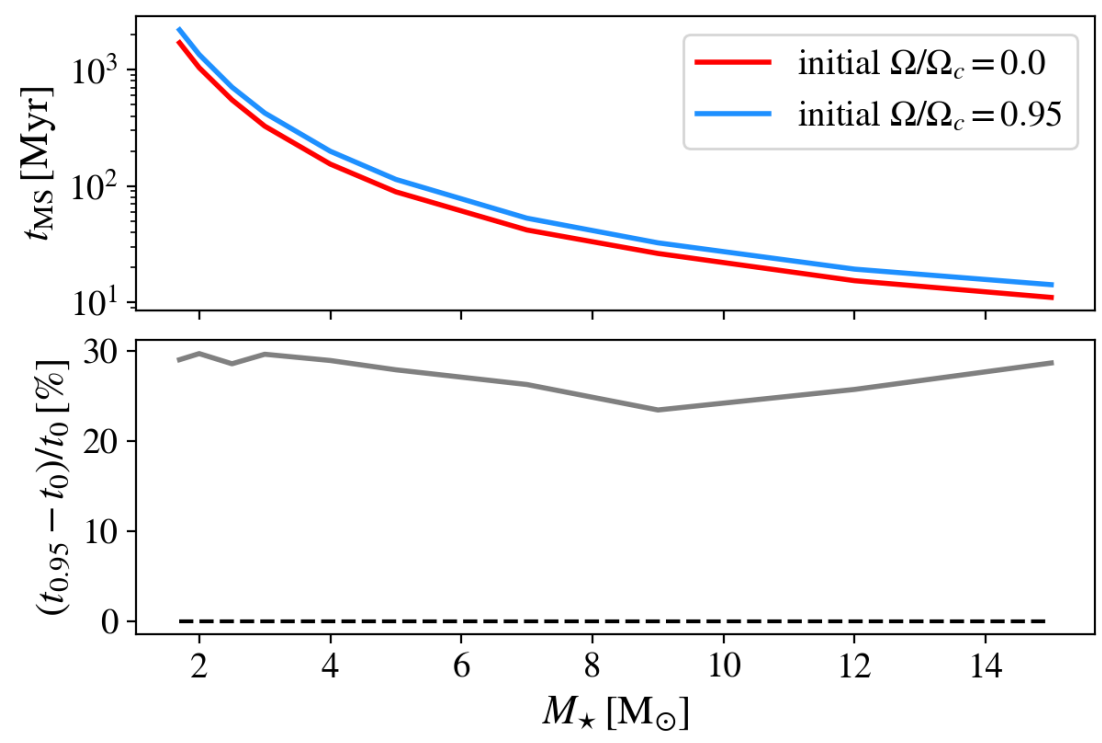

Figura 2.7: Superior: Modificação do tempo de permanência da estrela na SP devido à rotação, como função da massa estelar. Inferior: Aumento percentual da estadia da estrela na SP por efeito de altas taxas de rotação, em função da massa da estrela.

O coeficiente $\beta(W)$ é obtido através do ajuste de uma reta ao gradiente de

$$
\partial \ln T_{\mathrm{ef}} / \partial \ln g_{\mathrm{ef}},
$$

dado pela teoria de fluxo de Espinosa Lara e Rieutord (2011). Para a escala de altura do disco (Eq. 2.3), assumimos um disco isotérmico com temperatura de $T_{\text {disco }}=0,6 T_{\text {ef }}$, onde $T_{\text {ef }}$ é a temperatura efetiva estelar, que é definida por $T_{\text {ef }}=\left(L_{\star}\right)^{1 / 4}\left(\sigma S_{\star}\right)^{-1 / 4}$, onde $S_{\star}$ é a área da superfície estelar e $L_{\star}$ sua luminosidade.

Adicionalmente ao efeito da rotação, os parâmetros estelares também são alterados pela evolução estelar (Fig. 2.6 e 2.7), de modo que estrelas Be podem ser encontradas em classes de luminosidade de V a III (Rivinius et al., 2013). Como referência para os parâmetros físicos da estrela, utilizamos os modelos evolutivos fornecidos pelo código de evolução estelar de Genebra (Georgy et al., 2013). Os parâmetros estelares são obtidos através de uma interpolação dos modelos em metalicidade $(Z)$, massa estelar, rotação e idade $\left(t / t_{\mathrm{MS}}\right.$, onde $t / t_{\mathrm{MS}}$ é um número que varia entre de 0 a 1 para uma estrela na Sequência Principal).

\section{Magnitudes sintéticas}

Para compararmos as curvas de luz sintéticas com as observadas, devemos calcular magnitudes sintéticas à partir do fluxo calculado pelo código HDUST. As magnitudes 
Tabela 2.1 - Parâmetros da grade de modelos de bumps.

\begin{tabular}{crcr}
\hline Estrela & $i\left[^{\circ}\right]$ & $\Sigma\left[\mathrm{g} \mathrm{cm}^{-2}\right]$ & $\tilde{\tau}_{\text {bu }}$ \\
\hline 1 & 0,0 & 0,30 & 0,15 \\
2 & 21,8 & 0,41 & 0,45 \\
3 & 31,0 & 0,56 & 0,75 \\
& 38,2 & 0,75 & 1,50 \\
& 44,4 & 1,01 & 2,25 \\
& 50,0 & 1,37 & 3,00 \\
& 55,2 & 1,85 & 4,50 \\
& 60,0 & 2,50 & 6,00 \\
& 64,6 & & 9,00 \\
& 69,1 & & 15,00 \\
& 73,4 & & 30,00 \\
& 77,6 & & \\
& 81,8 & & \\
& 85,9 & & \\
& 90,0 & &
\end{tabular}

sintéticas são geradas utilizando os filtros fotométricos BVRI de Johnson-Cousins de Bessell (1990).

No sistema fotométrico de Johnson-Cousins, a magnitude em um dado filtro $X$ é comparado com medidas da estrela Vega no mesmo filtro, da seguinte forma

$$
M_{\mathrm{X}}=-\frac{5}{2} \log \left(\frac{\left\langle\lambda F_{\lambda}\right\rangle_{\mathrm{X}}}{\left\langle\lambda F_{\lambda}^{\text {Vega }}\right\rangle_{\mathrm{X}}}\right)+M_{\mathrm{X}}^{\mathrm{Vega}}
$$

onde se define $M_{\mathrm{X}}^{\mathrm{Vega}}=0,03$, para cada um dos filtros. A definição de $\left\langle\lambda F_{\lambda}\right\rangle_{\mathrm{X}}$ é

$$
\left\langle\lambda F_{\lambda}\right\rangle_{\mathrm{X}}=\frac{\int_{0}^{\infty} \lambda F_{\lambda} R_{\mathrm{X}}(\lambda) \mathrm{d} \lambda}{\int_{0}^{\infty} R_{\mathrm{X}}(\lambda) \mathrm{d} \lambda}
$$

onde $R_{\mathrm{X}}(\lambda)$ é a curva de transmissão de um dado filtro fotométrico e $F_{\lambda}(\lambda)$ é a densidade de fluxo, em comprimento de onda, que é fornecida pelo código HDUST.

Parâmetros da grade de modelos

Rímulo et al. (2018) utilizaram as definições da seção 2.2 para construir uma grade de modelos que é solução da Eq. 2.19. A grade é composta por 11 modelos dinâmicos descritos no início da seção 2.3, além de 8 diferentes valores de $\Sigma_{0}, 15$ valores de $\cos i$, 
Tabela 2.2 - Parâmetros estelares dos modelos da Tab. 2.1.

\begin{tabular}{cccccc}
\hline Estrela & $Z$ & $M\left[M_{\odot}\right]$ & $W$ & $t / t_{\mathrm{MS}}$ & $\alpha \tau[d]$ \\
\hline 1 & 0,002 & 7 & 0,81 & 0,5 & 90,4 \\
2 & 0,002 & 11 & 0,81 & 0,5 & 103,3 \\
3 & 0,002 & 15 & 0,81 & 0,5 & 118,9 \\
\hline
\end{tabular}

igualmente espaçados. Esses valores estão representados na tabela 2.1. Todo o processo é realizado para 3 modelos estelares diferentes (veja tabela 2.2), de acordo com os modelos estelares de Georgy et al. (2013) (totalizando $11 \times 8 \times 15 \times 3=3960$ curvas de luz). Esses modelos foram escolhidos para representar estrelas Be recentes da SMC, com taxas de rotação típicas ( $W=0,81$, Rivinius et al. 2006) e que estão na metade da vida na SP. Ao longo da simulações, foram escolhidos 17 instantes diferentes para o cômputo da transferência radiativa, obtendo as respectivas fotometrias sintéticas. Na tabela 2.2, na sexta coluna, apresentamos os valores de $\alpha \tau$ (Eq. 2.7) para os discos dessas estrelas. Por fim, obteve-se uma grade de modelos que é composta por $11 \times 8 \times 15 \times 3=3960$ curvas de luz, para cada uma das bandas fotométricas $B, V, R$ e $I$.

Uma grade de modelos sem discos (fotosférica) também foi computada. Como o custo computacional é muito inferior aos modelos da grade anterior, optou-se por gerar uma grade mais refinada de parâmetros estelares (tabela 2.3), objetivando uma melhor determinação

Tabela 2.3 - Parâmetros da grade de modelos fotosféricos.

\begin{tabular}{ccccc}
\hline$Z$ & $M\left[M_{\odot}\right]$ & $W$ & $t / t_{\mathrm{MS}}$ & $i\left[^{\circ}\right]$ \\
\hline 0,002 & 2,50 & 0,447 & 0,00 & 00,0 \\
& 3,68 & 0,633 & 0,20 & 27,3 \\
& 4,85 & 0,775 & 0,40 & 38,9 \\
& 6,00 & 0,894 & 0,60 & 48,2 \\
& 7,15 & 0,949 & 0,80 & 56,3 \\
8,29 & & 1,00 & 63,6 \\
9,42 & & & 70,5 \\
10,54 & & & 77,2 \\
11,66 & & & 83,6 \\
12,78 & & & 90,0 \\
13,89 & & & \\
15,00 & & & \\
20,00 & & & \\
\hline
\end{tabular}


dos parâmetros estelares. Rímulo et al. (2018) utilizaram 13 valores de massa, 5 taxas de rotação, 6 idades na SP e 10 valores de $\cos i$ (os valores dos dois últimos parâmetros são igualmente espaçados), totalizando $13 \times 5 \times 6 \times 10=3900$ modelos fotosféricos nos quatro filtros.

\subsection{Modelagem de curvas de luz por inferência Bayesiana}

\subsubsection{Pipeline semi-automática para as curvas de luz de estrelas Be}

O parâmetro de tempo adimensional $\tilde{\tau}$ (Eq. 2.8) foi introduzido para que seja possível separar a solução da Eq. 2.1 da variação temporal de $\tau(t)$ (Eq. 2.7). Seguindo os resultados de Ghoreyshi et al. (2018), a metodologia desenvolvida por Rímulo et al. (2018) permite que a viscosidade tenha valores diferentes na fase de construção e destruição do disco. Dessa forma, a equação de transformação entre $\tilde{\tau}$ e $t$ é dada por

$$
\tilde{\tau}=\left\{\begin{array}{lr}
\alpha_{\mathrm{bu}} \frac{t-t_{1}}{\alpha \tau}, & t_{1} \leq t<t_{2} \\
\alpha_{\mathrm{bu}} \frac{t_{2}-t_{1}}{\alpha \tau}+\alpha_{\mathrm{d}} \frac{t-t_{2}}{\alpha \tau}, & t \geq t_{2}
\end{array},\right.
$$

onde consideramos que, para um dado evento de disco, a fase de construção se inicia em $t_{1}$, e termina em $t_{2}$, quando a dissipação se inicia.

Devido aos efeitos de ângulo de visada, as curvas de luz de estrelas Be que exibem eventos de disco podem ser separadas em três grupos:

a) Curvas de luz pole-on: estrelas vistas com ângulos de inclinação pequenos $(0 \leq i \lesssim$ $70^{\circ}$ ), que correspondem, estatisticamente, à maioria das curvas observadas;

b) Curvas de luz edge-on: estrelas shell $\left(i \simeq 90^{\circ}\right)$;

c) Curvas de luz intermediárias: estrelas vistas de ângulos intermediários $(70 \lesssim i \lesssim$ $\left.85^{\circ}\right)^{7}$

Como dito anteriormente, estrelas que pertencem ao grupo $a$, produzem um bump fotométrico; estrelas que pertencem ao grupo $b$ produzem um dip; e no caso do grupo $c$, as variações de brilho aparente são as menores observadas. Nosso interesse está voltado para os dois primeiros grupos.

\footnotetext{
${ }^{7}$ A extensão da região intermediária varia dependendo da banda fotométrica em consideração. Maiores detalhes podem ser encontrados em Rímulo (2017).
} 
De forma geral, uma curva de luz apresentará um excesso em magnitude absoluta, que pode ser positivo ou negativo, em uma banda fotométrica genérica, $X$, que pode ser generalizado pela equação

$$
M_{X}(\tilde{\tau})=M_{X \star}+\Delta X(\tilde{\tau})
$$

onde $M_{X \star}$ é a magnitude absoluta da estrela sem disco, na banda fotométrica $X, \Delta X(\tilde{\tau})$ é a diferença de magnitude causada pelo disco (que pode ser positivo ou negativo).

Substituindo os valores de $\tilde{\tau}$ da Eq. 2.24, nas expressões de solução das equações diferenciais que regem o comportamento fotométrico das curvas de luz, nas fases de dissipação e construção do disco, obtemos

$$
\Delta X(t)=\left\{\begin{array}{lr}
\Delta X_{\mathrm{bu}}^{\infty}\left(1-\frac{1}{\left.1+\left[C_{\mathrm{bu}}\left(t-t_{1}\right)\right]^{\eta_{\mathrm{bu}}}\right),} \quad t_{1} \leq t<t_{2}\right. \\
\Delta X_{\mathrm{bu}}^{\infty}\left(1-\frac{1}{\left.1+\left[C_{\mathrm{bu}}\left(t_{2}-t_{1}\right)\right]^{\eta_{\mathrm{bu}}}\right) \frac{1}{1+\left[C_{\mathrm{d}}\left(t-t_{2}\right)\right]^{\eta_{\mathrm{d}}}},} \quad t \geq t_{2}\right.
\end{array},\right.
$$

onde

$$
C_{\mathrm{bu}}=\alpha_{\mathrm{bu}} \frac{\xi_{\mathrm{bu}}}{\alpha \tau}
$$

$\mathrm{e}$

$$
C_{\mathrm{d}}=\alpha_{\mathrm{d}} \frac{\xi_{\mathrm{d}}}{\alpha \tau}
$$

são coeficientes relacionados às taxas de variação fotométrica, $\xi$ são determinados e tabelados mediante um ajuste com as curvas de luz sintéticas. Os coeficientes $\eta$ são empiricamente determinados de forma a produzirem o melhor ajuste de curvas de luz (para mais detalhes, veja seção 3.3 e 3.4 de Rímulo et al. 2018). O valor $\Delta X_{\text {bu }}^{\infty}$ é o excesso fotométrico do disco quando este está em estado estacionário, ou seja, quando o tempo de construção do disco tende a infinito.

\section{A Pipeline de ajuste}

O nosso objetivo é ajustar uma curva de luz observada com a equação 2.26 para obter, de forma auto-consistente, todos os parâmetros estelares e de disco de interesse. Para isso, a seguinte cadeia de operações é realizada:

I) Encontrar uma curva de luz de uma estrela Be que possua pelo menos uma fase inativa e um bump fotométrico completo;

II) Obter as magnitudes na fase inativa, $X_{\star}$. Ao subtrair essa magnitude da curva de luz, obtemos o excesso, $\Delta X(t)$, que ocorre durante os bumps. No caso de uma curva 
de luz sem o nível fotosférico bem definido não é possível saber a contribuição do bump para o fluxo total. Além disso, é necessário que a fase de construção esteja completamente identificada: a equação utilizada para o ajuste dos dados precisa dos instantes de tempo em que se dá o início da construção $\left(t_{1}\right)$ e dissipação $\left(t_{2}\right)$ do disco;

III) Ajustar a equação 2.26 para os bumps selecionados, obtendo os coeficientes $\Delta X_{\mathrm{bu}}^{\infty}$, $C_{\text {bu }}$ e $C_{\mathrm{d}}$ bem como os tempos $t_{1}$ e $t_{2}$ para as fases de construção e dissipação do disco;

IV) Obter a magnitude absoluta, $M_{X \star}$, da fase inativa, $X_{\star}$, pela correção da distância e do avermelhamento para todas as bandas;

V) Estimar os parâmetros estelares $\left(M, W\right.$ e $\left.t / t_{\mathrm{ms}}\right)$, geométricos $(\cos i)$ e do bump $\left(\Sigma_{0}\right.$, $\alpha_{\text {bu }}$ e $\alpha_{\mathrm{d}}$ para cada bump) que melhor reproduzem os parâmetros estelares $\left(M_{X \star}\right)$ e do bump ajustados $\left(\Delta X_{\mathrm{bu}}^{\infty}, C_{\mathrm{bu}}\right.$ e $\left.C_{\mathrm{d}}\right)$ - vide Eq. 2.27 e 2.28 .

Ressaltamos que no quarto procedimento acima, a magnitude absoluta é necessária para estimar os parâmetros estelares. A partir desses valores, pode-se estimar $\alpha \tau$ (vide Eq. 2.7). Se os parâmetros estelares são conhecidos previamente (e.g., por análise espectroscópica), este procedimento não é mais necessário.

Modelagem utilizando o método de Monte Carlo via cadeias de Markov

O quinto passo descrito acima, que envolve a modelagem dos parâmetros estelares $\left(M_{X \star}\right)$ e de disco $\left(\Delta X_{\mathrm{bu}}^{\infty}, C_{\mathrm{bu}}\right.$ e $\left.C_{\mathrm{d}}\right)$ medidos, foi desempenhado utilizando o método de amostragem de Monte Carlo via cadeias de Markov, $\mathrm{MCMC}^{8}$.

Na metodologia desenvolvida por Rímulo et al. (2018), há $4+5 N_{\text {bumps }}$ parâmetros para cada curva de luz contendo $N_{\text {bumps }}$ bumps identificados. Os quatro primeiros parâmetros, são três estelares $\left(M, t / t_{\mathrm{ms}}\right.$ e $\left.W\right)$ e um geométrico $(\cos i)$, fixos para cada curva de luz. Para cada bump há os seguintes parâmetros: a densidade superficial assintótica de base do disco $\left(\Sigma_{0}\right)$, os tempos iniciais da construção e dissipação do disco (respectivamente, $t_{1}$ e $\left.t_{2}\right)$, e os parâmetros de viscosidade do disco para as fases de construção e dissipação $\left(\alpha_{\mathrm{bu}}\right.$ e $\alpha_{\mathrm{d}}$, respectivamente).

\footnotetext{
${ }^{8}$ Markov Chain Monte Carlo.
} 
O amostrador MCMC utilizado é o emcee, em Python (Foreman-Mackey et al., 2013). O código amostra uma grande coleção de modelos, variando os parâmetros destes dentro de um intervalo previamente estabelecido. Através de uma probabilidade, L(dados $\mid$ modelo), e uma distribuição a priori, $\pi$ (modelo), o amostrador fornece uma distribuição do modelo conforme uma distribuição posterior, $p$ (modelo|dados), dada por

$$
p(\text { modelo } \mid \text { dados }) \propto L(\text { dados } \mid \text { modelo }) \pi(\text { modelo })
$$

A distribuição assumida para os erros nos dados é Gaussiana, então a probabilidade de uma estrela Be com determinados parâmetros de modelo, segundo os dados observacionais, é

$$
L(\text { dados } \mid \text { modelo }) \propto e^{-\frac{\chi^{2}}{2}}
$$

onde, $\chi$ é definido como

$$
\chi^{2}=\chi_{\text {sem disco }}^{2}+\chi_{\text {bump }}^{2}
$$

no qual,

$$
\chi_{\text {sem disco }}^{2}=\sum_{\text {bandas }} \frac{\left(M_{X \star}^{\text {modelo }}-M_{X \star}^{\text {obs }}\right)^{2}}{\sigma^{2}\left(M_{X \star}^{\text {obs }}\right)},
$$

$\mathrm{e}$

$$
\chi_{\text {sem disco }}^{2}=\sum_{\text {bandas bumps }} \sum_{t} \frac{1}{N_{t}} \sum_{i=1}^{N_{t}} \frac{\left(\Delta X_{i}^{\text {modelo }}-\Delta X_{i}^{\text {obs }}\right)^{2}}{\sigma^{2}\left(\Delta X_{i}^{\text {obs }}\right)},
$$

em que $N_{t}$ é o número de pontos de dados para um determinado bump em uma das bandas fotométricas.

A distribuição a priori, $\pi$, representa o nosso conhecimento prévio sobre a distribuição de estrelas Be. É assumido que

$$
\pi(\text { modelo }) \propto M^{-2,3} f_{\mathrm{Be}}(M) e^{-\frac{(W-\langle W\rangle)^{2}}{2 \sigma_{W}^{2}}},
$$

onde o fator $M^{-2,3}$ é a função de massa inicial, IMF, de Kroupa (2001) e $f_{\mathrm{Be}}(M)$ é a fração de estrelas Be, com relação à estrelas B (estimado por Martayan et al. 2007). O fator Gaussiano considera a distribuição das velocidades de rotação de estrelas Be, estimada a partir de Rivinius et al. (2006), onde é encontrado $\langle W\rangle=0,81 \pm 0,12$.

A implementação emcee para o MCMC utiliza múltiplas cadeias de Markov (também chamadas de "walkers"). A posição inicial desses walkers no espaço de parâmetros é aleatoriamente sorteada, considerando intervalos previamente escolhidos pelo usuário. O espaço de parâmetros é percorrido, de forma que, para cada walker, a próxima posição depende da 
atual, levando em consideração a minimização do valor de $\chi^{2}$. Esse procedimento é repetido várias vezes até que um estado estacionário é atingido. Por fim, as posições finais dos walkers se encontram próximas às regiões de maior probabilidade dos parâmetros a serem ajustados. O número de walkers utilizados por Rímulo et al. é proporcional ao número de $4+5 N_{\text {bump }}$ parâmetros dos modelos. Os observáveis estelares e do bump são calculados por uma interpolação linear multidimensional da grade de modelos. A determinação do número de repetições mínimas necessários para a convergência de uma distribuição estacionária, dentro de um erro aceitável é, em geral, um problema de tentativa e erro. Os autores identificaram que aproximadamente $10^{3}$ interações são suficientes para todos os modelos apresentados por Rímulo et al. (2018) cheguem em distribuições estacionárias.

Agora passamos a uma breve revisão do resultado da modelagem de uma das estrelas analisadas por Rímulo et al. (2018). O leitor interessado em maiores detalhes, deve consultar o estudo original.

Na Fig. 2.8 exibimos o resultado da modelagem da estrela SMC_SC1 17701, obtida por Rímulo et al. (2018). Para essa estrela há dados suficientes para as bandas $I$ e $V$, o que permite a modelagem das duas curvas de luz simultaneamente. Os tempos de início da construção $\left(t_{1}\right)$ e dissipação $\left(t_{2}\right)$ do disco são parâmetros ajustados pelo emcee, mas uma estimativa inicial do intervalo que devem variar são fornecidos para o código via inspeção visual da curva de luz. Essas estimativas são mostradas pelas linhas horizontais roxa e laranja no painel superior direito. Na amostragem por MCMC, após um número suficiente de iterações, uma amostra estacionária é obtida, para a qual os parâmetros do modelo estão mais concentrados nas regiões onde a probabilidade posterior é maior. Após atingir o estado estacionário, 100 curvas são aleatoriamente mostradas em conjunto com os dados fotométricos, no painel superior direito. A distribuição das curvas fornece uma estimativa visual da qualidade do ajuste. A qualidade do ajuste pode ser estimada a partir das distribuições posteriores dos parâmetros ajustados mostrados na Fig. 2.8. A diagonal principal (do painel inferior) exibe essa distribuição para cada um dos parâmetros estelares $\left(M, W\right.$ e $\left.t / t_{\mathrm{ms}}\right)$, geométrico $(\cos i)$ e do bump $\left(\Sigma_{0}, \alpha_{\mathrm{bu}}\right.$ e $\left.\alpha_{\mathrm{d}}\right)$, podendo ser utilizados para verificar quão bem determinado cada parâmetros está. As correlações entre os parâmetros são exibidas nas imagens fora da diagonal.

Os parâmetros estelares são basicamente restringidos pela magnitude na fase inativa. As distribuições alargadas dos três histogramas da esquerda mostram que esses valores 


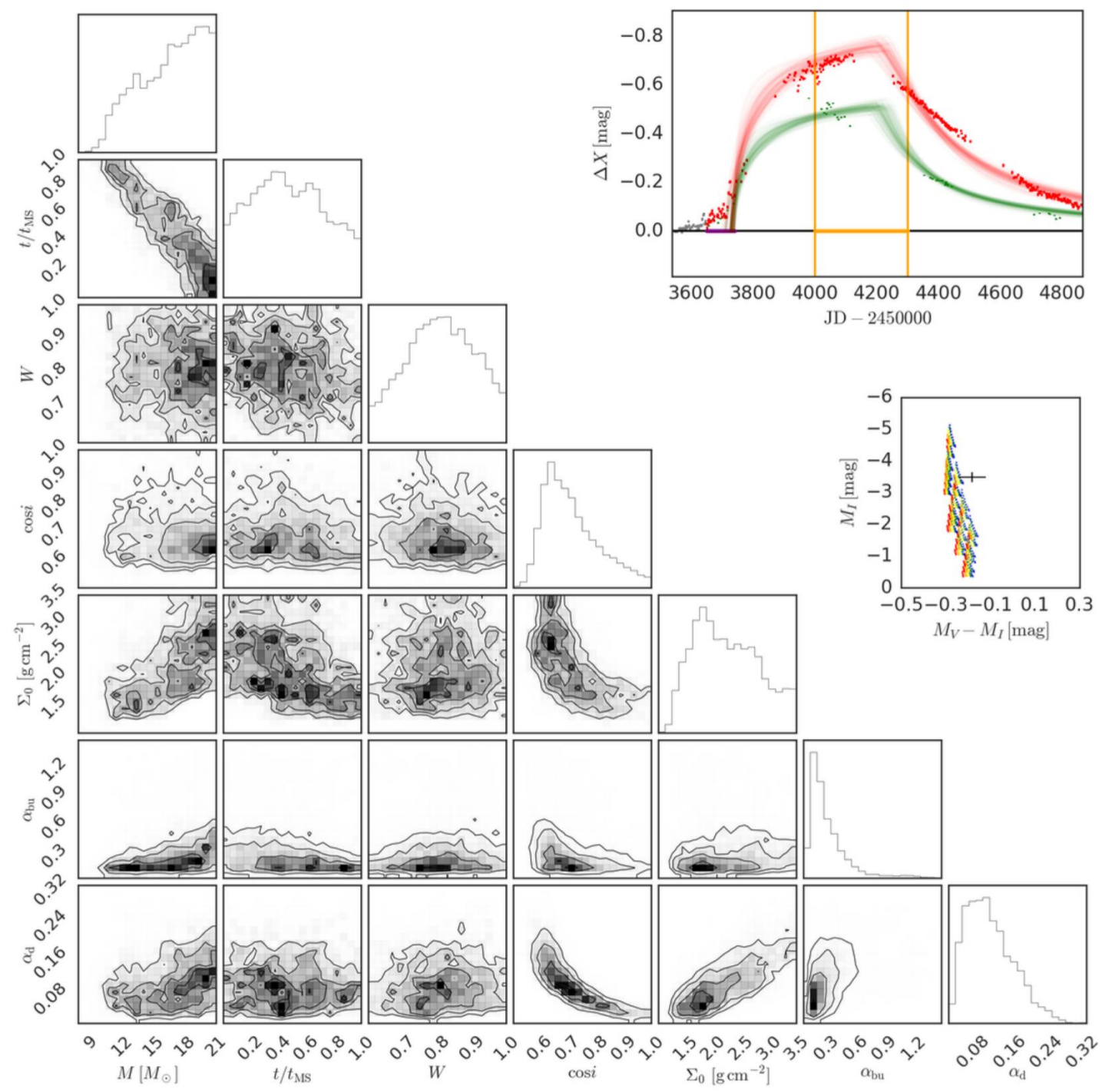

Figura 2.8: Direita superior: Curva de luz da estrela SMC_SC1 17701. Linhas finas: os 100 curvas de modelos aleatoriamente selecionadas a partir da amostra estacionária do código emcee. A cor vermelha (verde) indicam a banda fotométrica $I(V)$. Os intervalos de tempo roxo e laranja marcados em linhas horizontais são os intervalos permitidos para os parâmetros $t_{1}$ e $t_{2}$ do modelo, respectivamente. Direita intermediário: Diagrama cor-magnitude mostrando a grade de modelos de estrelas Be inativas e a posição de SMC_SC1 17701. Inferior: Resultados para a simulação do emcee para SMC_SC1 17701. O histograma das distribuições das probabilidades posteriores são mostrados nos painéis superiores e as correlações dois-a-dois dos parâmetros estelares $\left(M, W\right.$ e $\left.t / t_{m s}\right)$, geométrico $(\cos i)$ e do bump $\left(\Sigma_{0}, \alpha_{\mathrm{bu}}\right.$ e $\left.\alpha_{\mathrm{d}}\right)$ são mostrados nos painéis fora da diagonal. Os parâmetros $t_{1}$ e $t_{2}$ não são mostrados por conveniência. Os níveis de densidade normalizada mostrados nos painéis fora da diagonal são $12 \%, 39 \%, 68 \%, 87 \%$ do pico da probabilidade.

não estão bem determinados, cujas estimativas obtidas são de $M=17,0_{-3,9}^{+2,8} M_{\odot}, t / t_{\mathrm{ms}}=$ $0,5_{-0,3}^{+0,3}$ e $W=0,81_{-0,11}^{+0,11}$. Aproximadamente, a amplitude do bump depende de $\Sigma_{0}$, além de $\cos i$, enquanto que a viscosidade basicamente controla quão rápido ocorre a variação fotométrica. Os valores ajustados são de $\Sigma_{0}=2,2_{-0,5}^{+0,7} \mathrm{~g} \mathrm{~cm}^{-2}, \alpha_{\mathrm{bu}}=0,25_{-0,09}^{+0,21}, \alpha_{\mathrm{d}}=$ 
$0,11_{-0,04}^{+0,06}$. Os valores da taxa de perda de massa e momento angular são de $\left(-\frac{\partial M}{\partial t}\right)_{\text {typ }}=$ $1,11_{-0,51}^{+1,24} \times 10^{-9} \mathrm{M}_{\odot}$ ano $^{-1}$ e $\left(-\frac{\partial J}{\partial t}\right)_{\text {std }}=2,33_{-1,17}^{+2,92} \times 10^{36} \mathrm{~g} \mathrm{~cm}^{2} \mathrm{~s}^{-2}$.

Em suma, a Fig. 2.8 ilustra a capacidade que a pipeline descrita acima tem de extrair os parâmetros físicos e geométricos relevantes de uma estrela e seu disco, a partir da curva de luz. Este é um dos principais resultados do trabalho de doutorado de Leandro R. Rímulo, pois permite o exame de um grande número de curvas de luz visando estudos de amostras estatisticamente significativas. Os resultados do emprego desta pipeline aos dados selecionados para a LMC (Cap. 3) são descritos no capítulo 4. 
Capítulo 3

\section{Curvas de luz de estrelas Be da $L M C$}

\subsection{Levantamentos fotométricos}

Uma das melhores formas de estimar o parâmetro de viscosidade é estudar o comportamento do disco onde o efeito difusivo da viscosidade terá uma contrapartida observacional na variabilidade temporal da SED. Portanto, curvas de luz de cobertura temporal extensa, tais como as oriundas de levantamentos de microlentes gravitacionais e de trânsitos planetários, são excelentes objetos de estudo para a análise dos processos dinâmicos que ocorrem na formação e dissipação de discos de estrelas Be. Como os estudos de Rímulo et al. (2018) apontam, outra vantagem de estudar curvas de luz é a possibilidade de estimar as taxas de perda de massa e momento angular, parâmetros que são importantes para modelos evolucionários de estrelas em alta rotação.

Em 1986, o astrônomo polonês Bohdan Paczyński propôs o uso de microlentes gravitacionais para o estudo de matéria escura na forma de objetos compactos massivos do halo $\left(\mathrm{MACHOs}^{1}\right.$ ). Paczyński foi iniciador do projeto OGLE ${ }^{2}$ (experimento de lentes gravitacionais no óptico, liderado por Andrzej Udalski) e do ASAS ${ }^{3}$ (levantamento automatizado de todo o céu, criado em parceria com Grzegorz Pojmański). Levantamentos de microlentes gravitacionais como o OGLE (Udalski et al., 1992), MACHO (Alcock et al., 1997) e EROS (Aubourg et al., 1993) têm como subproduto curvas de luz de milhões de estrelas.

Este capítulo é dedicado à descrição de características das curvas de luz de candidatas a estrelas Be da LMC. Milhares de curvas de luz foram obtidas nos levantamentos OGLE-II (Udalski et al., 1997), OGLE-III (Udalski 2003; Udalski et al. 2008) e OGLE-IV (Udalski

\footnotetext{
${ }^{1}$ Massive Compact Halo Objects.

${ }^{2}$ Optical Gravitational Lensing Experiment.

${ }^{3}$ All Sky Automated Survey.
} 

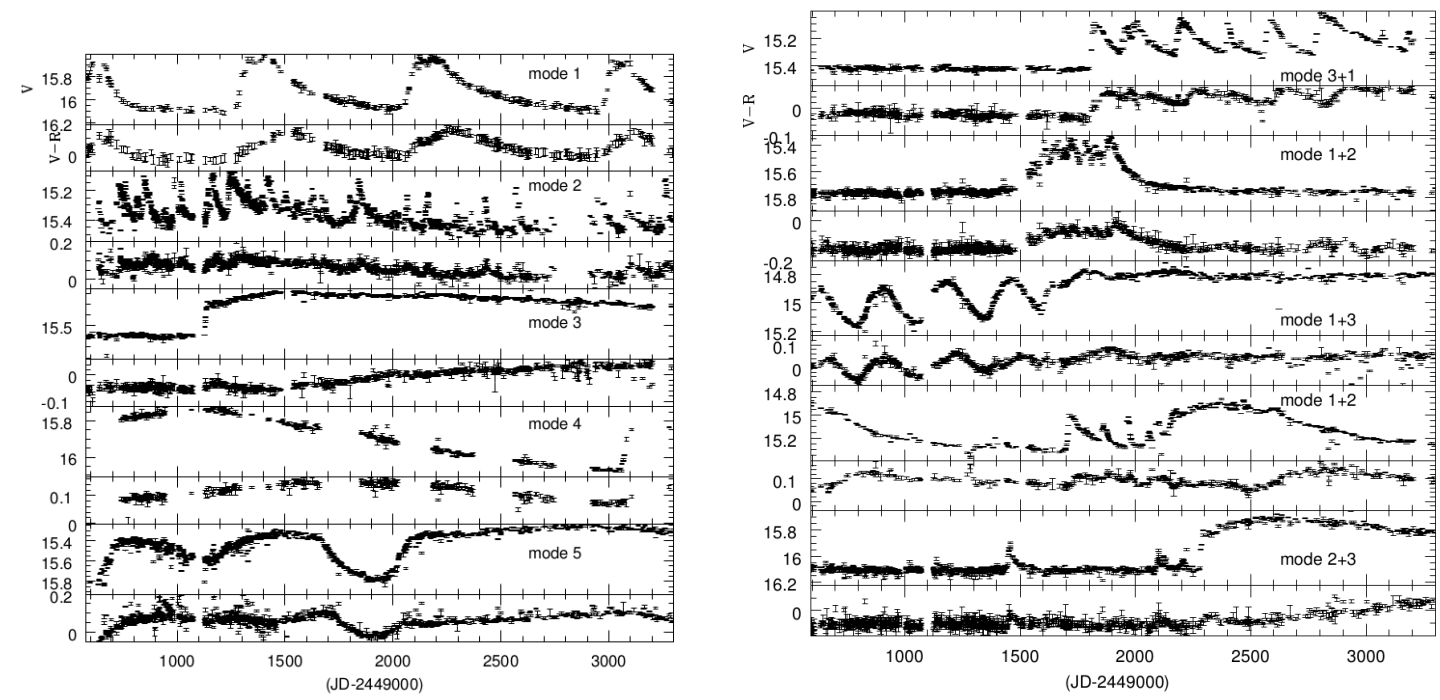

Figura 3.1: Esquerda: Curvas de luz na banda $V$ e de cores $V-R$ que exemplificam as cinco modos de variabilidade exibidas na amostra de estrelas azuis variáveis. Direita: Exemplos de dados que exibem mais de um dos modos de variabilidade identificados. Fonte: Keller et al. (2002).

et al., 2015), para candidatas a estrelas Be da LMC (Sabogal et al., 2005). A partir dessa amostra, selecionamos 83 curvas de luz que continham fases claras de inatividade, além de ao menos um bump ou dip bem definido (ver seção 2.1.2), totalizando 120 bumps e dips. Nas seções seguintes damos mais detalhes sobre os levantamentos utilizados, os critérios de seleção da nossa amostra, bem como uma descrição estatística das propriedades das curvas de luz selecionadas.

\subsection{Levantamentos existentes de curvas de luz de estrelas Be das Nuvens de Magalhães}

Keller et al. (2002) selecionaram 1279 curvas de luz de estrelas Be variáveis da LMC para o levantamento fotométrico MACHO. Para a amostra final, foram selecionadas estrelas cujas as posições no diagrama de cor-magnitude correspondessem às posições de estrelas B. Além disso, foram selecionados automaticamente dados de estrelas que satisfizessem certos critérios de variabilidade. Contaminantes da amostra (e.g., binárias eclipsantes) foram excluídos por inspeção visual. Na amostra, os autores identificaram cinco modos diferentes de variabilidade, ilustrados na Fig. 3.1:

1. Eventos de Bump: tipicamente, eventos com variação de magnitude $\Delta V \sim 0,2-$ 0,4 mag em amplitude, e duração de 100 a 800 dias; 


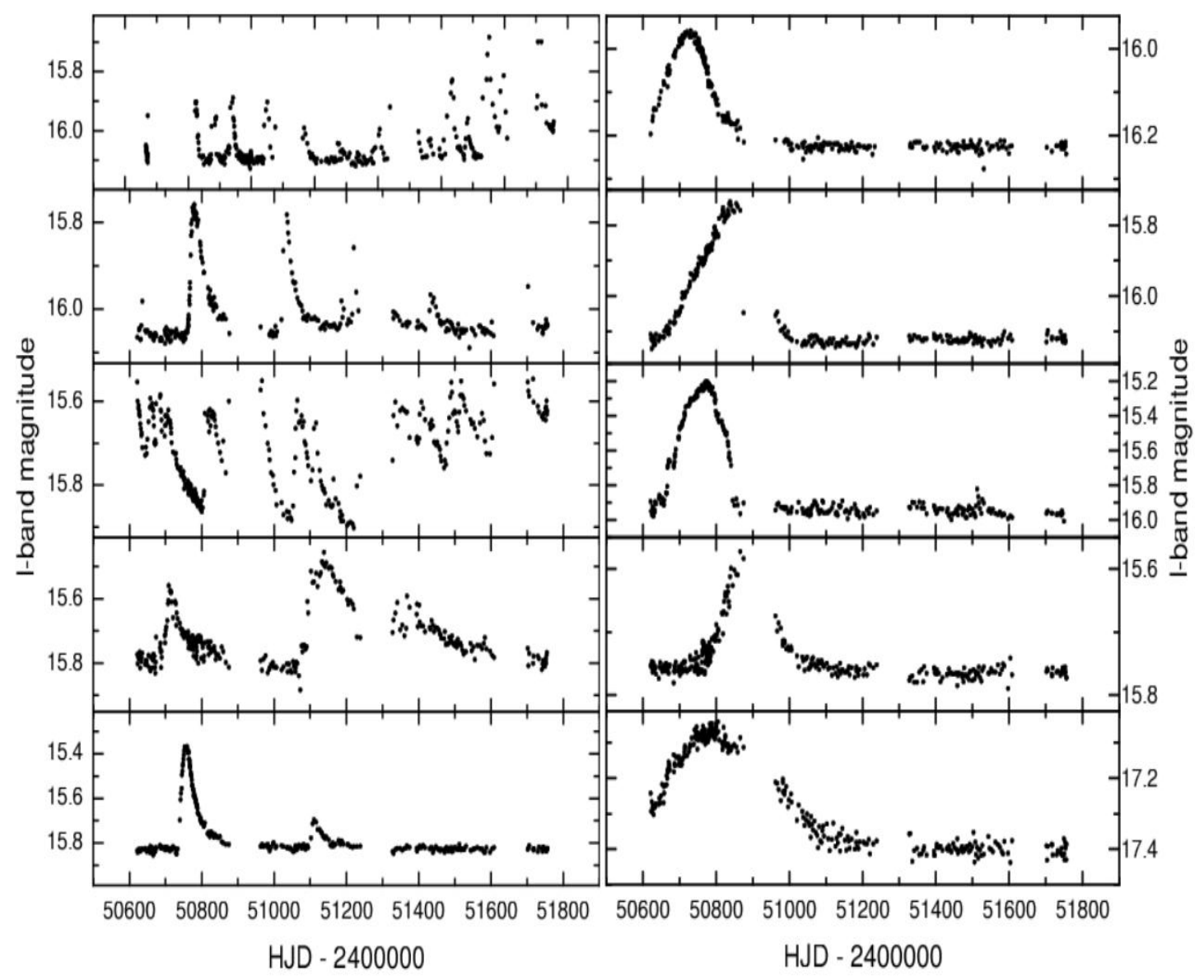

Figura 3.2: Exemplos de curvas de luz na banda $I$ para estrelas candidatas a Be observadas pelo levantamento OGLE-II. Esquerda: estrelas tipo 1 exibindo eventos "agudos". Direita: estrelas que apresentam eventos de tipo 1 em formato de corcova. Fonte: Mennickent et al. (2002).

2. Eventos de flicker: São rápidos, com duração entre 10 e 50 dias e de baixa amplitude $(\Delta V \sim 0,05-0,15 \mathrm{mag}) ;$

3. Eventos step: variações de brilho da ordem de $\Delta V \sim 0,2-0,3 \mathrm{mag}$, dentro de um intervalo de $10-50$ dias;

4. Eventos de variações de linha de base: variações que possuem longa duração (centenas de dias), com $\Delta V \sim 0,4$ mag;

5. Eventos de fading: Variações de luminosidade da ordem de $\Delta V \sim 0,4$ mag com durações de $200-600$ dias.

Diferentes modos podem estar presentes na curva de luz de uma mesma estrela, como ilustrado no quadro à direita da figura 3.1. Uma subamostra de 102 estrelas da amostra inicial foi selecionada para observações espectroscópicas em diferentes épocas. A maioria 
desses objetos (91\%) exibia, pelo menos em uma época, linhas de Balmer em emissão e confirmando, desse modo, que pelo menos 90\% dessa subamostra é compostas de estrelas Be.

Utilizando curvas de luz do OGLE-II para a SMC Mennickent et al. (2002) selecionaram 1056 curvas de luz de estrelas candidatas a Be. O método utilizado para seleção é semelhante ao empregado por Keller et al. (2002). Uma classificação morfológica alternativa também é apresentada (veja Figs. 3.2 e 3.3):

- Tipo 1: eventos "agudos" ou em formato de "corcova", correspondendo aos modos 1 e 2 de Keller et al. (2002);

- Tipo 2: estados "altos" e "baixos", similares aos eventos de modo 3 de Keller et al. (2002);

- Tipo 3: eventos quase-periódicos e periódicos;

- Tipo 4: eventos não periódicos.

As frações relativas encontradas para essas curvas são de 64,9\% (tipo 4), 13,2\% (tipo 1), $14,6 \%$ (tipo 2 ) e $7,4 \%$ (tipo 3 ).

Sabogal et al. (2005) deram continuidade ao trabalho ao selecionar candidatas a estrelas Be da LMC. Foram selecionados 2446 objetos a partir da análise de curvas de luz do levantamento OGLE-II. As proporções encontradas para as curvas são de: 60,0\% (tipo 4), $23,1 \%$ (tipo 1), 6, 1\%(tipo 3) e 4,0\%(tipo 2).

Paul et al. (2012) estudaram as propriedades espectrais das estrelas selecionadas por Mennickent et al. (2002) e Sabogal et al. (2005). Para a SMC, a maioria das estrelas de tipo 1 e 2 investigadas eram de tipo espectral B com linhas de emissão características de material circunstelar. Contudo, essas características não foram claramente observadas para as estrelas de tipo 1 da LMC.

\subsection{Curvas de luz de candidatas a estrelas Be}

No trabalho de Rímulo et al. (2018), os autores modelaram os dados fotométricos para alvos da SMC, utilizando os levantamentos OGLE-II e III, empregando a pré-seleção de Mennickent et al. (2002). 

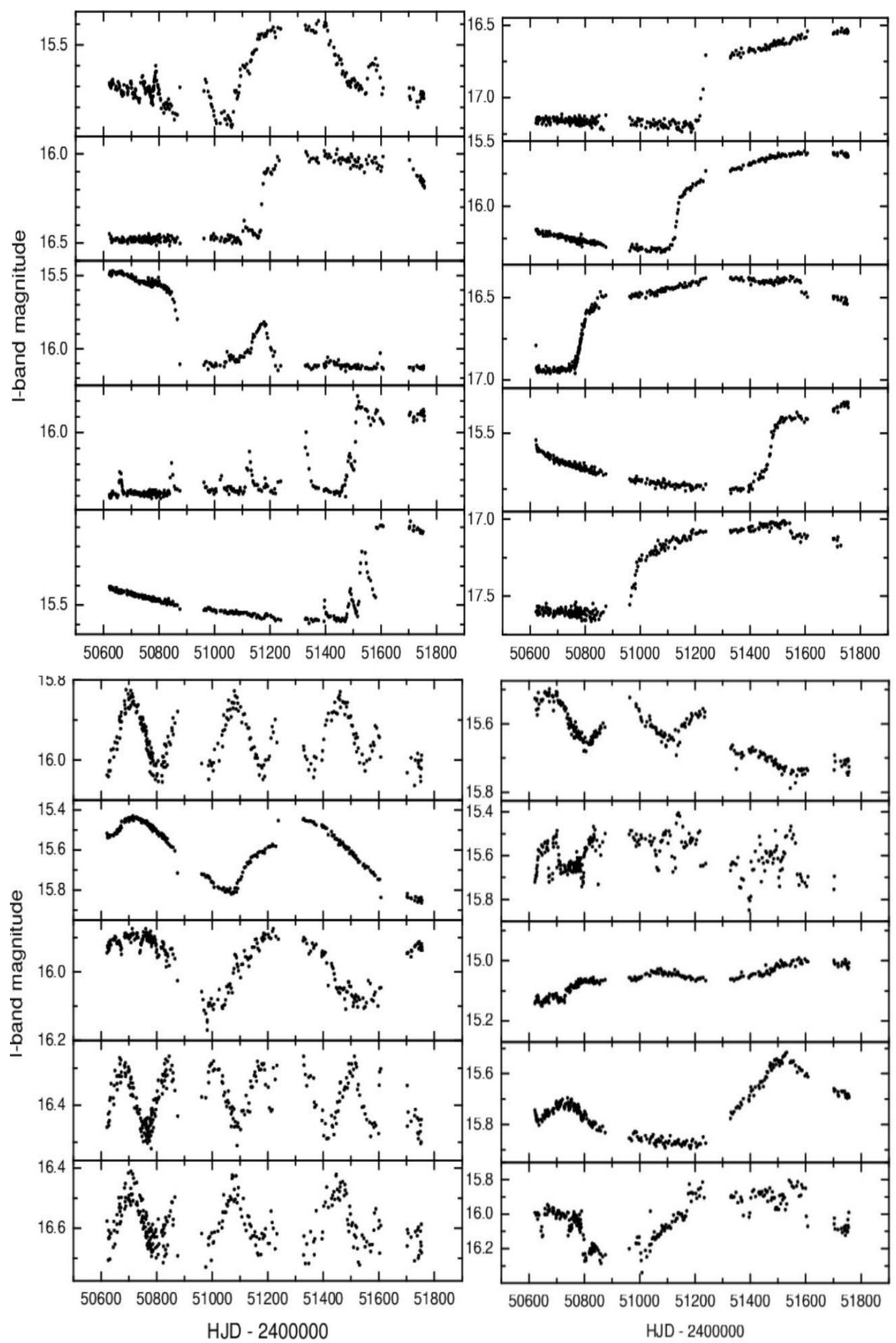

Figura 3.3: Idem à Fig. 3.2 para curvas de luz que possuem características morfológicas de tipos 1 e 2, simultaneamente (painel superior esquerdo), apenas tipo 2 (superior direito), tipo 3 (esquerda inferior) e tipo 4 (direita inferior). Fonte: Mennickent et al. (2002). 
Neste trabalho, nós utilizamos a pré-seleção de Sabogal et al. (2005), feitos apenas com base dos dados do OGLE-II. Já tínhamos disponíveis as curvas de luz da LMC para os levantamentos OGLE-II e III, obtidos juntamente com os dados da SMC supra citados. Devido à problemas de imprecisões fotométricas, a identificação das estrelas entre a fase II do OGLE as demais não pode ser feita de forma automatizada. Uma rotina de identificação cruzada foi desenvolvida para reconhecer os alvos de interesse nas diferentes fases observacionais. A partir da identificação dos alvos, obtivemos os dados do levantamento IV do OGLE para as bandas $I, B$ e $V$ (uma fração pequena das estrelas possuem dados nessas duas últimas bandas), com o auxílio de nosso colaborador, Igor Soszyński. Os dados do OGLE-II cobrem o período de janeiro de 1997 a maio de 2000. O dados correspondentes ao OGLE-III obtidos entre junho 2001 e maio de 2009. A fase IV do projeto aconteceu entre março de 2010 e junho de 2017.

\section{Rotina de identificação cruzada}

As estrelas observadas nos levantamentos OGLE-III e IV podem ser identificadas diretamente, porém o mesmo não ocorre para a fase II. Isso se deve às alterações instrumentais entre as fases II e III. Para encontrar as correspondências entre as estrelas pré-selecionadas por Sabogal et al. (2005), fizemos uma identificação cruzada entre a lista de Sabogal et al. $(\text { fase II })^{4}$ com os alvos da fase III, através de uma rotina Python desenvolvida para este fim.

Para fazer a seleção de potencial correspondência entre as duas listas, utilizamos a distância angular dada por

$$
\theta=\cos ^{-1}\left[\operatorname{sen}\left(\delta_{1}\right) \operatorname{sen}\left(\delta_{2}\right)+\cos \left(\delta_{1}\right) \cos \left(\delta_{2}\right) \cos \left(\alpha_{1}-\alpha_{2}\right)\right]
$$

onde $\alpha$ e $\delta$, respectivamente, correspondem à ascensão reta e declinação das diferentes estrelas, denotadas pelos diferentes subíndices.

Para que um objeto fosse considerado potencialmente correspondente a uma das estrelas de interesse, a sua distância angular deveria ser $\theta \leq 3$ ". Para a maioria das estrelas de interesse, foram encontradas correspondência múltipla. Estrelas que possuíam poucas observações e cujas diferenças entre as magnitudes fotométricas médias medidas fossem superiores a $1 \mathrm{mag}$ do valor observado durante a fase II foram eliminadas. Após eliminar

\footnotetext{
${ }^{4}$ A lista está disponível online em ftp://ftp. astrouw.edu.pl/ogle/ogle3/maps/lmc/maps/.
} 
estrelas incompatíveis, das 2446 estrelas selecionadas por Sabogal et al. (2005), obtivemos 2032 identificações únicas entre as fases observacionais II e III. Restam 767 identificações múltiplas (contando as repetições) que precisam de estudos mais detalhados. Para as 2032 estrelas identificadas, geramos as curvas de luz completas, que foram inspecionadas visualmente visando selecionar aquelas que possuíam bumps ou dips claros, bem como uma fase sem disco bem definida (mais detalhes abaixo). Na seção 3.4, apresentamos a seleção de bumps que foram selecionados. Um estudo da amostra completa de 2032 curvas de luz, como um todo, é apresentada na seção 3.5. Nesta última seção também reportamos a identificação de uma nova fenomenologia, que se convencionou chamar de bumps muito longos, VLB ${ }^{5}$. Adicionalmente, apresentamos uma discussão breve sobre a classificação das curvas de luz.

\subsection{Seleção de bumps para a Grande Nuvem de Magalhães}

Ao se agregar os dados dos diferentes levantamentos OGLE, nota-se que para algumas estrelas, o nível de referência para calibração da fotometria varia. A principal diferença ocorre entre as fases II e as seguintes. Isso se deve às diferentes configurações instrumentais, o que ocasiona diferentes níveis de referência. Por indicação de Igor Soszyński, adotamos como referência os dados de OGLE-III, dado que as magnitudes observadas pelo OGLEIII e OGLE-IV são mais consistentes entre si. Para se efetuar a mudança do ponto zero, realizada para as curvas de luz selecionadas para este estudo, foi necessário identificar as fases de inatividade nos dados correspondentes aos levantamentos OGLE-II, III e IV.

As curvas de luz foram selecionadas por inspeção visual, de acordo com as características apresentadas na seção 2.1.2. A prioridade foi dada para estrelas que possuem eventos de disco bem comportados e com uma fase clara de inatividade. Também se evitou bumps que contivessem a presença de eventos de curta duração, os flickers. A amostra final é composta por 105 estrelas, que abrangem 162 bumps. Dessa amostra, excluímos curvas de luz com problemas de medidas (poucos pontos ou cuja cor $V-I$ era incompatível com o valor esperado). Por fim, modelamos 83 curvas de luz e 120 bumps, que são mostradas na tabela 3.2. Na tabela, as linhas horizontais separam os dados de cada estrela. Cada linha da tabela contém os dados de um bump. A quinta e a sexta colunas mostram o

\footnotetext{
${ }^{5}$ Very Long Bumps
} 


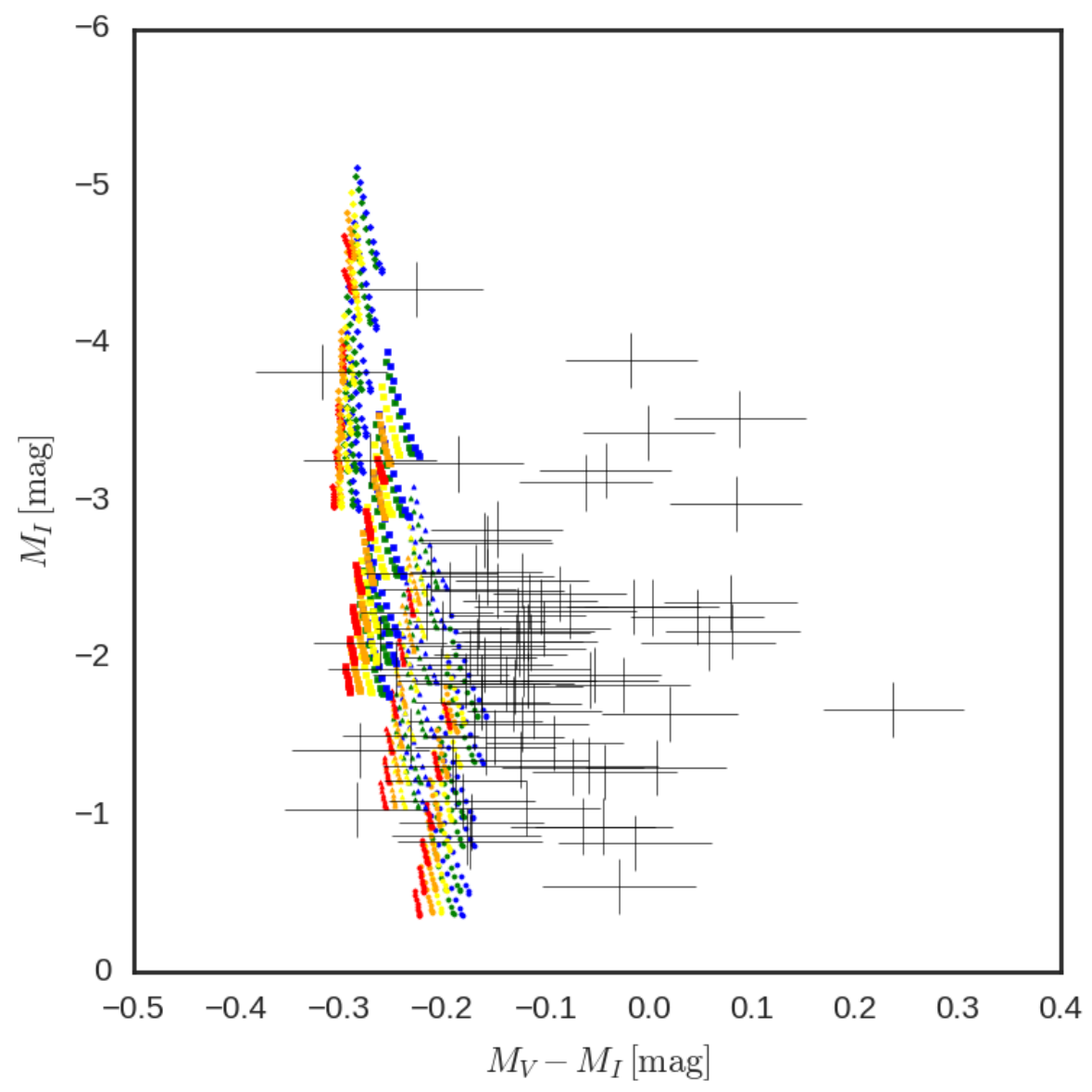

Figura 3.4: Diagrama cor-magnitude de estrelas selecionadas para este estudo (em preto). Note que os valores de I e V mostrados correspondem à fase sem disco. Adicionalmente, mostramos as estrelas sintéticas da grade de modelos fotosférica (veja tabela 2.3). Os modelos de 6,0, 8,29, 11,66 e 15,00 $M_{\odot}$ são representados pelos círculos, triângulos, quadrados e diamantes, respectivamente. Os cinco valores de $W$ correspondem, em ordem crescente, às cores vermelha, laranja, amarela, verde azul. Os cinco valores de idade são identificados como os grupos de pontos que se movem em direção à parte superior direita da figura. Individualmente, o efeito de ir de um ângulo de $90^{\circ}$ para $0^{\circ}$ é a migração em direção ao canto superior esquerdo.

início e o fim da fase inativa selecionada da curva de luz. As magnitudes $B_{\star}, V_{\star}$ e $I_{\star}$ da fase inativa estão contidas nas sexta, sétima e oitava colunas (pela forma como o OGLE foi desenhado, as bandas $B_{\star}$ e $V_{\star}$ não estão disponíveis para todas as estrelas). A décima primeira coluna contém as bandas que foram consideradas para a modelagem dos bumps individualmente. As últimas duas colunas trazem estimativas do intervalo de tempo para a duração do bump.

O nível da emissão fotosférica é utilizado para estimar os parâmetros estelares. Para tanto, é necessário corrigir o avermelhamento e converter as magnitudes aparentes em 
absolutas, dada pela equação

$$
M_{X \star}=X_{\star}-(5 \log d-5)-A_{X} .
$$

A distância utilizada foi de $d=49,97 \pm 1,3 \mathrm{kpc}$ de Pietrzyński et al. (2013).O valor médio de $E(V-I)$ das estrelas RR Lyrae de toda a LMC é de $E(V-I)=0,11 \pm 0,06$ mag (Haschke et al., 2011). O avermelhamento nas bandas $B, V$, e $I$ foram obtidos pelas relações de $A_{X} / A_{V}$ dadas por Gordon et al. (2003).

Na fig. 3.4 apresentamos o diagrama cor-magnitude para a subamostra de estrelas e a grade de modelos sem discos (veja tabela 2.3). Nota-se que a maioria das estrelas está levemente deslocada para a direita se compara com os modelos. Em outras palavras, essas estrelas já teriam saído da SP. Como pode-se notar da Fig. 2.6, na fase pós-SP a estrela tem um aumento de raio considerável o que reduz muito a sua rotação. Por consequência, é esperado que o fenômeno Be cesse logo após a SP. Portanto, a posição das estrelas no diagrama cor-magnitude reflete um valor incorreto assumido para o avermelhamento, o que é plausível pois espera-se que cada linha de visada esteja associada a avermelhamentos diferentes devido às inomogeneidades do meio interestelar.

\subsubsection{Propriedades globais}

Nesta seção apresentamos algumas propriedades globais da amostra de bumps e dips, que abarca 105 estrelas. Destas, 85 (120 bumps) possuem ângulos de inclinação $i \lesssim 70^{\circ}$ e 20 correspondem a $85^{\circ} \lesssim i \simeq 90^{\circ}$ (43 dips). Por conveniência, iremos nos referir aos dois grupos de estrelas como vistas de cima e de lado ou aos bumps e dips, respectivamente. Como as observações para a banda $I$ são mais completas, os valores e gráficos apresentados nessa seção são relativos a esta. Como o critério de seleção atual para os valores dos parâmetros de entrada da pipeline é visual, os valores apresentados são aproximados e correspondem aos valores médios (no caso dos tempos de construção, dissipação e duração dos bumps) ou extremos (para os valores de amplitude, que é estimada a partir da amplitude máxima observada com relação ao valor médio do nível fotosférico). Por essa razão, não apresentamos as barras de erros dessas estimativas. O desenvolvimento de um método analítico para obter esses valores de forma automatizada e mais consistente está entre nossos objetivos futuros.

Na Fig. 3.5 apresentamos histogramas com algumas das propriedades medidas dos 

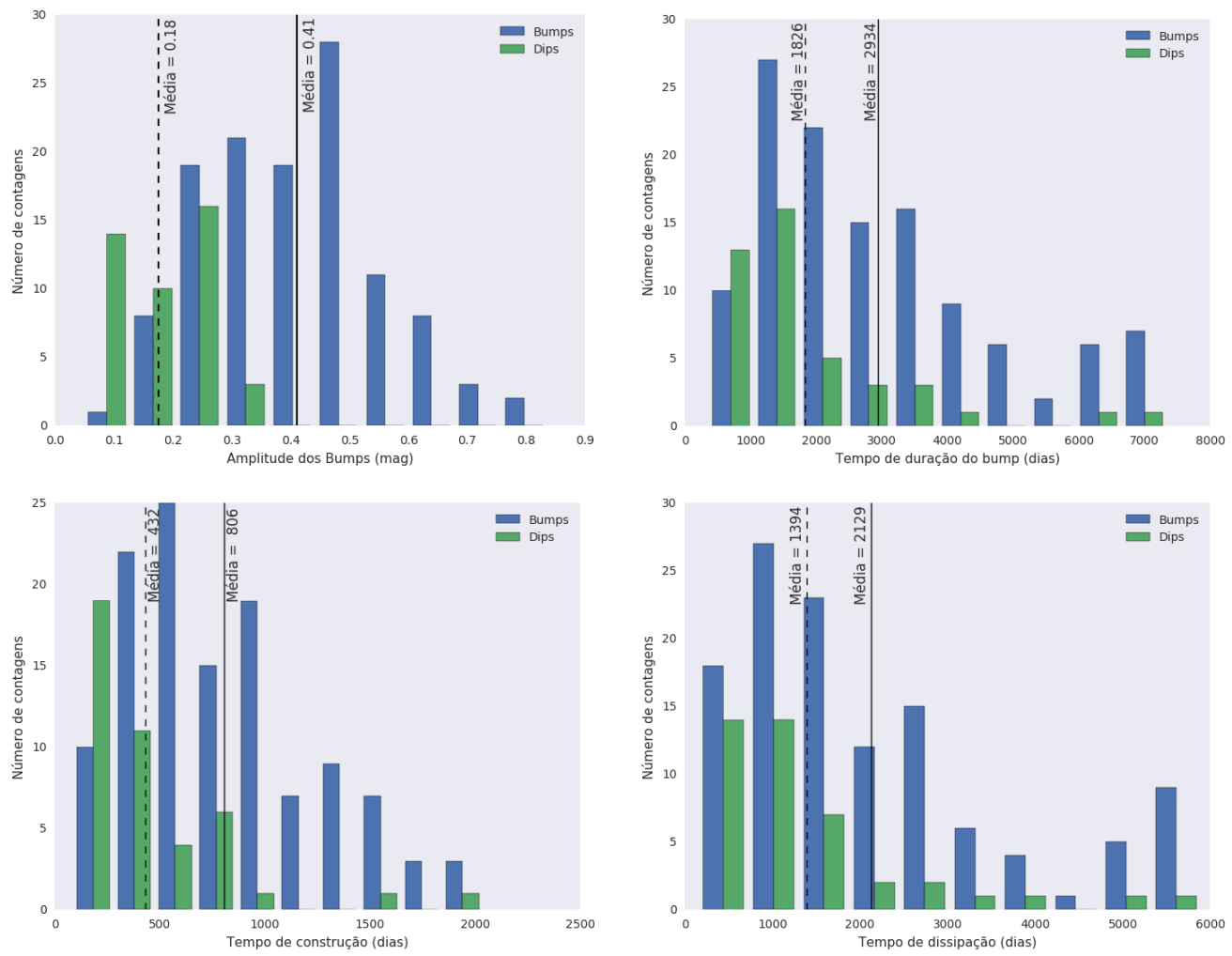

Figura 3.5: Histogramas para os bumps (dips) em azul (verde) e a respectiva média (com seu valor numérico) é apresentada pelas linha contínua (pontilhada). Na linha superior apresentamos as figuras relativas às amplitudes e ao tempo total de duração dos eventos de disco. Os tempos de construção e dissipação dos discos são apresentados de forma separada na linha inferior, nessa ordem.

bumps e dips. No painel superior esquerdo, temos o histograma para a distribuição de amplitudes. As estrelas vistas de cima possuem amplitudes tipicamente maiores do que as vistas de lado. Isso se reflete tanto nos intervalos de amplitude encontrados (de 0,048 a 0,32 e de 0,069 a 0,846 mags) como nos valores médios (0,18 e 0,41 mag) para estrelas vistas de lado e de cima, respectivamente. Como será visto no Cap. 4, esses valores estão em bom acordo com os encontrados em curvas de luz sintéticas de estrelas Be da LMC.

A duração dos bumps também é maior do que a dos dips, como pode ser visto na linha superior direita da Fig. 3.5, cujos valores correspondem a 2934 e 1826 dias, respectivamente (a razão entre as médias correspondem a um fator de 1,6). Labadie-Bartz et al. (2018) realizaram estudo semelhante utilizando observações do KELT ${ }^{6}$ para estrelas Be da Galáxia. O valor encontrado para a duração média dos eventos de disco foi de 24,3 dias. Um ponto de interesse na Fig. 3.5 é o número grande (26) de bumps com duração superior a 5000 dias. Até o momento não encontramos na literatura relatos de bumps tão longos.

\footnotetext{
${ }^{6}$ Kilodegree Extremely Little Telescope.
} 


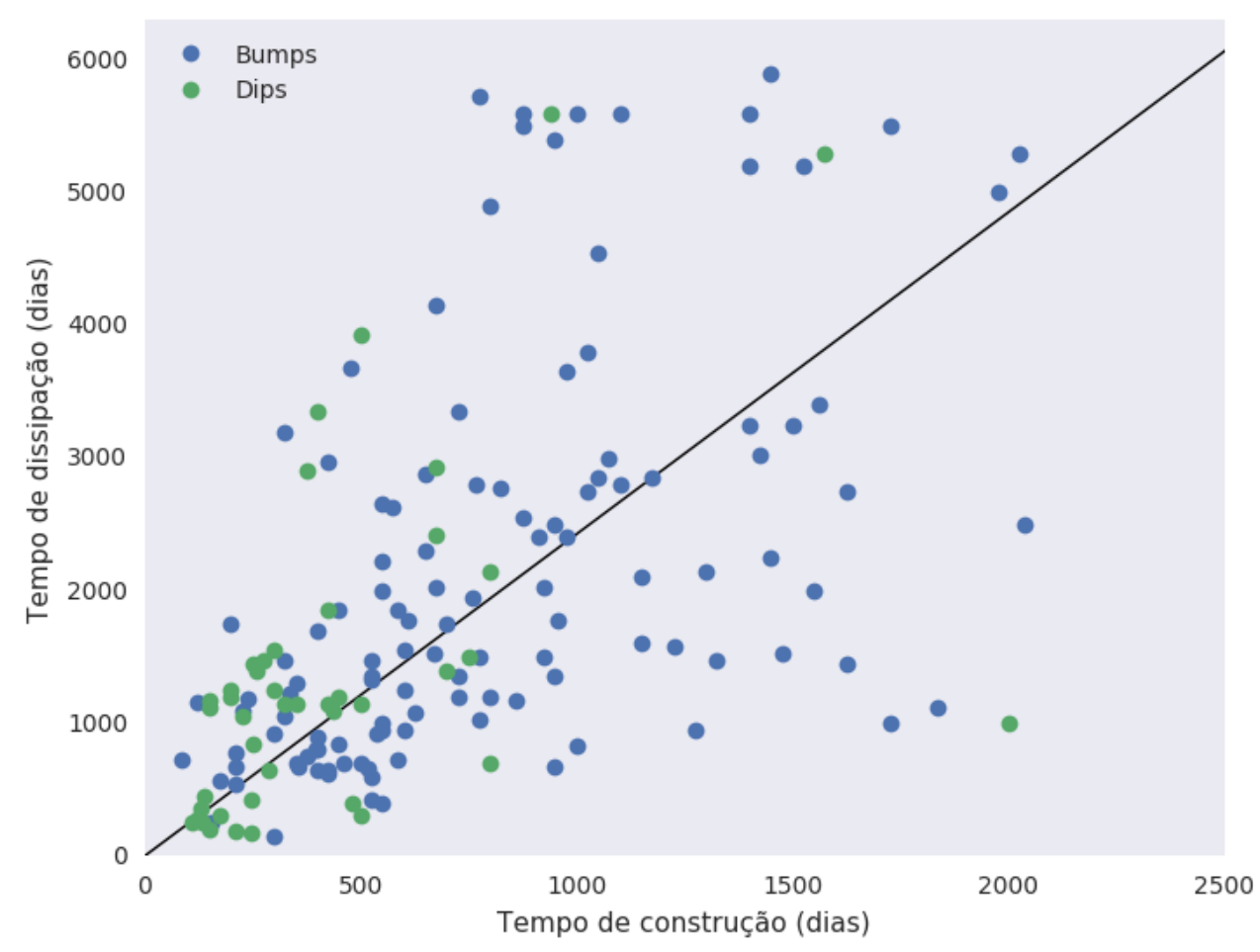

Figura 3.6: Gráfico para o tempo de construção (eixo horizontal) contra a duração da dissipação (eixo vertical). A linha mostra o melhor ajuste aos dados, cujo coeficiente angular encontrado é de 2,43 para toda a amostra.

Na seção 3.4.2 exploramos um pouco mais esses eventos e como o seu estudo pode nos proporcionar uma melhor compreensão das classificações morfológicas das curvas de luz (vide seção 3.6).

$\mathrm{Na}$ linha inferior apresentamos a duração dos eventos de construção e dissipação do disco. Para estimar o valor do tempo de surgimento do disco, medimos o tempo transcorrido desde a alteração do nível da magnitude fotosférica até o momento no qual a fotometria começa a retornar ao valor fotosférico. Esse intervalo corresponde, aproximadamente, ao intervalo no qual a estrela está perdendo massa. Observa-se que o tempo de dissipação é mais longo do que o surgimento do disco, característica semelhante à observada na amostra de Rímulo et al. (2018) para a as curvas de luz da SMC.

Para uma melhor visualização dos respectivos tempos de construção e dissipação, apre-

Tabela 3.1 - Número de estrelas (linha superior) que apresentam o número de bumps apresentado na parte inferior da tabela.

\begin{tabular}{c||ccccc} 
Número de estrelas & 27 & 7 & 2 & 1 & 1 \\
\hline Número de bumps & 2 & 3 & 4 & 5 & 8
\end{tabular}


LMC 111.6.66352

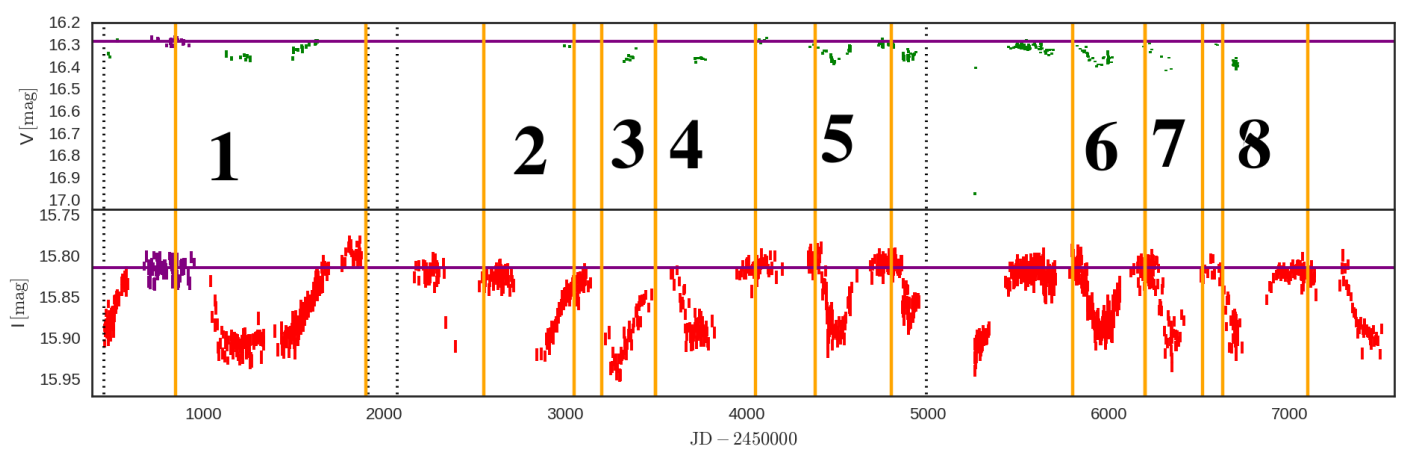

Figura 3.7: O exemplar mais excepcional de curvas de luz do OGLE-II, III e IV com múltiplos eventos de disco. O identificador da estrela é apresentado na parte superior da figura. A banda fotométrica $I(V)$ é mostrada em vermelho (verde). As linhas verticais pontilhadas separam as diferentes fases do levantamento OGLE. As observações mostradas em roxo são assumidas como sendo o nível fotométrico para a fase inativa da estrela (sem disco). O seu valor médio é representado pela linha horizontal roxa. Os pares de linhas verticais laranjas delimitam os bumps selecionados visualmente. Adicionamos números para auxiliar na visualização dos eventos de disco identificados.

sentamos na Fig. 3.6, esses valores para todos os eventos de disco. A reta que melhor representa os dados possui um coeficiente angular correspondente a 2, 43, ou seja, o tempo de dissipação dos discos é, em geral, duas vezes mais longo que o intervalo de construção. Esta relação também foi encontrada por Labadie-Bartz et al. (2018), ao estudar eventos de disco de curvas de luz de estrelas Be da Galáxia, cujos coeficientes obtidos foram de 1,97 (estrelas recentes), 1,88 (médias) e 6,54 (tardias).

\section{Estrelas com múltiplos bumps}

Dentre as 105 curvas de luz selecionadas, várias apresentam mais de um evento de disco isolado (38, o que corresponde a 36\% das estrelas). Na tabela 3.1 apresentamos as estrelas com múltiplos bumps, divididas pelos número de eventos que apresentam. Na Fig. 3.7 apresentamos o caso mais proeminente, a curva de luz da estrela LMC 111.6.66352 ${ }^{7}$, que contém 8 dips.

\subsubsection{Bumps extremamente longos}

Na seção 3.4.1, vimos que um grupo de bumps possuem durações bastantes altas. A Fig. 3.8 apresenta dois exemplos de curvas de luz que contém esse tipo de fenômeno.

\footnotetext{
${ }^{7}$ Quando nos referirmos às estrelas utilizaremos o identificador da fase III do OGLE.
} 
LMC 162.1.30252

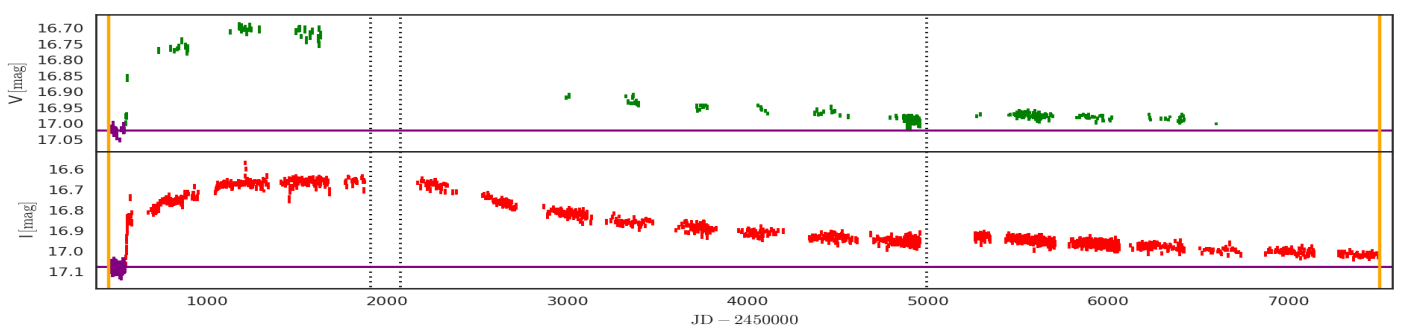

LMC 162.2.38930

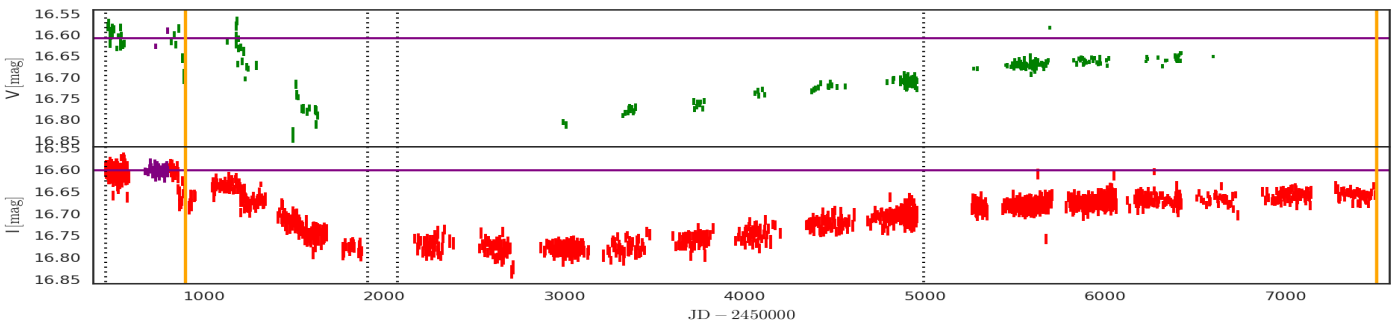

Figura 3.8: Idem à Fig. 3.7 para duas curvas de luz que apresentam bumps extremamente longos.

Por convenção, esta característica será referida como "Bumps extremamente longos", ou $\mathrm{VLB}^{8}$. Os VLBs possuem um rápido aumento (diminuição) de brilho devido ao surgimento de um disco. O retorno ao nível fotosférico prévio, é extremamente lento, indicando que o parâmetro de viscosidade $\alpha$ deve ser bem baixo. Os três eventos mais extensos têm durações de 7350,7325 e 7225 dias, aproximadamente. Adotamos, arbitrariamente, o valor de 4000 dias para definir um VLB, baseado na amostra de Rímulo et al. (2018), cujo evento de maior duração foi de 3300 dias. Em nossa amostra, temos 26 eventos VLB. Mesmo considerando essa definição arbitrária, destaca-se o grande número de eventos longos observados, o que pode sugerir um comportamento distinto dos discos estrelas Be da LMC em comparação aos da SMC.

\subsection{Descrição da natureza da amostra de curvas}

Passamos à caracterização das morfologias presentes na nossa amostra completa de curvas de luz. Quando necessário, utilizaremos os modos identificados por Keller et al. (2002) por conveniência.

Na Fig. 3.9 apresentamos as principais características morfológicas das curvas de luz que compõem a nossa amostra global. No painel A, observamos variações de curta duração

\footnotetext{
${ }^{8}$ Very Long Bumps
} 
e amplitudes aproximadamente constante. A morfologia desse tipo de evento se assemelha à características dos flickers identificados por Keller et al.: o evento é aproximadamente simétrico, no sentido que o aumento da magnitude possui aproximadamente a mesma duração da diminuição (em discrepância com os bumps). Muito provavelmente, esses eventos estão ligados à atividades curtas de perda de massa. Descendo para a imagem $\mathrm{B}$, temos uma morfologia que se equivale aos eventos degrau. Nesse caso, o brilho estelar tem uma mudança abrupta, atingindo um novo patamar. Caso a perda de massa continue existindo, o disco será mantido e o novo patamar de brilho se manterá aproximadamente constante. Muito provavelmente, esses são VLB que ainda não terminaram. No painel C, temos variações irregulares e de grande amplitude, não sendo possível definir um bump ou dip, ou mesmo estimar um nível fotosférico, pois este não é claramente identificável. Isso ocorre pela presença de um disco que, aparentemente, possui um esvanecimento temporário, seguido por uma retomada da perda de massa e, consequentemente, uma recuperação do brilho aparente.

No quadro D, observamos a presença de três características identificadas por Keller et al. simultaneamente: bumps, flickers e a impossibilidade de encontrar o nível fotosférico. Esse exemplo demonstra que os processos físicos que geram as diferentes características morfológicas podem estar simultaneamente presentes. Por outro lado, há também a ocorrência de pequenas variações em torno de um nível fotosférico aproximadamente constante, como o exibido pela estrela do painel E. Neste caso, pode-se tratar de uma estrela B normal, ou de uma Be que não apresentou evento de disco durante o período das observações. Há casos bastante curiosos, em que uma periodicidade ou quase-periodicidade pode ser notada visualmente, como a apresentada pela curva de luz mostrada na imagem H. Isso nos motivou a realizar um estudo inicial utilizando periodogramas que é apresentado na seção 3.5.1.

\subsubsection{Fenômenos periódicos e quase-periódicos}

Para melhor avaliar a aparente periodicidade de determinadas curvas de luz, fizemos uma análise temporal empregando o método desenvolvido por Lomb (1976) e Scargle (1982). O periodograma de Lomb-Scargle é uma ferramenta comum na análise de frequências de dados não igualmente espaçados e é equivalente a um ajuste de mínimos quadrados para uma onda senoidal da forma $y=a \cos \omega t+b \operatorname{sen} \omega t$. O estudo de periodi- 
A

LMC 162.8.29248

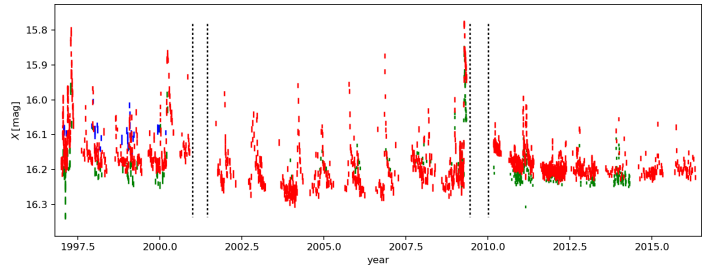

$\mathrm{B}$

LMC 100.1.75714

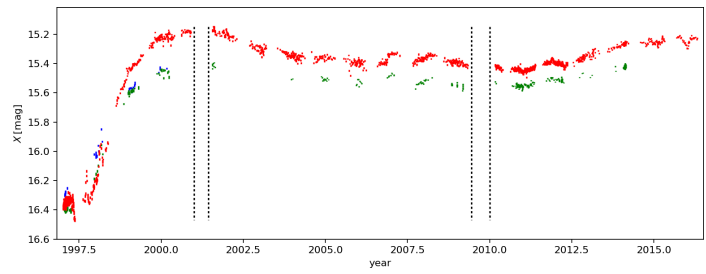

C

LM 169.4 .51316

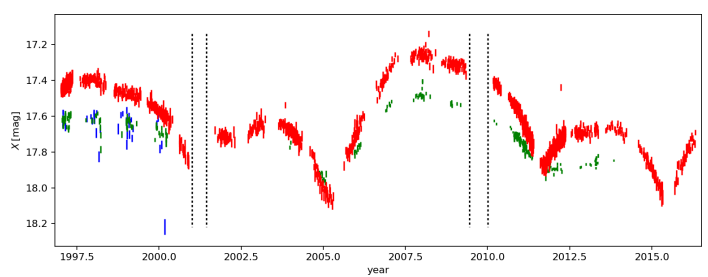

$\mathrm{D}$

LMC 170.2.60367

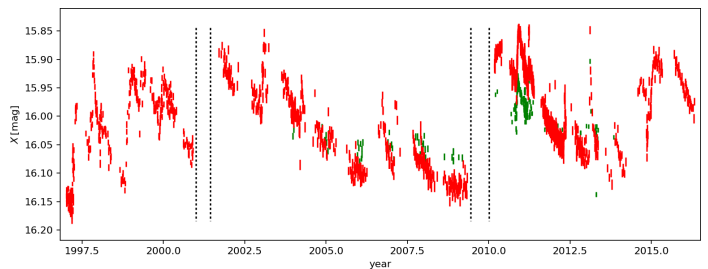

$\mathrm{E}$

LMC 168.8.192

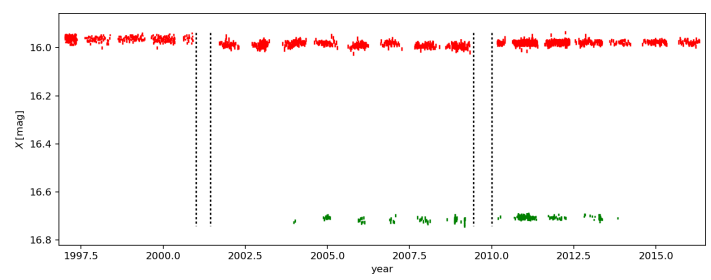

F

LMC 169.6.20754

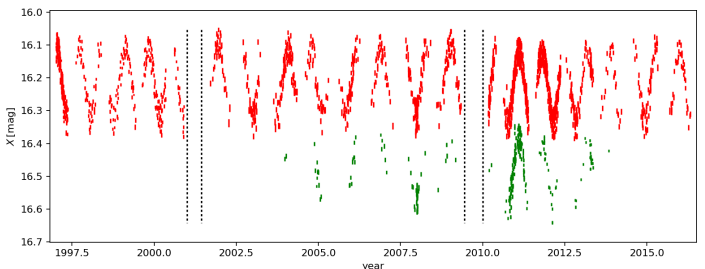

Figura 3.9: Exemplares de características morfológicas da amostra completa de curvas de luz.

cidade foi realizado para a amostra completa (contendo as duas mil curvas de luz).

Cada periodograma é composto da curva de luz analisada, na primeira linha, seguida da curva senoide para o período mais provável e a potência estatística em função do período (em dias) no eixo horizontal.

Na fig. 3.10 são apresentados alguns exemplos de periodogramas obtidos. Os resultados obtidos a partir dessa análise podem ser divididos em quatro casos, são eles:

\section{- Aperiódicos;}

- Quase-Periódicos: Curvas de luz que apresentam mais de um período (vide imagem superior esquerda da Fig. 3.10) ou um intervalo de períodos possíveis (Fig. 3.10, painel direito superior);

- Periódicos de curta duração ( $\lesssim 3$ dias): Possivelmente estão associados a fenômenos de pulsação estelar (imagem inferior esquerda da Fig. 3.10); 
- Periódicos de longa duração: Podem estar associadas à presença de uma binária não detectada ou por perturbações de densidade (veja Escolano et al. 2015 para mais detalhes).

No total, foram encontradas 112 estrelas periódicas (5,5\% da amostra) e 137 quaseperiódicas $(6,7 \%)$.

Para entendermos melhor a natureza dessa (quase-)periodicidade é necessário uma análise mais detalhada do que a realizada aqui, filtrando frequências ressonantes e de ruídos. Uma metodologia bastante robusta utilizada para esse tipo de estudo mais detalhado é a análise por wavelet, que é capaz de identificar evoluções temporais da variabilidade e a relação de fase entre supostos fenômenos de batimentos, que tipicamente não são visualizáveis em uma análise pelo método de Lomb-Scargle. Esta análise será parte do trabalho de doutorado do candidato.

\subsection{Morfologia de curvas de luz}

É importante ressaltar que as classificações propostas por Keller et al. (2002) e Mennickent et al. (2002) antecederam, em meia década, os primeiros estudos dinâmicos de discos de estrelas Be (Carciofi et al. 2012; Haubois et al. 2012). Referir-nos-emos aos modos identificados por Keller et al., mas a correspondência com os tipos de eventos indicados por Mennickent et al. pode ser facilmente realizada (o que foi feito na seção 3.2).

A morfologia das curvas de luz será controlada, basicamente, pela intensidade e variação da perda de massa, quando a estrela está em uma fase ativa; ou pela taxa de dissipação do disco, quando a estrela está inativa (sem perda de massa) e o disco se dissipa passivamente, através da viscosidade. Ejeções episódicas de massa de pequena intensidade provavelmente estão associadas ao eventos de flickers. Nesse cenário, a curva fotométrica correspondente possui forma aproximadamente simétrica, semelhante à fenomenologia observados no painel A da Fig. 3.9.

Perdas de massa mais intensas e espaçadas possivelmente estão associadas aos bumps (dips). Uma característica típica desses eventos é que o tempo de duração da dissipação é aproximadamente o dobro do tempo de construção do disco. O intervalo entre as injeções de matéria é suficientemente espaçado para que o disco seja completamente dissipado, i.e., o nível fotométrico retorna ao valor fotosférico. Vale notar que nos resultados teóricos de 
LMC 103.4.84579
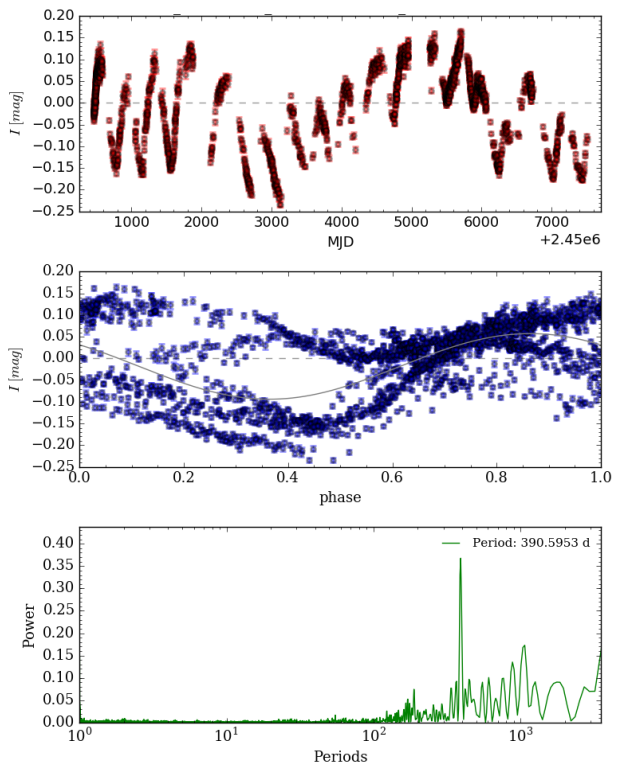

LMC 161.1.80259
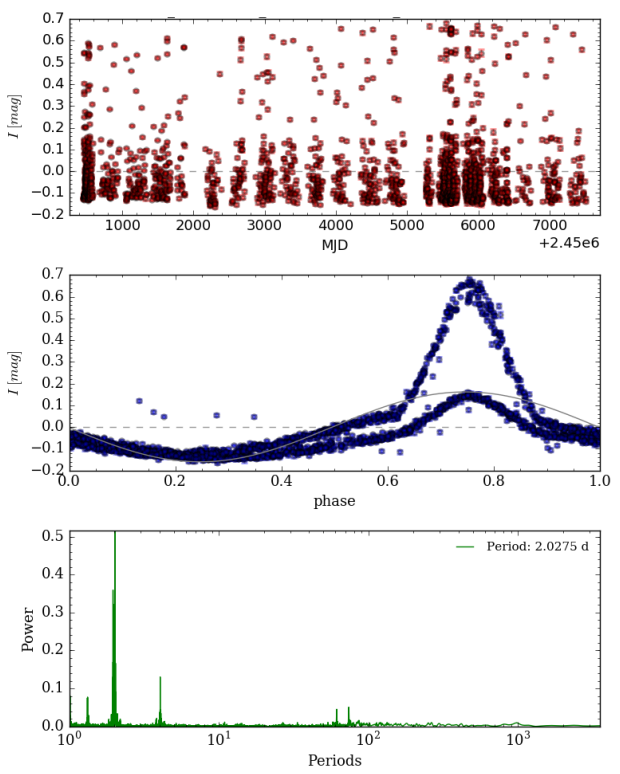

LMC 100.8.17896
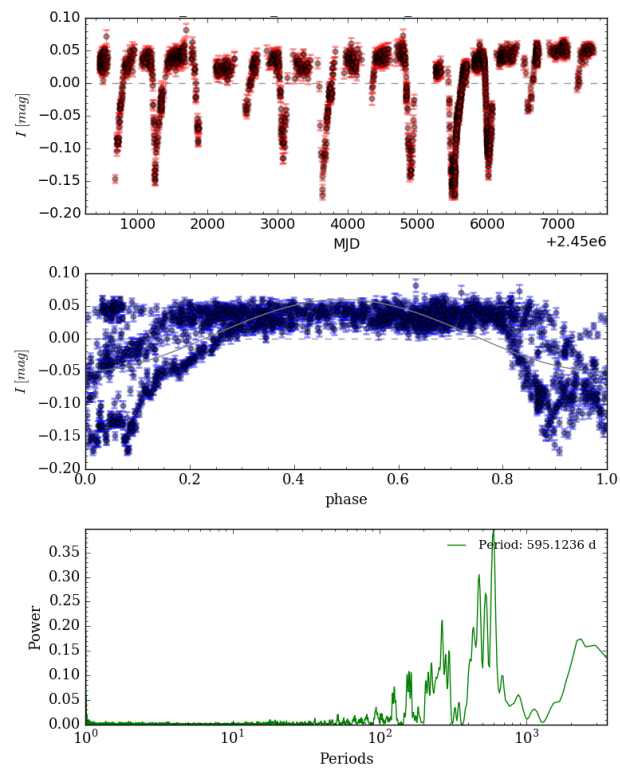

LMC103.3.78287
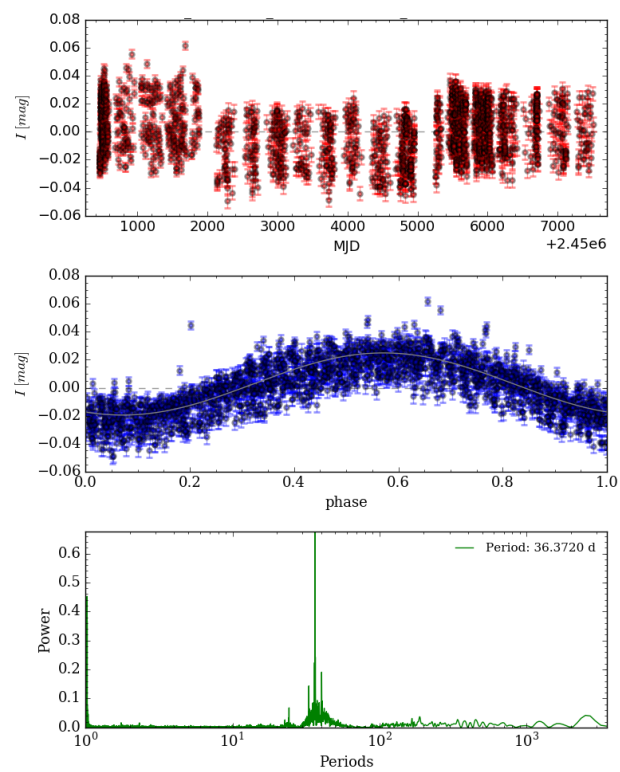

Figura 3.10: Periodogramas para estrelas quase periódicas (linha superior) e periódicas. Cada imagem é constituída por três painéis, que são compostos pela curva de luz, a senoide ajustada para o período mais provável encontrado e o gráfico da potencia estatística para diferentes períodos, respectivamente.

Haubois et al. (2012), a dissipação do disco é sempre mais lenta que a formação, de acordo, portanto, com as observações do OGLE.

É possível que existam estrelas intermediárias entre eventos de bumps e flickers, ou nas quais ambas morfologias possam coexistir, o que poderia explicar a quantidade abundante de dips observados na Fig. 3.7 e a presença de ambas fenomenologias no painel D da Fig. 
3.9.

Para o caso de VLBs, uma janela observacional temporalmente curta pode levar à observações de partes do fenômeno. Durante o aumento (diminuição) de brilho, a curva de luz pode ser classificada como um evento step, onde as variações fotométricas são abruptas e rápidas. Caso a observação ocorra durante a fase dissipativa, a morfologia pode ser classificada como um evento de variação de linha de base. Isso ressalta a grande importância da longa base temporal fornecida pelos levantamentos OGLE-II, III e IV combinados.

Os eventos de fading, podem ser interpretados como estrelas que possuem disco, e cuja taxa de injeção de massa cessa momentaneamente. Essa interrupção é rápida a ponto de o disco não se dissipar completamente, voltando a ser alimentado. A correspondência observacional para este cenário é uma variação do nível fotométrico, como o modo 5 observado na Fig. 3.1, e nos painéis B, C e F da Fig. 3.9.

Note que o efeito de reservatório de massa potencialmente está presente em todas as morfologias, exceto, possivelmente, nos flickers, devido à baixa quantidade de massa ejetada da estrela.

A partir das observações feitas acima, o estudo da periodicidade também constitui importante elemento para uma compreensão global do fenômeno Be e suas correspondências observacionais. Gostaríamos de ressaltar, que entre as perspectivas do trabalho atual, está o estudo mais detalhado e aprofundado da periodicidade, bem como as características gerais da amostra completa de curvas de luz (como amplitudes, tempos de construção, dissipação e de duração total dos eventos de disco), que aqui foram apresentados apenas para os eventos de disco selecionados, mas que será estendido para toda a amostra.

O estudo de amostra completa de curvas de luz de estrelas Be pode ter uma contribuição interessante ao quebra-cabeças que o fenômeno Be vem demonstrando ser. 
Tabela 3.2 - Estrelas Be e os respectivos bumps selecionados para este estudo.

\begin{tabular}{|c|c|c|c|c|c|c|c|c|c|c|}
\hline $\begin{array}{r}\mathrm{O} \\
\text { Campo }\end{array}$ & OGLE-III & \multicolumn{2}{|c|}{$\begin{array}{r}\text { Intervalo sem disco } \\
\text { (JD-2450000) }\end{array}$} & $B_{*}$ & $V_{*}$ & $I_{*}$ & \multirow{2}{*}{$\begin{array}{r}\text { Bump } \\
\text { ID } \\
01\end{array}$} & \multirow{2}{*}{$\begin{array}{r}\text { Bandas } \\
V I\end{array}$} & \multicolumn{2}{|c|}{$\begin{array}{r}\text { Intervalo do bump } \\
\text { (JD-2450000) }\end{array}$} \\
\hline LMC169.1 & 80968 & 2800 & 3200 & - & $15,986 \pm 0,006$ & $16,036 \pm 0,008$ & & & 3150 & 7000 \\
\hline LMC169.3 & 17823 & 1000 & 1350 & $18,082 \pm 0,02$ & $18,06 \pm 0,018$ & $17,964 \pm 0,033$ & 01 & $V I$ & 1450 & 7900 \\
\hline LMC168.8 & 78093 & 1000 & 1400 & $17,28 \pm 0,014$ & $17,486 \pm 0,013$ & $17,564 \pm 0,024$ & 01 & $V I$ & 1350 & 4000 \\
\hline LMC169.8 & 56222 & 6000 & 6500 & - & $16,734 \pm 0,005$ & $16,768 \pm 0,011$ & 01 & $I$ & 6550 & 7900 \\
\hline LMC169.7 & 64117 & 600 & 800 & $17,576 \pm 0,017$ & $17,733 \pm 0,014$ & $17,743 \pm 0,03$ & $\begin{array}{l}01 \\
02\end{array}$ & $\begin{array}{l}V I \\
V I\end{array}$ & $\begin{array}{c}800 \\
3900\end{array}$ & $\begin{array}{l}3000 \\
4900\end{array}$ \\
\hline LMC169.3 & 338 & 1500 & 1700 & $16,853 \pm 0,013$ & $16,917 \pm 0,012$ & $16,946 \pm 0,013$ & $\begin{array}{l}01 \\
02 \\
03\end{array}$ & $\begin{array}{r}V I \\
V I \\
\\
I\end{array}$ & $\begin{array}{l}1875 \\
3750 \\
5000\end{array}$ & $\begin{array}{l}3500 \\
5000 \\
7000\end{array}$ \\
\hline LMC161.1 & 29883 & 4000 & 5000 & - & $17,522 \pm 0,01$ & $17,471 \pm 0,019$ & 01 & $V I$ & 4975 & 8000 \\
\hline LMC162.1 & 30252 & 400 & 550 & $16,89 \pm 0,011$ & $17,019 \pm 0,011$ & $17,071 \pm 0,012$ & 01 & $V I$ & 500 & 7300 \\
\hline LMC162.3 & 63945 & 3500 & 4000 & - & $17,119 \pm 0,008$ & $17,121 \pm 0,012$ & 01 & $V I$ & 4300 & 8000 \\
\hline LMC161.2 & 38040 & 4500 & 5000 & - & $16,009 \pm 0,005$ & $16,056 \pm 0,008$ & 01 & $V I$ & 5150 & 8000 \\
\hline LMC162.2 & 38930 & 650 & 800 & $16,586 \pm 0,01$ & $16,605 \pm 0,007$ & $16,598 \pm 0,01$ & 01 & $V I$ & 950 & 8000 \\
\hline LMC169.7 & 103494 & 3800 & 4150 & - & $17,391 \pm 0,01$ & $17,472 \pm 0,017$ & $\begin{array}{l}01 \\
02\end{array}$ & $\begin{array}{r}I \\
V I\end{array}$ & $\begin{array}{l}2700 \\
4200\end{array}$ & $\begin{array}{l}4000 \\
5000\end{array}$ \\
\hline
\end{tabular}


Tabela 3.2 - continuação da página anterior

\begin{tabular}{|c|c|c|c|c|c|c|c|c|c|c|}
\hline & & & & & & & $\begin{array}{l}03 \\
04\end{array}$ & $\begin{array}{r}V I \\
I\end{array}$ & $\begin{array}{l}5200 \\
7000\end{array}$ & $\begin{array}{l}6800 \\
7500\end{array}$ \\
\hline LMC169.6 & 80441 & 400 & 1000 & $16,72 \pm 0,012$ & $16,86 \pm 0,009$ & $16,692 \pm 0,01$ & 01 & $V I$ & 1200 & 3000 \\
\hline LMC162.8 & 106499 & 5000 & 5550 & - & $16,953 \pm 0,006$ & $16,896 \pm 0,012$ & 01 & $V I$ & 5550 & 7600 \\
\hline LMC162.2 & 76010 & 5700 & 5900 & - & $17,044 \pm 0,007$ & $16,96 \pm 0,013$ & 01 & $V I$ & 6100 & 8000 \\
\hline LMC162.3 & 22539 & 600 & 1000 & $16,881 \pm 0,011$ & $16,946 \pm 0,01$ & $16,967 \pm 0,014$ & 01 & $V I$ & 900 & 7600 \\
\hline LMC162.3 & 22483 & 5400 & 5700 & - & $16,492 \pm 0,005$ & $16,549 \pm 0,01$ & 01 & $V I$ & 5930 & 8000 \\
\hline LMC162.6 & 62005 & 400 & 1100 & $16,652 \pm 0,012$ & $16,805 \pm 0,01$ & $16,897 \pm 0,013$ & 01 & $V I$ & 1400 & 4300 \\
\hline LMC161.8 & 51737 & 400 & 650 & $16,081 \pm 0,008$ & $16,185 \pm 0,008$ & $16,243 \pm 0,007$ & 01 & $V I$ & 850 & 2000 \\
\hline LMC162.6 & 101791 & 3500 & 3750 & - & $16,145 \pm 0,006$ & $16,247 \pm 0,008$ & 01 & $V I$ & 3850 & 8000 \\
\hline LMC161.8 & 18912 & 600 & 900 & $16,582 \pm 0,011$ & $16,657 \pm 0,01$ & $16,713 \pm 0,013$ & $\begin{array}{l}01 \\
02\end{array}$ & $\begin{array}{l}V I \\
V I\end{array}$ & $\begin{array}{c}975 \\
4450\end{array}$ & $\begin{array}{l}3500 \\
8000\end{array}$ \\
\hline LMC162.7 & 108765 & 400 & 600 & $16,343 \pm 0,009$ & $16,469 \pm 0,009$ & $16,464 \pm 0,008$ & 01 & $V I$ & 600 & 8000 \\
\hline LMC161.8 & 35769 & 4000 & 4500 & - & $16,893 \pm 0,007$ & $16,945 \pm 0,013$ & 01 & $V I$ & 4650 & 8000 \\
\hline LMC161.8 & 99532 & 600 & 900 & $17,21 \pm 0,013$ & $17,306 \pm 0,012$ & $17,355 \pm 0,022$ & 01 & $V I$ & 850 & 5000 \\
\hline LMC162.6 & 82373 & 4300 & 4550 & - & $16,66 \pm 0,006$ & $16,679 \pm 0,011$ & 01 & $V I$ & 4650 & 8000 \\
\hline LMC161.7 & 45493 & 600 & 1300 & - & - & $17,596 \pm 0,015$ & 01 & $I$ & 1300 & 8000 \\
\hline LMC162.5 & 748 & 1300 & 1500 & - & $16,724 \pm 0,013$ & $16,86 \pm 0,015$ & $\begin{array}{l}01 \\
02\end{array}$ & $\begin{array}{l}V I \\
V I\end{array}$ & $\begin{array}{l}1400 \\
3700\end{array}$ & $\begin{array}{l}3800 \\
4900\end{array}$ \\
\hline
\end{tabular}

Continua na próxima página 
Tabela 3.2 - continuação da página anterior

\begin{tabular}{|c|c|c|c|c|c|c|c|c|c|c|}
\hline & & & & & & & 03 & $V I$ & 6000 & 8000 \\
\hline LMC161.8 & 1506 & 600 & 1200 & $17,509 \pm 0,018$ & $17,576 \pm 0,014$ & $17,75 \pm 0,024$ & 01 & $V I$ & 1200 & 8000 \\
\hline \multirow[t]{2}{*}{ LMC161.8 } & 84095 & 400 & 590 & $16,179 \pm 0,009$ & $16,324 \pm 0,008$ & $16,361 \pm 0,008$ & 01 & $V I$ & 600 & 3500 \\
\hline & & & & & & & 02 & $V I$ & 3400 & 5800 \\
\hline LMC100.2 & 89063 & 600 & 1400 & $16,285 \pm 0,011$ & $16,394 \pm 0,01$ & $16,501 \pm 0,011$ & 01 & $V I$ & 1650 & 3000 \\
\hline \multirow[t]{3}{*}{ LMC103.3 } & 78875 & 5400 & 5560 & - & $16,718 \pm 0,005$ & $16,782 \pm 0,009$ & 01 & $I$ & 1700 & 3900 \\
\hline & & & & & & & 02 & $V I$ & 3975 & 5500 \\
\hline & & & & & & & 03 & $V I$ & 5575 & 6500 \\
\hline LMC100.1 & 133871 & 2100 & 2300 & - & $14,325 \pm 0,004$ & $14,44 \pm 0,005$ & 01 & $V I$ & 2450 & 5000 \\
\hline \multirow[t]{4}{*}{ LMC100.1 } & 95208 & 2000 & 2400 & - & $16,262 \pm 0,006$ & $16,346 \pm 0,009$ & 01 & $V I$ & 400 & 1200 \\
\hline & & & & & & & 02 & $V I$ & 2500 & 3500 \\
\hline & & & & & & & 03 & $V I$ & 4150 & 5300 \\
\hline & & & & & & & 04 & $V I$ & 5450 & 6600 \\
\hline \multirow[t]{3}{*}{ LMC100.8 } & 129218 & 3300 & 3450 & - & $15,463 \pm 0,005$ & $15,265 \pm 0,006$ & 01 & $V I$ & 700 & 2400 \\
\hline & & & & & & & 02 & $V I$ & 3525 & 4500 \\
\hline & & & & & & & 03 & $V I$ & 5900 & 7500 \\
\hline LMC100.6 & 50481 & 400 & 600 & $15,195 \pm 0,007$ & $15,368 \pm 0,007$ & $15,529 \pm 0,005$ & 01 & $V I$ & 700 & 2000 \\
\hline LMC100.6 & 34598 & 2000 & 3200 & - & $16,805 \pm 0,008$ & $16,614 \pm 0,011$ & 01 & $V I$ & 3325 & 5000 \\
\hline LMC100.1 & 1702 & 800 & 1400 & $17,524 \pm 0,017$ & $17,624 \pm 0,014$ & $17,696 \pm 0,029$ & 01 & $V I$ & 1400 & 8000 \\
\hline
\end{tabular}


Tabela 3.2 - continuação da página anterior

\begin{tabular}{|c|c|c|c|c|c|c|c|c|c|c|}
\hline LMC100.8 & 91619 & 1000 & 1500 & $16,369 \pm 0,013$ & $16,495 \pm 0,01$ & $16,513 \pm 0,013$ & $\begin{array}{l}01 \\
02\end{array}$ & $\begin{array}{l}V I \\
V I\end{array}$ & $\begin{array}{l}1500 \\
5600\end{array}$ & $\begin{array}{l}5000 \\
8000\end{array}$ \\
\hline LMC100.8 & 91630 & 400 & 500 & - & - & $16,269 \pm 0,007$ & 01 & $V I$ & 400 & 5000 \\
\hline LMC103.5 & 76737 & 6050 & 6300 & - & $15,996 \pm 0,004$ & $15,802 \pm 0,006$ & $\begin{array}{l}01 \\
02\end{array}$ & $\begin{array}{r}V I \\
\quad I\end{array}$ & $\begin{array}{l}3000 \\
6500\end{array}$ & $\begin{array}{l}4200 \\
8000\end{array}$ \\
\hline LMC100.8 & 19248 & 600 & 1150 & $17,893 \pm 0,02$ & $17,853 \pm 0,017$ & $17,92 \pm 0,03$ & 01 & $V I$ & 1000 & 7000 \\
\hline LMC100.7 & 34608 & 400 & 1300 & $16,365 \pm 0,014$ & $16,416 \pm 0,011$ & $16,421 \pm 0,01$ & 01 & $V I$ & 1300 & 6200 \\
\hline LMC100.7 & 18811 & 400 & 600 & $18,343 \pm 0,046$ & $18,32 \pm 0,018$ & $18,239 \pm 0,034$ & 01 & $V I$ & 600 & 8000 \\
\hline LMC111.2 & 37142 & 2100 & 2700 & - & - & $17,166 \pm 0,014$ & 01 & $I$ & 2700 & 6000 \\
\hline LMC111.2 & 87298 & 400 & 600 & $16,444 \pm 0,011$ & $16,504 \pm 0,009$ & $16,595 \pm 0,009$ & $\begin{array}{l}01 \\
02\end{array}$ & $\begin{array}{l}V I \\
V I\end{array}$ & $\begin{array}{c}640 \\
6180\end{array}$ & $\begin{array}{l}1100 \\
7000\end{array}$ \\
\hline LMC111.3 & 61518 & 400 & 1300 & $17,869 \pm 0,02$ & $17,768 \pm 0,015$ & $17,831 \pm 0,026$ & 01 & $V I$ & 1350 & 8000 \\
\hline LMC111.2 & 74618 & 5500 & 5800 & - & $17,134 \pm 0,007$ & $17,193 \pm 0,012$ & 02 & $V I$ & 5750 & 7000 \\
\hline LMC111.3 & 23404 & 3250 & 3430 & - & $16,709 \pm 0,008$ & $16,726 \pm 0,01$ & 01 & $V I$ & 3400 & 8000 \\
\hline LMC111.7 & 70752 & 400 & 850 & $15,808 \pm 0,035$ & $16,621 \pm 0,01$ & $16,432 \pm 0,009$ & $\begin{array}{l}01 \\
02\end{array}$ & $\begin{array}{l}I \\
I\end{array}$ & $\begin{array}{l}1700 \\
6350\end{array}$ & $\begin{array}{l}3500 \\
8000\end{array}$ \\
\hline LMC111.3 & 50562 & 400 & 600 & - & $16,543 \pm 0,008$ & $16,694 \pm 0,008$ & 01 & $V I$ & 600 & 3800 \\
\hline LMC110.8 & 74753 & 400 & 600 & - & $14,762 \pm 0,008$ & $14,97 \pm 0,004$ & 01 & $V I$ & 1200 & 4000 \\
\hline LMC111.6 & 31665 & 2600 & 2800 & - & - & $16,714 \pm 0,012$ & 01 & $V I$ & 2600 & 5000 \\
\hline
\end{tabular}

Continua na próxima página 
Tabela 3.2 - continuação da página anterior

\begin{tabular}{|c|c|c|c|c|c|c|c|c|c|c|}
\hline & & & & & & & 02 & $V I$ & 5300 & 7000 \\
\hline LMC111.7 & 50820 & 5200 & 6000 & - & $16,403 \pm 0,004$ & $16,38 \pm 0,007$ & 01 & $V I$ & 1800 & 5000 \\
\hline LMC111.5 & 53741 & 4500 & 5000 & - & $16,846 \pm 0,007$ & $16,859 \pm 0,011$ & 01 & $V I$ & 400 & 3500 \\
\hline \multirow[t]{2}{*}{ LMC119.4 } & 80248 & 600 & 1000 & $15,674 \pm 0,007$ & $15,72 \pm 0,008$ & $15,671 \pm 0,006$ & 01 & $I$ & 1450 & 4000 \\
\hline & & & & & & & 02 & $I$ & 3900 & 8000 \\
\hline \multirow[t]{2}{*}{ LMC118.2 } & 27272 & 4500 & 5000 & - & $16,921 \pm 0,009$ & $16,929 \pm 0,013$ & 01 & $V I$ & 2750 & 5000 \\
\hline & & & & & & & 02 & $V I$ & 4900 & 8000 \\
\hline \multirow[t]{2}{*}{ LMC118.2 } & 83574 & 4000 & 4400 & - & $16,52 \pm 0,006$ & $16,486 \pm 0,009$ & 01 & $V I$ & 600 & 4000 \\
\hline & & & & & & & 02 & $I$ & 4300 & 8000 \\
\hline LMC119.2 & 89739 & 600 & 1000 & $17,044 \pm 0,018$ & $17,058 \pm 0,013$ & $17,081 \pm 0,014$ & 01 & $V I$ & 1000 & 5000 \\
\hline \multirow[t]{2}{*}{ LMC119.3 } & 41808 & 2500 & 2800 & - & - & $16,293 \pm 0,008$ & 01 & $I$ & 2900 & 5000 \\
\hline & & & & & & & 02 & $I$ & 6750 & 8000 \\
\hline LMC111.7 & 42828 & 400 & 850 & $17,852 \pm 0,015$ & $17,889 \pm 0,017$ & $17,953 \pm 0,025$ & 01 & $V I$ & 800 & 5000 \\
\hline \multirow[t]{2}{*}{ LMC110.8 } & 117 & 3550 & 3650 & - & - & $15,501 \pm 0,006$ & 01 & $I$ & 600 & 3500 \\
\hline & & & & & & & 02 & $V I$ & 3650 & 6000 \\
\hline \multirow[t]{2}{*}{ LMC119.7 } & 92903 & 2950 & 3000 & - & $15,929 \pm 0,005$ & $15,967 \pm 0,007$ & 01 & $V I$ & 3000 & 5000 \\
\hline & & & & & & & 02 & $V I$ & 5350 & 7000 \\
\hline \multirow[t]{2}{*}{ LMC120.1 } & 40130 & 1500 & 1520 & $15,559 \pm 0,008$ & $15,662 \pm 0,006$ & $15,594 \pm 0,007$ & 01 & $I$ & 1500 & 2800 \\
\hline & & & & & & & 02 & $I$ & 2980 & 3700 \\
\hline
\end{tabular}


Tabela 3.2 - continuação da página anterior

\begin{tabular}{|c|c|c|c|c|c|c|c|c|c|c|}
\hline & & & & & & & 03 & $V I$ & 5350 & 5800 \\
\hline \multirow[t]{2}{*}{ LMC120.1 } & \multirow[t]{2}{*}{40173} & \multirow[t]{2}{*}{400} & \multirow[t]{2}{*}{600} & \multirow{2}{*}{\multicolumn{2}{|c|}{$-\quad 16,282 \pm 0,009$}} & \multirow[t]{2}{*}{$16,296 \pm 0,007$} & 01 & $V I$ & 600 & 2500 \\
\hline & & & & & & & 02 & $V I$ & 4200 & 7000 \\
\hline LMC118.5 & 53191 & 1000 & 1550 & $17,518 \pm 0,016$ & $17,579 \pm 0,016$ & $17,511 \pm 0,025$ & 01 & $V I$ & 1550 & 8000 \\
\hline \multirow[t]{2}{*}{ LMC118.2 } & \multirow[t]{2}{*}{56865} & \multirow[t]{2}{*}{1000} & \multirow[t]{2}{*}{1350} & \multirow[t]{2}{*}{$16,786 \pm 0,012$} & \multirow[t]{2}{*}{$16,86 \pm 0,011$} & \multirow[t]{2}{*}{$16,928 \pm 0,012$} & 01 & $I$ & 1350 & 3000 \\
\hline & & & & & & & 02 & $V I$ & 6150 & 7500 \\
\hline LMC118.5 & 23216 & 900 & 1000 & - & - & $16,482 \pm 0,012$ & 01 & $V I$ & 950 & 8000 \\
\hline LMC119.3 & 69304 & 2000 & 3000 & - & $17,194 \pm 0,008$ & $17,208 \pm 0,015$ & 01 & $V I$ & 3250 & 8000 \\
\hline \multirow[t]{2}{*}{ LMC119.4 } & \multirow[t]{2}{*}{14199} & \multirow[t]{2}{*}{400} & \multirow[t]{2}{*}{900} & \multirow[t]{2}{*}{$17,268 \pm 0,012$} & \multirow[t]{2}{*}{$17,274 \pm 0,013$} & \multirow[t]{2}{*}{$17,144 \pm 0,014$} & 01 & $V I$ & 800 & 4000 \\
\hline & & & & & & & 02 & $V I$ & 4900 & 8000 \\
\hline LMC119.6 & 66150 & 2000 & 2500 & - & - & $16,527 \pm 0,009$ & 01 & $I$ & 3100 & 8000 \\
\hline LMC118.8 & 62707 & 1000 & 1350 & $16,979 \pm 0,017$ & $16,979 \pm 0,013$ & $16,926 \pm 0,013$ & 01 & $V I$ & 1320 & 8000 \\
\hline LMC119.7 & 66528 & 3900 & 4250 & - & $17,161 \pm 0,008$ & $17,283 \pm 0,015$ & 01 & $V I$ & 4500 & 8000 \\
\hline LMC119.6 & 66163 & 1000 & 1400 & $17,502 \pm 0,015$ & $17,516 \pm 0,015$ & $17,48 \pm 0,02$ & 01 & $V I$ & 1400 & 5000 \\
\hline LMC119.5 & 13208 & 5200 & 5300 & - & $16,614 \pm 0,005$ & $16,621 \pm 0,009$ & 01 & $V I$ & 5250 & 8000 \\
\hline \multirow[t]{2}{*}{ LMC127.2 } & \multirow[t]{2}{*}{18027} & \multirow[t]{2}{*}{2900} & \multirow[t]{2}{*}{3200} & \multirow[t]{2}{*}{ - } & \multirow[t]{2}{*}{$14,982 \pm 0,004$} & \multirow[t]{2}{*}{$14,89 \pm 0,006$} & 01 & $V I$ & 3400 & 5000 \\
\hline & & & & & & & 02 & $V I$ & 5800 & 7400 \\
\hline LMC126.1 & 48828 & 1000 & 1500 & $16,204 \pm 0,012$ & $16,223 \pm 0,01$ & $16,27 \pm 0,008$ & 01 & $I$ & 1850 & 6000 \\
\hline LMC127.1 & 38725 & 3600 & 3950 & - & $15,459 \pm 0,004$ & $15,35 \pm 0,005$ & 01 & $V I$ & 4300 & 8000 \\
\hline
\end{tabular}

Continua na próxima página 
Tabela 3.2 - continuação da página anterior

\begin{tabular}{|c|c|c|c|c|c|c|c|c|c|c|}
\hline LMC127.4 & 10051 & 1100 & 1300 & $17,542 \pm 0,015$ & $17,602 \pm 0,016$ & $17,485 \pm 0,019$ & 01 & $V I$ & 1300 & 7000 \\
\hline LMC170.3 & 43578 & 600 & 950 & $17,132 \pm 0,013$ & $17,201 \pm 0,012$ & $17,371 \pm 0,016$ & 01 & $V I$ & 980 & 6000 \\
\hline LMC177.5 & 17601 & 600 & 1000 & $16,643 \pm 0,011$ & $16,611 \pm 0,008$ & $16,623 \pm 0,01$ & 01 & $V I$ & 900 & 8000 \\
\hline LMC176.6 & 30752 & 1500 & 1600 & $16,589 \pm 0,026$ & $16,681 \pm 0,009$ & $16,525 \pm 0,011$ & $\begin{array}{l}01 \\
02 \\
03 \\
04 \\
05\end{array}$ & $\begin{array}{r}I \\
V I \\
V I \\
V I \\
\quad I\end{array}$ & $\begin{array}{l}1600 \\
3340 \\
4525 \\
5800 \\
6970\end{array}$ & $\begin{array}{l}2700 \\
4550 \\
5750 \\
6850 \\
8400\end{array}$ \\
\hline LMC176.8 & 82613 & 4300 & 4600 & - & $17,905 \pm 0,012$ & $17,859 \pm 0,027$ & 01 & $V I$ & 4700 & 6400 \\
\hline LMC177.3 & 50735 & 2000 & 2250 & - & - & $16,794 \pm 0,011$ & $\begin{array}{l}01 \\
02 \\
03\end{array}$ & $\begin{array}{r}I \\
V I \\
V I\end{array}$ & $\begin{array}{l}2100 \\
3900 \\
5500\end{array}$ & $\begin{array}{l}3900 \\
5500 \\
8000\end{array}$ \\
\hline LMC185.8 & 314 & 4200 & 4600 & - & $17,418 \pm 0,01$ & $17,433 \pm 0,019$ & 01 & $V I$ & 4550 & 8000 \\
\hline LMC185.5 & 241 & 600 & 750 & - & - & $17,667 \pm 0,019$ & $\begin{array}{l}01 \\
02\end{array}$ & $\begin{array}{l}I \\
I\end{array}$ & $\begin{array}{r}780 \\
4900\end{array}$ & $\begin{array}{l}3000 \\
8000\end{array}$ \\
\hline
\end{tabular}


Capítulo 4

\section{Resultados}

\subsection{A influência da rotação nas curvas de luz}

Um efeito que não foi considerado por Rímulo et al. (2018) em sua modelagem é a influência do efeito da rotação e da idade da estrela na amplitude dos bumps. A rotação altera o campo radiativo da estrela (mais quente nos polos e mais frio no equador) bem como expande as regiões equatoriais. Aliada à rotação, a idade estelar modifica os valores de fluxo superficial, de raio (vide Fig. 2.6) e de rotação (Georgy et al., 2013). O resultado combinado de ambos efeitos é a alteração dos parâmetros estelares (como $R, W$ e $L$ ) e, consequentemente, das características do disco (como sua velocidade de rotação, escala de altura e temperatura).

Visando aprimorar a modelagem das curvas de luz, elaboramos uma grade de modelos alternativa para explorar os efeitos destas duas grandezas. Os parâmetros da grade estão listados na tabela 4.1. Computamos o fluxo emergente para duas situações distintas: estrelas sem disco e estrelas com discos em estado estacionário, ou seja, cujo tempo de construção é infinitamente grande. Dessa forma podemos avaliar o impacto máximo dos efeitos da rotação e idade na variação fotométrica observada.

Apresentamos como a idade e a rotação estelar afetam a amplitude máxima do bump para estrelas de 7 e $15 M_{\odot}$ para as bandas $I$ (Fig. 4.1) e $V$ (Fig. 4.2). É interessante notar que tanto o aumento da idade (e portanto do raio estelar) quando o aumento da rotação (que também resulta num maior raio do equador) possuem efeito de diminuir a amplitude do bump. Em ambos os casos esse efeito é mais pronunciado para a estrelas de $M=7 M_{\odot}$. A banda fotométrica $I$ é mais sensível a esses efeitos, se comparada à $V$.

Os resultados explicam-se por um efeito na densidade volumétrica. Como em todas 
Tabela 4.1 - Parâmetros para a grade de modelos estacionários.

\begin{tabular}{cccc}
\hline$Z$ & $M\left[M_{\odot}\right]$ & $t / t_{\mathrm{ms}}$ & $W$ \\
\hline 0,006 & 7 & 0,00 & 0,40 \\
& 11 & 0,50 & 0,60 \\
& 15 & 0,98 & 0,70 \\
& & & 0,80 \\
& & & 0,90 \\
& & & 0,95 \\
\hline
\end{tabular}
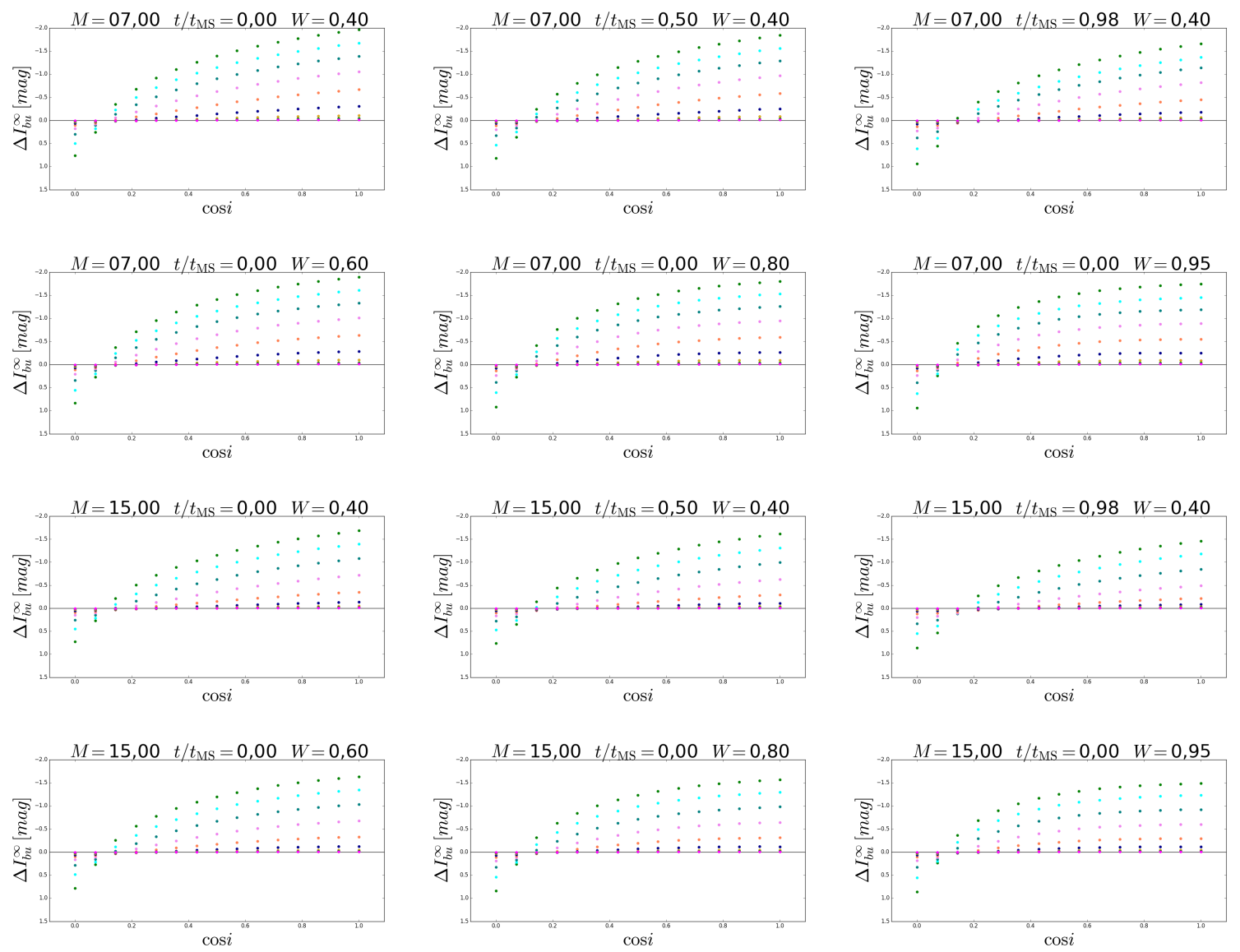

Figura 4.1: Amplitude máxima do bump na banda $I$, em função do ângulo do observador (cos $i$ ), observado para estrelas de $7 M_{\odot}$ (duas linhas de cima) e $15 M_{\odot}$ (duas linhas de baixo). Para cada massa, apresentamos os efeitos da idade $\left(t / t_{\mathrm{ms}}=0,00,0,50\right.$ e 0,98), na primeira e terceira linhas, e da rotação $(W=0,6,0,8$, 0,95), na segunda e quarta linhas. Os pontos de cores verde, azuis claro e escuro, rosa, laranja, azul, amarelo, verde e magenta representam densidades de $\Sigma_{0}=7,00,3,50,1,75,0,88,0,44,0,22,0,11,0,06$, 0,03 , respectivamente.

as simulações o $\Sigma_{0}$ foi mantido constante, o aumento do raio do equador implica numa maior escala de altura do disco e portanto numa menor densidade volumétrica. Como a emissão do disco escala basicamente com a densidade ao quadrado (processos livre-livre e 

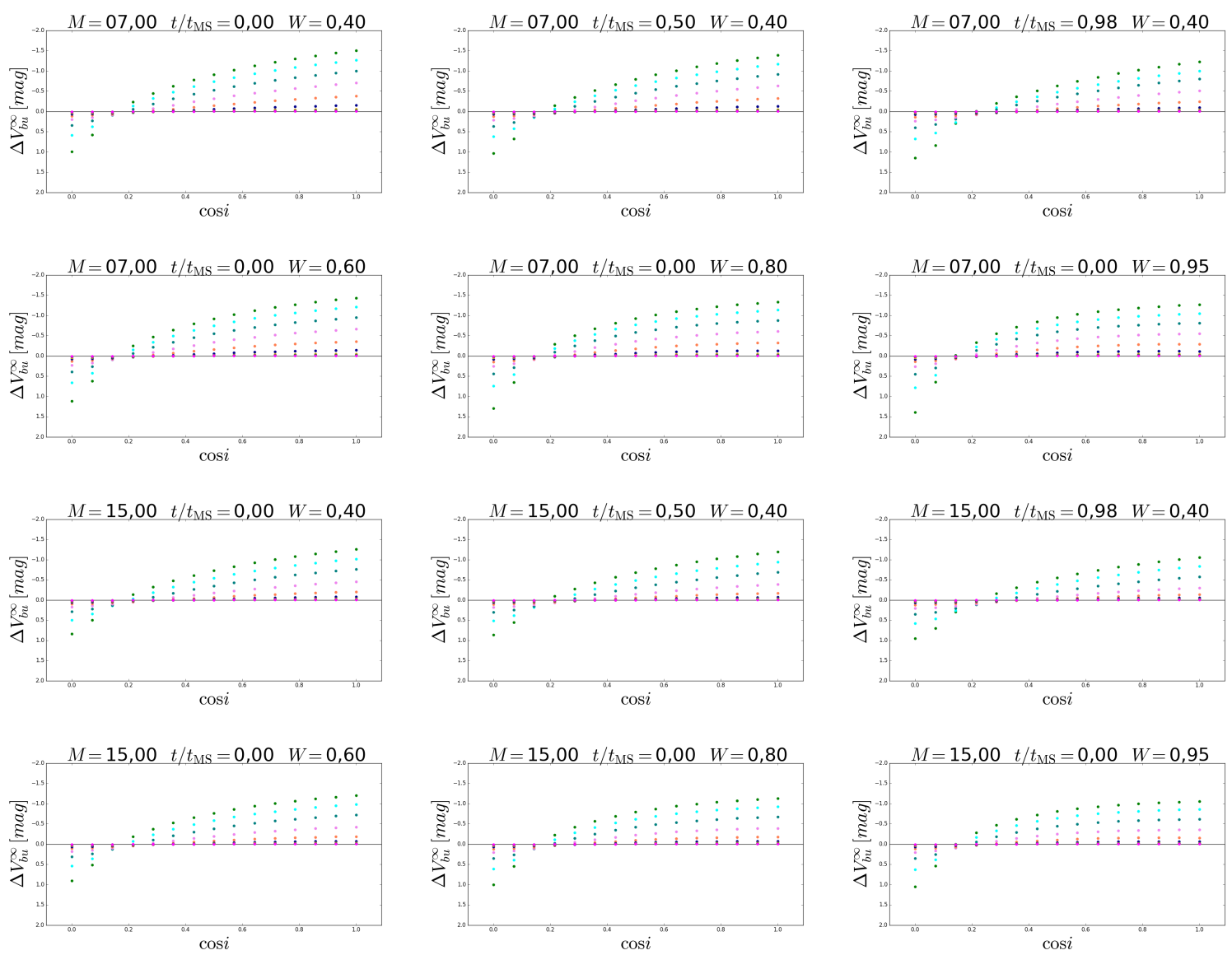

Figura 4.2: Idem à Fig. 4.1 para a banda fotométrica $V$.

livre-ligado), a emissão diminui.

Estes resultados mostram que é importante incluir, na grade de modelos de curvas de luz, os parâmetros idade e $W$. Esta é uma das perspectivas futuras deste trabalho (ver capítulo 6).

\subsection{Modelagem das estrelas LMC 162.7.108765 e LMC 127.1.38725}

Para ilustrar os resultados obtidos a partir das modelagens das curvas de luz da LMC selecionadas para este estudo, escolhemos duas estrelas, uma com dip (LMC 127.1.38725) e outra com bump (LMC 162.7.108765).

O resultado da modelagem destas estrelas é apresentado nas Figs. 4.3 e $4.4^{1}$. Os modelos utilizados para o presente estudo correspondem à mesma grade computada por Rímulo et al. (2018) para metalicidades da SMC. O cômputo de uma grade apropriada

\footnotetext{
${ }^{1}$ Embora figura semelhante tenha sido apresentada anteriormente (veja Fig. 2.8), por conveniência, novamente apresentamos a legenda.
} 
para a LMC é um esforço atualmente em andamento, que infelizmente não foi concluído a tempo para esta dissertação. Desta forma, os resultados aqui apresentados podem sofrer variações sistemáticas. Os parâmetros modelados para cada uma das estrelas podem ser encontrados na tabela 4.2 .

A pipeline ajusta o tempo de início da construção do disco, $t_{1}$, e do início da dissipação, $t_{2}$. Mas uma estimativa dessas quantidades é fornecida ao emcee por inspeção visual das curvas de luz, que são apresentadas como linhas horizontais roxa e amarela (respectivamente). Como explicado anteriormente na seção 2.4.1, a amostragem por MCMC, após um número suficiente de passos, atinge uma amostra estacionária. Nesse ponto, os parâmetros do modelos se concentram em regiões de maior probabilidade. As linhas verdes (banda $V$ ) e vermelha (banda $I$ ) são 100 modelos aleatoriamente sorteados da amostragem estacionária e podem ser utilizados como uma estimativa visual da qualidade do ajuste. Outra forma de avaliar a qualidade do ajuste, é através dos gráficos de probabilidades posteriores dos parâmetros ajustados nas Figs. 4.3 e 4.4. A diagonal principal do diagrama triangular exibe a distribuição dos parâmetros estelares $\left(M, t / t_{\mathrm{ms}}\right.$ e $\left.W\right)$, geométrico $(\cos i)$ e do bump $\left(\Sigma_{0}, \alpha_{\mathrm{bu}}\right.$ e $\left.\alpha_{\mathrm{d}}\right)$. As imagens abaixo da diagonal principal mostram as correlações entre os parâmetros.

No caso específico de LMC 127.1.38725 (Fig. 4.3), a ausência de dados próximos à $t_{2}$ dificultou a análise, aumentando a incerteza com relação a esse parâmetro. Um reflexo disso é o espalhamento dos modelos nas proximidades de $t_{2}$. A posição de LMC 127.1.38725 no diagrama de cor-magnitude mostra que essa está mais à direita do que o esperado para uma estrela na Sequência Principal. Como discutido anteriormente (vide seção 3.4), muito provavelmente isso é efeito de considerar um avermelhamento maior do que o real. Outro reflexo disso pode ser observado nos parâmetros estelares obtidos $\left(M, t / t_{\mathrm{ms}}\right.$ e $\left.W\right)$ que são restringidos pelas magnitudes na fase inativa. Os três primeiros painéis da diagonal principal apresentam distribuições alargadas, o que indica que esses parâmetros não foram bem caracterizados. O primeiro histograma mostra que LMC 127.1.38725 é uma estrela recente, cuja massa foi determinada em $M=17,5_{-4,1}^{+2,5} M_{\odot}$, o que concorda com a posição vertical da estrela no diagrama cor-magnitude. Contudo se observa que há uma tendência maior para uma estrela mais massiva, embora um erro sistemático, proveniente da correção de avermelhamento, possa afetar os valores de $M$ obtidos. A rotação encontrada é próxima da média esperada para uma estrela Be. O valor de inclinação obtido, de $\cos i=0,01_{-0,01}^{+0,01}$, 


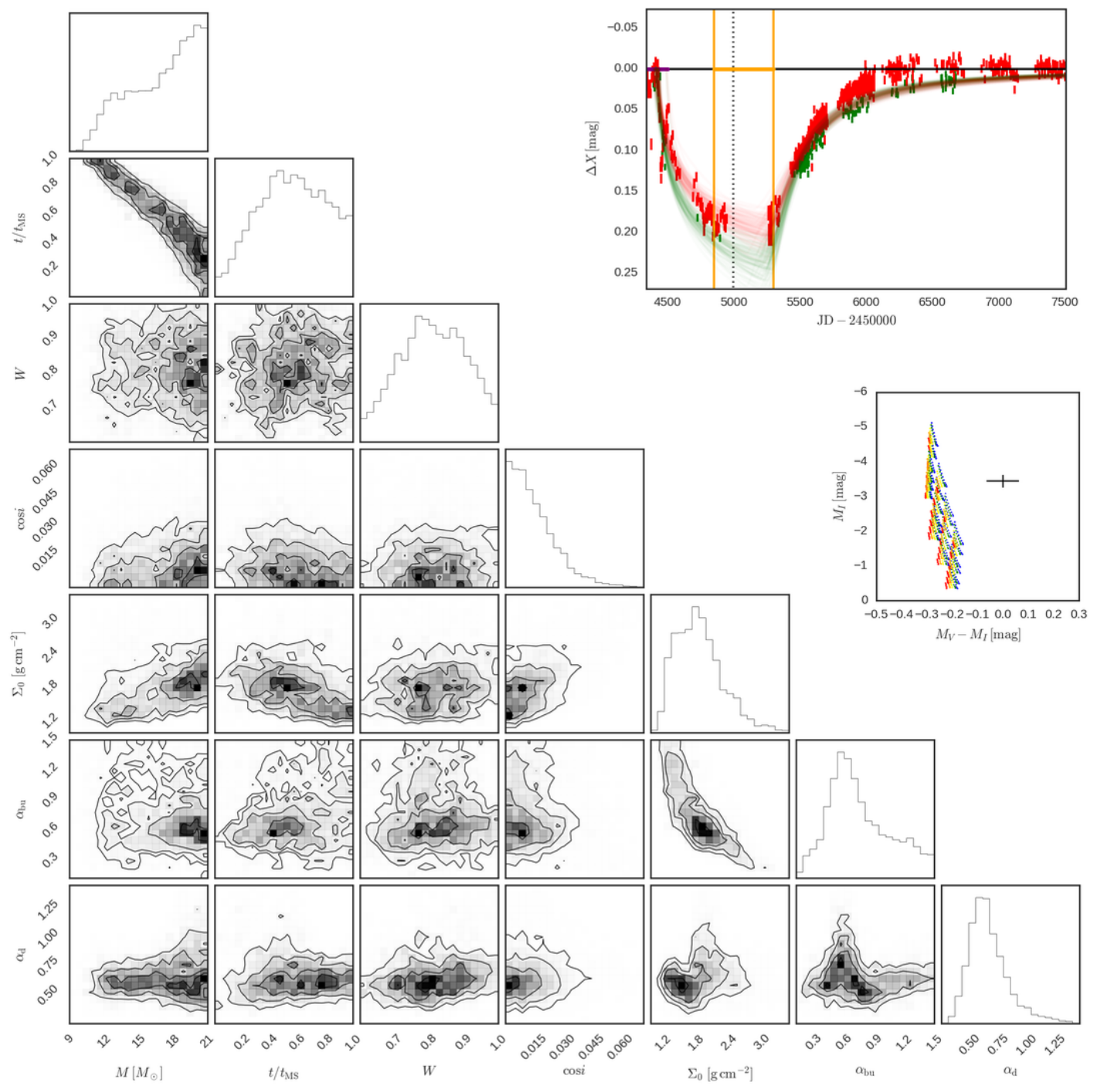

Figura 4.3: Direita superior: Curva de luz da estrela LMC 127.1.38725. Linhas finas: os 100 curvas de modelos aleatoriamente selecionadas a partir da amostra estacionária do código emcee. A cor vermelha (verde) indicam a banda fotométrica $I(V)$. Os intervalos de tempo roxo e laranja marcados em linhas horizontais são os intervalos permitidos para os parâmetros $t_{1}$ e $t_{2}$ do modelo, respectivamente. Direita intermediário: Diagrama cor-magnitude mostrando a grade de modelos de estrelas Be sem disco (tabela 2.3) e a posição de LMC 127.1.38725. Inferior: Resultados para a simulação do emcee para LMC 127.1.38725. O histograma das distribuições das probabilidades posteriores são mostrados nos painéis superiores e as correlações dois-a-dois dos parâmetros estelares $\left(M, W\right.$ e $\left.t / t_{\mathrm{ms}}\right)$, geométrico $(\cos i)$ e do bump $\left(\Sigma_{0}, \alpha_{\mathrm{bu}} \mathrm{e}\right.$ $\alpha_{\mathrm{d}}$ ) são mostrados nos painéis fora da diagonal. Os parâmetros $t_{1}$ e $t_{2}$ não são mostrados por conveniência. Os níveis de densidade normalizada mostrados nos painéis fora da diagonal são 12\%, 39\%, 68\%, 87\% do pico da probabilidade.

está em concordância com o esperado de uma estrela vista de lado. O erro inferido para a inclinação é baixo demais. Nossa experiência com o emcee mostra que isso pode ocorrer quando o espaço de parâmetros explorado é estreito demais. Novamente, acreditamos que isto seja devido a uma incorreta correção do avermelhamento, que coloca a estrela numa 


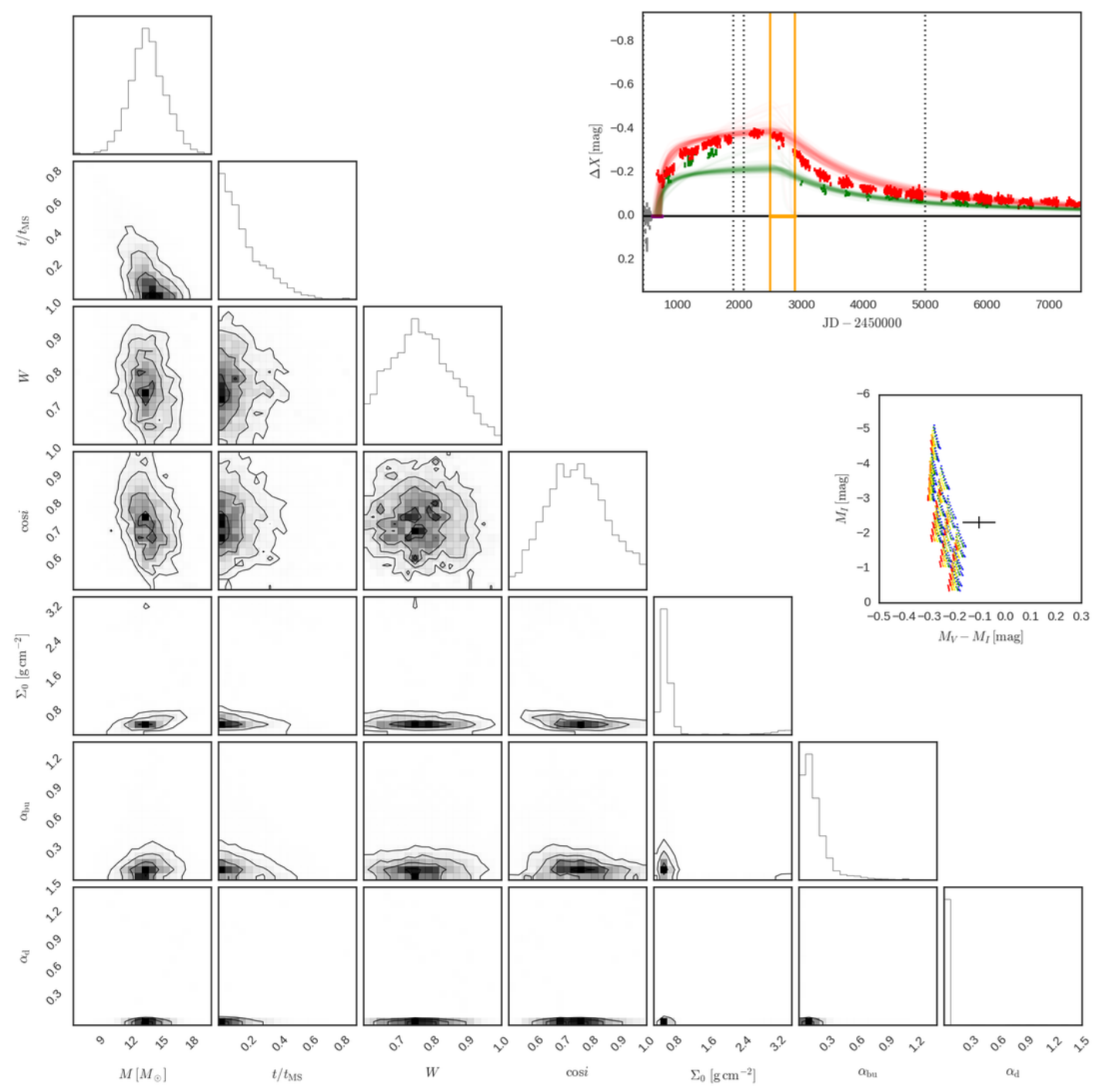

Figura 4.4: Idem à figura 2.8 para a estrela LMC 162.7.108765.

posição do diagrama cor-magnitude que não é coberta pela grade de modelos fotosféricos utilizada.

Os parâmetros do bump $\left(\Sigma_{0}, \alpha_{\mathrm{bu}}\right.$ e $\left.\alpha_{\mathrm{d}}\right)$ são restringidos pela forma geral do bump, onde a amplitude sofre maior influência de $\Sigma_{0}$ (e de $\cos i$ também), e os parâmetros de viscosidade para cada fase controlam a taxa de variação do brilho. A distribuição de $\Sigma_{0}$ de LMC 127.1.38725, é relativamente bem determinada por volta de $\sim 1,8 \mathrm{~g} \mathrm{~cm}^{-2}$, o que significa que se trata de um disco denso. Os parâmetros de viscosidade que melhor reproduzem os dados são $\alpha_{\mathrm{bu}}=0,69_{-0,22}^{+0,44}$ e $\alpha_{\mathrm{d}}=0,64_{-0,12}^{+0,18}$.

A fig. 4.4 é um exemplo de um VLB. Os resultados para LMC 162.7.108765 indicam uma estrela mais massiva $M=13,6_{-1,6}^{+1,8}$, cercada por um disco mais tênue $\Sigma_{0}=0,6_{-0,1}^{+0,1}$. 
A taxa de rotação obtida, que foi de $W \sim 0,77$, está próxima do valor médio esperado para uma estrela Be. O intervalo do ângulo de inclinação que corresponde a uma estrela vista do polo é bem maior do que o esperado para uma estrela vista de lado (como LMC 127.1.38725). Esse fato pode ser observado na distribuição mais alargada desse parâmetro, no quarto histograma da diagonal principal, como também nas incertezas obtidas para esse valor que foi de $\cos i=0,75_{-0,11}^{+0,12}$.

Nota-se, na fase de construção, que apesar do aumento inicial rápido em magnitude, o tempo entre o início do bump e a máxima amplitude observada é relativamente grande (da ordem de 2000 dias). Um resultado importante é que os modelos reproduzem bem essa característica observada. Ao se comparar a massa determinada pelo MCMC e a altura da estrela no diagrama cor magnitude, nota-se que há uma inconsistência. Provavelmente, a determinação da massa pelo MCMC foi prejudicada por um valor de avermelhamento excessivo, o que também deslocou a posição da estrela horizontalmente para a direita. Esse comportamento corresponde a pequenas taxas de massa injetada no disco e sua viscosidade, como confirmado pelos quinto e sexto histogramas da diagonal. A viscosidade durante a fase dissipativa também reflete esse comportamento. $\mathrm{O}$ valor desse parâmetro é de $\alpha_{\mathrm{d}}=0,01_{-0,01}^{+0,01}$, o que é uma característica marcante desse tipo de evento (VLB), cuja dissipação do disco é bastante demorada. Dentro de nosso conhecimento, este é o menor valor de $\alpha$ já registrado para uma estrela Be.

De forma geral, os histogramas da Fig. 4.3 são mais largos que os da Fig. 4.4, indicando que a determinação dos valores é pior para a estrela LMC 127.1.38725. Um fator determinante para que isso ocorra são as amplitudes menores observadas para dips, em comparação ao bumps, o que implica em medidas mais próximas ao nível de ruído das observações.

\subsection{Resultados para toda amostra de estrela}

Como visto anteriormente, a escassez de informações sobre a estrela central impacta a determinação dos parâmetros estelares. Dada a natureza do método MCMC, as incertezas desses parâmetros são propagadas para os parâmetros relativos ao bump. Portanto, a principal contribuição deste trabalho não reside na modelagem individual das estrelas que compõem a amostra aqui estudada, mas sim na obtenção de propriedades estatísticas da 


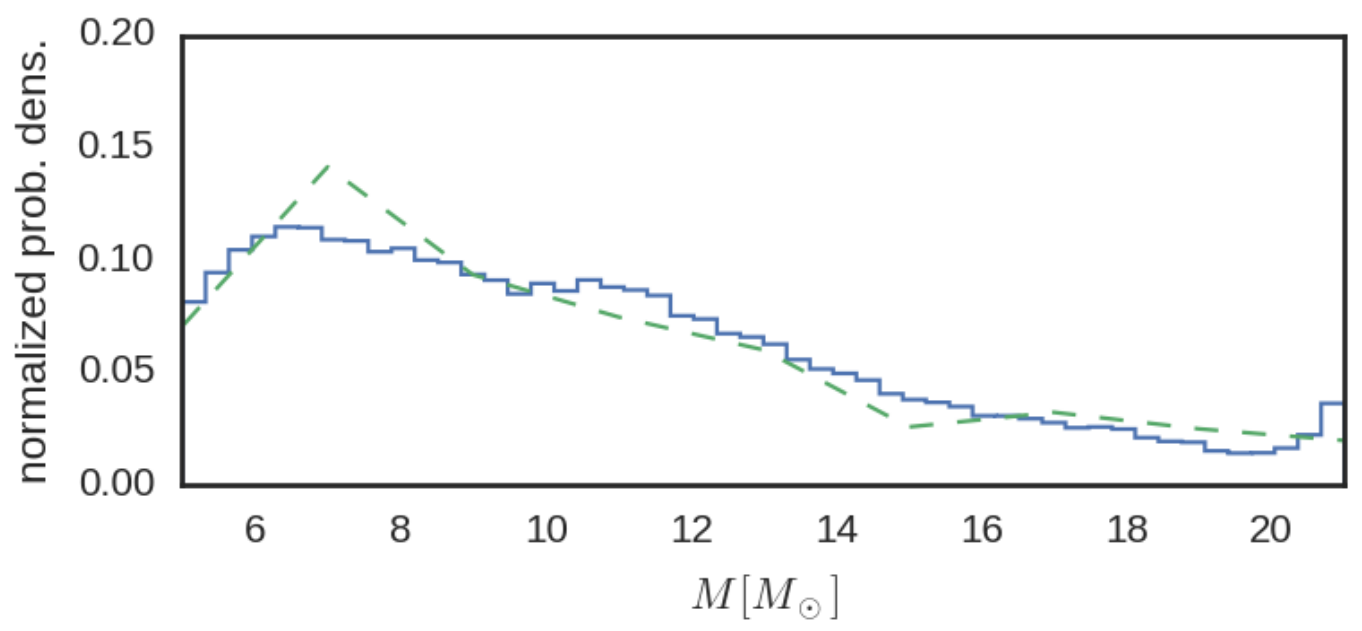

Figura 4.5: Histograma da soma das probabilidades posteriores do parâmetro $M$ para todas as estrelas modeladas (linha sólida). IMF de Kroupa (2001) ponderada pela razão entre estrela Be e B de Martayan et al. (2007), cujo fator é dado por $M^{2,3} f_{B e}(M)$ na Eq. 2.34 (linha sólida).

amostra como um todo. Nas próximas seções descrevemos as características obtida para a amostra, e sua comparação com a população de estrelas da SMC analisada por Rímulo (2017). Na tabela 4.2 estão listados os parâmetros obtidos para todos os eventos de disco analisados.

\subsubsection{Distribuição de Massa}

Como discutido no capítulo 1, o parâmetro principal que determina as características majoritárias da estrela é a massa. Para discutir as demais propriedades da amostra, precisamos determinar se as estrelas selecionadas são representativas de estrelas Be.

A somatória das probabilidades posteriores de $M$ é representada na Fig. 4.5 como uma linha contínua azul. Para comparação, a linha verde representa a fração entre Be e estrelas B, calculada por Martayan et al. (2007) a partir de um aglomerado da SMC. A mediana da massa para a amostra como um todo é de $\langle M\rangle=10.8_{-3,8}^{+5,8} M_{\odot}$.

Apesar dos possíveis efeitos devido ao avermelhamento, a distribuição da amostra é bem próxima à forma esperada para a fração de estrelas Be (Martayan et al., 2007). Rímulo et al. (2018) obtiveram uma distribuição com um pico de estrelas por volta de $\sim 11 M_{\odot}$. Um fator que pode alterar a fração de estrelas Be observadas é a distância. A LMC esta mais próxima de nós do que SMC, o que nos permite observar estrelas de menor brilho, ou seja, de menor massa. 

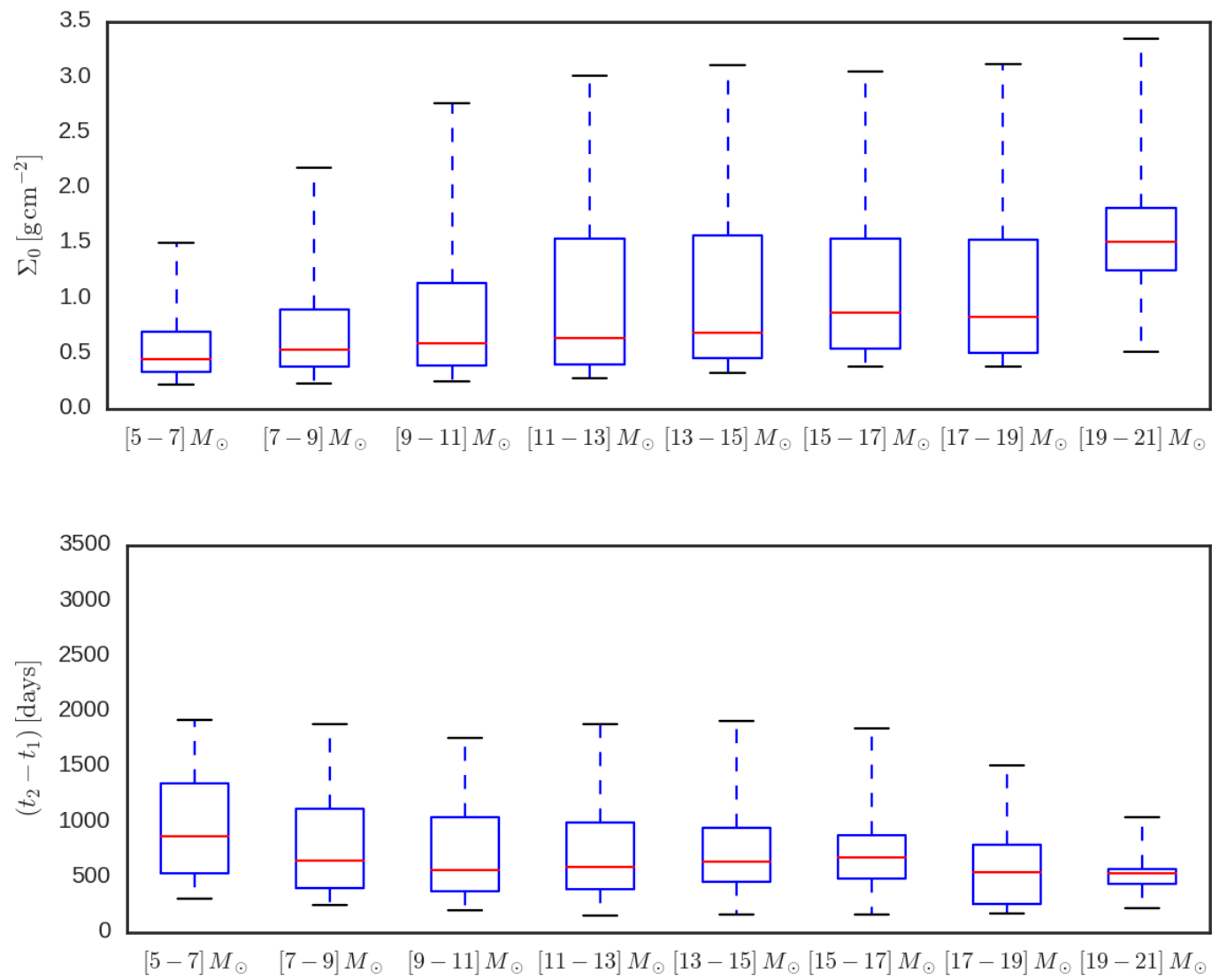

Figura 4.6: Gráfico de caixa para a soma das distribuições posteriores para $\Sigma_{0}$ (acima) e $t_{2}-t_{1}$ (abaixo) para a somatória das distribuições posteriores para os bumps da amostra, divididos em oito intervalos iguais de massa. As barras inferior e superior (em preto) marcam 5 e $95 \%$ da amostra, respectivamente. Os extremos da caixa (em azul) indicam 25 e $75 \%$ da amostra. A linha média (vermelha) aponta a mediana (50\%) da amostra.

\subsubsection{Densidade superficial assintótica}

A variação da densidade em função da massa estelar da nossa amostra é mostrada na parte superior da Fig 4.6. Há uma tendência de estrelas mais massivas possuírem densidades superficiais do disco maiores. Este resultado é compatível com o obtido por Vieira et al. (2017), onde os autores identificaram, para estrelas Be da Galáxia, que a densidade de base do disco cresce para estrelas mais massivas. O mesmo comportamento foi observado para a amostra de estrelas Be da SMC analisadas por Rímulo et al. (2018).

Na Fig. 4.7 mostramos a distribuição dos parâmetros $\Sigma_{0}$ e $M$. Para comparação, os resultados de Vieira et al. (2017) são representados por pontos vermelhos. Enquanto a amostra de Vieira et al. é melhor distribuída em massa e possui uma variedade mais ampla de $\Sigma_{0}$, a nossa amostra para a LMC é dominada por estrelas mais massivas e discos 


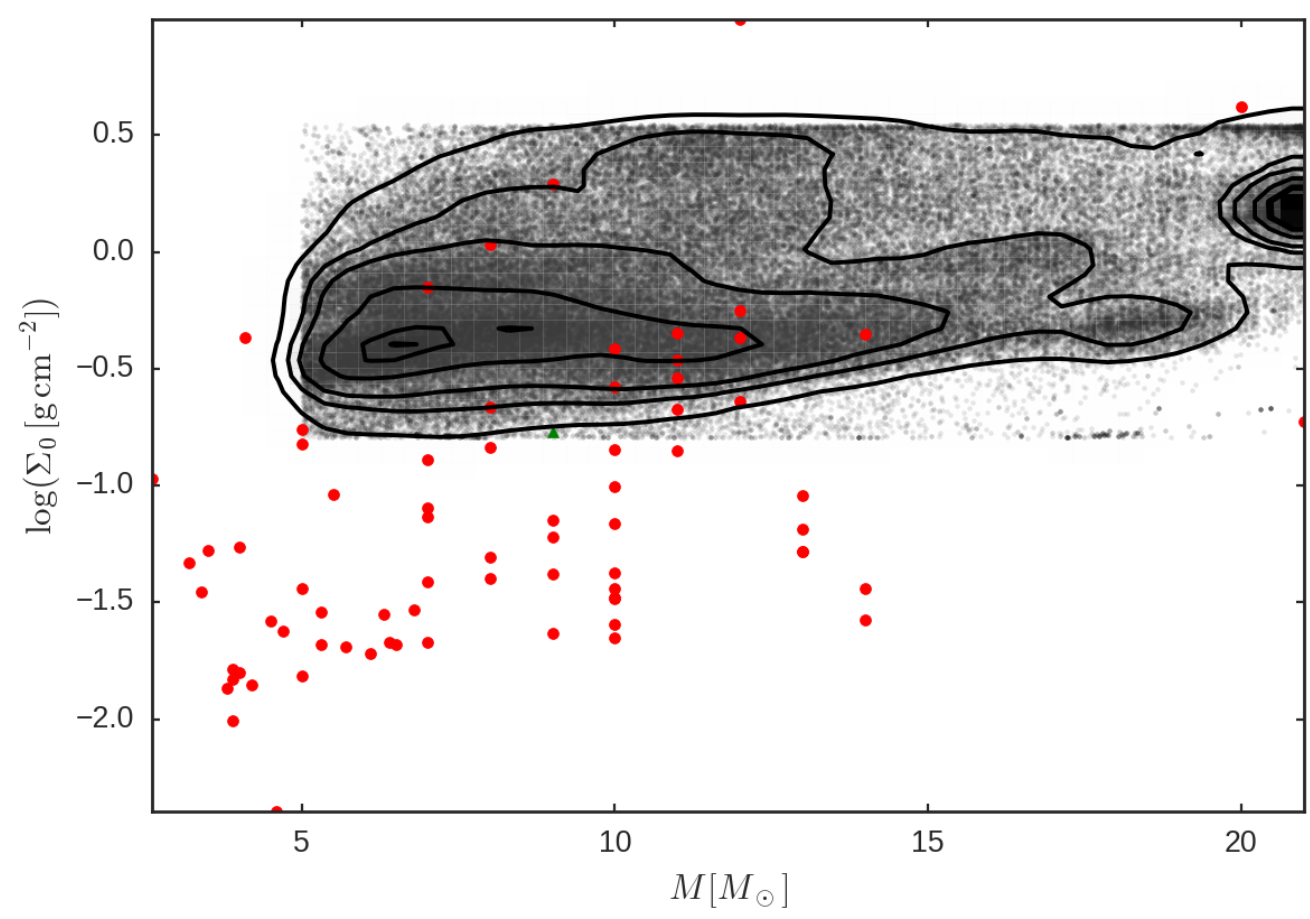

Figura 4.7: Distribuição dos parâmetros $M$ e $\Sigma_{0}$ da amostra de estrelas Be da LMC. As curvas de contorno são as mesmas utilizados na Fig. 4.3. Os pontos em vermelho representam as densidades superficiais na base do disco para estrelas Be da Via Láctea medidos por Vieira et al. (2017). O triângulo verde corresponde ao estado inicial de um modelos de disco ablativo de Kee et al. (2016) de uma estrela B2e.

mais densos. Essa diferença se deve, em parte, ao fato de Vieira et al. terem analisado a SED no infravermelho (entre 9 e $60 \mathrm{~cm}$ ), região em que a emissão dos discos é mais conspícua, o que os torna mais detectáveis. Por outro lado, a forma como selecionamos os eventos de disco (bumps bem definidos, excluindo os de pequena duração e amplitude), acaba por privilegiar eventos de maior densidade de disco. Além do mais, apenas discos mais massivos podem gerar excessos razoáveis no visível. Dessa forma, a nossa amostra deve representar um limite superior para $\Sigma_{0}$ das estrelas Be da LMC.

Os discos de nossa amostra são mais tênues, $\left\langle\Sigma_{0}\right\rangle=0,63_{-0,28}^{+1,09} \mathrm{~g} \mathrm{~cm}^{-2}$, se comparados com a amostra da SMC (Rímulo et al., 2018), cujo valor médio encontrado foi de $\left\langle\Sigma_{0}\right\rangle=$ $1,5_{-0,8}^{+1,1} \mathrm{~g} \mathrm{~cm}^{-2}$.

\subsubsection{Duração da fase de construção dos discos}

Na parte inferior da Fig. 4.6, apresentamos o tempo de duração da fase de construção do discos $\left(t_{2}-t_{1}\right)$, que demonstra uma tendência clara de que estrelas mais massivas têm 

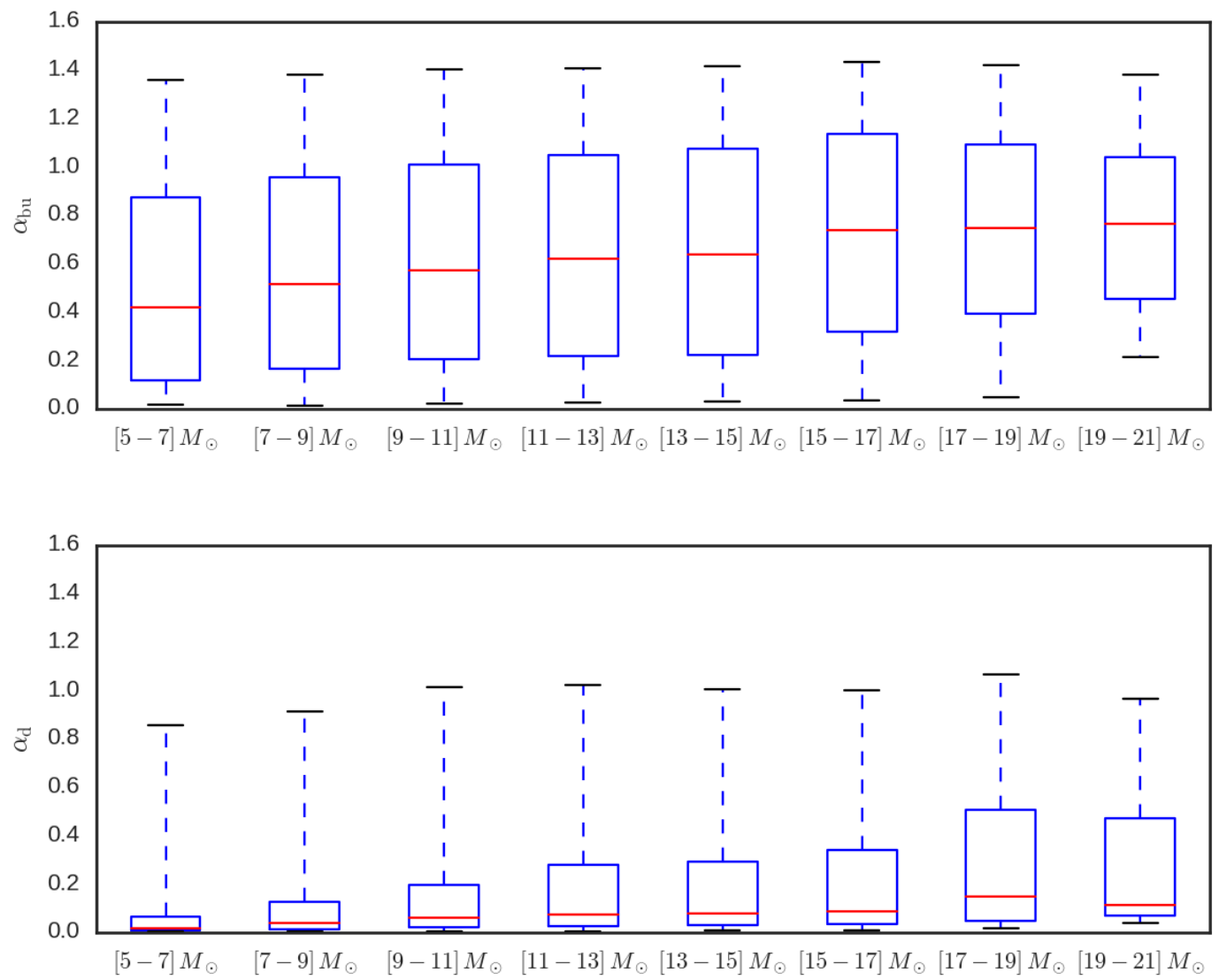

Figura 4.8: Idem à Fig. 4.6 para os parâmetros $\alpha_{\text {bu }}$ (acima) e $\alpha_{\mathrm{d}}$ (abaixo).

bumps de durações menores, o que indica que essas estrelas são mais variáveis que as mais tardias. Resultados semelhantes foram obtidos para a Via Láctea (Rivinius et al. 2013; Labadie-Bartz et al. 2017) e para a SMC (Rímulo et al., 2018).

Embora o mesmo comportamento de $t_{2}-t_{1}$ tenha sido observado para a SMC, os valores numéricos são bastante diferentes. Enquanto Rímulo et al. encontraram $\left\langle t_{2}-t_{1}\right\rangle=$ $3,04_{-1,68}^{+3,51} \times 10^{2}$ dias, obtivemos para nossa amostra $\left\langle t_{2}-t_{1}\right\rangle=6,35_{-3,04}^{+6,86} \times 10^{2}$ dias, o que pode ser um indício de que a metalicidade influencia a duração dos bumps. Claramente, nosso resultado é influenciado pela presença de vários VLBs em nossa amostra.

\subsubsection{Viscosidade}

Na Fig. 4.8 é apresentada a distribuição dos parâmetros $\alpha_{\mathrm{bu}}$ (superior) e $\alpha_{\mathrm{d}}$ (inferior) em função da massa da estrela. O parâmetro $\alpha_{\mathrm{bu}}$ cresce com a massa estelar e, aparentemente, $\alpha_{\mathrm{d}}$ se comporta da mesma forma. Rímulo et al. (2018) obtiveram que $\alpha_{\mathrm{bu}}$ não possui dependência com a massa e que há um leve indício de que $\alpha_{\mathrm{d}}$ é maior para estrelas 
Be recentes, analisando uma amostra da SMC. As medianas dos parâmetros de viscosidade possuem valores diferentes que correspondem a $\left\langle\alpha_{\mathrm{bu}}\right\rangle=0,59_{-0,49}^{+0,58}$ e $\left\langle\alpha_{\mathrm{d}}\right\rangle=0,06_{-0,05}^{+0,40}$, sendo dez vezes maior durante a fase construção do disco do que em sua dissipação. O resultado de $\alpha_{\mathrm{d}}$ é diferente do encontrado por Rímulo et al. $\left(2018,\left\langle\alpha_{\mathrm{d}}\right\rangle=0,26_{-0,18}^{+0,60}\right.$ e $\left.\left\langle\alpha_{\mathrm{bu}}\right\rangle=0,64_{-0,38}^{+0,50}\right)$, o que sugere que $\alpha_{\mathrm{d}}$ da LMC é dez vezes menor que na SMC. Essas estimativas do parâmetro $\alpha_{\mathrm{bu}}$, na ordem de alguns décimos, são compatíveis com os valores estimados para a estrela Be 28 CMa (Ghoreyshi et al., 2018) e com valores obtidos para discos de novas anãs (King et al. (2007); Kotko e Lasota (2012)). Especificamente, o valor obtido para $\alpha_{\mathrm{d}}$ para a LMC é compatível com a magnitude dos valores através de simulações hidrodinâmicas, onde a principal hipótese considerada para gerar viscosidade é a instabilidade magneto-rotacional (King et al., 2007).

Apesar dos trabalhos de Ghoreyshi et al. (2018) e Rímulo et al. (2018) obterem resultados semelhantes, ainda não está claro se $\alpha_{\mathrm{bu}}$ ser maior que $\alpha_{\mathrm{d}}$ é um fenômeno real ou resultado de aproximações utilizadas nos referidos trabalhos e neste estudo. Uma aproximação potencialmente problemática é a de disco isotérmico. Jones et al. (2004) e Carciofi et al. (2008) mostraram que essa aproximação não é realística. Consequentemente a expressão de $c_{\mathrm{s}}$ (Eq. 2.4) se torna uma função da distância à estrela, $R$. Rímulo et al. (2018) também argumenta que efeitos de forças radiativas também podem alterar os valores estimados de $\alpha$. Kee et al. (2016) simulou esse efeito, considerando discos não viscosos de metalicidade solar e opticamente finos. Os autores foram capazes de obter um disco que é destruído em escalas de tempo compatíveis com as variações fotométricas observadas para estrelas Be. Kee et al. argumentam que a atuação desse efeito pode gerar valores anormais de viscosidade, como os obtidos por Carciofi et al. (2012), Ghoreyshi et al. (2018), o que se estende também ao presente trabalho e ao de Rímulo et al. (2018). Contudo, espera-se que em ambientes de menor metalicidade esse efeito deve ser menor. Outro ponto importante é que os autores desse estudo utilizaram a aproximação de disco opticamente fino, o que não é válido para as regiões mais internas do disco. Outra explicação para a diferença de valores de $\alpha$ apontada por Rímulo et al. (2018) é o fato da injeção de massa ser um processo mecanicamente mais violento, com a injeção de matéria e energia de forma turbulenta. Por outro lado, o processo de dissipação deve ser mais suave, uma vez que provavelmente a atividade estelar não deve causar maiores perturbações nas condições do disco.

Em conclusão, o comportamento da viscosidade turbulenta em discos de estrelas Be está 
apenas começando a ser desvendado. Estudos do grupo de estrelas Be da USP indicam que $\alpha$ varia com o tempo, que seu valor é maior na construção do disco do que na dissipação. Os resultados desta dissertação indicam, pela primeira vez, uma possível dependência de $\alpha$ com a metalicidade.

\subsubsection{Taxas de perda de massa e momento angular}

Apesar dos discos de estrelas Be raramente se encontrarem em estado estacionário, as taxas de perda de massa e momento angular considerando esse regime são estimativas úteis para quantificar a perda real dessas quantidades pela estrela (veja Eqs. 2.17 e 2.18). Na Fig. 4.9 apresentamos a distribuição das taxas de perda de massa (acima) e momento angular (abaixo) para nossa amostra de estrelas Be, considerando discos em regime de estado estacionário. Os pontos vermelhos representam estimativas de $(-\partial M / \partial t)_{\text {est }}$ realizadas a partir dos resultados de Vieira et al. (2017) que analisou uma população de Be Galáticas, assumindo que $\alpha=1$. Na tabela 4.2 apresentamos os resultados da modelagem de todas as estrelas. Nas últimas três colunas estão os valores estimados das taxa de decréscimo típica, de perda de momento angular e o momento angular total perdido pela estrela durante o bump, $-\Delta J_{\star}$ (que é calculado pelo produto do tempo de duração da fase de construção do disco, $t_{2}-t_{1}$, vezes $\left.(-\partial J / \partial t)_{\mathrm{est}}\right)$.

As taxas de perda de massa e momento angular em estado estacionário para os discos mais densos de nossa amostra são da ordem de $\sim 5 \times 10^{-10} M_{\odot} \mathrm{ano}^{-1} \mathrm{e} \sim 10^{37} \mathrm{~g} \mathrm{~cm}^{2} \mathrm{~s}^{-1}$. Considerando o tempo médio de duração de $t_{2}-t_{1}$, que é da ordem de três anos $(\sim 1112$ dias), um bump denso completo deve resultar em uma perdas da ordem de $\sim 10^{-9} M_{\odot}$ e $\sim 10^{46} \mathrm{~g} \mathrm{~cm}^{2} \mathrm{~s}^{-1}$. Comparativamente, Rímulo et al. (2018) obtiveram perdas de $\sim 10^{-10} M_{\odot}$ $\mathrm{e} \sim 10^{44} \mathrm{~g} \mathrm{~cm}^{2} \mathrm{~s}^{-1}$, para as mesmas circunstâncias, considerando os bumps mais densos encontrados para a SMC (onde $t_{2}-t_{1}$ obtido de sua amostra é de $\sim 1$ ano). O surgimento de um disco é um mecanismo que pode remover quantidades significativas de momento angular, sem a necessidade de exista uma perda de massa grande.

Trabalhos recentes (Krtička et al., 2011) propuseram que o surgimento de um disco viscoso de decréscimo, ao longo da SP, é um mecanismo natural de extração de momento angular da estrela, o que a impediria de atingir a rotação crítica (note que na Fig. 2.6, a rotação estelar aumenta durante a evolução estelar). Granada et al. (2013), baseando-se nesse cenário, simularam o surgimento de um disco estacionário quando a rotação superfi- 
cial da estrela atinge $W>0,88$, estimando os valores de $(-\partial M / \partial t)_{\text {est }}$ e $(-\partial J / \partial t)_{\text {est }}$. Essas estimativas são mostradas na Fig. 4.9 pelas linhas azul (SMC) e verde (LMC). É notável que as estimativas dos parâmetros $(-\partial M / \partial t)_{\text {est }}$ e $(-\partial J / \partial t)_{\text {est }}$ obtidas por Granada et al. estão, aproximadamente, uma ordem de magnitude acima dos valores derivados por Vieira et al. (2017), para os discos mais densos da população da SMC (Rímulo et al., 2018) e do presente trabalho. Isso provavelmente significa que os modelos evolutivos de Genebra estão prevendo uma eficiência alta demais de transporte do momento angular ao longo da sequência principal. Estes resultados motivaram o início de uma colaboração entre o grupo de Genebra e o grupo da USP, visando entender as razões das discrepâncias reportadas acima. 

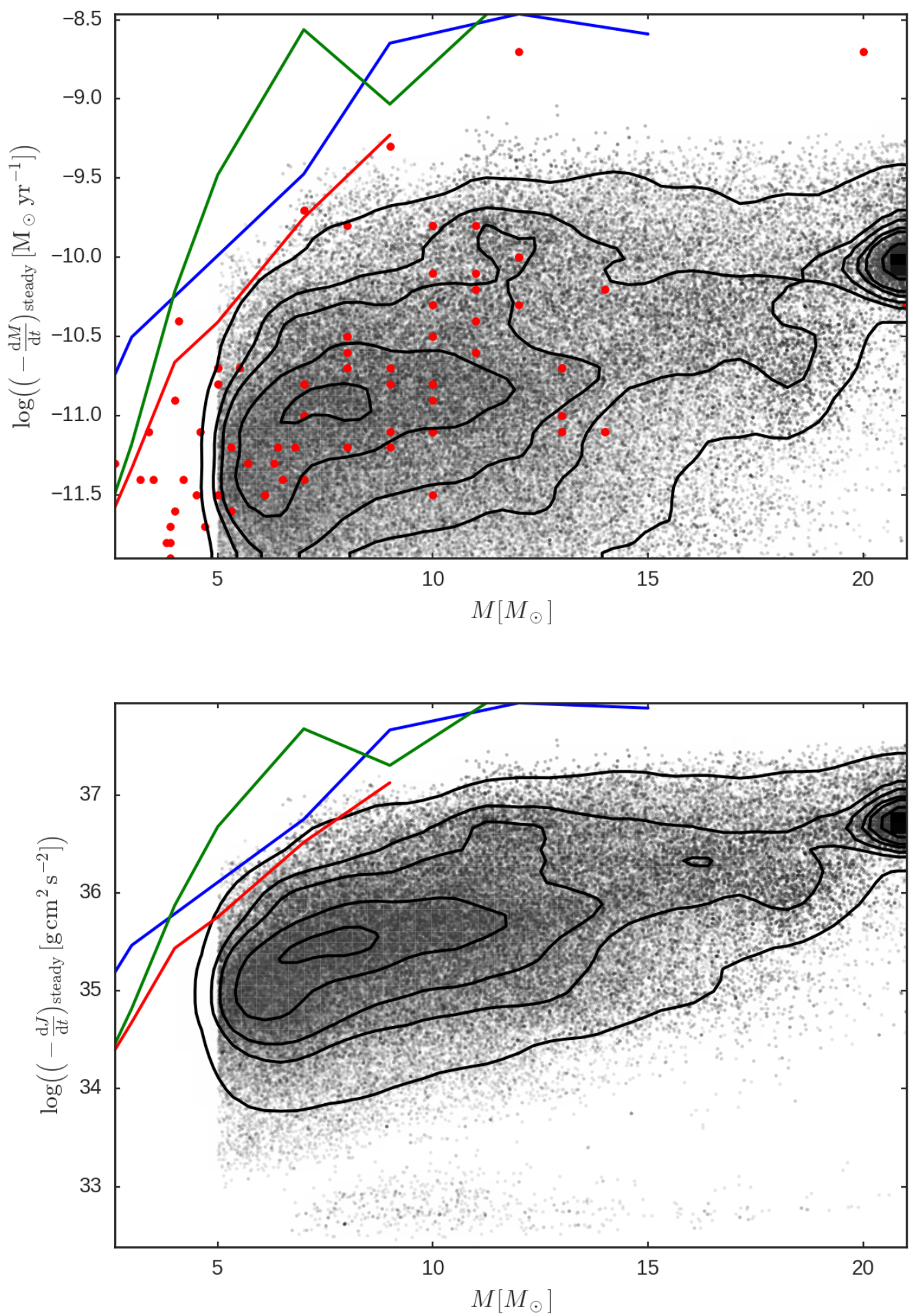

Figura 4.9: Distribuição das taxas de perda de massa e momento angular da amostra para o estado estacionário. As curvas de contorno utilizadas são as mesmas da Fig. 4.3. Os pontos vermelhos (imagem superior) são as estimativas de $(-\partial M / \partial t)_{\text {est }}$ calculadas a partir dos resultados de Vieira et al. (2017) para as Be Galáticas. As curvas em azul (verde) são as estimativas das taxas de perda de massa e momento angular para estrelas da SMC (LMC) durante episódios de formação de disco, considerando um disco em estado estacionário, feita por Granada et al. (2013). 
Tabela 4.2 - Resultados da pipeline para cada estrela e bump da amostra.

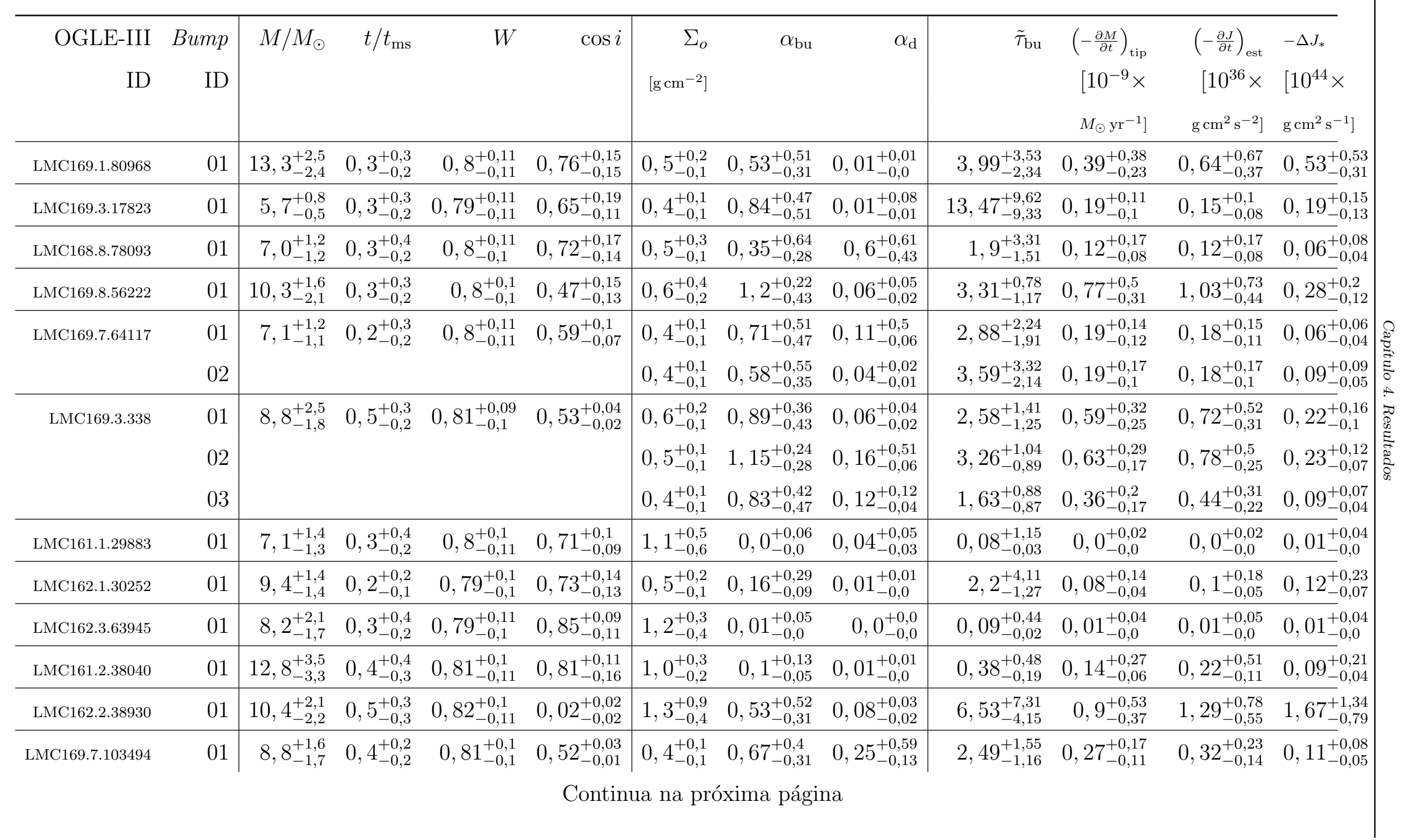


Tabela 4.2 - Continuação da página anterior

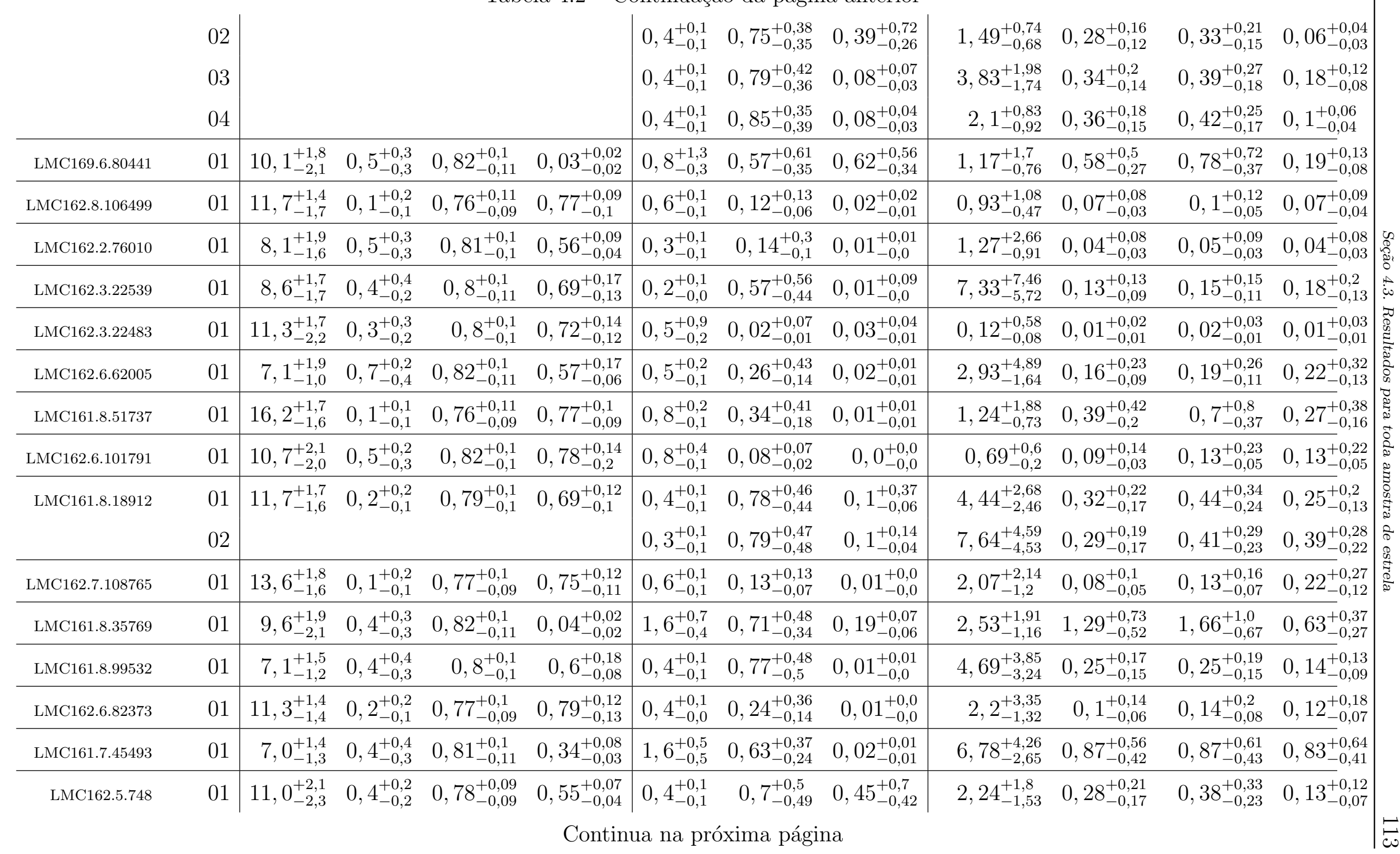


Tabela 4.2 - Continuação da página anterior

\begin{tabular}{|c|c|c|c|c|c|c|c|c|c|c|c|c|}
\hline & & & & & & & \\
\hline & 02 & & & & & $0,4_{-0,1}^{+0,1}$ & $0,7_{-0,45}^{+0,47}$ & $0,2_{-0,15}^{+0,87}$ & $1,95_{-1,26}^{+1,36}$ & $0,32_{-0,17}^{+0,24}$ & $0,44_{-0,23}^{+0,37}$ & $0,13_{-0,06}^{+0,11}$ \\
\hline & 03 & & & & & $0,4_{-0,1}^{+0,1}$ & $0,84_{-0,52}^{+0,4}$ & $0,42_{-0,31}^{+0,66}$ & $1,75_{-1,13}^{+0,97}$ & $0,36_{-0,19}^{+0,19}$ & $0,49_{-0,24}^{+0,31}$ & $0,11_{-0,05}^{+0,07}$ \\
\hline LMC161.8.1506 & 01 & $6,1_{-0,8}^{+1,0}$ & $0,4_{-0,3}^{+0,3}$ & $0,81_{-0,1}^{+0,1}$ & $0,64_{-0,1}^{+0,18}$ & $0,6_{-0,1}^{+0,2}$ & $0,58_{-0,39}^{+0,57}$ & $0,01_{-0,0}^{+0,0}$ & $6,39_{-4,53}^{+7,0}$ & $0,24_{-0,15}^{+0,25}$ & $0,22_{-0,13}^{+0,24}$ & $0,2_{-0,13}^{+0,25}$ \\
\hline \multirow[t]{2}{*}{ LMC161.8.84095 } & 01 & $11,5_{-2,2}^{+2,0}$ & $0,5_{-0,3}^{+0,3}$ & $0,82_{-0,1}^{+0,1}$ & $0,54_{-0,03}^{+0,04}$ & $0,7_{-0,1}^{+0,1}$ & $0,59_{-0,31}^{+0,53}$ & $0,09_{-0,02}^{+0,03}$ & $6,43_{-3,23}^{+5,52}$ & $0,54_{-0,27}^{+0,45}$ & $0,82_{-0,42}^{+0,71}$ & $\overline{0,97_{-0,49}^{+0,8}}$ \\
\hline & 02 & & & & & $0,9_{-0,2}^{+0,2}$ & $0,09_{-0,03}^{+0,06}$ & $0,01_{-0,0}^{+0,0}$ & $0,76_{-0,23}^{+0,49}$ & $0,12_{-0,04}^{+0,08}$ & $0,18_{-0,06}^{+0,14}$ & $0,16_{-0,06}^{+0,13}$ \\
\hline LMC100.2.89063 & 01 & $13,0_{-1,7}^{+1,8}$ & $0,1_{-0,1}^{+0,2}$ & $0,77_{-0,1}^{+0,11}$ & $0,76_{-0,11}^{+0,12}$ & $0,5_{-0,1}^{+0,1}$ & $0,24_{-0,17}^{+0,46}$ & $0,09_{-0,04}^{+0,08}$ & $1,22_{-0,83}^{+2,26}$ & $0,13_{-0,08}^{+0,23}$ & $0,19_{-0,12}^{+0,37}$ & $0,1_{-0,06}^{+0,19}$ \\
\hline \multirow[t]{3}{*}{ LMC103.3.78875 } & 01 & $16,3_{-1,5}^{+1,4}$ & $0,0_{-0,0}^{+0,1}$ & $0,71_{-0,07}^{+0,09}$ & $0,69_{-0,05}^{+0,04}$ & $1,0_{-0,1}^{+0,1}$ & $0,82_{-0,36}^{+0,42}$ & $0,05_{-0,01}^{+0,01}$ & $5,03_{-2,15}^{+2,51}$ & $1,02_{-0,43}^{+0,54}$ & $1,75_{-0,76}^{+1,02}$ & $1,09_{-0,47}^{+0,64}$ \\
\hline & 02 & & & & & $1,1_{-0,1}^{+0,1}$ & $0,74_{-0,22}^{+0,31}$ & $0,11_{-0,03}^{+0,03}$ & $3,76_{-1,14}^{+1,59}$ & $0,99_{-0,31}^{+0,5}$ & $1,72_{-0,59}^{+0,95}$ & $0,89_{-0,3}^{+0,51}$ \\
\hline & 03 & & & & & $0,9_{-0,1}^{+0,1}$ & $1,26_{-0,26}^{+0,16}$ & $0,32_{-0,06}^{+0,07}$ & $2,81_{-0,57}^{+0,38}$ & $1,36_{-0,32}^{+0,33}$ & $2,36_{-0,63}^{+0,74}$ & $0,54_{-0,15}^{+0,17}$ \\
\hline LMC103.4.102023 & 01 & $13,3_{-2,5}^{+3,3}$ & $0,7_{-0,3}^{+0,2}$ & $0,81_{-0,1}^{+0,1}$ & $0,55_{-0,04}^{+0,09}$ & $0,2_{-0,1}^{+0,2}$ & $0,33_{-0,29}^{+0,64}$ & $0,05_{-0,03}^{+0,61}$ & $1,44_{-1,27}^{+2,67}$ & $0,14_{-0,12}^{+0,25}$ & $0,27_{-0,22}^{+0,48}$ & $0,15_{-0,13}^{+0,27}$ \\
\hline LMC100.1.40815 & 01 & $7,5_{-1,3}^{+1,4}$ & $0,3_{-0,2}^{+0,3}$ & $0,8_{-0,11}^{+0,11}$ & $0,67_{-0,12}^{+0,17}$ & $0,4_{-0,1}^{+1,2}$ & $0,44_{-0,41}^{+0,69}$ & $0,05_{-0,03}^{+0,07}$ & $0,93_{-0,87}^{+1,72}$ & $0,12_{-0,08}^{+0,14}$ & $0,12_{-0,08}^{+0,15}$ & $0,02_{-0,01}^{+0,04}$ \\
\hline LMC100.1.116840 & 01 & $8,6_{-1,2}^{+1,3}$ & $0,2_{-0,1}^{+0,2}$ & $0,79_{-0,1}^{+0,1}$ & $0,72_{-0,13}^{+0,14}$ & $0,6_{-0,1}^{+0,2}$ & $0,15_{-0,08}^{+0,51}$ & $0,01_{-0,0}^{+0,01}$ & $0,79_{-0,43}^{+2,79}$ & $0,08_{-0,04}^{+0,22}$ & $0,09_{-0,05}^{+0,27}$ & $0,04_{-0,02}^{+0,13}$ \\
\hline LMC100.1.133871 & 01 & $17,0_{-2,2}^{+2,6}$ & $0,9_{-0,1}^{+0,1}$ & $0,81_{-0,1}^{+0,1}$ & $0,55_{-0,04}^{+0,09}$ & $0,6_{-0,2}^{+0,2}$ & $0,17_{-0,1}^{+0,39}$ & $0,02_{-0,01}^{+0,01}$ & $0,9_{-0,53}^{+2,07}$ & $0,37_{-0,21}^{+0,63}$ & $0,99_{-0,59}^{+1,66}$ & $0,9_{-0,52}^{+1,55}$ \\
\hline \multirow[t]{4}{*}{ LMC100.1.95208 } & 01 & $18,5_{-0,9}^{+1,0}$ & $0,1_{-0,1}^{+0,1}$ & $0,79_{-0,09}^{+0,09}$ & $0,61_{-0,04}^{+0,03}$ & $0,5_{-0,1}^{+0,1}$ & $0,66_{-0,35}^{+0,42}$ & $0,04_{-0,01}^{+0,02}$ & $1,2_{-0,63}^{+0,74}$ & $0,52_{-0,23}^{+0,31}$ & $1,06_{-0,47}^{+0,65}$ & $\overline{0,23_{-0,1}^{+0,13}}$ \\
\hline & 02 & & & & & $0,5_{-0,1}^{+0,1}$ & $0,64_{-0,31}^{+0,39}$ & $0,31_{-0,15}^{+0,33}$ & $2,77_{-1,36}^{+1,8}$ & $0,49_{-0,22}^{+0,31}$ & $0,99_{-0,46}^{+0,65}$ & $0,51_{-0,24}^{+0,34}$ \\
\hline & 03 & & & & & $0,4_{-0,0}^{+0,1}$ & $0,86_{-0,34}^{+0,35}$ & $0,27_{-0,16}^{+0,38}$ & $3,97_{-1,58}^{+1,63}$ & $0,54_{-0,22}^{+0,23}$ & $1,09_{-0,45}^{+0,5}$ & $0,59_{-0,25}^{+0,28}$ \\
\hline & 04 & & & & & $0,5_{-0,1}^{+0,1}$ & $0,69_{-0,35}^{+0,4}$ & $0,08_{-0,02}^{+0,03}$ & $2,37_{-1,13}^{+1,31}$ & $0,58_{-0,29}^{+0,32}$ & $1,19_{-0,59}^{+0,67}$ & $0,47_{-0,23}^{+0,26}$ \\
\hline \multirow[t]{2}{*}{ LMC100.8.129218 } & 01 & $18,7_{-3,3}^{+1,7}$ & $0,5_{-0,2}^{+0,2}$ & $0,78_{-0,09}^{+0,1}$ & $0,0_{-0,0}^{+0,01}$ & $0,9_{-0,1}^{+0,2}$ & $1,0_{-0,38}^{+0,34}$ & $0,87_{-0,3}^{+0,39}$ & $5,56_{-2,14}^{+1,92}$ & $1,89_{-0,57}^{+0,72}$ & $4,18_{-1,43}^{+1,93}$ & $3,18_{-1,03}^{+1,36}$ \\
\hline & 02 & & & & & $1,5_{-0,2}^{+0,3}$ & $1,16_{-0,34}^{+0,24}$ & $0,48_{-0,12}^{+0,2}$ & $2,05_{-0,65}^{+0,59}$ & $3,56_{-0,88}^{+0,98}$ & $8,02_{-2,3}^{+2,59}$ & $1,94_{-0,59}^{+0,71}$ \\
\hline
\end{tabular}


Tabela 4.2 - Continuação da página anterior

\begin{tabular}{|c|c|c|c|c|c|c|c|c|c|c|c|c|}
\hline & 03 & & & & & $1,7_{-0,4}^{+0,5}$ & $0,82_{-0,3}^{+0,31}$ & $0,52_{-0,17}^{+0,34}$ & $1,64_{-0,62}^{+1,07}$ & $2,84_{-0,8}^{+0,93}$ & $6,35_{-2,0}^{+2,46}$ & $1,77_{-0,63}^{+0,96}$ \\
\hline LMC100.6.50481 & 01 & $13,7_{-2,6}^{+3,9}$ & $0,7_{-0,3}^{+0,2}$ & $0,82_{-0,11}^{+0,1}$ & $0,56_{-0,05}^{+0,14}$ & $0,4_{-0,1}^{+1,0}$ & $0,44_{-0,39}^{+0,68}$ & $0,09_{-0,04}^{+0,16}$ & $0,32_{-0,29}^{+0,51}$ & $0,35_{-0,21}^{+0,44}$ & $0,69_{-0,41}^{+0,87}$ & $0,06_{-0,04}^{+0,09}$ \\
\hline LMC100.6.34598 & 01 & $10,2_{-2,0}^{+1,5}$ & $0,3_{-0,2}^{+0,3}$ & $0,8_{-0,1}^{+0,1}$ & $0,71_{-0,13}^{+0,15}$ & $0,5_{-0,1}^{+0,2}$ & $0,71_{-0,45}^{+0,46}$ & $0,03_{-0,01}^{+0,02}$ & $2,12_{-1,41}^{+1,54}$ & $0,33_{-0,18}^{+0,26}$ & $0,43_{-0,23}^{+0,37}$ & $0,13_{-0,07}^{+0,12}$ \\
\hline LMC100.1.1702 & 01 & $6,5_{-0,9}^{+1,2}$ & $0,3_{-0,2}^{+0,3}$ & $0,8_{-0,11}^{+0,1}$ & $0,61_{-0,08}^{+0,18}$ & $0,5_{-0,1}^{+0,1}$ & $0,56_{-0,4}^{+0,58}$ & $0,01_{-0,0}^{+0,01}$ & $4,79_{-3,35}^{+5,08}$ & $0,19_{-0,13}^{+0,21}$ & $0,18_{-0,12}^{+0,2}$ & $0,13_{-0,08}^{+0,16}$ \\
\hline LMC100.8.18112 & 01 & $7,9_{-1,6}^{+1,7}$ & $0,4_{-0,3}^{+0,4}$ & $0,81_{-0,1}^{+0,1}$ & $0,39_{-0,07}^{+0,16}$ & $0,4_{-0,1}^{+0,2}$ & $0,58_{-0,4}^{+0,56}$ & $0,01_{-0,01}^{+0,01}$ & $9,0_{-6,31}^{+9,51}$ & $0,22_{-0,14}^{+0,23}$ & $0,24_{-0,15}^{+0,27}$ & $0,35_{-0,23}^{+0,41}$ \\
\hline \multirow[t]{2}{*}{ LMC100.8.91619 } & 01 & $15,7_{-2,0}^{+1,7}$ & $0,1_{-0,1}^{+0,2}$ & $0,74_{-0,09}^{+0,09}$ & $0,75_{-0,11}^{+0,09}$ & $0,4_{-0,1}^{+0,1}$ & $0,77_{-0,46}^{+0,46}$ & $0,01_{-0,0}^{+0,28}$ & $6,47_{-4,11}^{+4,78}$ & $0,39_{-0,23}^{+0,27}$ & $0,67_{-0,39}^{+0,52}$ & $0,6_{-0,38}^{+0,54}$ \\
\hline & 02 & & & & & $0,4_{-0,1}^{+0,1}$ & $1,03_{-0,47}^{+0,32}$ & $0,06_{-0,02}^{+0,25}$ & $8,14_{-3,71}^{+2,76}$ & $0,48_{-0,22}^{+0,2}$ & $0,81_{-0,38}^{+0,43}$ & $0,67_{-0,32}^{+0,33}$ \\
\hline LMC100.8.91630 & 01 & $11,4_{-2,6}^{+2,4}$ & $0,5_{-0,3}^{+0,3}$ & $0,82_{-0,1}^{+0,1}$ & $0,58_{-0,06}^{+0,14}$ & $0,5_{-0,1}^{+0,2}$ & $0,11_{-0,06}^{+0,14}$ & $0,01_{-0,0}^{+0,0}$ & $1,89_{-1,16}^{+2,66}$ & $0,08_{-0,05}^{+0,1}$ & $0,12_{-0,07}^{+0,17}$ & $0,24_{-0,14}^{+0,34}$ \\
\hline \multirow[t]{2}{*}{ LMC103.5.76737 } & 01 & $13,8_{-2,8}^{+3,4}$ & $0,6_{-0,4}^{+0,3}$ & $0,81_{-0,11}^{+0,11}$ & $0,01_{-0,01}^{+0,02}$ & $1,6_{-0,3}^{+0,6}$ & $0,9_{-0,44}^{+0,41}$ & $0,28_{-0,06}^{+0,06}$ & $1,47_{-0,68}^{+0,72}$ & $2,52_{-0,87}^{+0,95}$ & $4,67_{-1,72}^{+2,05}$ & $0,96_{-0,32}^{+0,42}$ \\
\hline & 02 & & & & & $2,7_{-0,5}^{+0,5}$ & $0,94_{-0,38}^{+0,39}$ & $0,41_{-0,11}^{+0,15}$ & $0,99_{-0,44}^{+0,6}$ & $4,29_{-1,38}^{+1,55}$ & $8,01_{-2,76}^{+3,23}$ & $1,08_{-0,41}^{+0,59}$ \\
\hline LMC100.8.19248 & 01 & $6,0_{-0,7}^{+0,9}$ & $0,3_{-0,2}^{+0,3}$ & $0,8_{-0,11}^{+0,1}$ & $0,59_{-0,07}^{+0,15}$ & $0,2_{-0,0}^{+0,1}$ & $0,43_{-0,37}^{+0,64}$ & $0,33_{-0,33}^{+0,76}$ & $2,58_{-2,15}^{+4,8}$ & $0,06_{-0,05}^{+0,08}$ & $0,05_{-0,04}^{+0,07}$ & $0,02_{-0,02}^{+0,04}$ \\
\hline LMC100.7.34608 & 01 & $10,8_{-2,8}^{+2,3}$ & $0,4_{-0,3}^{+0,4}$ & $0,81_{-0,1}^{+0,1}$ & $0,77_{-0,13}^{+0,13}$ & $1,1_{-0,3}^{+0,5}$ & $0,02_{-0,01}^{+0,05}$ & $0,01_{-0,0}^{+0,0}$ & $0,12_{-0,05}^{+0,34}$ & $0,03_{-0,01}^{+0,04}$ & $0,04_{-0,02}^{+0,07}$ & $0,03_{-0,01}^{+0,05}$ \\
\hline LMC100.7.18811 & 01 & $5,6_{-0,4}^{+0,6}$ & $0,2_{-0,1}^{+0,2}$ & $0,79_{-0,1}^{+0,11}$ & $0,59_{-0,06}^{+0,13}$ & $0,3_{-0,1}^{+0,1}$ & $0,55_{-0,43}^{+0,61}$ & $0,0_{-0,0}^{+0,0}$ & $10,63_{-8,28}^{+11,23}$ & $0,11_{-0,08}^{+0,11}$ & $0,08_{-0,06}^{+0,09}$ & $0,12_{-0,09}^{+0,13}$ \\
\hline LMC111.2.37142 & 01 & $8,6_{-1,9}^{+1,7}$ & $0,4_{-0,3}^{+0,4}$ & $0,81_{-0,1}^{+0,11}$ & $0,36_{-0,04}^{+0,11}$ & $0,8_{-0,3}^{+0,6}$ & $0,27_{-0,19}^{+0,46}$ & $0,04_{-0,02}^{+0,03}$ & $1,71_{-1,15}^{+2,66}$ & $0,21_{-0,12}^{+0,28}$ & $0,25_{-0,15}^{+0,34}$ & $0,15_{-0,09}^{+0,2}$ \\
\hline LMC111.2.87298 & 01 & $11,3_{-2,1}^{+1,7}$ & $0,3_{-0,2}^{+0,3}$ & $0,8_{-0,09}^{+0,1}$ & $\begin{array}{l}0,59_{-0,06}^{+0,09} \\
\end{array}$ & $0,3_{-0,1}^{+0,2}$ & $0,6_{-0,51}^{+0,56}$ & $0,06_{-0,04}^{+0,19}$ & $0,72_{-0,61}^{+0,75}$ & $0,17_{-0,11}^{+0,14}$ & $0,24_{-0,16}^{+0,21}$ & $\begin{array}{r}0,03_{-0,02}^{+0,03} \\
\end{array}$ \\
\hline
\end{tabular}

Continua na próxima página 
Tabela 4.2 - Continuação da página anterior

\begin{tabular}{|c|c|c|c|c|c|c|c|c|c|c|c|c|}
\hline & 02 & & & & & $0,6_{-0,1}^{+0,3}$ & $1,2_{-0,41}^{+0,22}$ & $0,08_{-0,03}^{+0,06}$ & $0,96_{-0,5}^{+0,51}$ & $0,88_{-0,24}^{+0,27}$ & $1,24_{-0,38}^{+0,48}$ & $0,1_{-0,04}^{+0,05}$ \\
\hline LMC111.3.61518 & 01 & $6,1_{-0,7}^{+0,9}$ & $0,4_{-0,2}^{+0,3}$ & $0,8_{-0,1}^{+0,1}$ & $0,57_{-0,05}^{+0,11}$ & $0,3_{-0,1}^{+0,1}$ & $0,34_{-0,28}^{+0,53}$ & $0,0_{-0,0}^{+0,36}$ & $2,36_{-1,9}^{+3,58}$ & $0,07_{-0,05}^{+0,1}$ & $0,06_{-0,04}^{+0,09}$ & $\begin{array}{c}0,04_{-0,03}^{+0,05} \\
\end{array}$ \\
\hline LMC111.2.74618 & 02 & $8,0_{-1,4}^{+1,5}$ & $0,3_{-0,2}^{+0,3}$ & $0,79_{-0,1}^{+0,1}$ & $0,75_{-0,2}^{+0,17}$ & $0,4_{-0,1}^{+0,6}$ & $0,98_{-0,72}^{+0,37}$ & $0,11_{-0,04}^{+0,08}$ & $2,42_{-1,82}^{+0,92}$ & $0,34_{-0,15}^{+0,17}$ & $0,36_{-0,15}^{+0,22}$ & $0,08_{-0,04}^{+0,05}$ \\
\hline LMC111.3.23404 & 01 & $11,4_{-1,5}^{+1,5}$ & $0,2_{-0,1}^{+0,2}$ & $0,79_{-0,1}^{+0,11}$ & $0,75_{-0,11}^{+0,13}$ & $0,4_{-0,1}^{+0,1}$ & $0,29_{-0,22}^{+0,56}$ & $0,02_{-0,01}^{+0,01}$ & $3,82_{-2,82}^{+6,88}$ & $0,13_{-0,09}^{+0,25}$ & $0,18_{-0,12}^{+0,35}$ & $0,22_{-0,15}^{+0,41}$ \\
\hline \multirow[t]{2}{*}{ LMC111.7.70752 } & 01 & $11,9_{-2,5}^{+2,1}$ & $0,5_{-0,3}^{+0,3}$ & $0,82_{-0,12}^{+0,1}$ & $0,05_{-0,03}^{+0,02}$ & $2,1_{-0,7}^{+0,7}$ & $0,86_{-0,43}^{+0,44}$ & $0,47_{-0,2}^{+0,31}$ & $1,92_{-1,02}^{+1,32}$ & $2,28_{-1,11}^{+1,6}$ & $\begin{array}{l}3,55_{-1,77}^{+2,66} \\
\end{array}$ & $\overline{0,9_{-0,45}^{+0}, 71}$ \\
\hline & 02 & & & & & $1,8_{-0,6}^{+0,7}$ & $0,84_{-0,43}^{+0,45}$ & $0,66_{-0,25}^{+0,37}$ & $3,35_{-1,75}^{+1,89}$ & $1,86_{-0,8}^{+1,31}$ & $2,9_{-1,29}^{+2,09}$ & $1,29_{-0,59}^{+0,92}$ \\
\hline LMC111.3.50562 & 01 & $6,7_{-0,6}^{+0,8}$ & $0,9_{-0,1}^{+0,1}$ & $0,85_{-0,1}^{+0,08}$ & $0,51_{-0,01}^{+0,02}$ & $0,6_{-0,1}^{+0,1}$ & $0,05_{-0,02}^{+0,02}$ & $0,01_{-0,0}^{+0,0}$ & $0,7_{-0,22}^{+0,29}$ & $0,04_{-0,01}^{+0,02}$ & $0,05_{-0,02}^{+0,02}$ & $0,08_{-0,02}^{+0,04}$ \\
\hline LMC110.8.74753 & 01 & $15,3_{-2,3}^{+3,9}$ & $0,8_{-0,2}^{+0,1}$ & $0,81_{-0,1}^{+0,1}$ & $0,54_{-0,03}^{+0,06}$ & $1,6_{-0,3}^{+0,6}$ & $0,91_{-0,28}^{+0,44}$ & $0,22_{-0,05}^{+0,05}$ & $7,97_{-2,32}^{+3,58}$ & $\begin{array}{r}4,55_{-1,79}^{+2,02} \\
\end{array}$ & $10,37_{-4,33}^{+5,55}$ & $13,76_{-5,5}^{+7,1}$ \\
\hline \multirow[t]{2}{*}{ LMC111.6.31665 } & 01 & $10,6_{-2,4}^{+1,8}$ & $0,5_{-0,3}^{+0,3}$ & $0,82_{-0,11}^{+0,1}$ & $0,04_{-0,02}^{+0,02}$ & $2,7_{-0,6}^{+0,5}$ & $0,92_{-0,49}^{+0,38}$ & $0,13_{-0,05}^{+0,08}$ & $2,93_{-1,64}^{+2,2}$ & $2,88_{-1,38}^{+1,67}$ & $4,08_{-2,0}^{+2,48}$ & $1,41_{-0,75}^{+1,24}$ \\
\hline & 02 & & & & & $1,7_{-0,6}^{+0,6}$ & $0,85_{-0,45}^{+0,42}$ & $0,28_{-0,13}^{+0,23}$ & $1,87_{-1,0}^{+1,1}$ & $1,58_{-0,68}^{+1,15}$ & $2,26_{-0,99}^{+1,58}$ & $0,54_{-0,24}^{+0,39}$ \\
\hline LMC110.8.65012 & 01 & $10,9_{-2,2}^{+2,6}$ & $0,6_{-0,3}^{+0,3}$ & $0,81_{-0,1}^{+0,1}$ & $0,03_{-0,02}^{+0,02}$ & $2,8_{-0,5}^{+0,5}$ & $0,94_{-0,38}^{+0,38}$ & $0,67_{-0,22}^{+0,3}$ & $2,68_{-1,1}^{+1,32}$ & $3,59_{-1,31}^{+1,57}$ & $5,46_{-2,07}^{+2,71}$ & $1,74_{-0,67}^{+0,94}$ \\
\hline \multirow[t]{2}{*}{ LMC110.7.60841 } & 01 & $10,9_{-2,4}^{+2,1}$ & $0,4_{-0,3}^{+0,3}$ & $0,83_{-0,1}^{+0,1}$ & $0,02_{-0,01}^{+0,01}$ & $1,4_{-0,3}^{+0,7}$ & $0,75_{-0,43}^{+0,5}$ & $0,35_{-0,16}^{+0,61}$ & $2,07_{-1,18}^{+1,69}$ & $1,26_{-0,52}^{+0,65}$ & $1,8_{-0,75}^{+1,06}$ & $0,53_{-0,22}^{+0,31}$ \\
\hline & 02 & & & & & $1,1_{-0,2}^{+0,5}$ & $0,67_{-0,4}^{+0,48}$ & $0,16_{-0,08}^{+0,09}$ & $3,57_{-2,26}^{+2,69}$ & $0,94_{-0,4}^{+0,5}$ & $1,36_{-0,6}^{+0,75}$ & $0,76_{-0,36}^{+0,48}$ \\
\hline LMC111.7.50820 & 01 & $10,6_{-2,8}^{+6,7}$ & $0,7_{-0,4}^{+0,2}$ & $0,85_{-0,09}^{+0,08}$ & $0,07_{-0,02}^{+0,03}$ & $0,2_{-0,0}^{+0,1}$ & $0,0_{-0,0}^{+0,57}$ & $0,87_{-0,43}^{+0,38}$ & $0,02_{-0,01}^{+7,91}$ & $0,0_{-0,0}^{+0,15}$ & $0,0_{-0,0}^{+0,28}$ & $0,0_{-0,0}^{+0,49}$ \\
\hline LMC111.5.53741 & 01 & $9,9_{-1,7}^{+1,5}$ & $0,2_{-0,2}^{+0,3}$ & $0,8_{-0,1}^{+0,1}$ & $0,71_{-0,12}^{+0,14}$ & $0,4_{-0,1}^{+0,1}$ & $0,25_{-0,18}^{+0,56}$ & $0,03_{-0,01}^{+0,03}$ & $3,68_{-2,7}^{+7,98}$ & $0,09_{-0,06}^{+0,2}$ & $0,11_{-0,08}^{+0,25}$ & $0,16_{-0,11}^{+0,34}$ \\
\hline \multirow[t]{2}{*}{ LMC119.4.80248 } & 01 & $16,3_{-2,9}^{+2,5}$ & $0,4_{-0,2}^{+0,3}$ & $0,79_{-0,09}^{+0,1}$ & $0,37_{-0,05}^{+0,07}$ & $0,8_{-0,2}^{+0,3}$ & $1,28_{-0,34}^{+0,16}$ & $0,06_{-0,02}^{+0,03}$ & $6,71_{-2,11}^{+1,37}$ & $1,6_{-0,52}^{+0,66}$ & $3,14_{-1,16}^{+1,55}$ & $2,05_{-0,81}^{+1,01}$ \\
\hline & 02 & & & & & $0,8_{-0,2}^{+0,3}$ & $0,9_{-0,37}^{+0,36}$ & $0,03_{-0,01}^{+0,02}$ & $5,31_{-2,32}^{+2,46}$ & $1,19_{-0,52}^{+0,66}$ & $2,31_{-1,05}^{+1,5}$ & $1,65_{-0,73}^{+1,13}$ \\
\hline \multirow[t]{2}{*}{ LMC118.2.27272 } & 01 & $14,0_{-1,5}^{+1,7}$ & $0,1_{-0,1}^{+0,1}$ & $0,73_{-0,08}^{+0,09}$ & $0,73_{-0,08}^{+0,08}$ & $0,5_{-0,1}^{+0,1}$ & $0,24_{-0,17}^{+0,5}$ & $0,12_{-0,07}^{+0,61}$ & $1,29_{-0,87}^{+2,33}$ & $0,14_{-0,08}^{+0,26}$ & $0,21_{-0,13}^{+0,41}$ & $0,11_{-0,06}^{+0,19}$ \\
\hline & & & & & Contint & ua na $\mathrm{pl}$ & xima págir & & & & & \\
\hline
\end{tabular}


Tabela 4.2 - Continuação da página anterior

\begin{tabular}{|c|c|c|c|c|c|c|c|c|c|c|c|c|}
\hline & 02 & & & & & $0,4_{-0,1}^{+0,1}$ & $0,7_{-0,49}^{+0,53}$ & $0,05_{-0,02}^{+0,03}$ & $4,43_{-3,06}^{+3,46}$ & $0,33_{-0,22}^{+0,24}$ & $0,5_{-0,33}^{+0,41}$ & $0,31_{-0,2}^{+0,27}$ \\
\hline \multirow[t]{2}{*}{ LMC118.2.83574 } & 01 & $10,4_{-2,2}^{+2,1}$ & $0,5_{-0,3}^{+0,3}$ & $0,82_{-0,1}^{+0,09}$ & $0,55_{-0,04}^{+0,08}$ & $0,3_{-0,1}^{+0,1}$ & $0,6_{-0,4}^{+0,58}$ & $0,03_{-0,01}^{+0,06}$ & $5,98_{-4,02}^{+5,61}$ & $0,23_{-0,15}^{+0,23}$ & $0,32_{-0,21}^{+0,33}$ & $\overline{0,34_{-0,22}^{+0,35}}$ \\
\hline & 02 & & & & & $0,2_{-0,0}^{+0,1}$ & $0,74_{-0,46}^{+0,49}$ & $0,02_{-0,01}^{+0,01}$ & $8,43_{-5,2}^{+5,82}$ & $0,23_{-0,14}^{+0,15}$ & $0,31_{-0,2}^{+0,23}$ & $0,38_{-0,24}^{+0,28}$ \\
\hline LMC119.2.89739 & 01 & $7,7_{-1,5}^{+1,7}$ & $0,5_{-0,3}^{+0,3}$ & $0,8_{-0,11}^{+0,1}$ & $0,58_{-0,06}^{+0,14}$ & $0,5_{-0,1}^{+0,2}$ & $0,12_{-0,08}^{+0,34}$ & $0,01_{-0,0}^{+0,0}$ & $1,14_{-0,78}^{+3,14}$ & $0,05_{-0,03}^{+0,12}$ & $0,06_{-0,03}^{+0,14}$ & $\overline{0,06_{-0,03}^{+0,12}}$ \\
\hline \multirow[t]{2}{*}{ LMC119.3.41808 } & 01 & $13,6_{-2,9}^{+2,3}$ & $0,3_{-0,2}^{+0,3}$ & $0,81_{-0,11}^{+0,1}$ & $0,33_{-0,02}^{+0,04}$ & $1,7_{-0,4}^{+0,5}$ & $1,06_{-0,28}^{+0,27}$ & $0,15_{-0,03}^{+0,05}$ & $4,18_{-1,09}^{+1,11}$ & $2,55_{-0,85}^{+0,98}$ & $4,17_{-1,49}^{+2,03}$ & $1,83_{-0,66}^{+0,89}$ \\
\hline & 02 & & & & & $1,7_{-0,4}^{+0,5}$ & $1,01_{-0,36}^{+0,33}$ & $0,22_{-0,05}^{+0,05}$ & $3,48_{-1,19}^{+1,08}$ & $2,34_{-0,87}^{+1,07}$ & $3,82_{-1,48}^{+2,12}$ & $1,46_{-0,55}^{+0,8}$ \\
\hline LMC111.7.42828 & 01 & $6,0_{-0,6}^{+0,9}$ & $0,3_{-0,2}^{+0,3}$ & $0,8_{-0,09}^{+0,1}$ & $0,63_{-0,09}^{+0,15}$ & $0,6_{-0,2}^{+0,3}$ & $0,16_{-0,11}^{+0,54}$ & $0,0_{-0,0}^{+0,0}$ & $0,75_{-0,53}^{+2,65}$ & $0,06_{-0,04}^{+0,15}$ & $0,05_{-0,03}^{+0,13}$ & $0,02_{-0,01}^{+0,05}$ \\
\hline \multirow[t]{2}{*}{ LMC110.8.117 } & 01 & $14,1_{-2,0}^{+2,4}$ & $0,7_{-0,2}^{+0,2}$ & $0,84_{-0,11}^{+0,1}$ & $0,54_{-0,03}^{+0,05}$ & $0,5_{-0,1}^{+0,1}$ & $0,04_{-0,01}^{+0,03}$ & $0,03_{-0,01}^{+0,01}$ & $0,51_{-0,16}^{+0,32}$ & $0,04_{-0,02}^{+0,03}$ & $0,09_{-0,03}^{+0,06}$ & $0,14_{-0,05}^{+0,1}$ \\
\hline & 02 & & & & & $0,4_{-0,1}^{+0,1}$ & $0,19_{-0,09}^{+0,35}$ & $0,03_{-0,01}^{+0,02}$ & $1,6_{-0,79}^{+2,86}$ & $0,17_{-0,08}^{+0,28}$ & $0,35_{-0,18}^{+0,56}$ & $0,41_{-0,21}^{+0,64}$ \\
\hline \multirow[t]{2}{*}{ LMC119.7.92903 } & 01 & $14,9_{-2,9}^{+2,2}$ & $0,4_{-0,3}^{+0,3}$ & $0,79_{-0,1}^{+0,11}$ & $0,57_{-0,05}^{+0,08}$ & $0,5_{-0,1}^{+0,1}$ & $1,04_{-0,42}^{+0,32}$ & $0,11_{-0,04}^{+0,21}$ & $4,4_{-1,82}^{+1,47}$ & $0,84_{-0,33}^{+0,31}$ & $1,48_{-0,62}^{+0,64}$ & $\overline{0,72_{-0,3}^{+0,33}}$ \\
\hline & 02 & & & & & $0,6_{-0,1}^{+0,1}$ & $0,8_{-0,5}^{+0,52}$ & $0,06_{-0,02}^{+0,02}$ & $3,62_{-2,09}^{+1,96}$ & $0,66_{-0,37}^{+0,43}$ & $1,17_{-0,66}^{+0,83}$ & $0,59_{-0,3}^{+0,38}$ \\
\hline \multirow[t]{3}{*}{ LMC120.1.40130 } & 01 & $14,8_{-3,0}^{+3,6}$ & $0,6_{-0,3}^{+0,3}$ & $0,8_{-0,1}^{+0,1}$ & $0,02_{-0,02}^{+0,02}$ & $2,9_{-0,4}^{+0,4}$ & $1,02_{-0,39}^{+0,34}$ & $0,68_{-0,18}^{+0,25}$ & $1,02_{-0,42}^{+0,43}$ & $5,42_{-1,64}^{+2,18}$ & $10,81_{-3,47}^{+4,89}$ & $1,47_{-0,55}^{+0,72}$ \\
\hline & 02 & & & & & $1,8_{-0,6}^{+0,8}$ & $0,87_{-0,37}^{+0,42}$ & $1,07_{-0,29}^{+0,28}$ & $1,24_{-0,54}^{+0,71}$ & $2,78_{-0,99}^{+1,57}$ & $5,53_{-2,03}^{+3,41}$ & $1,05_{-0,36}^{+0,56}$ \\
\hline & 03 & & & & & $1,0_{-0,5}^{+0,9}$ & $0,47_{-0,25}^{+0,58}$ & $0,78_{-0,39}^{+0,43}$ & $0,66_{-0,33}^{+0,76}$ & $0,99_{-0,38}^{+0,57}$ & $1,97_{-0,76}^{+1,18}$ & $0,36_{-0,13}^{+0,2}$ \\
\hline \multirow[t]{2}{*}{ LMC120.1.40173 } & 01 & $13,0_{-2,1}^{+2,4}$ & $0,3_{-0,2}^{+0,3}$ & $0,8_{-0,1}^{+0,1}$ & $0,72_{-0,13}^{+0,11}$ & $0,3_{-0,1}^{+0,1}$ & $0,84_{-0,49}^{+0,43}$ & $0,04_{-0,01}^{+0,04}$ & $3,6_{-2,04}^{+1,89}$ & $0,34_{-0,19}^{+0,21}$ & $0,52_{-0,29}^{+0,36}$ & $0,23_{-0,13}^{+0,17}$ \\
\hline & 02 & & & & & $0,4_{-0,1}^{+0,1}$ & $0,82_{-0,49}^{+0,45}$ & $0,04_{-0,02}^{+0,53}$ & $4,88_{-2,87}^{+2,6}$ & $0,37_{-0,22}^{+0,21}$ & $0,56_{-0,32}^{+0,38}$ & $0,35_{-0,2}^{+0,24}$ \\
\hline LMC118.5.53191 & 01 & $6,8_{-1,1}^{+1,2}$ & $0,4_{-0,3}^{+0,3}$ & $0,8_{-0,09}^{+0,1}$ & $0,6_{-0,07}^{+0,15}$ & $0,5_{-0,1}^{+0,1}$ & $0,38_{-0,26}^{+0,58}$ & $0,01_{-0,0}^{+0,0}$ & $4,06_{-2,84}^{+6,57}$ & $0,13_{-0,08}^{+0,18}$ & $0,13_{-0,08}^{+0,18}$ & $0,12_{-0,08}^{+0,18}$ \\
\hline \multirow[t]{2}{*}{ LMC118.2.56865 } & 01 & $11,2_{-1,9}^{+2,6}$ & $0,3_{-0,2}^{+0,2}$ & $0,8_{-0,09}^{+0,1}$ & $0,6_{-0,07}^{+0,09}$ & $0,3_{-0,1}^{+0,4}$ & $0,63_{-0,58}^{+0,54}$ & $0,15_{-0,11}^{+0,83}$ & $3,85_{-3,58}^{+3,49}$ & $0,17_{-0,12}^{+0,18}$ & $0,23_{-0,16}^{+0,26}$ & $0,14_{-0,1}^{+0,16}$ \\
\hline & & & & & Continı & ua na pr & óxima pági & & & & & \\
\hline
\end{tabular}


Tabela 4.2 - Continuação da página anterior

\begin{tabular}{|c|c|c|c|c|c|c|c|c|c|c|c|c|}
\hline & 02 & & & & & $0,3_{-0,1}^{+0,1}$ & $0,6_{-0,38}^{+0,43}$ & $0,27_{-0,19}^{+0,56}$ & $2,56_{-1,61}^{+1,86}$ & $0,2_{-0,11}^{+0,17}$ & $0,28_{-0,15}^{+0,26}$ & $0,12_{-0,06}^{+0,11}$ \\
\hline LMC118.5.23216 & 01 & $10,7_{-2,4}^{+2,0}$ & $0,4_{-0,3}^{+0,3}$ & $0,81_{-0,1}^{+0,1}$ & $0,61_{-0,09}^{+0,17}$ & $0,4_{-0,1}^{+0,1}$ & $0,41_{-0,27}^{+0,57}$ & $0,02_{-0,01}^{+0,02}$ & $9,15_{-6,08}^{+13,02}$ & $0,19_{-0,13}^{+0,24}$ & $0,27_{-0,18}^{+0,35}$ & $0,63_{-0,43}^{+0,82}$ \\
\hline LMC119.3.69304 & 01 & $7,8_{-1,5}^{+1,6}$ & $0,4_{-0,3}^{+0,4}$ & $0,8_{-0,1}^{+0,11}$ & $0,59_{-0,07}^{+0,17}$ & $0,5_{-0,1}^{+0,1}$ & $0,43_{-0,28}^{+0,59}$ & $0,02_{-0,01}^{+0,01}$ & $3,55_{-2,38}^{+5,17}$ & $0,18_{-0,11}^{+0,2}$ & $0,19_{-0,12}^{+0,22}$ & $0,15_{-0,09}^{+0,18}$ \\
\hline \multirow[t]{2}{*}{ LMC119.4.14199 } & 01 & $7,5_{-1,4}^{+2,0}$ & $0,6_{-0,3}^{+0,3}$ & $0,81_{-0,11}^{+0,1}$ & $0,02_{-0,01}^{+0,01}$ & $1,3_{-0,2}^{+0,4}$ & $0,82_{-0,43}^{+0,47}$ & $0,25_{-0,09}^{+0,32}$ & $4,63_{-2,43}^{+2,94}$ & $1,04_{-0,43}^{+0,51}$ & $1,17_{-0,49}^{+0,65}$ & $0,66_{-0,28}^{+0,41}$ \\
\hline & 02 & & & & & $0,8_{-0,1}^{+0,2}$ & $0,41_{-0,2}^{+0,4}$ & $0,14_{-0,04}^{+0,05}$ & $2,94_{-1,35}^{+2,86}$ & $0,36_{-0,15}^{+0,26}$ & $0,41_{-0,18}^{+0,33}$ & $0,29_{-0,12}^{+0,23}$ \\
\hline LMC119.4.66596 & 01 & $14,1_{-1,9}^{+1,8}$ & $0,1_{-0,1}^{+0,2}$ & $0,81_{-0,11}^{+0,11}$ & $0,08_{-0,0}^{+0,0}$ & $3,3_{-1,1}^{+0,1}$ & $0,88_{-0,45}^{+0,46}$ & $0,09_{-0,03}^{+0,04}$ & $3,31_{-1,63}^{+1,93}$ & $2,87_{-1,23}^{+2,44}$ & $4,63_{-2,01}^{+4,33}$ & $1,91_{-0,82}^{+1,63}$ \\
\hline LMC119.6.66150 & 01 & $11,2_{-2,7}^{+2,5}$ & $0,4_{-0,3}^{+0,4}$ & $0,8_{-0,1}^{+0,1}$ & $0,39_{-0,07}^{+0,15}$ & $0,7_{-0,2}^{+0,4}$ & $0,91_{-0,49}^{+0,39}$ & $0,02_{-0,01}^{+0,01}$ & $5,37_{-3,32}^{+2,8}$ & $0,77_{-0,4}^{+0,48}$ & $1,11_{-0,59}^{+0,83}$ & $0,69_{-0,38}^{+0,53}$ \\
\hline LMC118.8.62707 & 01 & $9,5_{-2,1}^{+1,7}$ & $0,4_{-0,3}^{+0,4}$ & $0,81_{-0,11}^{+0,1}$ & $0,02_{-0,02}^{+0,03}$ & $1,8_{-0,6}^{+0,8}$ & $0,6_{-0,37}^{+0,57}$ & $0,07_{-0,02}^{+0,03}$ & $4,84_{-2,86}^{+4,17}$ & $1,06_{-0,51}^{+0,91}$ & $1,35_{-0,66}^{+1,25}$ & $1,11_{-0,53}^{+0,94}$ \\
\hline LMC119.7.66528 & 01 & $5,7_{-0,4}^{+0,8}$ & $0,9_{-0,2}^{+0,1}$ & $0,83_{-0,11}^{+0,1}$ & $0,52_{-0,01}^{+0,03}$ & $0,6_{-0,1}^{+0,1}$ & $0,12_{-0,03}^{+0,06}$ & $0,01_{-0,0}^{+0,0}$ & $0,74_{-0,23}^{+0,38}$ & $0,08_{-0,02}^{+0,04}$ & $0,08_{-0,02}^{+0,04}$ & $0,05_{-0,02}^{+0,03}$ \\
\hline LMC119.6.66163 & 01 & $6,7_{-1,1}^{+1,3}$ & $0,4_{-0,3}^{+0,3}$ & $0,8_{-0,1}^{+0,11}$ & $0,62_{-0,09}^{+0,18}$ & $0,3_{-0,1}^{+0,4}$ & $0,36_{-0,34}^{+0,67}$ & $0,01_{-0,01}^{+0,06}$ & $2,3_{-2,14}^{+4,02}$ & $0,08_{-0,06}^{+0,12}$ & $0,07_{-0,05}^{+0,12}$ & $0,04_{-0,03}^{+0,07}$ \\
\hline LMC119.5.13208 & 01 & $9,9_{-2,2}^{+2,0}$ & $0,4_{-0,3}^{+0,3}$ & $0,8_{-0,1}^{+0,11}$ & $0,61_{-0,08}^{+0,18}$ & $0,3_{-0,1}^{+0,2}$ & $0,48_{-0,43}^{+0,6}$ & $0,02_{-0,01}^{+0,01}$ & $2,66_{-2,41}^{+3,32}$ & $0,16_{-0,12}^{+0,18}$ & $0,21_{-0,16}^{+0,24}$ & $0,12_{-0,09}^{+0,14}$ \\
\hline \multirow[t]{2}{*}{ LMC127.2.18027 } & 01 & $19,8_{-3,0}^{+1,0}$ & $0,7_{-0,1}^{+0,2}$ & $0,83_{-0,1}^{+0,09}$ & $0,01_{-0,01}^{+0,01}$ & $2,4_{-0,5}^{+1,0}$ & $0,75_{-0,43}^{+0,58}$ & $0,47_{-0,09}^{+0,23}$ & $\begin{array}{r}1,69_{-0,99}^{+1,38}\end{array}$ & $5,03_{-2,08}^{+2,67}$ & $13,01_{-5,47}^{+7,42}$ & $\begin{array}{r}4,57_{-1,94}^{+2,73} \\
\end{array}$ \\
\hline & 02 & & & & & $3,3_{-0,4}^{+0,1}$ & $0,38_{-0,06}^{+0,13}$ & $0,64_{-0,12}^{+0,14}$ & $0,54_{-0,08}^{+0,16}$ & $3,66_{-0,93}^{+1,47}$ & $9,6_{-2,88}^{+4,58}$ & $2,23_{-0,67}^{+1,06}$ \\
\hline LMC126.1.48828 & 01 & $11,7_{-2,4}^{+2,2}$ & $0,5_{-0,3}^{+0,3}$ & $0,82_{-0,11}^{+0,1}$ & $\begin{array}{r}0,35_{-0,03}^{+0,09} \\
\end{array}$ & $2,2_{-0,7}^{+0,9}$ & $0,6_{-0,28}^{+0,43}$ & $0,03_{-0,01}^{+0,02}$ & $5,79_{-2,57}^{+3,96}$ & $1,92_{-1,1}^{+1,91}$ & $2,94_{-1,7}^{+3,4}$ & $3,16_{-1,77}^{+3,68}$ \\
\hline LMC127.1.38725 & 01 & $17,5_{-4,1}^{+2,5}$ & $0,6_{-0,3}^{+0,3}$ & $0,81_{-0,1}^{+0,1}$ & $0,01_{-0,01}^{+0,01}$ & $1,8_{-0,4}^{+0,5}$ & $0,69_{-0,22}^{+0,44}$ & $\begin{array}{r}0,64_{-0,12}^{+0,18} \\
\end{array}$ & $3,5_{-1,08}^{+2,32}$ & $2,8_{-0,79}^{+1,18}$ & $\begin{array}{r}6,14_{-1,88}^{+2,99} \\
\end{array}$ & $\begin{array}{r}4,38_{-1,37}^{+2,18} \\
\end{array}$ \\
\hline LMC127.4.10051 & 01 & $7,9_{-1,0}^{+1,0}$ & $0,1_{-0,1}^{+0,2}$ & $0,78_{-0,1}^{+0,11}$ & $0,74_{-0,11}^{+0,14}$ & $0,5_{-0,1}^{+0,1}$ & $0,29_{-0,19}^{+0,51}$ & $0,0_{-0,0}^{+0,0}$ & $\begin{array}{r}3,23_{-2,15}^{+5,88} \\
\end{array}$ & $0,11_{-0,06}^{+0,18}$ & $0,11_{-0,07}^{+0,18}$ & $0,1_{-0,06}^{+0,18}$ \\
\hline LMC170.3.43578 & 01 & $6,0_{-0,7}^{+1,2}$ & $0,8_{-0,3}^{+0,2}$ & $0,82_{-0,1}^{+0,1}$ & $0,52_{-0,02}^{+0,04}$ & $0,8_{-0,1}^{+0,2}$ & $0,07_{-0,03}^{+0,04}$ & $\begin{array}{r}0,02_{-0,0}^{+0,0} \\
\end{array}$ & $1,23_{-0,47}^{+0,69}$ & $\begin{array}{r}0,06_{-0,02}^{+0,03} \\
\end{array}$ & $0,06_{-0,02}^{+0,03}$ & $0,09_{-0,03}^{+0,05}$ \\
\hline LMC177.5.17601 & 01 & $7,4_{-0,9}^{+2,2}$ & $0,8_{-0,3}^{+0,2}$ & $0,83_{-0,11}^{+0,1}$ & $0,54_{-0,03}^{+0,1}$ & $0,5_{-0,1}^{+0,2}$ & $0,03_{-0,02}^{+0,05}$ & $0,01_{-0,0}^{+0,0}$ & $0,36_{-0,19}^{+0,53}$ & $0,02_{-0,01}^{+0,02}$ & $0,03_{-0,01}^{+0,03}$ & $\begin{array}{r}0,03_{-0,02}^{+0,03} \\
\end{array}$ \\
\hline LMC176.6.30752 & 01 & $20,8_{-0,4}^{+0,2}$ & $0,1_{-0,1}^{+0,1}$ & $0,75_{-0,09}^{+0,1}$ & $0,72_{-0,02}^{+0,02}$ & $1,5_{-0,1}^{+0,1}$ & $0,99_{-0,17}^{+0,19}$ & $0,09_{-0,01}^{+0,01}$ & $3,78_{-0,69}^{+0,72}$ & $2,37_{-0,41}^{+0,44}$ & $5,15_{-0,96}^{+1,09}$ & $2,36_{-0,44}^{+0,51}$ \\
\hline
\end{tabular}


Tabela 4.2 - Continuação da página anterior

\begin{tabular}{|c|c|c|c|c|c|c|c|c|c|c|c|c|}
\hline & 02 & & & & & $1,5_{-0,1}^{+0,1}$ & $1,03_{-0,23}^{+0,25}$ & $0,06_{-0,01}^{+0,01}$ & $3,28_{-0,72}^{+0,78}$ & $2,39_{-0,54}^{+0,59}$ & $5,21_{-1,22}^{+1,36}$ & $1,99_{-0,46}^{+0,51}$ \\
\hline & 03 & & & & & $1,8_{-0,1}^{+0,1}$ & $0,34_{-0,1}^{+0,24}$ & $0,15_{-0,04}^{+0,05}$ & $1,37_{-0,41}^{+1,0}$ & $0,95_{-0,25}^{+0,56}$ & $2,09_{-0,58}^{+1,23}$ & $1,01_{-0,29}^{+0,59}$ \\
\hline & 04 & & & & & $1,5_{-0,1}^{+0,1}$ & $0,78_{-0,21}^{+0,24}$ & $0,09_{-0,01}^{+0,01}$ & $2,14_{-0,61}^{+0,69}$ & $1,86_{-0,47}^{+0,56}$ & $4,05_{-1,06}^{+1,28}$ & $1,34_{-0,35}^{+0,43}$ \\
\hline LMC176.8.82613 & 01 & $5,8_{-0,6}^{+0,8}$ & $0,4_{-0,3}^{+0,3}$ & $0,79_{-0,1}^{+0,11}$ & $0,57_{-0,06}^{+0,17}$ & $0,4_{-0,1}^{+0,1}$ & $0,66_{-0,45}^{+0,56}$ & $0,04_{-0,02}^{+0,02}$ & $6,16_{-4,17}^{+5,08}$ & $0,17_{-0,11}^{+0,15}$ & $0,14_{-0,09}^{+0,13}$ & $0,11_{-0,07}^{+0,1}$ \\
\hline \multirow[t]{2}{*}{ LMC177.3.50735 } & 01 & $11,0_{-2,5}^{+1,5}$ & $0,3_{-0,2}^{+0,3}$ & $0,81_{-0,1}^{+0,1}$ & $0,04_{-0,02}^{+0,02}$ & $2,6_{-0,6}^{+0,6}$ & $0,88_{-0,44}^{+0,42}$ & $0,52_{-0,2}^{+0,3}$ & $3,19_{-1,62}^{+1,82}$ & $2,53_{-1,15}^{+1,29}$ & $3,54_{-1,6}^{+1,95}$ & $1,32_{-0,6}^{+0,85}$ \\
\hline & 03 & & & & & $2,0_{-0,6}^{+0,7}$ & $0,86_{-0,48}^{+0,43}$ & $0,2_{-0,09}^{+0,16}$ & $4,36_{-2,36}^{+2,43}$ & $1,86_{-0,98}^{+1,31}$ & $2,64_{-1,41}^{+1,84}$ & $1,37_{-0,66}^{+1,02}$ \\
\hline \multirow[t]{2}{*}{ LMC177.6.90276 } & 01 & $14,7_{-3,4}^{+3,0}$ & $0,4_{-0,3}^{+0,3}$ & $0,81_{-0,1}^{+0,1}$ & $0,57_{-0,05}^{+0,09}$ & $0,6_{-0,1}^{+0,2}$ & $1,19_{-0,36}^{+0,23}$ & $0,06_{-0,02}^{+0,02}$ & $5,85_{-1,79}^{+1,3}$ & $1,18_{-0,39}^{+0,38}$ & $2,16_{-0,77}^{+0,9}$ & $1,29_{-0,46}^{+0,54}$ \\
\hline & 02 & & & & & $0,7_{-0,1}^{+0,2}$ & $0,2_{-0,09}^{+0,26}$ & $0,05_{-0,01}^{+0,02}$ & $1,47_{-0,63}^{+1,86}$ & $0,23_{-0,1}^{+0,27}$ & $0,43_{-0,2}^{+0,52}$ & $0,39_{-0,18}^{+0,47}$ \\
\hline LMC177.2.11525 & 01 & $\begin{array}{r}6,3_{-0,8}^{+0,9} \\
\end{array}$ & $0,2_{-0,2}^{+0,3}$ & $0,79_{-0,1}^{+0,11}$ & $0,69_{-0,13}^{+0,15}$ & $0,4_{-0,1}^{+0,1}$ & $0,3_{-0,21}^{+0,58}$ & $0,06_{-0,03}^{+0,28}$ & $5,17_{-3,62}^{+9,87}$ & $0,07_{-0,05}^{+0,13}$ & $0,06_{-0,04}^{+0,11}$ & $0,09_{-0,06}^{+0,15}$ \\
\hline \multirow[t]{2}{*}{ LMC176.1.18156 } & 01 & $8,2_{-1,5}^{+1,8}$ & $0,6_{-0,3}^{+0,3}$ & $0,81_{-0,11}^{+0,09}$ & $0,54_{-0,03}^{+0,06}$ & $0,9_{-0,2}^{+0,2}$ & $0,96_{-0,35}^{+0,37}$ & $0,05_{-0,01}^{+0,01}$ & $3,43_{-1,22}^{+1,4}$ & $0,95_{-0,33}^{+0,4}$ & $1,13_{-0,42}^{+0,57}$ & $0,41_{-0,15}^{+0,21}$ \\
\hline & 02 & & & & & $0,7_{-0,1}^{+0,2}$ & $1,15_{-0,33}^{+0,24}$ & $0,08_{-0,02}^{+0,02}$ & $7,68_{-2,15}^{+1,74}$ & $0,96_{-0,3}^{+0,31}$ & $1,15_{-0,39}^{+0,47}$ & $0,78_{-0,27}^{+0,32}$ \\
\hline LMC185.8.314 & 01 & $7,7_{-1,3}^{+1,1}$ & $0,2_{-0,2}^{+0,3}$ & $0,79_{-0,1}^{+0,1}$ & $0,71_{-0,12}^{+0,14}$ & $0,7_{-0,2}^{+0,4}$ & $0,18_{-0,12}^{+0,62}$ & $0,02_{-0,01}^{+0,02}$ & $0,65_{-0,44}^{+2,29}$ & $0,11_{-0,06}^{+0,23}$ & $0,12_{-0,07}^{+0,24}$ & $0,04_{-0,02}^{+0,08}$ \\
\hline \multirow[t]{2}{*}{ LMC185.5.241 } & 01 & $7,1_{-1,2}^{+1,2}$ & $0,3_{-0,2}^{+0,4}$ & $0,82_{-0,11}^{+0,1}$ & $0,32_{-0,02}^{+0,04}$ & $0,8_{-0,1}^{+0,2}$ & $0,87_{-0,39}^{+0,4}$ & $0,05_{-0,01}^{+0,02}$ & $7,64_{-3,46}^{+3,6}$ & $0,56_{-0,23}^{+0,28}$ & $0,55_{-0,23}^{+0,3}$ & $0,43_{-0,18}^{+0,24}$ \\
\hline & 02 & & & & & $0,9_{-0,2}^{+0,2}$ & $0,66_{-0,35}^{+0,49}$ & $0,02_{-0,01}^{+0,01}$ & $5,77_{-3,11}^{+4,4}$ & $0,49_{-0,25}^{+0,34}$ & $0,49_{-0,25}^{+0,35}$ & $0,37_{-0,18}^{+0,28}$ \\
\hline
\end{tabular}


Capítulo 5

\section{Conclusões}

Aplicamos a metodologia de modelagem de curvas de luz desenvolvida por Rímulo et al. (2018), cujo objetivo é obter estimativas de parâmetros estelares $\left(M, t / t_{\mathrm{ms}}\right.$ e $\left.W\right)$, geométrico $(\cos i)$ e de disco $\left(\Sigma_{0}, \alpha_{\mathrm{bu}}\right.$ e $\left.\alpha_{\mathrm{d}}\right)$. O método utiliza uma grade extensa de curvas de luz sintéticas, combinando cálculos hidrodinâmicos e radiativos. Para a comparação entre dados observacionais e modelos utilizamos uma técnica de MCMC para estimar a probabilidade posterior dos parâmetros ajustados.

Uma amostra de candidatas a estrelas Be da LMC (Sabogal et al., 2005), contendo 83 estrelas e 120 bumps, foi modelada. As curvas de luz reúnem dados das fases II, III e IV do levantamento OGLE, provendo uma base temporal de 18 anos. Identificamos em nossa amostra vários bumps (26) de duração extensa, que denominamos VLBs (very long bumps). Não identificamos nenhum relato desse tipo de fenômeno na literatura. Somado ao grande número de ocorrências pode sugerir um comportamento distinto dos discos de estrelas Be da LMC em comparação aos da SMC ou da Galáxia. Também realizamos um estudo inicial das propriedades estatísticas dos bumps, além de uma análise preliminar visando identificar a existência de periodicidades nos dados. Este tipo de análise se mostrou promissora, porém um estudo mais detalhado ainda é necessário.

Em nosso estudo fizemos uso da grade de curvas de luz sintéticas computada por Rímulo et al. (2018) para a $\operatorname{SMC}(Z=0,002)$. Esta é uma inconsistência que será remediada antes da publicação final dos resultados. Visando aprimorar a futura grade de modelos para a LMC, exploramos os efeitos da idade e rotação estelar na amplitude do bump, o que mostrou que ambos fatores têm impacto sobre a curva de luz. Assim sendo, a grade de modelos para a LMC levará em conta esses parâmetros.

A partir do diagrama de cor-magnitude da amostra de estrelas (Fig. 3.4), nota-se que 
a maioria da amostra se encontra à direita da região correspondente à Sequência principal, ocupada pelos modelos fotosféricos, o que indica que o avermelhamento empregado está possivelmente incorreto. Faz-se necessário, assim, um estudo mais aprofundado deste parâmetro antes da publicação final dos resultados.

Obtivemos que a nossa amostra de estrelas contém mais estrelas recentes que tardias. Estrelas recentes são tipicamente mais ativas e possuem discos mais densos, o que gera bumps mais claros. Adicionalmente, os critérios de seleção dos bumps (bem definidos e excluindo eventos de curta duração e intensidade) privilegia eventos cujo disco possui maior densidade. Nossa amostra deve, desta maneira, ser representativa dos discos mais densos para estrelas Be da LMC. Encontramos um aumento de $\Sigma_{0}$ com a massa da estrela, o que também foi observado no estudo de estrelas Be da SMC (Rímulo et al., 2018), e a mediana da nossa amostra é de $\left\langle\Sigma_{0}\right\rangle=0,63_{-0,28}^{+1,09} \mathrm{~g} \mathrm{~cm}^{-2}$, sendo menor do que o valor encontrado por Rímulo et al. que corresponde a $\left\langle\Sigma_{0}\right\rangle=1,50_{-0,83}^{+1,12} \mathrm{~g} \mathrm{~cm}^{-2}$.

Os eventos de disco são mais curtos para as estrelas mais massivas, indicando que estas são mais ativas em acordo com resultados para a Galáxia e para a SMC. A mediana desse tempo de construção é de $\left\langle t_{2}-t_{1}\right\rangle=635_{-3,04}^{+6,86}$ dias, valor aproximadamente duas vezes maior do que o obtido para a SMC, que é de $\left\langle t_{2}-t_{1}\right\rangle=304_{-168}^{+351}$ dias.

Em nossa análise, permitimos que o valor da viscosidade variasse entre as fases de construção e dissipação do disco. Encontramos evidências que sugerem uma correlação entre os parâmetros $\alpha_{\mathrm{bu}}$ e $\alpha_{\mathrm{d}} \operatorname{com} M$. A mediana encontrada para esses valores são de $0,59_{-0,49}^{+0,58}$ e $0,06_{-0,05}^{+0,40}$, respectivamente. Embora menores, os valores para $\alpha_{\text {bu }}$ ainda são compatíveis com resultados obtidos para a SMC $\left(\left\langle\alpha_{\mathrm{bu}}\right\rangle=0,64_{-0,38}^{+0,50}\right.$ e $\left.\left\langle\alpha_{\mathrm{d}}\right\rangle=0,26_{-0,18}^{+0,60}\right)$, para a estrela Be Galática 28 CMa (Carciofi et al. 2012; Ghoreyshi e Carciofi 2017) e estimativas encontradas para variáveis cataclísmicas (King et al. 2007; Kotko e Lasota 2012). Entretanto, nossos resultados para $\alpha_{\mathrm{d}}\left(0,06_{-0,05}^{+0,40}\right)$ são 10 vezes menores que os reportados para a SMC. Este resultado está em harmonia com a identificação dos VLB, que seriam explicados de forma simples pela baixa viscosidade do disco, que tem como consequência um elevado tempo de dissipação.

O resultado de $\alpha_{\mathrm{bu}}$ maior do que $\alpha_{\mathrm{d}}$ também foi obtido por Rímulo et al. (2018) e Ghoreyshi e Carciofi (2017) para os vários ciclos de atividade de 28 CMa. Porém, estudos mais aprofundados são necessários para determinar se esse efeito é real ou apenas um resultado das suposições do modelo. Há dois principais efeitos que não foram considerados 
nesse trabalho, são eles: o fato do disco não ser isotérmico e forças radiativas. Espera-se que as consequências desse último não devam ser de grande relevância para estrelas da LMC e SMC, em consequência de suas metalicidades baixas. Outra explicação possível para $\alpha_{\mathrm{bu}}>\alpha_{\mathrm{d}}$ é o fato da injeção de massa ser um processo mecanicamente mais brusco que a dissipação.

A taxa de perda de massa e momento angular para os discos mais densos, considerando um regime de estado estacionário, é da ordem de $\sim 10^{-10} M_{\odot} \mathrm{ano}^{-1} \mathrm{e} \sim 10^{37} \mathrm{~g} \mathrm{~cm}^{2} \mathrm{~s}^{-1}$. A taxa de perda de massa é compatível com estimativas feitas por Vieira et al. (2017), que estudaram uma amostra de 80 estrelas Be da Galáxia. Os valores obtidos para a taxa de perda de massa são compatíveis com os derivados para estrelas da SMC, $\sim 10^{-10} M_{\odot}$ ano $^{-1}$. A estimativa da taxa de perda de massa aproximadamente difere em uma ordem de grandeza do valor obtido para a amostra da SMC que é de $\sim 5 \times 10^{36} \mathrm{~g} \mathrm{~cm}^{2} \mathrm{~s}^{-1}$ (Rímulo et al., 2018). Potencialmente, esse pode ser um indício de que a metalicidade também altera os valores de $(-\partial M / \partial t)_{\text {est }}$ e $(-\partial J / \partial t)$. Para a taxa de perda de momento angular temos que nossas estimativas são, aproximadamente, uma ordem de grandeza menor do que a quantidade prevista pelos modelos de evolutivos do grupo de Genebra de maneira a manter a estrela em um regime de rotação subcrítico. Esta inconsistência, reportada aqui pela primeira vez para estrelas da LMC, também foi encontrada para estrelas da SMC (Rímulo et al., 2018) e para uma estrela da Galáxia (28 CMa, Ghoreyshi et al. 2018). 


\section{Capítulo 6}

\section{Perspectivas}

O presente trabalho possui perspectivas teóricas e observacionais.

Pelo lado observacional, a extensão mais evidente é a modelagem de curvas de luz para estrelas Be da Galáxia. Esperamos que isso seja feito nos próximos anos em parceria com Jonathan Labadie-Bartz ${ }^{1}$, que possui uma amostra de curvas de luz de estrelas Be.

Outro ponto importante é o desenvolvimento de uma análise estatística das curvas de luz que possuímos, com o objetivo de extrair propriedades fundamentais do fenômeno Be, de forma geral, e também do bumps. No capítulo 3 mostramos alguns resultados preliminares, mas no início do doutorado esta análise, que tem a vantagem de ser independente de modelos teóricos, será aprofundada e os resultados publicados separadamente.

Uma melhor determinação dos parâmetros estelares também é de grande importância para melhor a estimativa dos parâmetros modelados. Para tanto, utilizaremos dados observacionais coletados com o telescópio SOAR, para estrelas Be da LMC e da SMC que se encontram em aglomerados abertos. Utilizaremos medidas fotométricas para o ajuste de isócronas para uma melhor determinação da idade estelar. Através da espectroscopia, teremos uma melhor determinação do tipo espectral estelar (ou seja, uma melhor determinação de $M$ ) e uma estimativa da velocidade de rotação. No futuro próximo, teremos disponíveis dados polarimétricos no visível, de banda larga, graças ao levantamento SOUTH-POL, que imageará todo o céu do hemisfério Sul, a partir de Cerro Tololo ${ }^{2}$. A polarimetria nos fornecerá informações sobre densidade, nas regiões internas do disco, e também auxiliará na restrição do ângulo de inclinação. Para a publicação final dos modelos das curvas de luz da LMC será computada uma nova grade de modelos usando os parâmetros estelares

\footnotetext{
${ }^{1}$ Integrante do time KELT.

2 Cerro Tololo Inter-American Observatório, o CTIO.
} 
apropriados à LMC.

No panorama teórico, uma das perspectivas futuras mais importantes é um tratamento de disco não isotérmico. A implementação de um tratamento não isotérmico será liderada pelo Dr. Leandro Rocha Rímulo, em colaboração conosco.

Outra questão importante é a análise do efeito de forças radiativas na descrição de disco viscoso empregada nesse trabalho, especialmente para o ambiente Galático. Em particular, devemos entender como a ablação, como sugerida por Kee et al. (2016) ou Krtička et al. (2011) deve afetar os processos de construção e dissipação de discos de estrelas Be e, por conseguinte, a determinação do parâmetro de viscosidade

Este trabalho também contribuirá com estimativas de taxas de perda de momento angular. Nossas estimativas iniciais indicam que os valores obtidos para a amostra de estrelas Be da LMC estão abaixo dos valores previstos pelos modelos de evolução estelar de Genebra (Granada et al., 2013) para que as estrelas não atinjam rotações críticas durante a sua estadia na Sequência principal. Com o acréscimo do número de estrelas Be com essa quantidade determinada, esperamos colaborar com o grupo de Genebra testando seus modelos evolucionários no futuro. 


\section{Referências Bibliográficas}

Aerts C., Christensen-Dalsgaard J., Kurtz D. W., Asteroseismology. SPRINGER, 2010

Alcock C., Allsman R. A., Alves D., Axelrod T. S., Becker A. C., Bennett D. P., Cook K. H., Freeman K. C., Griest K., Guern J., Lehner M. J., Marshall S. L., Peterson B. A., Pratt M. R., Quinn P. J., Rodgers A. W., Stubbs C. W., Sutherland W., Welch D. L., The MACHO Project Large Magellanic Cloud Microlensing Results from the First Two Years and the Nature of the Galactic Dark Halo, ApJ, 1997, vol. 486, p. 697

Andrews S. M., Wilner D. J., Zhu Z., Birnstiel T., Carpenter J. M., Pérez L. M., Bai X.-N., Öberg K. I., Hughes A. M., Isella A., Ricci L., Ringed Substructure and a Gap at $1 \mathrm{au}$ in the Nearest Protoplanetary Disk, ApJ, 2016, vol. 820, p. L40

Aubourg E., Bareyre P., Bréhin S., Gros M., Lachièze-Rey M., Laurent B., Lesquoy E., Magneville C., Milsztajn A., Moscoso L., Queinnec F., Rich J., et al. Evidence for gravitational microlensing by dark objects in the Galactic halo, Nature, 1993, vol. 365, p. 623

Baade D., Nonradial Pulsations in Relation to Wind Variability in Early-Type Stars. In Cyclical Variability in Stellar Winds, 1998, p. 196

Baade D., Rivinius T., Pigulski A., Carciofi A. C., Martayan C., Moffat A. F. J., Wade G. A., Weiss W. W., Grunhut J., Handler G., Kuschnig R., Mehner A., Pablo H., Popowicz A., Rucinski S., Whittaker G., Short-term variability and mass loss in Be stars. I. BRITE satellite photometry of $\eta$ and $\mu$ Centauri, A\&A, 2016, vol. 588, p. A56

Baade D., Rivinius T., Štefl S., Nonradial Pulsations and Magnetic Fields in Be Stars. In Magnetic Fields in O, B and A Stars: Origin and Connection to Pulsation, Rotation 
and Mass Loss , vol. 305 of Astronomical Society of the Pacific Conference Series, 2003, p. 269

Bednarski D., Polarimetria de estrelas Be Próximas, Iag-Usp Oca-Uns, 2016, Tese de Doutorado

Bessell M. S., UBVRI passbands, PASP, 1990, vol. 102, p. 1181

Bjorkman J. E., Circumstellar Disks. In Stellar Atmospheres: Theory and Observations , vol. 497 of Lecture Notes in Physics, Berlin Springer Verlag, 1997, p. 239

Bjorkman J. E., Carciofi A. C., Modeling the Structure of Hot Star Disks. In The Nature and Evolution of Disks Around Hot Stars , vol. 337 of Astronomical Society of the Pacific Conference Series, 2005, p. 75

Carciofi A. C., The circumstellar discs of Be stars. In Active OB Stars: Structure, Evolution, Mass Loss, and Critical Limits , vol. 272 of IAU Symposium, 2011, p. 325

Carciofi A. C., Bjorkman J. E., Non-LTE Monte Carlo Radiative Transfer. I. The Thermal Properties of Keplerian Disks around Classical Be Stars, ApJ, 2006, vol. 639, p. 1081

Carciofi A. C., Bjorkman J. E., Non-LTE Monte Carlo Radiative Transfer. II. Nonisothermal Solutions for Viscous Keplerian Disks, ApJ, 2008, vol. 684, p. 1374

Carciofi A. C., Bjorkman J. E., Otero S. A., Okazaki A. T., Štefl S., Rivinius T., Baade D., Haubois X., The First Determination of the Viscosity Parameter in the Circumstellar Disk of a Be Star, ApJ, 2012, vol. 744, p. L15

Carciofi A. C., Domiciano de Souza A., Magalhães A. M., Bjorkman J. E., Vakili F., On the Determination of the Rotational Oblateness of Achernar, ApJ, 2008, vol. 676, p. L41

Carciofi A. C., Magalhães A. M., Leister N. V., Bjorkman J. E., Levenhagen R. S., Achernar: Rapid Polarization Variability as Evidence of Photospheric and Circumstellar Activity, ApJ, 2007, vol. 671, p. L49

Carciofi A. C., Miroshnichenko A. S., Bjorkman J. E., Toward Understanding the B[e] Phenomenon. IV. Modeling of IRAS 00470+6429, ApJ, 2010, vol. 721, p. 1079 
Carciofi A. C., Miroshnichenko A. S., Kusakin A. V., Bjorkman J. E., Bjorkman K. S., Marang F., Kuratov K. S., García-Lario P., Calderón J. V. P., Fabregat J., Magalhães A. M., Properties of the $\delta$ Scorpii Circumstellar Disk from Continuum Modeling, ApJ, 2006, vol. 652, p. 1617

Carciofi A. C., Okazaki A. T., Le Bouquin J.-B., Štefl S., Rivinius T., Baade D., Bjorkman J. E., Hummel C. A., Cyclic variability of the circumstellar disk of the Be star $\zeta$ Tauri. II. Testing the 2D global disk oscillation model, A\&A, 2009, vol. 504, p. 915

Collins II G. W., The use of terms and definitions in the study of Be stars. In IAU Colloq. 92: Physics of Be Stars , 1987, p. 3

Cox A. N., Allen's astrophysical quantities, 2000

Cranmer S. R., A Statistical Study of Threshold Rotation Rates for the Formation of Disks around Be Stars, ApJ, 2005, vol. 634, p. 585

Domiciano de Souza A., Hadjara M., Vakili F., Bendjoya P., Millour F., Abe L., Carciofi A. C., Faes D. M., Kervella P., Lagarde S., Marconi A., Monin J.-L., Niccolini G., Petrov R. G., Weigelt G., Beyond the diffraction limit of optical/IR interferometers. I. Angular diameter and rotation parameters of Achernar from differential phases, A\&A, 2012, vol. 545, p. A130

Domiciano de Souza A., Kervella P., Jankov S., Abe L., Vakili F., di Folco E., Paresce F., The spinning-top Be star Achernar from VLTI-VINCI, A\&A, 2003, vol. 407, p. L47

Escolano C., Carciofi A. C., Okazaki A. T., Rivinius T., Baade D., Štefl S., 2.5D globaldisk oscillation models of the Be shell star $\zeta$ Tauri. I. Spectroscopic and polarimetric analysis, A\&A, 2015, vol. 576, p. A112

Espinosa Lara F., Rieutord M., Gravity darkening in rotating stars, A\&A, 2011, vol. 533, p. A43

Faes D. M., Carciofi A. C., Rivinius T., Štefl S., Baade D., Domiciano de Souza A., Differential interferometric phases at high spectral resolution as a sensitive physical diagnostic of circumstellar disks, A\&A, 2013, vol. 555, p. A76 
Fish V. L., Johnson M. D., Doeleman S. S., Broderick A. E., Psaltis D., Lu R.-S., Akiyama K., Alef W., Algaba J. C., Asada K., Beaudoin C., et al. VizieR Online Data Catalog: 4yr 1.3mm VLBI observations of SgrA* with EHT (Fish+, 2016), VizieR Online Data Catalog, 2016, vol. 182

Foreman-Mackey D., Hogg D. W., Lang D., Goodman J., emcee: The MCMC Hammer, PASP, 2013, vol. 125, p. 306

Frémat Y., Zorec J., Hubert A.-M., Floquet M., Effects of gravitational darkening on the determination of fundamental parameters in fast-rotating B-type stars, A\&A, 2005, vol. 440 , p. 305

Friaça A. C. S., Dal Pino E., Jatenco-Pereira V., Sodré Jr. L., Astronomia: uma visão geral do universo. EDUSP, 2003

Georgy C., Ekström S., Eggenberger P., Meynet G., Haemmerlé L., Maeder A., Granada A., Groh J. H., Hirschi R., Mowlavi N., Yusof N., Charbonnel C., Decressin T., Barblan F., Grids of stellar models with rotation. III. Models from 0.8 to $120 \mathrm{M}_{\odot}$ at a metallicity $\mathrm{Z}=0.002, \mathrm{~A} \& \mathrm{~A}, 2013$, vol. 558, p. A103

Georgy C., Ekström S., Granada A., Meynet G., Mowlavi N., Eggenberger P., Maeder A., Populations of rotating stars. I. Models from 1.7 to $15 \mathrm{M}_{\text {sun; }}$ at $\mathrm{Z}=0.014,0.006$, and 0.002 with $\Omega / \Omega_{\text {crit }}$ between 0 and $1, \mathrm{~A} \& \mathrm{~A}, 2013$, vol. 553, p. A24

Ghoreyshi M. R., Carciofi A. C., Analysis of the V-Band Light Curve of the Be Star $\omega$ CMa with the Viscous Decretion Disk Model. In The B[e] Phenomenon: Forty Years of Studies, vol. 508 of Astronomical Society of the Pacific Conference Series, 2017, p. 323

Ghoreyshi M. R., Carciofi A. C., Rímulo L. R., Vieira R. G., Faes D. M., Baade D., Bjorkman J. E., Otero S., Rivinius T., The life cycles of Be viscous decretion discs: The case of $\omega$ CMa, MNRAS, 2018, vol. 479, p. 2214

Gordon K. D., Clayton G. C., Misselt K. A., Landolt A. U., Wolff M. J., A Quantitative Comparison of the Small Magellanic Cloud, Large Magellanic Cloud, and Milky Way Ultraviolet to Near-Infrared Extinction Curves, ApJ, 2003, vol. 594, p. 279 
Granada A., Ekström S., Georgy C., Krtička J., Owocki S., Meynet G., Maeder A., Populations of rotating stars. II. Rapid rotators and their link to Be-type stars, A\&A, 2013, vol. 553 , p. A25

Hanuschik R. W., Shell lines in disks around Be stars. 1: Simple approximations for Keplerian disks, A\&A, 1995, vol. 295, p. 423

Haschke R., Grebel E. K., Duffau S., New Optical Reddening Maps of the Large and Small Magellanic Clouds, AJ, 2011, vol. 141, p. 158

Haubois X., Carciofi A. C., Rivinius T., Okazaki A. T., Bjorkman J. E., Dynamical Evolution of Viscous Disks around Be Stars. I. Photometry, ApJ, 2012, vol. 756, p. 156

Haubois X., Mota B. C., Carciofi A. C., Draper Z. H., Wisniewski J. P., Bednarski D., Rivinius T., Dynamical Evolution of Viscous Disks around Be Stars. II. Polarimetry, ApJ, 2014, vol. 785, p. 12

Jaschek M., Slettebak A., Jaschek C., , 1981 Be star terminology. Be Star Newsletter

Jones C. E., Sigut T. A. A., Marlborough J. M., Iron line cooling of Be star circumstellar discs, MNRAS, 2004, vol. 352, p. 841

Jones C. E., Sigut T. A. A., Porter J. M., The circumstellar envelopes of Be stars: viscous disc dynamics, MNRAS, 2008, vol. 386, p. 1922

Kee N. D., Owocki S., Sundqvist J. O., Line-driven ablation of circumstellar discs - I. Optically thin decretion discs of classical Oe/Be stars, MNRAS, 2016, vol. 458, p. 2323

Keller S. C., Bessell M. S., Cook K. H., Geha M., Syphers D., Blue Variable Stars from the MACHO Database. I. Photometry and Spectroscopy of the Large Magellanic Cloud Sample, AJ, 2002, vol. 124, p. 2039

Kepler de Oliveira Astronomia \& Astrofísica (Em Portuguese do Brasil). Livraria da Física, 2013

King A. R., Pringle J. E., Livio M., Accretion disc viscosity: how big is alpha?, MNRAS, 2007, vol. 376, p. 1740 
Klement R., Carciofi A. C., Rivinius T., Matthews L. D., Vieira R. G., Ignace R., Bjorkman J. E., Mota B. C., Faes D. M., Bratcher A. D., Curé M., Štefl S., Revealing the structure of the outer disks of Be stars, A\&A, 2017, vol. 601, p. A74

Klement R., Carciofi A. C., Rivinius T., Panoglou D., Vieira R. G., Bjorkman J. E., Štefl S., Tycner C., Faes D. M., Korčáková D., Müller A., Zavala R. T., Curé M., Multitechnique testing of the viscous decretion disk model. I. The stable and tenuous disk of the late-type Be star $\beta$ CMi, A\&A, 2015, vol. 584, p. A85

Kotko I., Lasota J.-P., The viscosity parameter $\alpha$ and the properties of accretion disc outbursts in close binaries, A\&A, 2012, vol. 545, p. A115

Kraus S., Monnier J. D., Che X., Schaefer G., Touhami Y., Gies D. R., Aufdenberg J. P., Baron F., Thureau N., ten Brummelaar T. A., McAlister H. A., Turner N. H., Sturmann J., Sturmann L., Gas Distribution, Kinematics, and Excitation Structure in the Disks around the Classical Be Stars $\beta$ Canis Minoris and $\zeta$ Tauri, ApJ, 2012, vol. 744, p. 19

Kroupa P., On the variation of the initial mass function, MNRAS, 2001, vol. 322, p. 231

Krtička J., Owocki S. P., Meynet G., Mass and angular momentum loss via decretion disks, A\&A, 2011, vol. 527, p. A84

Labadie-Bartz J., Chojnowski S. D., Whelan D. G., Pepper J., McSwain M. V., Borges Fernandes M., Wisniewski J. P., Stringfellow G. S., Carciofi A. C., Siverd e. a., Outbursts and Disk Variability in Be Stars, AJ, 2018, vol. 155, p. 53

Labadie-Bartz J., Pepper J., McSwain M. V., Bjorkman J. E., Bjorkman K. S., Lund M. B., Rodriguez J. E., Stassun K. G., Stevens D. J., James D. J., Kuhn R. B., Siverd R. J., Beatty T. G., Photometric Variability of the Be Star Population, AJ, 2017, vol. 153, p. 252

Lamers H. J. G. L. M., Zickgraf F.-J., de Winter D., Houziaux L., Zorec J., An improved classification of B[e]-type stars, A\&A, 1998, vol. 340, p. 117

Ledrew G., The Real Starry Sky, JRASC, 2001, vol. 95, p. 32

Lee U., Osaki Y., Saio H., Viscous excretion discs around Be stars, MNRAS, 1991, vol. 250, p. 432 
Lomb N. R., Least-squares frequency analysis of unequally spaced data, Ap\&SS, 1976, vol. 39 , p. 447

Maciel W.-J., Introdução à Estrutura e Evolução Estelar. EDUSP, 1999

Martayan C., Floquet M., Hubert A. M., Gutiérrez-Soto J., Fabregat J., Neiner C., Mekkas M., Be stars and binaries in the field of the SMC open cluster NGC 330 with VLTFLAMES, A\&A, 2007, vol. 472, p. 577

Martayan C., Frémat Y., Hubert A.-M., Floquet M., Zorec J., Neiner C., Effects of metallicity, star-formation conditions, and evolution in B and Be stars. II. Small Magellanic Cloud, field of NGC330, A\&A, 2007, vol. 462, p. 683

Mason B. D., Hartkopf W. I., Gies D. R., Henry T. J., Helsel J. W., The High Angular Resolution Multiplicity of Massive Stars, AJ, 2009, vol. 137, p. 3358

Meilland A., Millour F., Kanaan S., Stee P., Petrov R., Hofmann K.-H., Natta A., Perraut K., First spectro-interferometric survey of Be stars. I. Observations and constraints on the disk geometry and kinematics, A\&A, 2012, vol. 538, p. A110

Mennickent R. E., Pietrzyński G., Gieren W., Szewczyk O., On Be star candidates and possible blue pre-main sequence objects in the Small Magellanic Cloud, A\&A, 2002, vol. 393 , p. 887

Mennickent R. E., Vogt N., Sterken C., Long-term photometry of Be stars. I. Fading events and variations on time scales of years., A\&AS, 1994, vol. 108, p. 237

Monnier J. D., Zhao M., Pedretti E., Thureau N., Ireland M., Muirhead P., Berger J.-P., Millan-Gabet R., Van Belle G., ten Brummelaar T., McAlister H., Ridgway S., Turner N., Sturmann L., Sturmann J., Berger D., Imaging the Surface of Altair, Science, 2007, vol. 317 , p. 342

Morel T., Castro N., Fossati L., Hubrig S., Langer N., Przybilla N., Schöller M., Carroll e. a., The B Fields in OB Stars (BOB) Survey. In New Windows on Massive Stars , vol. 307 of IAU Symposium, 2015, p. 342

Okazaki A. T., Long-term V/R variations of Be stars due to global one-armed oscillations of equatorial disks, PASJ, 1991, vol. 43, p. 75 
Okazaki A. T., Viscous Transonic Decretion in Disks of Be Stars, PASJ, 2001, vol. 53, p. 119

Okazaki A. T., Theory vs. Observation of Circumstellar Disks and Their Formation. In Active OB-Stars: Laboratories for Stellare and Circumstellar Physics, vol. 361 of Astronomical Society of the Pacific Conference Series, 2007, p. 230

Okazaki A. T., Bate M. R., Ogilvie G. I., Pringle J. E., Viscous effects on the interaction between the coplanar decretion disc and the neutron star in Be/X-ray binaries, MNRAS, 2002, vol. 337, p. 967

Oudmaijer R. D., Wheelwright H. E., Carciofi A. C., Bjorkman J. E., Bjorkman K. S., Spectrally and spatially resolved $\mathrm{H} \alpha$ emission from Be stars: their disks rotate Keplerian. In Active OB Stars: Structure, Evolution, Mass Loss, and Critical Limits, vol. 272 of IAU Symposium, 2011, p. 418

Papaloizou J. C. B., Lin D. N. C., Theory Of Accretion Disks I: Angular Momentum Transport Processes, ARA\&A, 1995, vol. 33, p. 505

Paul K. T., Subramaniam A., Mathew B., Mennickent R. E., Sabogal B., Study of candidate Be stars in the Magellanic Clouds using near-infrared photometry and optical spectroscopy, MNRAS, 2012, vol. 421, p. 3622

Pietrzyński G., Graczyk D., Gieren W., Thompson I. B., Pilecki B., Udalski A., Soszyński I. e. a., An eclipsing-binary distance to the Large Magellanic Cloud accurate to two per cent, Nature, 2013, vol. 495, p. 76

Porter J. M., On outflowing viscous disc models for Be stars, A\&A, 1999, vol. 348, p. 512

Porter J. M., Rivinius T., Classical Be Stars, PASP, 2003, vol. 115, p. 1153

Pringle J. E., Accretion discs in astrophysics, ARA\&A, 1981, vol. 19, p. 137

Quirrenbach A., Bjorkman K. S., Bjorkman J. E., Hummel C. A., Buscher D. F., Armstrong J. T., Mozurkewich D., Elias II N. M., Babler B. L., Constraints on the Geometry of Circumstellar Envelopes: Optical Interferometric and Spectropolarimetric Observations of Seven Be Stars, ApJ, 1997, vol. 479, p. 477 
Rímulo L. R., The life cycles of viscous decretion disks around Be stars: fundamental disk parameters in the SMC, Iag-Usp Oca-Uns, 2017, Tese de Doutorado

Rímulo L. R., Carciofi A. C., Vieira R. G., Rivinius T., Faes D. M., Figueiredo A. L., Bjorkman J. E., Georgy C., Ghoreyshi M. R., Soszyński I., The life cycles of Be viscous decretion discs: fundamental disc parameters of 54 SMC Be stars, MNRAS, 2018, vol. 476 , p. 3555

Rivinius T., Baade D., Štefl S., Non-radially pulsating Be stars, A\&A, 2003, vol. 411, p. 229

Rivinius T., Carciofi A. C., Martayan C., Classical Be stars. Rapidly rotating B stars with viscous Keplerian decretion disks, A\&A Rev., 2013, vol. 21, p. 69

Rivinius T., Štefl S., Baade D., Bright Be-shell stars, A\&A, 2006, vol. 459, p. 137

Sabogal B. E., Mennickent R. E., Pietrzyński G., Gieren W., Be star candidates in the Large Magellanic Cloud: the catalogue and comparison with the Small Magellanic Cloud sample, MNRAS, 2005, vol. 361, p. 1055

Saio H., Cameron C., Kuschnig R., Walker G. A. H., Matthews J. M., Rowe J. F., Lee U., Huber D., Weiss W. W., Guenther D. B., Moffat A. F. J., Rucinski S. M., Sasselov D., g-modes in the late-type Be star $\beta$ CMi detected by the MOST satellite^1, Communications in Asteroseismology, 2007, vol. 150, p. 215

Salpeter E. E., The Luminosity Function and Stellar Evolution., ApJ, 1955, vol. 121, p. 161

Sana H., Evans C. J., The multiplicity of massive stars. In Active OB Stars: Structure, Evolution, Mass Loss, and Critical Limits , vol. 272 of IAU Symposium, 2011, p. 474

Scargle J. D., Studies in astronomical time series analysis. II - Statistical aspects of spectral analysis of unevenly spaced data, ApJ, 1982, vol. 263, p. 835

Secchi A., Schreiben des Herrn Prof. Secchi, Directors der Sternwarte des Collegio Romano, an den Herausgeber, Astronomische Nachrichten, 1866, vol. 68, p. 63

Shakura N. I., Sunyaev R. A., Black holes in binary systems. Observational appearance., A\&A, 1973, vol. 24, p. 337 
Sigut T. A. A., Patel P., The Correlation between $\mathrm{H} \alpha$ Emission and Visual Magnitude during Long-term Variations in Classical Be Stars, ApJ, 2013, vol. 765, p. 41

Sterken C., Vogt N., Mennickent R. E., Long-term photometry of Be stars. II. Periodic variations on time scales of days to months., A\&A, 1996, vol. 311, p. 579

Struve O., On the Origin of Bright Lines in Spectra of Stars of Class B, ApJ, 1931, vol. 73, p. 94

Townsend R. H. D., Owocki S. P., Howarth I. D., Be-star rotation: how close to critical?, MNRAS, 2004, vol. 350, p. 189

Udalski A., The Optical Gravitational Lensing Experiment. Real Time Data Analysis Systems in the OGLE-III Survey, Acta Astron., 2003, vol. 53, p. 291

Udalski A., Kubiak M., Szymanski M., Optical Gravitational Lensing Experiment. OGLE2 - the Second Phase of the OGLE Project, Acta Astron., 1997, vol. 47, p. 319

Udalski A., Szymanski M., Kaluzny J., Kubiak M., Mateo M., The Optical Gravitational Lensing Experiment, Acta Astron., 1992, vol. 42, p. 253

Udalski A., Szymanski M. K., Soszynski I., Poleski R., The Optical Gravitational Lensing Experiment. Final Reductions of the OGLE-III Data, Acta Astron., 2008, vol. 58, p. 69

Udalski A., Szymański M. K., Szymański G., OGLE-IV: Fourth Phase of the Optical Gravitational Lensing Experiment, Acta Astron., 2015, vol. 65, p. 1

Štefl S., Carciofi A. C., Baade D., Rivinius T., Otero S., Le Bouquin J.B., Fabregat J., Okazaki A. T., Rantakyrö F., The 2008+ outburst of the Be star $28 \mathrm{CMa}$ - a multi-instrument study†.InActiveOBStars : Structure, Evolution, MassLoss, andCriticalLimits, vol. 272of I AU Symposium, 2011, p. 430

Vieira R. G., Carciofi A. C., Bjorkman J. E., The pseudo-photosphere model for the continuum emission of gaseous discs, MNRAS, 2015, vol. 454, p. 2107

Vieira R. G., Carciofi A. C., Bjorkman J. E., Rivinius T., Baade D., Rímulo L. R., The life cycles of Be viscous decretion discs: time-dependent modelling of infrared continuum observations, MNRAS, 2017, vol. 464, p. 3071 
von Zeipel H., The radiative equilibrium of a rotating system of gaseous masses, MNRAS, 1924, vol. 84, p. 665

Wade G. A., Grunhut J. H., MiMeS Collaboration The MiMeS Survey of Magnetism in Massive Stars. In Circumstellar Dynamics at High Resolution , vol. 464 of Astronomical Society of the Pacific Conference Series, 2012, p. 405

Wade G. A., Neiner C., Alecian E., Grunhut J. H., Petit V., Batz B. d., Bohlender D. A., Cohen D. H., Henrichs e. a., The MiMeS survey of magnetism in massive stars: introduction and overview, MNRAS, 2016, vol. 456, p. 2

Walker G. A. H., Kuschnig R., Matthews J. M., Reegen P., Kallinger T., Kambe E., Saio H., Harmanec P., Guenther D. B., Moffat A. F. J., Rucinski S. M., Sasselov D., Weiss W. W., et al. Pulsations of the Oe Star $\zeta$ Ophiuchi from MOST Satellite Photometry and Ground-based Spectroscopy, ApJ, 2005, vol. 623, p. L145

Wheelwright H. E., Bjorkman J. E., Oudmaijer R. D., Carciofi A. C., Spectroastrometry and NLTE Modeling of the Disk Around the Be Star $\beta$ CMi. In Circumstellar Dynamics at High Resolution, vol. 464 of Astronomical Society of the Pacific Conference Series, 2012, p. 205

Wheelwright H. E., Bjorkman J. E., Oudmaijer R. D., Carciofi A. C., Bjorkman K. S., Porter J. M., Probing the properties of Be star discs with spectroastrometry and NLTE radiative transfer modelling: $\beta$ CMi, MNRAS, 2012, vol. 423, p. L11

Wood K., Bjorkman J. E., Whitney B. A., Code A. D., The Effect of Multiple Scattering on the Polarization from Axisymmetric Circumstellar Envelopes. I. Pure Thomson Scattering Envelopes, ApJ, 1996, vol. 461, p. 828

Wood K., Bjorkman K. S., Bjorkman J. E., Deriving the Geometry of Be Star Circumstellar Envelopes from Continuum Spectropolarimetry. I. The Case of $\zeta$ Tauri, ApJ, 1997, vol. 477 , p. 926 